\title{
Zur Strahlungshydrodynamik der Akkretionssäulen auf magnetischen weißen Zwergen
}

\author{
Dissertation \\ zur Erlangung des Doktorgrades \\ der Mathematisch-Naturwissenschaftlichen Fakultäten \\ der Georg-August Universität zu Göttingen
}

vorgelegt von

Andreas Fischer

aus Northeim

Göttingen 1999 
D 7

Referent: Prof. Dr. K. Beuermann

Korreferent: Prof. Dr. H. Eckelmann

Tag der mündlichen Prüfung: 27. Januar 2000 


\section{Inhaltsverzeichnis}

1 Einleitung 1

1.1 Kataklysmische Veränderliche . . . . . . . . . . . . . . . 1

$\square 2$ Polare . . . . . . . . . . . . . . . . . . . . . . . 4

1.2 .1 Definierende Eigenschaften . . . . . . . . . . . . . 4

1.2 .2 Die Emissionsregion $\ldots \ldots \ldots \ldots \ldots$

1.2 .3 Zustände hoher Akkretionsrate $\ldots \ldots \ldots \ldots \ldots$

1.2 .4 Zustände niedriger Akkretionsrate . . . . . . . . . . . . . . . . 13

1.2 .5 Überschuß an weicher Röntgenstrahlung . . . . . . . . . . . . . 16

1.3 Zielsetzung und Aufbau der Arbeit . . . . . . . . . . . . . . . . . . . . 19

2 Hydrodynamisches Modell des Akkretionsstroms 21

2.1 Stationäres Ein-Teilchen-Plasma mit Bremsstrahlungsverlusten . . . . . . . 21

2.2 Stationäres Ein-Teilchen-Plasma mit Brems- und Zyklotronstrahlungsver-

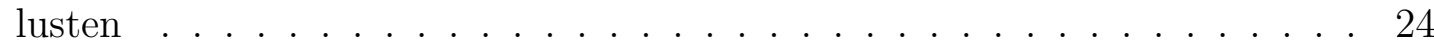

2.3 Instationäres Zwei-Teilchen-Plasma $\ldots \ldots \ldots \ldots . \ldots \ldots$

3 Stationäre Strahlungshydrodynamik 33

3.1 Grundgleichungen . . . . . . . . . . . . . . . . . . . . . . . 33

3.2 Annahmen und Näherungen $\ldots \ldots \ldots \ldots \ldots$

3.2 .1 Stationarität und eindimensionale Strömung . . . . . . . . . . . 35

32.2 ldeales Fluid . . . . . . . . . . . . . . . . 36

3.2 .3 Einfluß der Wärmeleitung . . . . . . . . . . . . . . . . . 40

$3.2 .4 \quad$ Behandlung der Streuung im Strahlungstransport . . . . . . . . . . 41

3.3 Betrachtetes Gleichungssystem und Lösungsmethode . . . . . . . . . . . . 44

3.4 Ergebnisse . . . . . . . . . . . . . . . . . . . . . . . . . . . . 47

3.4 .1 Schichtung der Emissionsregion $\ldots \ldots \ldots \ldots \ldots$

3.4 .2 Spektrum der Emissionsregion . . . . . . . . . . . . . . . 51

$3.5 \quad$ Vergleich mit Ein-Teilchen-Modellen $\ldots \ldots \ldots \ldots \ldots$

3.5.1 Ein-Teilchen-Plasma mit Bremsstrahlungsverlusten . . . . . . . . 58

3.5.2 Ein-Teilchen-Plasma mit Brems- und Zyklotronstrahlungsverlusten 61

3.5 .3 Bewertung . . . . . . . . . . . . . . . . . 68

3.6 Auswirkungen der genäherten Geometrie $\ldots \ldots \ldots$. . . . . . . . . . 69

3.6 .1 Schichtungen großser Höhe $\ldots \ldots \ldots \ldots$ 
3.6.2 Seitlich begrenzte Akkretionsregion . . . . . . . . . . . . . . . . . . 72

4 Anwendung der Ergebnisse auf den Polar AM Herculis 79

4.1 Modellierung der optischen Lichtkurve . . . . . . . . . . . . . . . . . . . . 79

4.2 Spektrale Energieverteilung . . . . . . . . . . . . . . . . . . . . . . . . . . 84

$4.2 .1 \quad$ „High-state" Zustand des Systems . . . . . . . . . . . . . . . . . . 84

$4.2 .2 \quad$ „Low-state" Zustand des Systems . . . . . . . . . . . . . . . . . . . 95

4.2 .3 Diskussion . . . . . . . . . . . . . . . . . . . . . 105

$\begin{array}{lll}5 & \text { Zusammenfassung und Ausblick } & 109\end{array}$

$\begin{array}{ll}\text { Literaturverzeichnis } & 113\end{array}$

$\begin{array}{lr}\text { Danksagung } & 119\end{array}$

\begin{tabular}{lr}
\hline Veröffentlichungen & 121
\end{tabular}

$\begin{array}{lc}\text { Lebenslaut } & 123\end{array}$ 


\section{Kapitel 1}

\section{Einleitung}

\subsection{Kataklysmische Veränderliche}

Die Geometrie eines Doppelsternsystems wird durch das Massenverhältnis der beiden Sterne und durch die Orbitalperiode bestimmt. Sind die beiden Komponenten in synchroner Rotation mit der Orbitalbewegung, dann können die im System wirkenden konservativen Kräfte durch den Verlauf des „Roche-Potentials“, das sich in einem mitrotierenden Bezugssystem aus einem Term für die Gravitationskraft und einem für die Zentrifugalkraft zusammensetzt, veranschaulicht werden. In Abbildung 1.1 (folgende Seite) wird dieses Potential durch Äquipotentiallinien in der Bahnebene, für ein Massenverhältnis $M_{1} / M_{2}=5$ und eine Orbitalperiode $P=3,5 \mathrm{~h}$ (d.h. Abstand der Komponenten $a=8,6 \cdot 10^{10} \mathrm{~cm}$ ), dargestellt. Der dadurch festgelegte Massenschwerpunkt ist mit „CM“ (engl. „Center of Mass") bezeichnet; durch ihn verläuft auch die Drehachse des Systems. Die Orte der Sternmittelpunkte sind durch $M_{1}$ bzw. $M_{2}$ gekennzeichnet und werden von konzentrischen Kreisen des Gravitationspotentials umgeben, wobei die Komponente $M_{1}$ mit der größeren Masse einen „tieferen" Potentialtopf erzeugt. Erst in einiger Entfernung von den Sternen wird die Zentrifugalkraft bedeutsam und überlagert sich der Gravitationskraft; die Äquipotentiallinien weichen hier von der kreisförmigen Gestalt des Gravitationspotentials erheblich ab. Die Punkte $L_{1}, \ldots, L_{5}$ kennzeichnen die Librationspunkte („Lagrange-Punkte“), in denen sich Gravitation und Fliehkraft ausgleichen. Dabei sind $L_{1}, \ldots, L_{3}$ bezüglich der Bewegung von Probeteilchen labile und $L_{4}, L_{5}$ stabile Bereiche des Potentialverlaufs. ( $L_{4}$ und $L_{5}$ sind lokale Maxima, die Bewegung eines Probeteilchens in ihrer Umgebung wird aber durch die nicht im Roche-Potential erfaßte Coriolis-Kraft stabilisiert.)

Um jeden der beiden Sterne gibt es eine größte geschlossene Äquipotentialfläche, die die Masse der Komponente beeinhaltet. Das darin eingeschlossene Volumen nennt man "Roche-Volumen" des Sterns; es ist ungefähr rotationssymmetrisch um die Systemachse $\overline{M_{1} M_{2}}$. Der Punkt $L_{1}$ (,,innerer Lagrange-Punkt“) ist ein Sattelpunkt des Potentialverlaufs und verbindet die beiden Roche-Volumina. Materie außerhalb dieser Volumina kann man nicht eindeutig einem der Sterne zuordnen. 


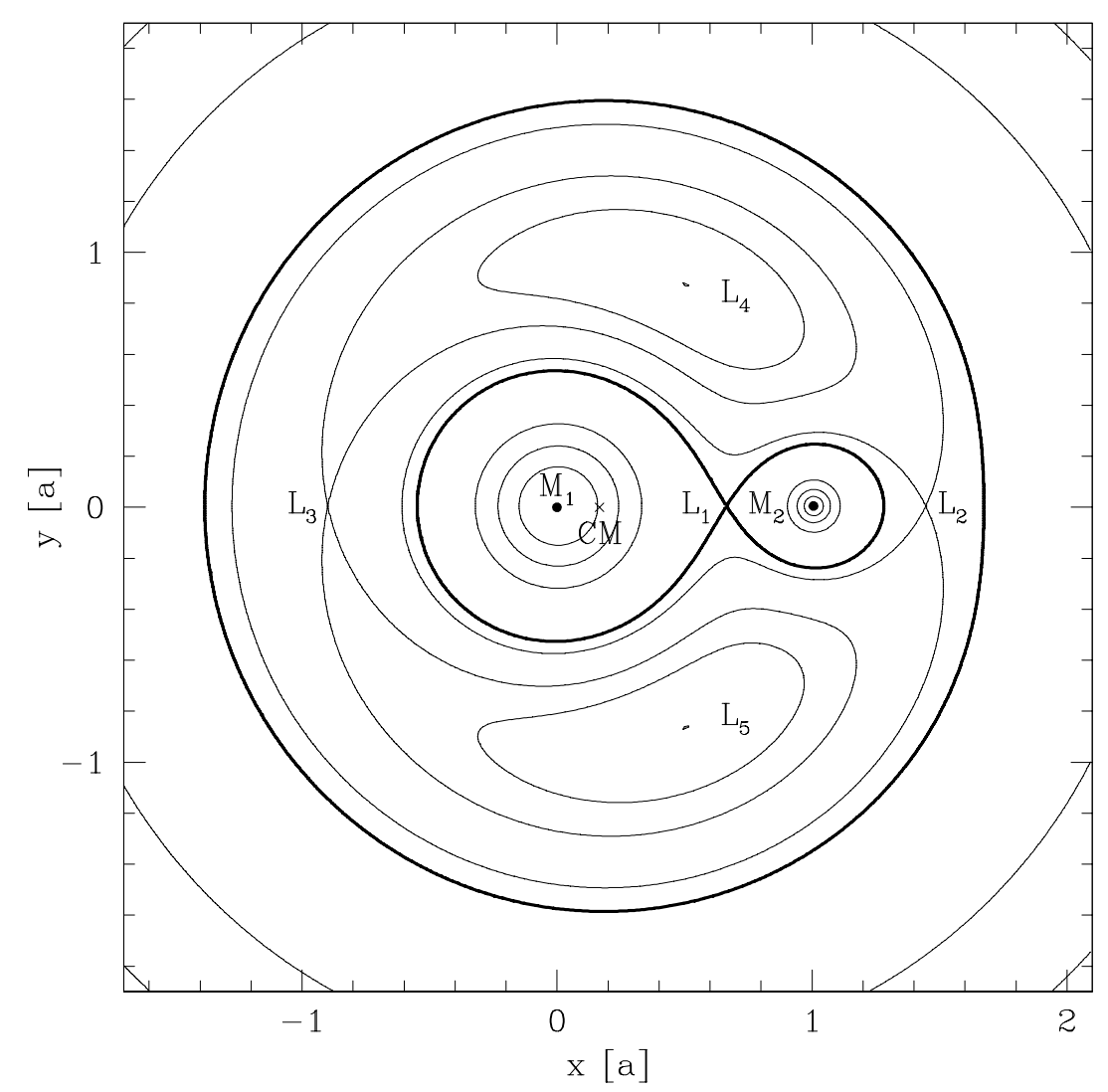

Abbildung 1.1: Roche-Geometrie eines Doppelsterns. Dargestellt sind die Äquipotentiallinien des Roche-Potentials in der Bahnebene, für ein Massenverhältnis $M_{1} / M_{2}=5$. Die Achsen sind in Einheiten des Abstands $a=8,6 \cdot 10^{10} \mathrm{~cm}$ der Komponenten skaliert. $L_{1}, \ldots, L_{5}$ sind die Lagrange-Punkte, CM ist der Massenschwerpunkt des Systems. Das sog. „Roche-Volumen“ wird durch Drehsymmetrie der stark ausgezogenen Linie durch $L_{1}$ um die Achse $\overline{M_{1} M_{2}}$ annähernd beschrieben; $L_{4}$ und $L_{5}$ sind lokale Maxima des Potentials. Die beiden stark ausgezogenen Linien weisen den selben Potentialwert auf. (Ich danke Dr. B.T. Gänsicke für diese Abb.)

Ergebnisse aus Sternentwicklungsrechnungen zeigen, daß Hauptreihensterne mit hoher Masse sich schneller zu Riesensternen entwickeln, als solche mit geringer Masse (Kippenhahn \& Weigert 1990). Besteht das Doppelsternsystem aus zwei Hauptreihensternen, so wird sich im Laufe der Entwicklung die massereiche Komponente $M_{1}$ stärker ausdehnen als die Komponente $M_{2}$ und eher ihr Roche-Volumen ausfüllen; der Doppelstern ist dann zu einem „halbgetrennten System“ geworden.

Bei einer weiteren Expansion wird Materie über den $L_{1}$-Punkt in den Einzugsbereich des Gravitationspotentials der Komponente $M_{2}$ gebracht und dort abgelagert. Solch eine Materieaufnahme durch ein Objekt nennt man „Akkretion“. Da beim Ablagern auf die masseärmere Komponente Materie vom Schwerpunkt weg bewegt wird, reagiert das System bei Drehimpulserhaltung mit einer Verringerung des Abstandes $a$. Damit verkleinert sich aber auch das Roche-Volumen und der Materiestrom von $M_{1}$ wird verstärkt, 
denn die Ausdehnung dieser Komponente kann nicht im selben Maß abnehmen wie das Roche-Volumen. Dieser starke Überstrom kann von der Komponente $M_{2}$ allein nicht aufgenommen werden. Stattdessen wird das Potentialfeld soweit aufgefüllt, bis beide Komponenten von Materie eingehüllt sind (engl. „common envelope“). In dieser Hülle wird durch Reibung ein Teil des Drehimpulses in Wärme umgewandelt, und die so aufgeheizte Materie kann entlang der Bahnebene aus dem System befördert werden. Da hierbei ebenso Drehimpuls verlorengeht, nimmt der Abstand der Komponenten weiter ab und der Materieverlust erhöht sich. Numerische Simulationen dieser Prozesse zeigen, daß die gemeinsame Hülle abgestoßen werden kann, bevor die beiden Komponenten miteinander verschmelzen (siehe z. B. den Übersichtsartikel von Livio 1994).

Als Endstadium des Szenariums bleibt ein getrenntes System übrig, in dem keine der beiden Komponenten ihr Roche-Volumen ausfüllt, also auch keine Akkretion über den $L_{1}$-Punkt mehr stattfindet. Je nach Anfangsmasse von $M_{1}$ entsteht ein weißer Zwerg, ein Neutronenstern oder ein schwarzes Loch, während $M_{2}$ vermutlich nahezu unverändert bleibt und weiterhin ein unvollständig entwickelter, massearmer Hauptreihenstern ist.

Der Doppelstern wird wieder zu einem akkretierenden System, wenn sich die massearme Komponente $M_{2}$ zu einem Riesen entwickelt. Zwar gewinnt $M_{2}$ durch die Akkretion an Masse, dennoch ist der typischerweise resultierende Wert zu klein, als daß die Entwicklungszeit wesentlich geringer als das Alter des Universums wird. Dieser Fall scheidet daher als Erklärung der beobachteten akkretierenden Doppelsterne i. allg. aus.

Verliert das getrennte System an Drehimpuls, wird der Abstand der Sterne (und damit das Roche-Volumen) weiter verringert und Akkretion kann erneut einsetzen. Ein möglicher Mechanismus für Drehimpulsverlust ist der, von der allgemeinen Relativitätstheorie vorhergesagte, Energieverlust durch Gravitationsstrahlung, die von zwei sich umkreisenden Massen abgegeben wird und ein Drehmoment auf das System ausübt. Dieser Verlust ist effektiv, wenn das System eine kleine Periode aufweist oder die Massen der Komponenten groß sind (siehe z. B. Landau \& Lifshitz 1962). Eine andere Möglichkeit, die offenbar bei großen Perioden dominiert, wird mit dem englischen Begriff "magnetic braking“ bezeichnet. Vom Hauptreihenstern $M_{2}$ wird ein Strom von ionisierten Teilchen abgegeben („Sternenwind“), die, bei vorhandenem Magnetfeld, entlang der Feldlinien transportiert werden und bis zum Alfvén-Punkt (in dem Gleichheit von Plasma- und Magnetfelddruck herrscht) starr an der Rotation des Sterns teilnehmen. Dabei gewinnen sie an Drehimpuls, der aus der Rotation des Sterns entnommen wird. Erst hinter dem Alfvén-Punkt können die Teilchen frei im Gravitationsfeld abströmen und so Drehimpuls aus dem System entfernen. Da sich die Komponente $M_{2}$ durch den Einfluß von Gezeitenkräften stets in synchroner Rotation mit der Orbitalbewegung befindet, greift ein Drehmoment effektiv am System an und bremst es ab (Eggleton 1976); die Roche-Volumina verkleinern sich. Nach ausreichendem Drehimpulsverlust füllt $M_{2}$ sein Roche-Volumen aus, und die kompakte Komponente $M_{1}$ akkretiert nun Materie über den $L_{1}$-Punkt.

Wenn der akkretierende Stern (im folgenden auch „Primärkomponente“ genannt) ein weißer Zwerg ist, bezeichnet man das System als kataklysmischen Veränderlichen (engl. „cataclysmic variable“, CV). In solchen Systemen ist der massegebende Partner („Sekundärkomponente") häufig ein massearmer Zwergstern vom Spektraltyp K oder M mit 
Masse $M=0,1 \ldots 0,8 M_{\odot}\left(M_{\odot}:\right.$ Sonnenmasse $)$. Da die Gesamtmasse der Sterne nicht viel größer als $2 M_{\odot}$ sein kann und die Orbitalperiode typischerweise bei einigen Stunden liegt, ist der Abstand der Komponenten von der Größenordnung des Sonnenradius.

Die akkretierte Materie setzt die beim Fall im Gravitationspotential des weißen Zwergs gewonnene Energie in Form von Strahlung frei, deren Leuchtkraft gegeben ist durch:

$$
L_{\mathrm{acc}}=\frac{G M_{\mathrm{wd}} \dot{M}}{R_{\mathrm{wd}}} .
$$

Darin ist $G$ die Gravitationskonstante, $M_{\text {wd }}$ und $R_{\text {wd }}$ Masse bzw. Radius des weiBen Zwergs und $\dot{M}$ die Akkretionsrate (in $\mathrm{g} \mathrm{s}^{-1}$ ). Bei typischen Akkretionsraten von $\dot{M}=10^{15} \ldots 10^{17} \mathrm{~g} \mathrm{~s}^{-1}=10^{-11} \ldots 10^{-9} M_{\odot} \mathrm{a}^{-1}$ erhält man Leuchtkräfte von $L_{\text {acc }}=$ $10^{31} \ldots 10^{34} \mathrm{erg} \mathrm{s}^{-1}$, d.h. $3 \cdot 10^{-3} \ldots 3 L_{\odot}\left(L_{\odot}\right.$ : Sonnenleuchtkraft $)$. Diese Energien werden in einer relativ kleinen Region freigesetzt und erzeugen dort Temperaturen in der Größenordnung $10^{8} \mathrm{~K}$. CVs sind daher helle UV- und Röntgenquellen, und die akkretierte Materie weist im allgemeinen einen hohen Ionisationsgrad auf.

\subsection{Polare}

\subsubsection{Definierende Eigenschaften}

Da kataklysmische Variable in Abhängigkeit von ihren Parametern wie z. B. Massenverhältnis, Orbitalperiode oder Akkretionsrate stark unterschiedliche Eigenschaften in der beobachteten Strahlung zeigen, gibt es mehrere Klassifikationen, um individuelle Systeme einzuordnen. (Eine umfassende Darstellung der CVs findet man in Warner 1995.) Die Zuordnung muß dabei nicht immer eindeutig sein, so daß ein System mehreren Klassen angehören kann. Eine mögliche Unterteilung stützt sich auf das Magnetfeld des weißen Zwergs.

Der Akkretionsstrom vom Sekundärstern wird beim Fall im Gravitationspotential der Primärkomponente durch die Coriolis-Kraft aus seiner Bewegungsrichtung abgelenkt. Dabei bewirkt die Viskosität des Strommaterials, daß sich die Materie in Form einer äquatorialen Akkretionsscheibe um den weißen Zwerg ansammelt und einen Teil ihres Drehimpulses verliert. Über eine Grenzschicht im Bereich der Sternoberfläche wird das Material schließlich auf den weißen Zwerg gebracht. Die Strahlung dieser Systeme im optischen Spektralbereich und im UV wird im wesentlichen durch die Scheibe bestimmt, während die Grenzschicht im Röntgenbereich zu sehen ist.

Da der weiße Zwerg im allgemeinen ein Magnetfeld besitz, gibt es ein Gebiet der Akkretionsscheibe, in dem der magnetische Druck den Staudruck übersteigt und das Plasma an das Magnetfeld ankoppelt:

$$
\frac{B^{2}}{8 \pi} \geq \rho v^{2}=\frac{\dot{M}}{A} v,
$$

mit $B$ der Magnetfeldstärke, $\rho$ der Massendichte, $A$ dem Stromquerschnitt am $L_{1}$-Punkt und $v$ der Geschwindigkeit der Teilchen. An diesem Ort wird die Materie aus der Scheibe 


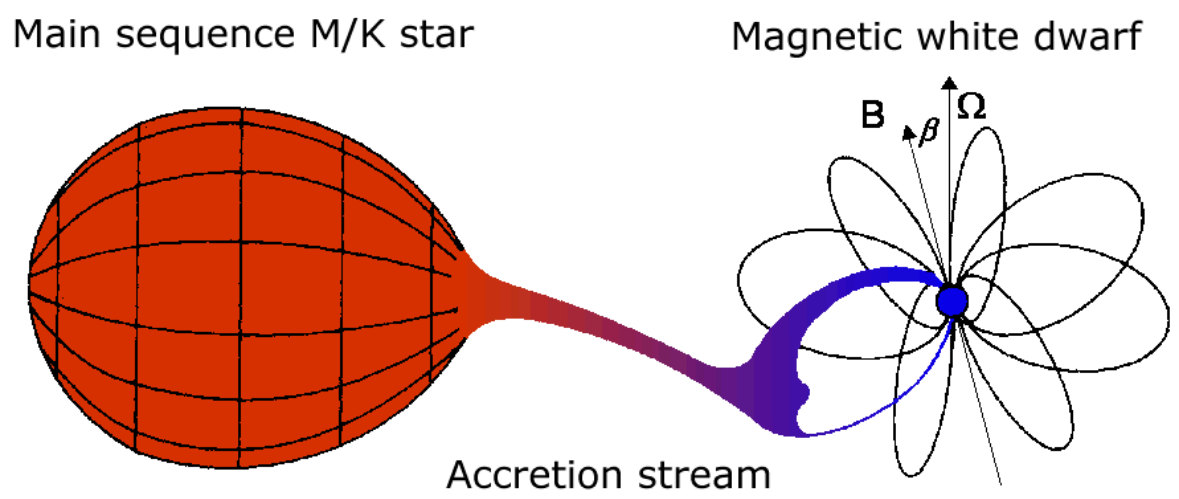

Abbildung 1.2: Akkretion in magnetischen kataklysmischen Veränderlichen (Abbildung nach Cropper 1990). Der Akkretionsstrom wird entlang der Magnetfeldlinien auf den weißen Zwerg geführt. (Ich danke Dr. B.T. Gänsicke für diese Abb.)

hinaus und entlang der Magnetfeldlinien in die Nähe der Polkappen des weißen Zwergs gebracht. Bei geringer Polfeldstärke liegt dieser Bereich in der Nähe des Sterns und erzeugt nur eine kleine Region, in der die Scheibe aufgebrochen ist, während sie in einer größeren Entfernung ungestört bleibt. Da die Bestimmung der Magnetfeldstärke in solchen Systemen schwierig ist, läßt sich ein Grenzwert für das Aufbrechen nur abschätzen; er liegt ungefähr bei $B=5 \mathrm{MG}$.

Wird das Magnetfeld so groß, daß es die überströmende Materie wesentlich beeinflußt, liegt der Ankopplungsbereich nahe beim $L_{1}$-Punkt, und keinerlei Akkretionsscheibe bildet sich aus (Abb. 1.2). In solchen Systemen bewirkt das Magnetfeld weiterhin eine synchrone Rotation der beiden Komponenten, so daß sich immer die selben Flächen gegenüberstehen. Dadurch ist der Auftreffpunkt am weißen Zwerg - und damit die gesamte Akkretionsgeometrie - quasi-stationär. Die für diesen Typ von CVs charakteristische Röntgenlichtkurve kann daher in erster Näherung durch die Ansicht des Akkretionsflecks zu verschiedenen Phasen des Orbits erklärt werden: Phasen mit hoher beobachteter Intensität (engl. „bright-phase“) entsprechen paralleler Sicht zur Flächennormalen des Flecks, während eine niedrige Intensität (engl. „faint-phase") einen großen Sichtwinkel nahelegt.

Das kennzeichnende Element magnetischer CVs ist vor allem ihre hohe, phasenabhängige lineare und zirkulare Polarisation, die durch Zyklotronstrahlung der um die Feldlinien gyrierenden Elektronen erzeugt wird (Bekefi 1966) und die Lichtkurven im Infraroten und Optischen bestimmt. Da die Intensität der Zyklotronstrahlung am größten ist, wenn der Sichtwinkel zum Magnetfeld (als parallel zur Flecknormalen angenommen) groß ist, ist die entsprechende Lichtkurve ungefähr gegenphasig zur Röntgenlichtkurve.

Der starken Modulation ihrer Strahlung wegen, nennt man die magnetischen kataklysmischen Veränderlichen - in Anlehnung an die (Radio-)Pulsare - auch „Polare“. Ein weiterer synonym benutzer Name ist „AM Her-System“, denn der erste identifizierte CV dieser Art war der variable Stern AM im Sternbild Herkules. (Eine ausführliche Beschreibung der Polare bietet der Übersichtsartikel von Cropper 1990.) Da in der vorliegenden Arbeit nur auf magnetische kataklysmische Variable eingegangen wird, sollen im folgenden Aspekte der Akkretion in diesen Systemen angesprochen werden. 


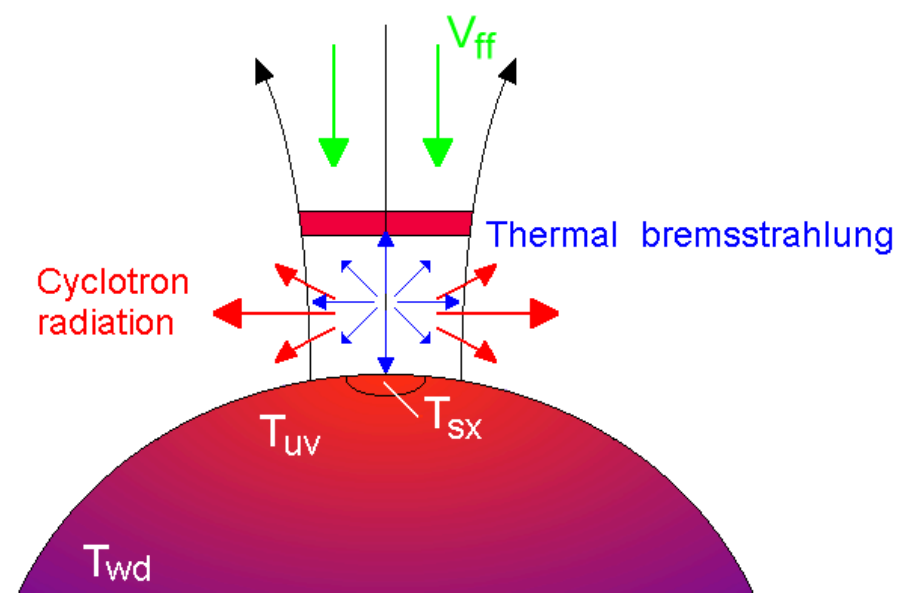

Abbildung 1.3: Vereinfachte Darstellung der Akkretionsregion in Polaren (aus Gänsicke 1997). Die Materie wird entlang der Magnetfeldlinien geführt, abgebremst und durch Strahlung gekühlt, bis die Sternoberfläche erreicht ist.

\subsubsection{Die Emissionsregion}

Die Akkretionsregion auf der Oberfläche des weißen Zwergs ist der Entstehungsort der für Polare charakteristischen Strahlung (Abb. 1.3): Zyklotronstrahlung, deren Spektralbereich je nach Magnetfeldstärke im Infraroten bis Ultravioletten liegt, thermische Bremsstrahlung im harten Röntgenbereich und weiche Röntgenstrahlung, die einen schwarzkörperartigen Verlauf zeigt. Hinzu kommen noch die schwachen Komponenten des Akkretionsstroms und des Sekundärsterns, sowie die Emission des weißen Zwergs. Die zutreffende Beschreibung der Prozesse in der Emissionsregion ist daher notwendig, um Beobachtungsdaten richtig zu interpretieren. Andererseits geben neue Beobachtungen auch immer Impulse zur Weiterentwicklung der theoretischen Modelle. Die Vielzahl der auftretenden Phänomene macht es jedoch schwierig eine einheitliche Theorie aufzustellen, die alle Beobachtungen erklären kann. So gibt es in Polaren lang andauernde Zustände mit sehr niedriger bzw. sehr hoher Akkretionsrate (engl. „low-states“ bzw. „high-states“), in denen verschiedene physikalische Prozesse dominieren.

In der Beschreibung der Akkretionsvorgänge ist grundlegende Tatsache, daß der im Gravitationspotential freifallende Akkretionsstrom abgebremst werden muß, um die Materie auf der Sternoberfläche abzulagern. Mit der Frei-Fall-Geschwindigkeit $v_{\mathrm{ff}}$ in der Nähe des Sterns $\left(R_{\odot}:\right.$ Sonnenradius)

$$
v_{\mathrm{ff}}\left(R_{\mathrm{wd}}\right)=\sqrt{\frac{2 G M_{\mathrm{wd}}}{R_{\mathrm{wd}}}}=6,2 \cdot 10^{7} \frac{\mathrm{cm}}{\mathrm{s}} \sqrt{\frac{M_{\mathrm{wd}}}{M_{\odot}} \frac{R_{\odot}}{R_{\mathrm{wd}}}}
$$

und der adiabatischen Schallgeschwindigkeit $\left(k_{\mathrm{B}}\right.$ : Boltzmannkonstante, $\gamma$ : Adiabatenindex, $\mu$ : mittlere relative Teilchenmasse, $m_{\mathrm{p}}$ : Protonenmasse, $T$ : Temperatur)

$$
v_{\mathrm{s}}^{\mathrm{ad}}=\sqrt{\gamma \frac{k_{\mathrm{B}} T}{\mu m_{\mathrm{p}}}}=1,6 \cdot 10^{6} \frac{\mathrm{cm}}{\mathrm{s}} \sqrt{\frac{T}{10^{4} \mathrm{~K}}}
$$


für ein vollständig ionisiertes Wasserstoff-Plasma $(\gamma=5 / 3, \mu=1 / 2)$, fällt der Strom bei typischen Temperaturen von $T=10^{4} \mathrm{~K}$ mit Überschallgeschwindigkeit. Wird nun die akkretierte Energie nicht effektiv wieder abgegeben (z. B. durch Strahlung), bildet sich ein starker hydrodynamischer Stoß (Machzahl $M a:=v / v_{\mathrm{s}}>1$ ) aus, in dessen Diskontinuität die gerichtete Fallenergie in thermische Energie umgewandelt wird. Aus Massen-, Energieund Impulserhaltung kann man die Veränderung der physikalischen Größen über den Stoß hinweg herleiten (Landau \& Lifshitz 1963):

$$
\frac{\rho_{0}}{\rho_{\mathrm{sh}}}=\frac{v_{\mathrm{sh}}}{v_{0}}=\frac{\gamma-1}{\gamma+1}=\frac{1}{4},
$$

wenn der Index 0(sh) die Größe vor(nach) dem Stoß bezeichnet. Vor einem starken Stoß ist der thermische Druck $P_{0}$ gegen den Staudruck $\rho_{0} v_{0}^{2}$ vernachlässigbar und der Wert hinter der Stoßfront ist:

$$
P_{\mathrm{sh}}=\frac{2}{\gamma+1} \rho_{0} v_{0}^{2}=\frac{3}{4} \rho_{0} v_{0}^{2} .
$$

Mit der idealen Gasgleichung gilt für die Temperatur nach dem Stoß („Rankine-HugoniotTemperatur" oder „Stoßtemperatur"):

$$
T_{\mathrm{sh}}=\frac{2(\gamma-1)}{(\gamma+1)^{2}} \frac{\mu m_{\mathrm{p}}}{k_{\mathrm{B}}} v_{0}^{2}=\frac{3}{16} \frac{\mu m_{\mathrm{p}}}{k_{\mathrm{B}}} v_{0}^{2} .
$$

In der Nähe der Oberfläche des weißen Zwergs ist $v_{0} \approx v_{\mathrm{ff}}\left(R_{\mathrm{wd}}\right)$, so daß

$$
T_{\mathrm{sh}}=8,7 \cdot 10^{6} \mathrm{~K} \mu \frac{M_{\mathrm{wd}}}{M_{\odot}} \frac{R_{\odot}}{R_{\mathrm{wd}}} .
$$

Der Stoß bringt das Plasma auf Unterschallgeschwindigkeiten, heizt es aber auf sehr hohe Temperaturen auf. Von diesem Wert muß es, z.B. durch Strahlung, heruntergekühlt werden, um auf die Oberflächentemperatur des weißen Zwergs zu gelangen. Es bildet sich also eine Schichtung aus, in der die physikalischen Größen von ihren Stoßwerten auf die Parameter des Sterns gebracht werden müssen.

\subsubsection{Zustände hoher Akkretionsrate}

Im Fall einer hohen Akkretionsrate bei nicht zu großem Magnetfeld kann der Akkretionsstrom als hydrodynamisches Fluid beschrieben werden. Optisch dünne Bremsstrahlung ist dann der dominante Verlustprozeß. Optisch dünn bedeuteut hier, daß die Strahlung die Region ohne Wechselwirkung mit der Materie frei verlassen kann. Die spezifische Bremsstrahlungsemissionsrate (in $\operatorname{erg~s}^{-1} \mathrm{~g}^{-1}$ ) für ein vollständig ionisiertes Plasma der Ionenhäufigkeiten $X_{\mathrm{i}}:=\rho / \rho_{\mathrm{i}}$, Ladungszahlen $Z_{\mathrm{i}}$, relative Massen $\mu_{\mathrm{i}}$ und frequenzgemitteltem Gaunt-Faktor $\bar{g}$ ist (Rybicki \& Lightman 1979):

$$
\varepsilon_{\mathrm{ff}}=5,2 \cdot 10^{20} \mathrm{erg} \mathrm{s}^{-1} \mathrm{~g}^{-2} \mathrm{~cm}^{3} \mathrm{~K}^{-\frac{1}{2}} \sum_{\mathrm{i}} \frac{Z_{\mathrm{i}} X_{\mathrm{i}}}{\mu_{\mathrm{i}}} \sum_{\mathrm{i}} \frac{Z_{\mathrm{i}}^{2} X_{\mathrm{i}}}{\mu_{\mathrm{i}}} \bar{g} \rho T^{\frac{1}{2}} .
$$


Als Kühlzeit des Plasmas definiert man das Verhältnis von spezifischer interner Energie $u$ des idealen, einatomigen Gases zu Energieverlust durch Strahlung:

$$
\begin{aligned}
t_{\mathrm{ff}} & =\frac{u}{\varepsilon_{\mathrm{ff}}}=\frac{3 k_{\mathrm{B}} T}{2 \mu m_{\mathrm{p}} \varepsilon_{\mathrm{ff}}(\rho, T)} \\
& =1,6 \cdot 10^{-2} \mathrm{~s}\left(\frac{T}{T_{\mathrm{sh}}}\right)^{\frac{1}{2}} \frac{\rho_{\mathrm{sh}}}{\rho} \frac{1 \frac{\mathrm{g}}{\mathrm{cm}^{2} \mathrm{~s}}}{\dot{m}} \frac{M_{\mathrm{wd}}}{M_{\odot}} \frac{R_{\odot}}{R_{\mathrm{wd}}},
\end{aligned}
$$

für ein vollionisiertes Wasserstoffplasma mit $\dot{m}:=\dot{M} / A_{\text {acc }}$ der Massenstromdichte (in $\left.\mathrm{g} \mathrm{cm}^{-2} \mathrm{~s}^{-1}\right), A_{\text {acc }}$ der Akkretionsfläche und $\bar{g}=1$. Die Höhe der Stoßfront über der Sternoberfläche ist nun proportional dieser Zeitskala (Aizu 1973), und eine einfache Abschätzung liefert:

$$
h_{\mathrm{sh}} \approx v t_{\mathrm{ff}, \mathrm{sh}} \approx \frac{1}{4} v_{\mathrm{ff}} t_{\mathrm{ff}, \mathrm{sh}}=2,4 \cdot 10^{3} \mathrm{~cm} \frac{100 \frac{\mathrm{g}}{\mathrm{cm}^{2} \mathrm{~s}}}{\dot{m}}\left(\frac{M_{\mathrm{wd}}}{M_{\odot}} \frac{R_{\odot}}{R_{\mathrm{wd}}}\right)^{\frac{3}{2}} .
$$

Für große $\dot{m}$ ist $h_{\mathrm{sh}} \ll R_{\mathrm{wd}}$, und die Emissionsregion ist näherungsweise plan-parallel.

Bei zunehmender Magnetfeldstärke kommt Zyklotronstrahlung als weiterer Verlustprozeß hinzu und trägt zu einer zusätzlichen Verringerung der Höhe bei (Fabian, Pringle \& Rees 1976; Masters et al. 1976). Eine einfache Formel läßt sich hierfür angeben, wenn man die Effizienz der Zyklotronstrahlung im Vergleich zur Bremsstrahlung als Parameter auffaßt (Gl. 2.12).

Für eine korrekte Einbeziehung der Zyklotronstrahlungsverluste in das Gleichungssystem, aus dem sich die Struktur der Emissionsregion ergibt, ist es erforderlich, auch die Wechselwirkung der Strahlung mit der Materie zu berücksichtigen. Denn im Gegensatz zur Bremsstrahlung ist Zyklotronstrahlung nicht für alle Frequenzen optisch dünn, sondern ein wesentlicher Teil der Energie verbleibt in der Schicht und trägt zur Heizung mit bei („optisch dicke Strahlung“). Daher muß zusätzlich zu den hydrodynamischen Gleichungen, die den Geschwindigkeits-, Druck- und Dichteverlauf der Region bestimmen, auch eine Gleichung für den Energietransport durch Zyklotronstrahlung gelöst werden (,Strahlungstransportgleichung"). Der dafür erforderliche numerische Aufwand ist aber wesentlich höher als im Fall eines reinen Verlustterms für die Strahlung und eignet sich schlecht für rasche Anpassungen von Modellen an beobachtete Daten, da das hydrodynamische Gleichungssystem und die Strahlungstransportgleichung nichttrivial gekoppelt sind. Um einfache Abschätzungen durchführen zu können, ist es also notwendig eine Näherung der Strahlungsverluste zu verwenden. Für die Zyklotronstrahlung definiert man analog zur Bremsstrahlungskühlzeit (Gl. 1.10):

$$
\begin{aligned}
& t_{\mathrm{cyc}}=\frac{3 k_{\mathrm{B}} T}{2 \mu m_{\mathrm{p}}}\left(\frac{1}{\rho} \underline{\partial} \cdot \underline{F}_{\mathrm{cyc}}\right)^{-1} \\
& \approx 1,8 \cdot 10^{2} \mathrm{~s} \frac{h}{10^{7} \mathrm{~cm}}\left(\frac{10^{7} \mathrm{~cm}}{d}\right)^{0,15}\left(\frac{10^{7} \mathrm{G}}{B}\right)^{2,85}\left(\frac{\rho}{\rho_{\mathrm{sh}}}\right)^{0,85} \\
& \qquad\left(\frac{T_{\mathrm{sh}}}{T}\right)^{1,5}\left(\frac{\dot{m}}{1 \frac{\mathrm{g}}{\mathrm{cm}^{2} \mathrm{~s}}}\right)^{0,85}\left(\frac{M_{\odot}}{M_{\mathrm{wd}}} \frac{R_{\mathrm{wd}}}{R_{\odot}}\right)^{1,925} .
\end{aligned}
$$


Der Energieverlust durch Zyklotronstrahlung muß hier - statt durch die spezifische Emissionsrate wie im optisch dünnen Fall - durch den spezifischen Strahlungsverlust $\left(\underline{\partial} \cdot \underline{F}_{\text {cyc }}\right) / \rho$ (Gl. 2.8) der Region beschrieben werden, um die optisch dicken Anteile mit einzubeziehen. Dazu wurde die Akkretionsregion als Kreiszylinder mit Radius $d$ und Höhe $h$ genähert und angenommen, daß der Zyklotronfluß $\underline{F}_{\text {cyc }}$ des Wasserstoffplasmas bis zu einer bestimmten Frequenz optisch dick ist und einem Schwarzkörperspektrum entspricht, während optisch dünne Anteile vernachlässigt wurden (siehe Kap. 2.2).

Ist Zyklotronstrahlung sogar der dominante Kühlprozeß, kann es erforderlich sein zwischen der Temperatur der Ionen und Elektronen zu unterscheiden, da bei einer starken Kühlung die Coulomb-Wechselwirkung nicht mehr effektiv genug ist, um über den gesamten geheizten Bereich eine gemeinsame Temperatur der Teilchen zu gewährleisten (Fabian, Pringle \& Rees 1976; Masters et al. 1976; Lamb \& Masters 1979). (Im folgenden wird mit „Zwei-Teilchen-Plasma“ die Unterscheidung der Elektronen- und Ionentemperatur und mit „Ein-Teilchen-Plasma“ der Fall $T_{\mathrm{e}} \equiv T_{\mathrm{i}}$ bezeichnet.) Eine Abschätzung des Parameterbereichs für diesen Fall ist durch die Betrachtung der beteiligten Zeitskalen möglich.

Wenn Elektronen und Ionen eine Maxwell-Boltzmann-Verteilung der Geschwindigkeiten zu den Temperaturen $T_{\mathrm{e}}$ und $T_{\mathrm{i}}$ besitzen, so werden Temperaturunterschiede in Zeiten von der Größenordnung der Äquipartitionszeit $t_{\mathrm{ei}}$ ausgeglichen (Spitzer 1962):

$$
\begin{aligned}
t_{\mathrm{ei}} & =\frac{3 m_{\mathrm{e}} m_{\mathrm{p}} k_{\mathrm{B}}^{\frac{3}{2}}}{8(2 \pi)^{\frac{1}{2}} n_{\mathrm{p}} e^{4} \ln \Lambda}\left(\frac{T_{\mathrm{i}}}{m_{\mathrm{p}}}+\frac{T_{\mathrm{e}}}{m_{\mathrm{e}}}\right)^{\frac{3}{2}} \\
& \approx 7,5 \cdot 10^{-11} \mathrm{~s}\left(\frac{T_{\mathrm{i}}}{T_{\mathrm{sh}}}+\frac{m_{\mathrm{p}}}{m_{\mathrm{e}}} \frac{T_{\mathrm{e}}}{T_{\mathrm{sh}}}\right)^{\frac{3}{2}} \frac{\rho_{\mathrm{sh}}}{\rho} \frac{1 \frac{\mathrm{g}}{\mathrm{cm}^{2} \mathrm{~s}}}{\dot{m}}\left(\frac{M_{\mathrm{wd}}}{M_{\odot}} \frac{R_{\odot}}{R_{\mathrm{wd}}}\right)^{2},
\end{aligned}
$$

mit $n_{\mathrm{p}}$ der Protonenanzahldichte $\left(\right.$ in $\left.\mathrm{cm}^{-3}\right), m_{\mathrm{e}}$ der Elektronenmasse, $e$ der Elementarladung und $\ln \Lambda \approx 10$ dem Coulomb-Logarithmus.

Ist nun die Zeitskala eines Kühlungsprozesses kleiner als diese Zeit, ist die Annahme einer gemeinsamen Temperatur für die Teilchen nicht mehr gerechtfertigt. Das Verhältnis von Äquipartitionszeit und Zyklotronkühlzeit ist:

$$
\begin{aligned}
\frac{t_{\mathrm{ei}}}{t_{\mathrm{cyc}}} \approx 4,0 \cdot 10^{-13} \frac{10^{7} \mathrm{~cm}}{h} & \left(\frac{d}{10^{7} \mathrm{~cm}}\right)^{0,15}\left(\frac{B}{10^{7} \mathrm{G}}\right)^{2,85}\left(\frac{\rho_{\mathrm{sh}}}{\rho}\right)^{1,85}\left(\frac{1 \frac{\mathrm{g}}{\mathrm{cm}^{2} \mathrm{~s}}}{\dot{m}}\right)^{1,85} \\
& \left(\frac{M_{\mathrm{wd}}}{M_{\odot}} \frac{R_{\odot}}{R_{\mathrm{wd}}}\right)^{3,925}\left(\frac{T_{\mathrm{i}}}{T_{\mathrm{sh}}}+\frac{m_{\mathrm{i}}}{m_{\mathrm{e}}} \frac{T_{\mathrm{e}}}{T_{\mathrm{sh}}}\right)^{1,5}\left(\frac{T_{\mathrm{e}}}{T_{\mathrm{sh}}}\right)^{1,5}
\end{aligned}
$$

und das Kriterium für die Unterscheidung der Teilchentemperaturen ist $t_{\mathrm{ei}} \geq t_{\mathrm{cyc}}$. Für einen weißen Zwerg mit $M_{\mathrm{wd}}=0,6 M_{\odot}$ und $B=30 \mathrm{MG}$ ist dies erfüllt, wenn $\dot{m} \leq 10 \mathrm{~g} \mathrm{~s}^{-1} \mathrm{~cm}^{-2}$, während bei $M_{\mathrm{wd}}=1,0 M_{\odot}$ der entsprechende Wert $40 \mathrm{~g} \mathrm{~s}^{-1} \mathrm{~cm}^{-2}$ ist. (Hier und durchweg im folgenden, wurde die Masse-Radius-Beziehung nach Nauenberg (1972) benutzt.) Selbst bei moderaten Magnetfeldstärken ist es also schon bei relativ hohen Massenstromdichten notwendig, eine Zwei-Teilchen-Beschreibung des Plasmas 


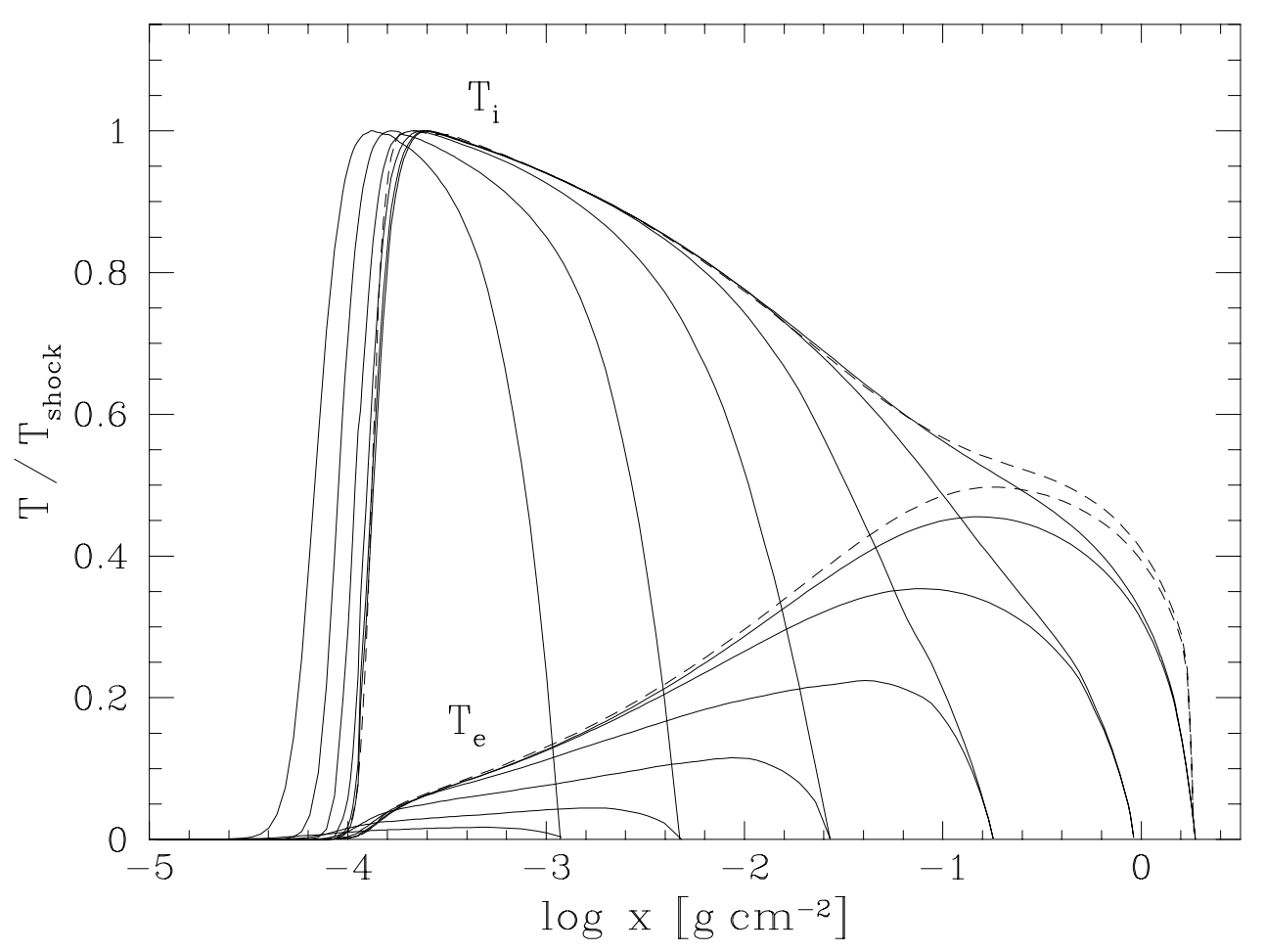

\begin{abstract}
Abbildung 1.4: Temperaturstruktur nach Zyklotron- und Bremsstrahlungskühlung als Funktion der Schichtdicke $x$ (aus Woelk \& Beuermann 1996). Dargestellt sind die Ionen- und Elektronentemperatur $T_{\mathrm{i}}, T_{\mathrm{e}}$ der Zwei-Teilchen-Beschreibung in Einheiten der Stoßtemperatur (Gl. 1.7 mit $\mu=1$ ) für $M_{\mathrm{wd}}=1,0 M_{\odot}$ und $B=$ 30 MG. Der steile Temperaturanstieg der Ionen kennzeichnet den Ort des Stoßes über dem Stern. Die Massenstromdichte $\dot{m}$ variiert von $10^{-3}$ bis $10^{2} \mathrm{~g} \mathrm{~s}^{-1} \mathrm{~cm}^{-2}$ in Schritten einer Größenordnung (durchgezogene Linien, von unten beginnend). Die gestrichelte Kurve entspricht reiner Bremsstrahlung $(B=0 \mathrm{MG})$ und ist unabhängig von $\dot{m}$.
\end{abstract}

durchzuführen. Rechnungen unter dieser Annahme ergeben einen Verlauf für die Elektronentemperatur, beginnend mit einem Temperaturanstieg am Anfang der Schicht, der durch die Coulomb-Wechselwirkung mit den heißen Ionen erzeugt wird. In der Nähe des Ortes der Äquipartition liegt ein ausgeprägtes Maximum, dessen Wert wegen der starken Kühlung deutlich unter der Stoßtemperatur der Ein-Teilchen-Beschreibung liegen kann, gefolgt von einer raschen Temperaturabnahme zur Oberfläche des weißen Zwergs hin. In Abbildung 1.4 ist ein solcher Temperaturverlauf für $T_{\mathrm{i}}$ und $T_{\mathrm{e}}$ als Funktion der Schichtdicke $x$ (in $\mathrm{g} \mathrm{cm}^{-2}$; mit $\mathrm{d} x:=-\rho \mathrm{d} z$ und der geometrischen Koordinate $z$ ) für verschiedene Massenstromdichten $\dot{m}$ dargestellt (Woelk \& Beuermann 1996). Im Gegensatz dazu zeigen Ein-Teilchen-Beschreibungen (Wu, Chanmugam \& Shaviv 1994) ein Temperaturmaximum mit dem Wert $T_{\mathrm{sh}}$ am Anfang der Schicht, gefolgt von der kontinuierlichen Abnahme durch Strahlungsverluste (für einen Vergleich siehe Kap. 3.5).

In Systemen, in denen der weiße Zwerg eine große Masse besitzt $\left(M_{\mathrm{wd}} \geq 1,0 M_{\odot}\right)$, beeinflussen weitere Prozesse die Struktur der Emissionsregion bzw. die beobachteten 
Spektren. Denn die von der Emissionsregion ausgehende Strahlung trifft zum Teil auf die Oberfläche des weißen Zwergs und erzeugt dort schwarzkörperartige UV- bzw. weiche Röntgenstrahlung, die bei einer hohen Masse des weißen Zwergs sehr intensiv sein kann. Die Elektronen im Gebiet hinter dem Stoß sind im Vergleich zur Strahlungsenergie dieser Komponente heiß $\left(k_{\mathrm{B}} T_{\mathrm{e}}>h \nu\right)$, wechselwirken mit den Photonen durch inverse Compton-Streuung und werden dabei gekühlt. In der Emissionsregion wirkt ComptonStreuung daher als zusätzlicher Kühlprozeß. Betrachtet man die Region vor dem Stoß, so hat Streuung den umgekehrten Effekt: Hier ist die Temperatur der Elektronen so gering, daß sie mit der harten Röntgenstrahlung der Emissionsregion durch Compton-Streuung wechselwirken und geheizt werden.

Als weiterer Prozeß tritt Wärmeleitung der Elektronen auf, die bei hohen Massen nicht mehr zu vernachlässigen ist (Imamura et al. 1987). Sie ist dominant in Regionen des Akkretionsstroms, in denen der Temperaturgradient groß ist, also im Gebiet vor dem Stoß und in der Nähe der Sternoberfläche. Ihre Auswirkung auf die Struktur des Akkretionsstroms ist ein Ausgleich der Elektronentemperatur durch Austausch von Energie benachbarter Regionen. Außerdem sorgt sie für eine Heizung des Plasmas vor dem Stoß, so daß die Elektronen eine höhere Temperatur als die Ionen haben (sog. „precursor"). In diesem Fall sind die einfachen Rankine-Hugoniot-Bedingungen (Gl. 1.5) für den Sprung der physikalischen Größen im Stoß nicht mehr anwendbar und müssen um den Wert der Elektronentemperatur im Bereich vor dem Stoß erweitert werden.

Ist das Gebiet vor dem Stoß zusätzlich optisch dick für die auftretende Strahlung, wird das ausgehende Spektrum noch durch Absorptionseffekte verändert und zeigt einen anderen Verlauf als das unbeeinflußte Spektrum der eigentlichen (dichteren) Emissionsregion.

Rechnungen in einer sphärisch symmetrischen Akkretionsgeometrie zeigen deutliche Unterschiede im Vergleich zu Rechnungen ohne die angesprochenen Prozesse (Kylafis \& Lamb 1979, 1982; Imamura et al. 1987). In Abbildung 1.5 (folgende Seite) ist eine Temperaturschichtung unter Berücksichtigung von Wärmeleitung und Compton-Kühlung für einen weißen Zwerg mit $M_{\text {wd }}=1,0 M_{\odot}$ und einer Akkretionsrate von $\dot{M}=9,2 \cdot 10^{19} \mathrm{~g} \mathrm{~s}^{-1}$ dargestellt (nach Imamura et al. 1987). Der Sprung in der Temperatur $T_{\mathrm{i}}$ der Ionen entspricht dem Ort des Stoßes, der hier als reine Diskontinuität aufgefaßt wird. Im Gebiet vor dem Stoß erstreckt sich der „precursor“ der geheizten Elektronen, deren mittlere Temperatur $T_{\mathrm{e}}$ bei den gewählten Parametern ca. $27 \%$ der Stoßtemperatur der Ionen erreicht. Bei Vernachlässigung der Wärmeleitung entspräche die Elektronentemperatur in diesem Gebiet der Stromtemperatur von einigen tausend Kelvin. Da Ionen und Elektronen durch die Coulomb-Wechselwirkung verbunden sind, werden auch die Ionen in diesem Gebiet schwach aufgeheizt. Nach dem Stoß gleichen sich die Temperaturen der beiden Teilchensorten an, und die Elektronen kühlen durch Bremsstrahlung und inverse Compton-Streuung. Zum Vergleich ist der Temperaturverlauf $T_{1}$ aus einer Ein-TeilchenRechnung angegeben. Die Elektronentemperatur der Zwei-Teilchen-Beschreibung bleibt unter diesen Werten, was sich im Spektrum der Emissionsregion bemerkbar macht. Weiterhin wird durch die Compton-Prozesse die Strahlung der Emissionsregion im harten Röntgenbereich abgeschwächt, während im weichen Röntgenbereich und im Ultraviolett die schwarzkörperähnlichen Komponenten verstärkt werden. Obwohl bei diesen Rechnun- 


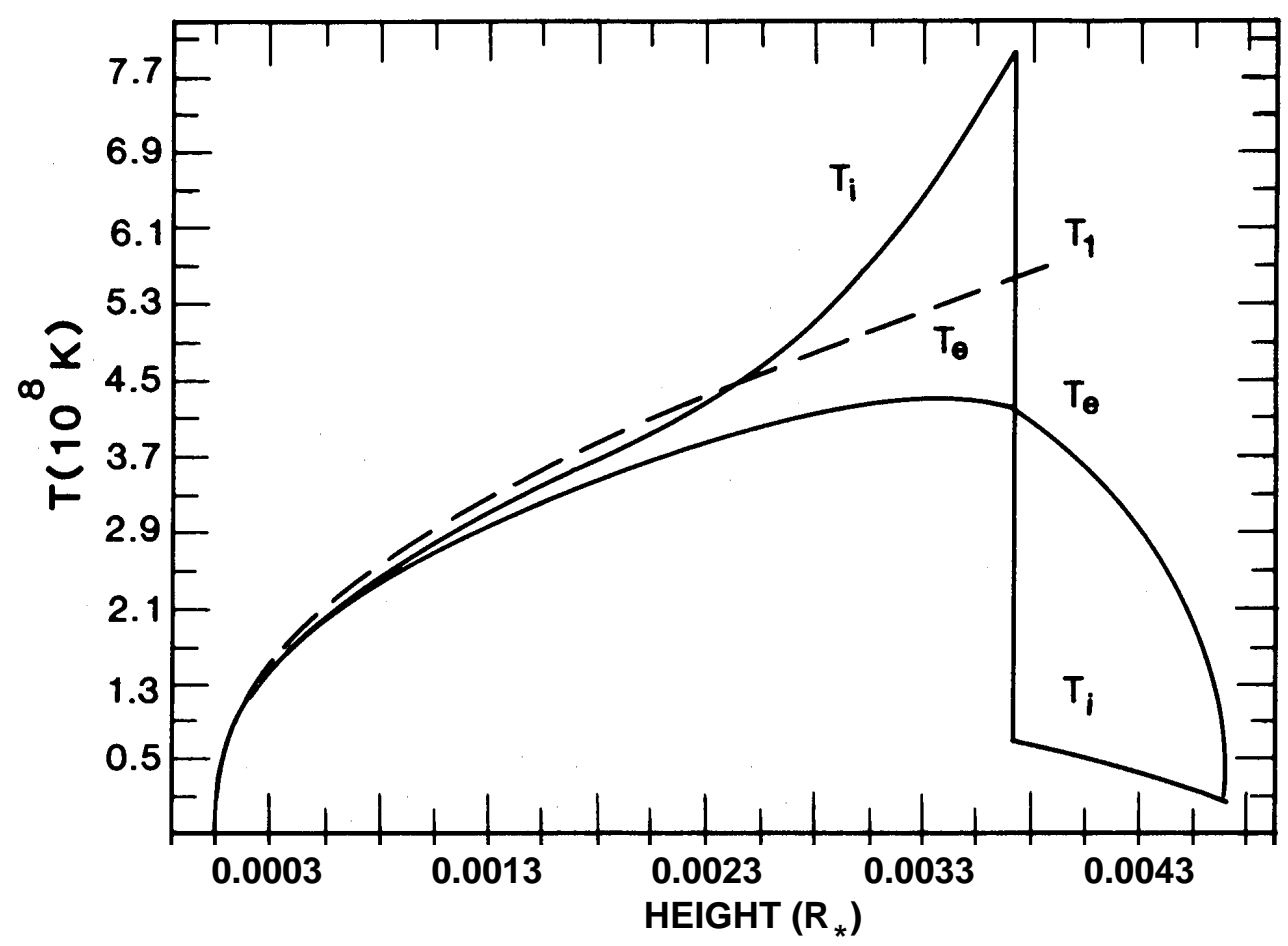

Abbildung 1.5: Temperaturstruktur nach Imamura et al. (1987) als Funktion der normierten Höhe über dem Stern. Dargestellt sind die Ionen- und Elektronentemperatur $T_{\mathrm{i}}, T_{\mathrm{e}}$ der Zwei-Teilchen-Beschreibung und der entsprechende Temperaturverlauf einer Ein-Teilchen-Rechnung $T_{1}$ (gestrichelte Linie) für $M_{\mathrm{wd}}=1,0 M_{\odot}$ und $\dot{M}=9,2 \cdot 10^{19} \mathrm{~g} \mathrm{~s}^{-1}$.

gen eine sphärische Geometrie zugrundegelegt wurde, sind die Ergebnisse für die Spektren auch anwendbar, wenn nur ein Teil der Sternoberfläche durch Akkretion geheizt wird. In diesem Fall zeigen niedrige, ausgedehnte Emissionsregionen, in die man hauptsächlich unter kleinen Winkeln zur Flächennormalen einsieht, ähnliche spektrale Verläufe wie bei der sphärischen Akkretion.

Eine obere Grenze für die Akkretionsrate ist erreicht, wenn die Kraft der erzeugten Strahlung auf die Elektron-Ion-Paare größer ist als die Gravitationskraft. Bei vollständiger Ionisation des Stroms und Akkretion auf einen Bruchteil $f:=A_{\mathrm{acc}} /\left(4 \pi R_{\mathrm{wd}}^{2}\right)$ der Sternoberfläche ergibt sich diese sog. „Eddington-Akkretionsrate“ aus der Gleichsetzung des Strahlungsdrucks auf die Elektronen infolge Thomsonstreuung und der Gravitationskraft auf die Ionen (resp. Protonen) zu:

$$
\dot{M}_{\mathrm{Edd}}=\frac{4 \pi c m_{\mathrm{p}}}{\sigma_{\mathrm{T}}} f R_{\mathrm{wd}}=6,6 \cdot 10^{22} \mathrm{~g} \mathrm{~s}^{-1} f \frac{R_{\mathrm{wd}}}{R_{\odot}},
$$

mit $c$ der Lichtgeschwindigkeit und $\sigma_{\mathrm{T}}$ dem Thomson-Wirkungsquerschnitt. Typische Werte für den Flächenbruchteil sind $f \geq 10^{-3}$ bei magnetisch kontrollierter Akkretion. Somit ist $\dot{M}_{\mathrm{Edd}} \approx 10^{18} \mathrm{~g} \mathrm{~s}^{-1}$ bzw. $\dot{m}_{\mathrm{Edd}} \approx 100 \mathrm{~g} \mathrm{~s}^{-1} \mathrm{~cm}^{-2}$. Für größere $\dot{M}$ ist eine stabile Akkretion nicht mehr möglich, da die Materie durch die Strahlung aufgehalten wird. 


\subsubsection{Zustände niedriger Akkretionsrate}

In „low-state“ Zuständen des Systems kann die Akkretionsrate so gering werden, daß der Akkretionsstrom nicht mehr als hydrodynamisches Fluid aufgefaßt werden darf, da sich die physikalischen Parameter über eine freie Weglänge der Stromteilchen ändern können. Stattdessen muß ein vollständig ionisiertes (Wasserstoff-)Plasma als aus freifallenden Einzelteilchen bestehend beschrieben werden. Eine Unterscheidung von Akkretionsstrom und Atmosphäre bezüglich der Partikel ist nur dadurch gegeben, daß die Teilchen des Stroms als "Test-Partikel" angesehen werden, die in eine thermische Verteilung von „Feldteilchen" der Atmosphäre eindringen. Mit Methoden der statistischen Plasmaphysik sind nun die Mechanismen der Wechselwirkung dieser Teilchen darzustellen.

Eine Abschätzung der Zeitskala der Abbremsung eines Teilchens in einem Plasma mit typischen Atmosphärentemperaturen, unter der Annahme von Coulomb-Wechselwirkung, zeigt, daß Elektronen instantan die Temperatur der Elektronen der Atmosphäre annehmen, während Protonen durch Stöße mit Protonen praktisch nicht abgebremst oder von ihrer Bahn abgelenkt werden, da diese Zeitskala größer ist als die für Proton-ElektronStöße (Woelk \& Beuermann 1992). Daher bringen die Protonen - wegen ihrer größeren Masse - bei der Abbremsung durch Coulomb-Stöße mit den Elektronen die akkretierte Energie in die äußeren Schichten der Atmosphäre ein, die somit aufgeheizt werden.

Bei hohen Massenstromdichten und schwacher Strahlungskühlung kann die eingebrachte Energie nicht effektiv genug wieder abgegeben werden. Es bildet sich ein Stoß aus, der die gerichtete Akkretionsenergie innerhalb einer freien Weglänge der Protonen thermalisiert. In einer magnetischen Atmosphäre ist - bei niedrigen $\dot{m}$ und/oder hohen Magnetfeldern - die Kühlung durch Zyklotronstrahlung allerdings schneller als der Energieaustausch zwischen Protonen und Elektronen, d. h., die Elektronen strahlen die gewonnene Energie innerhalb einer Wechselwirkungslänge rasch wieder ab, und die Entstehung eines Stoßes ist nicht mehr möglich. Dieses Szenario wird auch als „bombardment-solution“ bezeichnet (Kuijpers \& Pringle 1982; Thompson \& Cawthorne 1987; Woelk \& Beuermann 1992). Die Kühlung durch Zyklotronstrahlung ist in diesem Fall auch effektiver als durch Bremsstrahlung $\left(t_{\mathrm{cyc}}<t_{\mathrm{ff}}\right.$, Gl.1.12 bzw. Gl.11.10). Die Emission der geheizten Region besteht daher aus der dominanten Zyklotronkomponente, dem schwarzkörperähnlichen UV-Spektrum der Atmosphäre des weißen Zwergs und einem schwachen Bremsstrahlungsanteil.

Berechnungen der Temperaturstruktur des im Szenario der "bombardment-solution“ geheizten Gebiets, unter selbstkonsistenter Berücksichtigung des Energieverlusts der eindringenden Protonen und des Strahlungstransports in der Wasserstoffatmosphäre eines magnetischen weißen Zwergs, wurden von Woelk (1991; Woelk \& Beuermann 1992) durchgeführt. Nach einem kurzen Anstieg zeigt die Temperaturstruktur ein deutliches Maximum bei kleinen optischen Tiefen $\left(\tau_{\text {Ross }} \ll 1\right)$, gefolgt von einem raschen Temperaturabfall nachdem die Ionen ihre Energie vollständig abgegeben haben. Daran schließt eine schmale Region an, die durch Zyklotronstrahlung geheizt wird und in den Temperaturanstieg der Photosphäre übergeht (Abb. 1.6, folgende Seite). Für die Maximaltemperatur der Elektronen und die geometrische Tiefe $s$ der geheizten Schicht, angegeben als zugehörige 


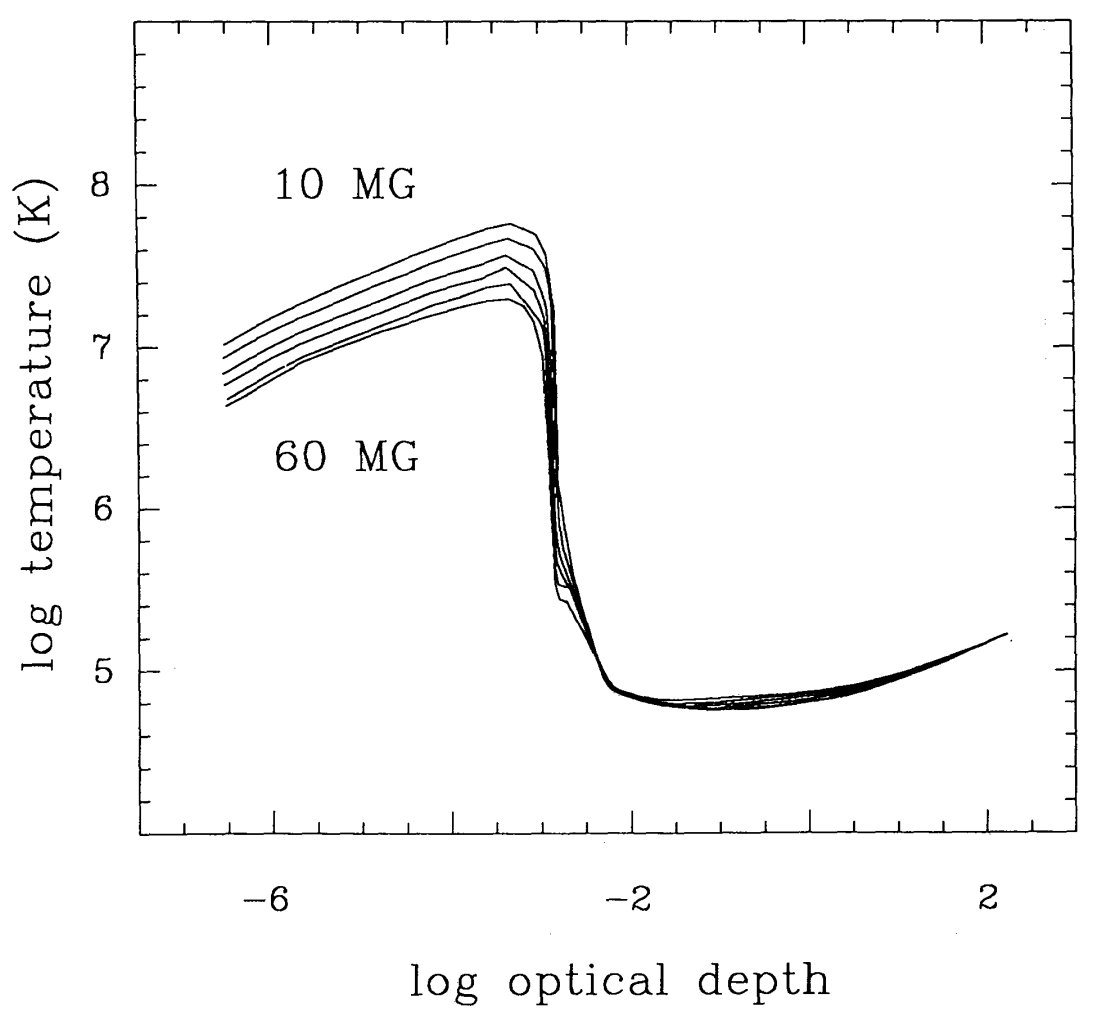

Abbildung 1.6: Temperaturstruktur einer teilchengeheizten, magnetischen Atmosphäre (aus Woelk \& Beuermann 1992). Aufgetragen ist die Elektronentemperatur gegen die Rosselandsche-optische Tiefe $\tau_{\text {Ross }}$ für $\dot{m}=10^{-3} \mathrm{~g} \mathrm{~cm}^{-2} \mathrm{~s}^{-1}$ und $M_{\mathrm{wd}}=0,7 M_{\odot}$, die Magnetfeldstärke $B$ variiert von $10 \mathrm{MG}$ (obere Kurve) bis $60 \mathrm{MG}$ (untere Kurve) in Schritten von $10 \mathrm{MG}$. Eine Variation von $\dot{m}$ und $M_{\mathrm{wd}}$ liefert ähnliche Strukturen.

Schichtdicke $x_{\mathrm{s}}$ (in $\mathrm{g} \mathrm{cm}^{-2}$ ), findet man folgende Abhängigkeiten (Woelk \& Beuermann 1993):

$$
\begin{aligned}
T_{\max } & =1,90 \cdot 10^{8} \mathrm{~K}\left(\frac{\dot{m}}{10^{-2} \frac{\mathrm{g}}{\mathrm{cm}^{2} \mathrm{~s}}}\right)^{0,42}\left(\frac{10^{7} \mathrm{G}}{B}\right)^{1,08}\left(\frac{M_{\mathrm{wd}}}{M_{\odot}}\right)^{0,66} \\
x_{\mathrm{s}} & =9,89 \cdot 10^{-3} \mathrm{~g} \mathrm{~cm}^{-2}\left(\frac{\dot{m}}{10^{-2} \frac{\mathrm{g}}{\mathrm{cm}^{2} \mathrm{~s}}}\right)^{0,30}\left(\frac{10^{7} \mathrm{G}}{B}\right)^{0,79}\left(\frac{M_{\mathrm{wd}}}{M_{\odot}}\right)^{1,72} \\
\text { mit } \quad x_{\mathrm{s}} & :=m_{\mathrm{p}} \int_{0}^{\mathrm{s}} n_{\mathrm{e}} \mathrm{d} r .
\end{aligned}
$$

Die Masse des weißen Zwergs und die Massenstromdichte bestimmen die in die Atmosphäre eingebrachte Energie, die Maximaltemperatur ist daher proportional zu diesen Größen. Die Magnetfeldstärke ist ein Maß für die Kühlung durch Zyklotronstrahlung: Große Magnetfelder erzeugen hohe Strahlungsverluste und verringern die Temperatur der Region. Die Ausdehnung der geheizten Schicht wird auf ähnliche Weise beeinflußt: Hoher Energieeintrag läßt die Schicht anwachsen, während starke Kühlung für eine Reduzierung der Ausdehnung sorgt. 


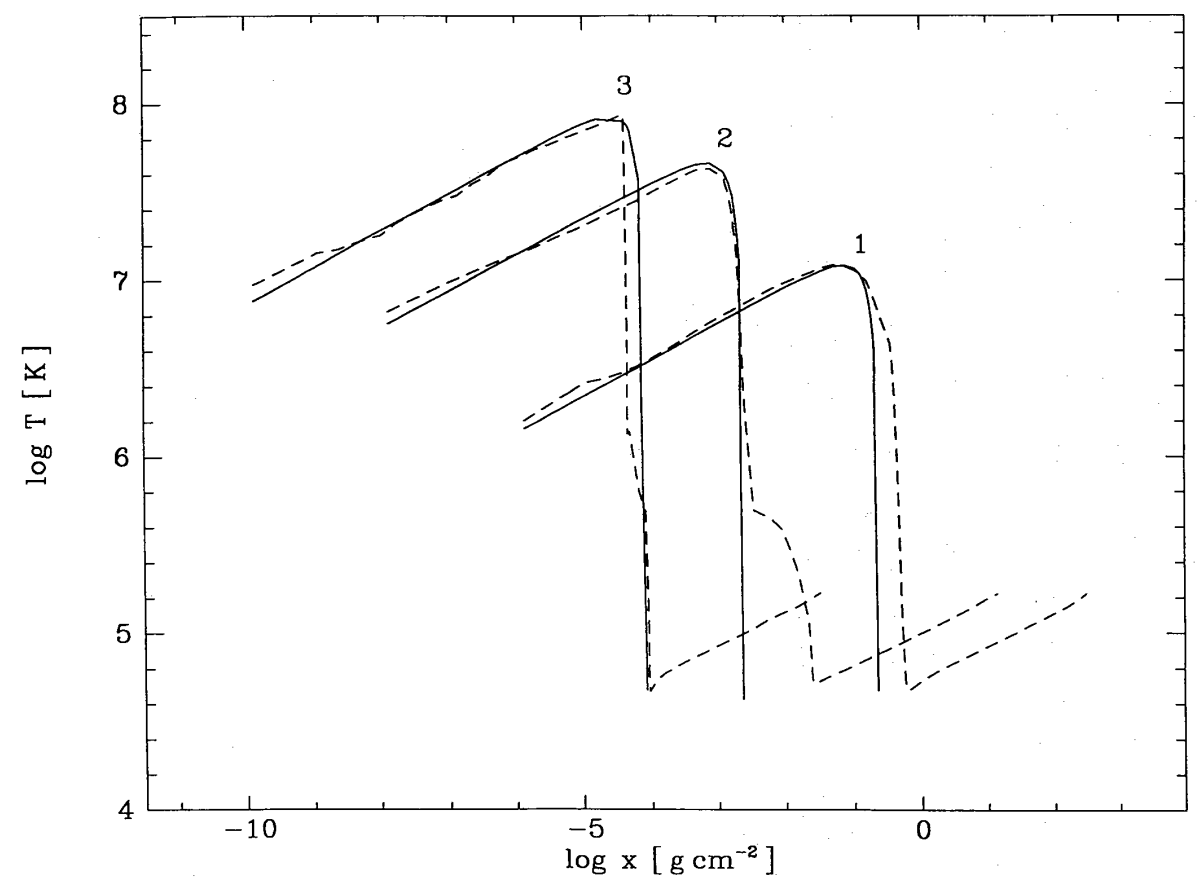

Abbildung 1.7: Parametrisierte Temperaturstruktur einer teilchengeheizten, magnetischen Atmosphäre (aus Woelk \& Beuermann 1993). Dargestellt ist die Elektronentemperatur aus Atmosphärenrechnungen (gestrichelte Linien) und nach der Parametrisierung (Gl.1.19, durchgezogene Linien) gegen die Schichtdicke der Atmosphäre für drei Parametersätze: (1) $\dot{m}=10^{-3} \mathrm{~g} \mathrm{~cm}^{-2} \mathrm{~s}^{-1}, B=60 \mathrm{MG}, M_{\mathrm{wd}}=$ $1,3 M_{\odot} ;(2) \dot{m}=10^{-2} \mathrm{~g} \mathrm{~cm}^{-2} \mathrm{~s}^{-1}, B=30 \mathrm{MG}, M_{\mathrm{wd}}=0,7 M_{\odot} ;(3) \dot{m}=$ $10^{-3} \mathrm{~g} \mathrm{~cm}^{-2} \mathrm{~s}^{-1}, B=10 \mathrm{MG}, M_{\mathrm{wd}}=1,3 M_{\odot}$. Die Kurven sind gegeneinander um zwei Größenordnungen in der Abszisse verschoben.

Normiert man die aus verschiedenen Werten von $M_{\mathrm{wd}}, \dot{m}$ und $B$ resultierenden Temperaturschichtungen auf ihre Maximalwerte $T_{\max }$ und $x_{\mathrm{s}}$, so stimmen die Verläufe im Bereich der geheizten Region grob überein, und eine Parametrisierung $T\left(x, M_{\mathrm{wd}}, \dot{m}, B\right)$ kann angegeben werden (Woelk \& Beuermann 1993):

$$
\log \left(\frac{T}{T_{\max }}\right)=a\left[\log \left(\frac{x}{x_{\mathrm{s}}}\right)-d\right]^{-2}+b\left[\log \left(\frac{x}{x_{\mathrm{s}}}\right)-d\right]+c,
$$

mit den aus Anpassungen an die Resultate der Atmosphärenrechnungen gefundenen Koeffizienten:

$$
\begin{aligned}
a & =-0,0374 \\
b & =0,2082 \\
c & =0,2220 \\
d & =0,2005 .
\end{aligned}
$$

In Abbildung 1.7 sind im Vergleich die Temperaturstrukturen aus den selbstkonsistenten Atmosphärenrechnungen und der parametrisierten Form nach Gleichung (1.19) für drei 
ausgewählte Parametersätze dargestellt. Die Übereinstimmung im Bereich der geheizten Region ist im mittleren Temperaturbereich ( $T \approx 10^{7} \mathrm{~K}$, Parametersatz 2$)$ sehr gut und für die anderen Bereiche im Rahmen der Genauigkeit der Rechnungen vertretbar. Mit solch einer Parametrisierung lassen sich in einer einfachen Weise Spektren der Akkretionsregion weißer Zwerge in einem Stadium geringer Massenflußraten $\left(10^{-3} \leq \dot{m} \leq 1 \mathrm{~g} \mathrm{~cm}^{-2} \mathrm{~s}^{-1}\right)$ berechnen, um Beobachtungsdaten zu interpretieren.

\subsection{5 Überschuß an weicher Röntgenstrahlung}

Nach dem oben beschriebenen Modell der stoßgeheizten Akkretionsregion wird die Oberfläche des weißen Zwergs durch Zyklotron- und Bremsstrahlung beleuchtet. Ungefähr die Hälfte der Gesamtstrahlungsenergie der Region sollte in der Atmosphäre reprozessiert werden und in Form einer schwarzkörperähnlichen, weichen Röntgen- bzw. UV-Komponente zum Gesamtspektrum beitragen. Zieht man weiterhin in Betracht, daß die Atmosphäre einen Teil der einfallenden harten Röntgenemission reflektiert, gilt für das Verhältnis von reprozessierter zu einfallender Strahlung:

$$
\frac{L_{\mathrm{bb}}\left(1-a_{\mathrm{X}}\right)}{L_{\mathrm{ff}}\left(1+a_{\mathrm{X}}\right)+L_{\mathrm{cyc}}} \approx 0,54,
$$

mit $a_{\mathrm{X}} \approx 0,3$ der Röntgenalbedo der Atmosphäre (Williams, King \& Brooker 1987; van Teeseling, Kaastra \& Heise 1996), $L_{\mathrm{bb}}$ der Leuchtkraft der reprozessierten Schwarzkörperstrahlung, $L_{\mathrm{ff}}$ der Leuchtkraft der harten Röntgenstrahlung in Form von Bremsstrahlung und $L_{\text {cyc }}$ der Zyklotronleuchtkraft (jeweils für den Halbraum definiert).

Tatsächlich beobachtet man in Polaren aber Verhältnisse, die diesen Wert beträchtlich überschreiten (siehe z.B. Ramsay etal. 1994), da eine starke Komponente im weichen Röntgenbereich auftritt. Diese Diskrepanz zwischen Theorie und Beobachtung wird in der Literatur als „Soft X-Ray Puzzle" bezeichnet, und eine Reihe von Erweiterungen und Alternativen des Modells wurden aufgestellt, von denen einige jedoch verworfen werden mußten: Nukleares Brennen (Raymond et al. 1979) oder Wärmeleitung der Elektronen in der Akkretionssäule (King \& Lasota 1980), Energieeintrag in die Atmosphäre durch Elektronen mit supra-thermischen Geschwindigkeiten (Frank, King \& Lasota 1983; Frank \& King 1984) und Beiträge aus der „bombardment-solution“ (Kuijpers \& Pringle 1982; Thompson, Brown \& Kuijpers 1986; Thompson \& Cawthorne 1987; Woelk \& Beuermann 1992).

Ein weiterer Ansatz zur Klärung des Problems geht von Inhomogenitäten des Akkretionsstroms aus, der nur dann als kontinuierlicher Materiefluß anzunehmen ist, wenn das Plasma in der Kopplungsregion mit dem Sternmagnetfeld ebenfalls homogen ist. Aber schon die Beobachtung von Zuständen unterschiedlicher Akkretionsrate und kurzzeitige Fluktuationen in der Strahlung (engl. "flickering") lassen darauf schließen, daß in dieser Region eine Vielzahl dynamischer Prozesse stattfindet, die den Strom beeinflussen. In Abbildung 1.8 sind solche kurzzeitigen Intensitätsschwankungen für Beobachtungen der Lichtkurve des Polars AM Herculis dargestellt. Das genaue Verständnis der Vorgänge, 


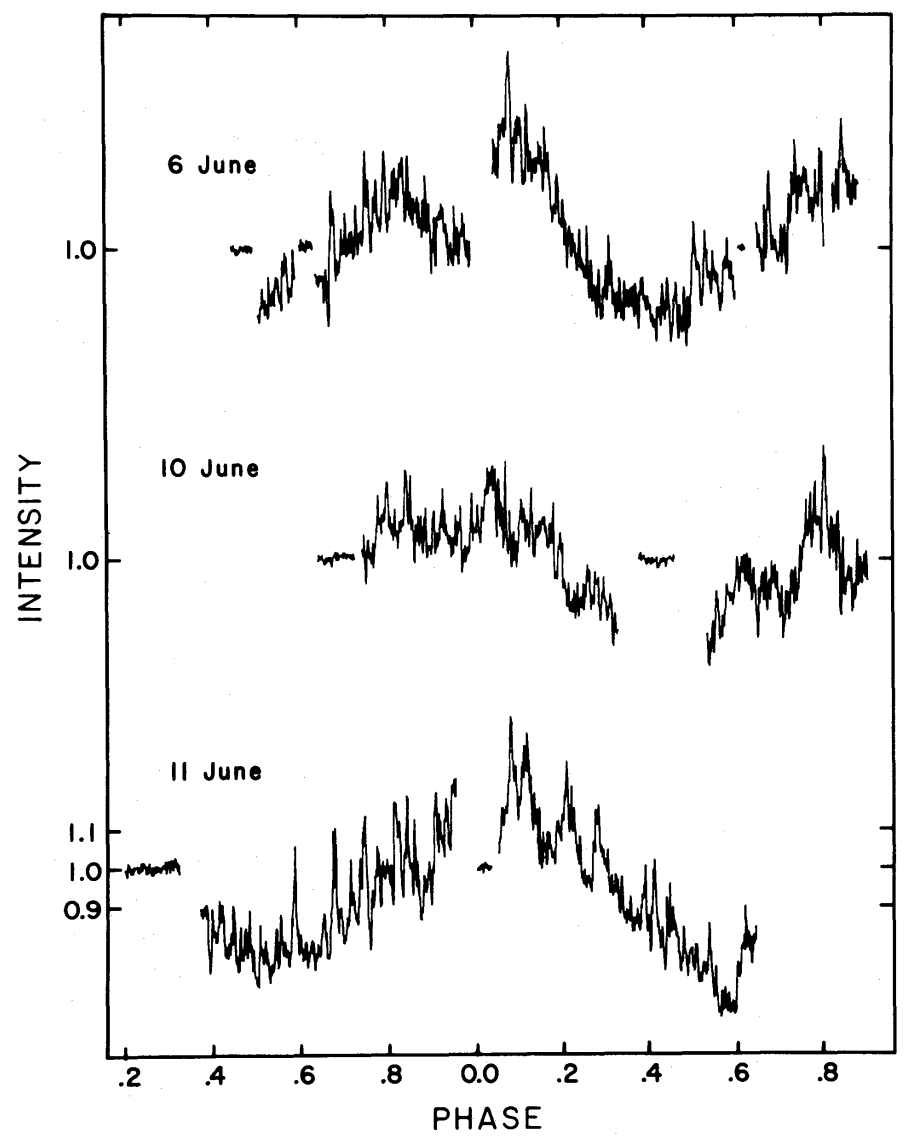

Abbildung 1.8: Optische Lichtkurve mit kurzzeitigen Intensitätsschwankungen des Polars AMHerculis (aus Panek 1980). Dargestellt ist die Intensität auf einer linearen Skala (mit versetztem Nullpunkt) gegen die Orbitalphase für drei Nächte im Jahr 1978. Die Zeitaufösung der Beobachtung entspricht 12,8s $\approx$ $10^{-3}$ Phaseneinheiten, die annähernd konstanten Bereiche mit Intensität um 1 zeigen den Vergleichsstern AM Her W und geben eine Abschätzung der systematischen Beobachtungsfehler.

die zu solchen Fluktuationen führen, steht noch aus. Jedoch zeigen Ergebnisse bisheriger Arbeiten, daß z. B. durch Instabilitäten (Rayleigh-Taylor und Kelvin-Helmholtz) der Strom in mehrere Fragmente unterschiedlicher Dichte aufgebrochen werden kann (Liebert \& Stockman 1985; Hameury, King \& Lasota 1986).

Kuijpers \& Pringle (1982) wiesen darauf hin, daß diese Inhomogenitäten beim Fall in den konvergierenden Magnetfeldlinien gestaucht und gestreckt werden und einzelne, dichte „Klumpen“ einen Staudruck erreichen können, dem ein Druck in großen optischen Tiefen der Atmosphäre entspricht, bis zu denen diese Fragmente einsinken. Damit wird die Akkretionsenergie in Bereichen freigesetzt aus denen die Strahlung nicht frei entweichen kann, somit von der umgebenen Materie absorbiert und in Form von Schwarzkörperstrahlung reemittiert wird. Dieser Anteil der Akkretionsrate trägt nicht zur Strahlung der Akkretionssäule bei, sondern nur zum reprozessierten, schwarzkörperähnlichen wei- 


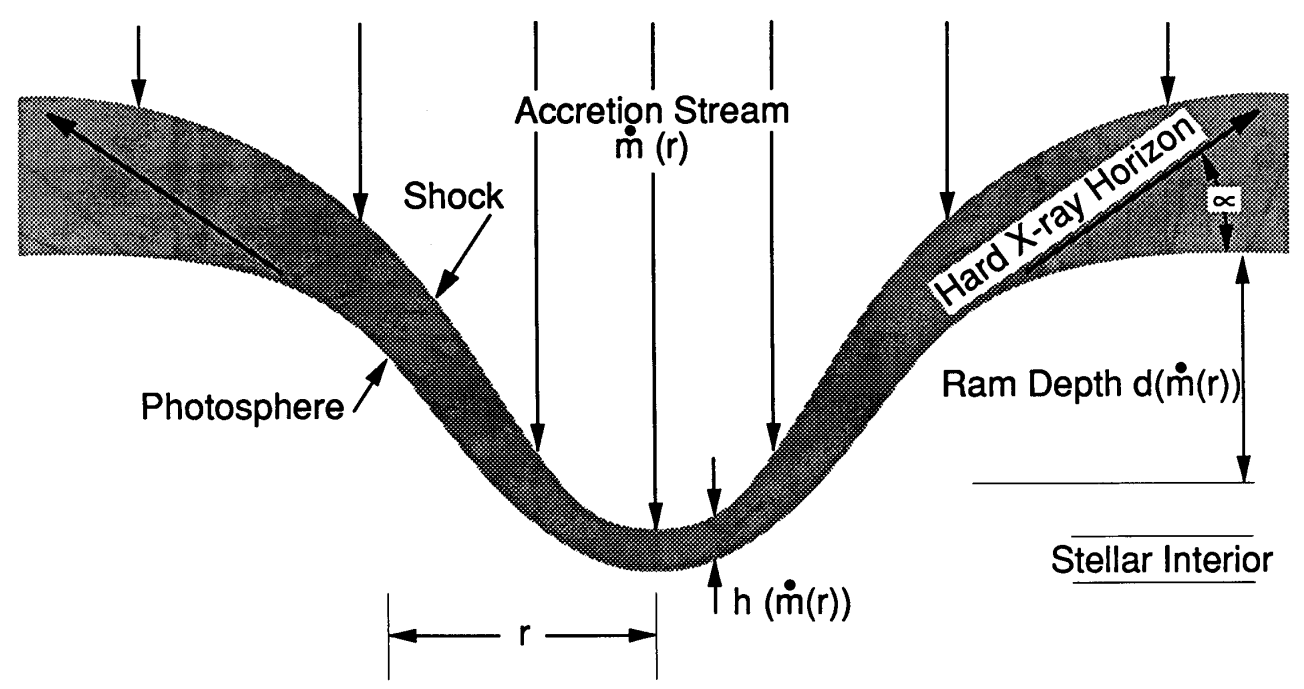

Abbildung 1.9: Querschnitt eines Akkretionsstroms mit radialer MassenflußVerteilung $\dot{m}(r)$ (aus Stockman 1988). Die Emissionsregion der lokalen Stoßhöhe $h(\dot{m}(r))$ sinkt bis zum Druckgleichgewicht mit der Photosphäre ein. Die hohen $\dot{m}$ im Kern ergeben eine starke Einsenkung, und die Strahlung wird im Material absorbiert.

chen Röntgen- bzw. UV-Spektrum und erhöht damit das Verhältnis $L_{\text {soft }} / L_{\text {hard }}$, wie es die Beobachtungen erfordern.

Detailliertere Rechnungen zu diesem Modell spezifizieren den Parameterbereich dieser sog. „blobby accretion“ (Frank, King \& Lasota 1988) und lassen es als Erklärung des „Soft X-Ray Puzzle " in AM Her-Systemen zu. Unter der Annahme, daß mehrere „Klumpen“ in Form von stoßgeheizten Akkretionssäulen zu verschiedenen, hohen Massenstromdichten innerhalb der Atmosphäre ihre Energie abstrahlen, lassen sich gute Anpassungen an Beobachtungen finden (Hameury \& King 1988; Litchfield \& King 1990). Eine konsistente Beschreibung der Energieverlustprozesse und Heizung in der Atmosphäre ist bisher allerdings nicht durchgeführt worden.

Die Beobachtung, daß Polare mit hohem Magnetfeld einen stärkeren Überschuß an weicher Röntgenstrahlung zeigen (Beuermann \& Schwope 1994; Ramsay et al. 1994; Beuermann \& Burwitz 1995), gibt einen weiteren Hinweis darauf, daß die größeren Massenflüsse einer $\dot{m}$-Verteilung den Anteil der einfallenden Materie bilden, der nur zur weichen Röntgenstrahlung beiträgt. Nimmt man eine konstante Akkretionsrate $\dot{M}$ in der Kopplungsregion an, dann skaliert die Massenstromdichte auf der Oberfläche des weißen Zwergs, in einem magnetischen Dipolfeld der Polfeldstärke $B$, mit $\dot{m} \sim B^{4 / 11}$. Bleibt das Verhältnis der einzelnen Massenstromdichten in einer $\dot{m}$-Verteilung zueinander konstant, wird bei einer Erhöhung der Feldstärke die Verteilung zu größeren $\dot{m}$ hin verschoben. Hohe Magnetfelder und große Massenstromdichten ergeben dann gering ausgedehnte Emissionsregionen (Woelk \& Beuermann 1996). Diese Regionen werden bis zu einer Tiefe in den weißen Zwerg eindringen, in der der Druck am Ende der Akkretionssäule und der lokale Atmosphärendruck vergleichbar sind. Ab einem gewissen $\dot{m}$ ist diese Einsinktie- 
fe größer als die Stoßhöhe, und die Emissionsregion ist vollständig in die Atmosphäre eingeschlossen.

Abbildung 1.9 zeigt schematisch die Gegebenheiten in einem Akkretionsstrom mit radialer $\dot{m}$-Verteilung. Die hohen $\dot{m}$ in der Strommitte weisen eine geringe Stoßhöhe $h(\dot{m})$ auf, werden wegen ihres höheren Staudrucks aber tiefer in die Atmosphäre gelangen. Die Strahlung aus diesem Gebiet wird daher stärker von der umgebenen Materie absorbiert, als dies in den Randgebieten des Akkretionsstroms geschieht, in denen $\dot{m}(r)$ einen kleineren Wert aufweist. Bei einer Vergrößerung der Magnetfeldstärke wird nun der Anteil der hohen $\dot{m}$ ebenfalls erhöht, und die Einsenkung des Akkretionsstroms in die Atmosphäre wird stärker. Damit sind ähnliche Verhältnisse wie im Modell der „blobby accretion“ gegeben, und der weiche Röntgenanteil wird zunehmen. Mit dieser Argumentation läßt sich das Verhältnis der Flüsse im harten und weichen Röntgenbereich $F_{\text {hard }} / F_{\text {soft }}$ als Funktion der Magnetfeldstärke $B$ für die bekannten Polare zumindest qualitativ beschreiben (Beuermann 1998).

\subsection{Zielsetzung und Aufbau der Arbeit}

In dieser Arbeit sollen Aspekte der Akkretion in magnetischen kataklysmischen Veränderlichen näher untersucht werden. Die bestehenden Ansätze zur Modellierung der Emissionsregion werden dazu auf ihren Gültigkeitsbereich hin überprüft, um Aussagen über deren Anwendbarkeit zu treffen. Die festgestellten Einschränkungen sollen, soweit dies möglich ist, innerhalb eines neuen Ansatzes behoben werden. An Hand von Beobachtungsdaten eines konkreten Polars ist der neue Ansatz zu testen und zu bewerten.

Um die Ziele zu erreichen, werden im Einleitungsteil der Gesamtzusammenhang der Akkretion und die grundlegenden Eigenschaften der magnetischen kataklysmischen Veränderlichen beschrieben. Das folgende Kapitel stellt die bestehenden Ansätze der Modellierung der Emissionsregion in Polaren dar und gibt eine Bewertung bezüglich der Anwendbarkeit auf konkrete Systeme. In Kapitel 3 wird ein Modell ausführlich in Bezug auf verwendete Annahmen und Näherungen dargestellt und die resultierende Emissionsregion für einen weiten Bereich der in Polaren auftretenden Parameter (Masse des weißen Zwergs, Massenstromdichte und Magnetfeldstärke) untersucht. Die sich dabei ergebenden Einschränkungen werden quantifiziert und ansatzweise in einem erweiterten Modell behoben. Das folgende Kapitel wendet die theoretischen Ergebnisse auf einen konkreten magnetischen kataklysmischen Veränderlichen (AM Herculis) an. Hier werden zum ersten Mal - durch die theoretische Interpretation der beobachteten Verteilungen des Energieflusses vom Infraroten bis in den harten Röntgenbereich - quantitative Aussagen über die Strukturierung der Massenstromdichten im Akkretionsfleck abgeleitet. Ein abschließendes Kapitel faßt die Arbeit zusammen und gibt einen Ausblick auf zukünftige Untersuchungen. 


\section{Kapitel 2}

\section{Hydrodynamisches Modell des Akkretionsstroms}

\subsection{Stationäres Ein-Teilchen-Plasma mit Brems- strahlungsverlusten}

Für kataklysmische Veränderliche in denen die akkretierende Komponente ein nur schwaches Magnetfeld besitzt, ist bei hohen Akkretionsraten Bremsstrahlung der dominante Strahlungsprozeß. Wegen der geringen optischen Tiefe der Emissionsregion für diese Strahlung wird somit Energie aus dem Akkretionsstrom heraus transportiert. Da die Equilibrierungszeitskala (Gl. 1.13) aber kleiner als die Kühlungszeitskala der Bremsstrahlung (Gl. 1.10) ist, gelangen Ionen und Elektronen durch Coulomb-Wechselwirkung zu einer gemeinsamen Temperatur. Es ist in diesem Fall also gerechtfertigt, das Plasma als Fluid mit nur einer Teilchenkomponente aufzufassen, da es hinsichtlich der Temperatur keine Unterscheidungsmöglichkeit zwischen Ionen und Elektronen gibt.

Eine einfache Annahme für den Akkretionsprozeß ist kontinuierliche, sphärisch symmetrische Akkretion eines vollständig ionisierten Plasmas ungefähr solarer Zusammensetzung mit den Massenanteilen des Wasserstoffs $X=0,7$ und des Heliums $Y=0$, 3, d.h. $\mu=0,615$ für die mittlere relative Teilchenmasse (Hoshi 1973). Die Emissionsregion wird durch die hydrodynamischen Gleichungen für Massen-, Impuls- und Energieerhaltung beschrieben:

$$
\begin{aligned}
r^{2} \rho v_{r} & =\frac{\dot{M}}{4 \pi} \\
v_{r} \frac{\mathrm{d} v_{r}}{\mathrm{~d} r}+\frac{1}{\rho} \frac{\mathrm{d} P}{\mathrm{~d} r}+\frac{G M_{\mathrm{wd}}}{r^{2}} & =0 \\
\rho v_{r} \frac{\mathrm{d} u}{\mathrm{~d} r}+\frac{P}{r^{2}} \frac{\mathrm{d}}{\mathrm{d} r}\left(r^{2} v_{r}\right)+\varepsilon_{\mathrm{ff}} \rho & =0,
\end{aligned}
$$

darin ist $\dot{M}$ die Akkretionsrate (in $\mathrm{g} \mathrm{s}^{-1}$ ), $v_{r}$ die Geschwindigkeitskomponente in radialer Richtung $r, \rho$ Massendichte, $P$ Druck, $u$ spezifische interne Energie $\left(u=\frac{3}{2} \frac{P}{\rho}\right.$ für einatomi- 
ge, ideale Gase), $\varepsilon_{\mathrm{ff}} \sim \rho T^{1 / 2}$ (Gl. 1.9) die spezifische Emissionsrate der Bremsstrahlung, $M_{\text {wd }}$ die Masse des weißen Zwergs und $G$ die Gravitationskonstante. Da Bremsstrahlung als optisch dünn in der Emissionsregion angenommen werden kann, läßt sich das Gleichungssystem bei vorgegebener Akkretionsrate und Masse numerisch einfach lösen. Als Randbedingung kann man einen starken Stoß über der Oberfläche des Sterns annehmen, denn der freifallende Plasmastrom erreicht Machzahlen größer als eins (Gl. 1.4).

Bei hinreichend hoher Akkretionsrate $\dot{M}$ ist die Kühlung des Plasmas groß genug, um die Höhe $h_{\text {sh }}$ der Stoßfront über der Sternoberfläche klein gegen den Sternradius $R_{\text {wd }}$ werden zu lassen. In diesem Fall ist es möglich, das obige Gleichungssystem durch einen Reihenansatz in dem Parameter $\xi:=h_{\mathrm{b}} / R_{\mathrm{wd}} \ll 1 \mathrm{zu}$ entwickeln und analytische Näherungslösungen für den Temperatur- bzw. Dichteverlauf von der Stoßfront bis zur Sternoberfläche anzugeben (Aizu 1973). Für die Stoßhöhe findet man

$$
h_{\mathrm{sh}}=\frac{2}{9 \sqrt{3}}(39 \sqrt{3}-20 \pi) h_{\mathrm{b}}=0,6053 h_{\mathrm{b}} .
$$

Die Kühlungslänge $h_{\mathrm{b}}$ ist die schon in Gleichung (1.11) definierte Abschätzung der Stoßhöhe, hier allerdings für $\mu=0,615$.

Die Geschwindigkeit als Funktion des Abstandes von der Stoßfront ist durch Umkehrung folgender Gleichung gegeben:

$$
\begin{aligned}
\frac{r-R_{\mathrm{wd}}-h_{\mathrm{sh}}}{R_{\mathrm{wd}}} \approx \frac{8}{9 \sqrt{3}} \xi & {\left[15 \arcsin \left(1-\frac{v_{r}}{2 v_{\mathrm{sh}}}\right)-\frac{5 \pi}{2}-\frac{39 \sqrt{3}}{4}+\right.} \\
+ & \left.\left(15+\frac{5 v_{r}}{2 v_{\mathrm{sh}}}+2\left(\frac{v_{r}}{v_{\mathrm{sh}}}\right)^{2}\right) \sqrt{\frac{v_{r}}{v_{\mathrm{sh}}}\left(1-\frac{v_{r}}{4 v_{\mathrm{sh}}}\right)}\right] .
\end{aligned}
$$

Für die Temperatur als Funktion der Geschwindigkeit erhält man

$$
\begin{aligned}
\frac{T}{T_{\mathrm{sh}}} \approx \frac{v_{r}}{3 v_{\mathrm{sh}}}\left(4-\frac{v_{r}}{v_{\mathrm{sh}}}\right) & +\xi \frac{64}{9 \sqrt{3}} \frac{v_{r}}{v_{\mathrm{sh}}}\left[\frac{\pi}{6}+\frac{13 \sqrt{3}}{16}-\arcsin \left(1-\frac{v_{r}}{2 v_{\mathrm{sh}}}\right)-\right. \\
& \left.-\left(1+\frac{v_{r}}{v_{\mathrm{sh}}}-\frac{1}{2}\left(\frac{v_{r}}{v_{\mathrm{sh}}}\right)^{2}+\frac{1}{8}\left(\frac{v_{r}}{v_{\mathrm{sh}}}\right)^{3}\right) \sqrt{\frac{v_{r}}{v_{\mathrm{sh}}}\left(1-\frac{v_{r}}{4 v_{\mathrm{sh}}}\right)}\right] .
\end{aligned}
$$

Die Dichteschichtung ergibt sich aus Kontinuitätsgleichung (Gl. 2.1) und implizitem Geschwindigkeitsverlauf (Gl. 2.5).

In Abbildung 2.1 ist der Temperatur-, Geschwindigkeits- und Dichteverlauf in Abhängigkeit des relativen Abstands zur Oberfläche des weißen Zwergs dargestellt. Die relative Stoßhöhe ist $\xi=0,1$, was einer Akkretionsrate von $\dot{M}=2,30 \cdot 10^{19} \mathrm{~g} \mathrm{~s}^{-1}=$ $3,63 \cdot 10^{-7} M_{\odot} \mathrm{a}^{-1}$ für einen weißen Zwerg mit Masse $M_{\mathrm{wd}}=1,0 M_{\odot}$ entspricht. Bei diesen Werten ist die Kühlzeitskala (Gl. 1.10) $t_{\mathrm{ff}}=0,31 \mathrm{~s}\left(T / T_{\mathrm{sh}}\right)^{1 / 2}\left(\rho / \rho_{\mathrm{sh}}\right)^{-1}$ größer als die Äquipartitionszeitskala (Gl. 1.13) $t_{\mathrm{ei}}=1,4 \cdot 10^{-2} \mathrm{~s}\left(T / T_{\mathrm{sh}}\right)^{3 / 2}\left(\rho / \rho_{\mathrm{sh}}\right)^{-1}$, und somit ist die Annahme einer gemeinsamen Temperatur von Ionen und Elektronen, trotz des Energieverlusts der Elektronen, gerechtfertigt. 


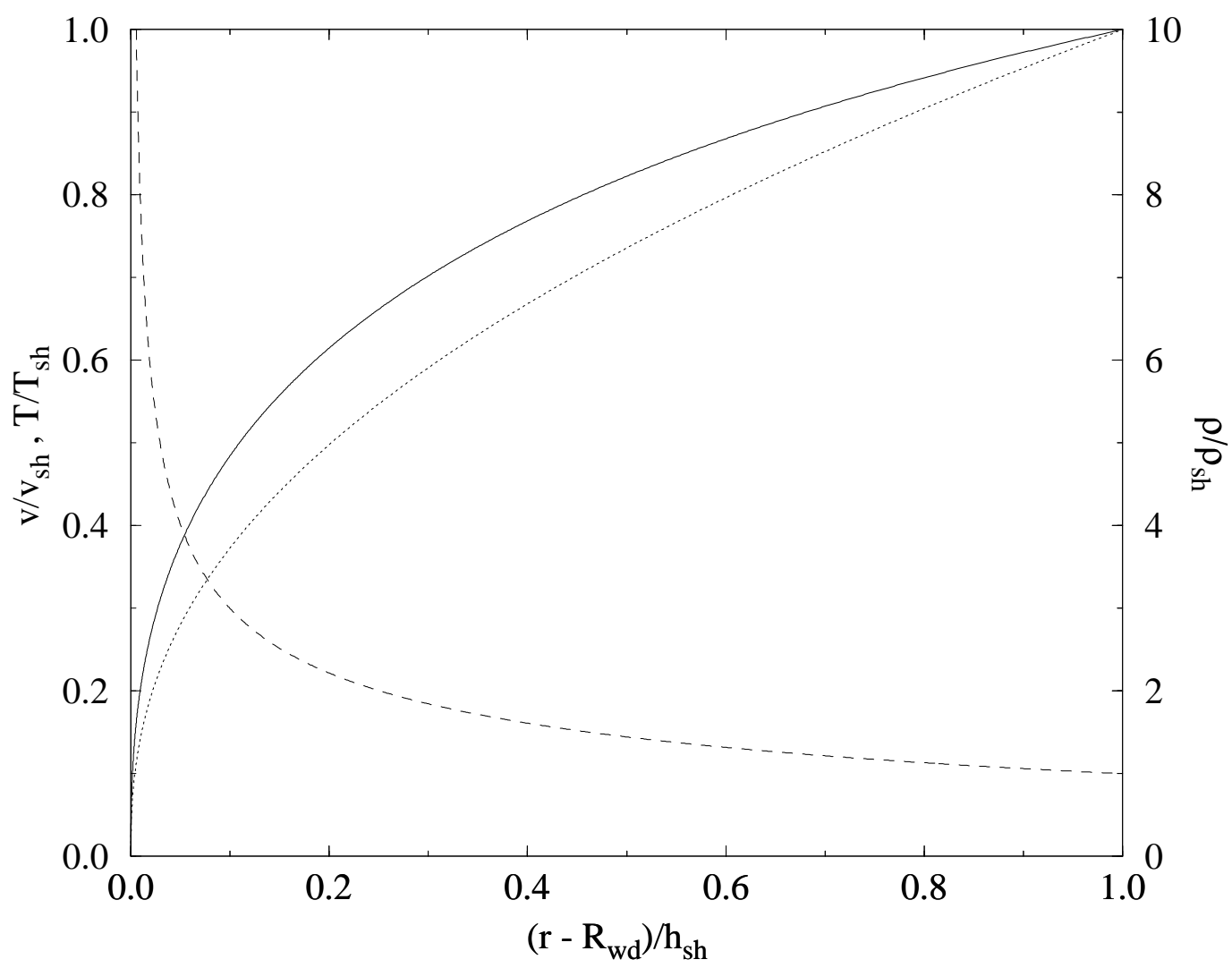

Abbildung 2.1: Analytische Näherungslösung der bremsstrahlungsgekühlten Emissionsregion nach Aizu (1973). Angegeben sind Temperatur (durchgezogene Linie), Geschwindigkeit (gepunktete Linie) und Dichte (gestrichelte Linie), normiert auf ihre Werte am Stoß, als Funktion der normierten Höhe. Der Entwicklungsparameter ist $\xi=0,1$.

Charakteristisch für die bremsstrahlungsdominierten Lösungen ist die langsame Temperaturabnahme vom Maximalwert: Erst nach ca. $90 \%$ der geometrischen Ausdehnung der Emissionsregion wird die halbe Stoßtemperatur erreicht. Der Grund dafür ist die Abhängigkeit des Kühlungsterms von $\rho^{2}$, der nur für große Dichten bedeutsam wird, und der durch die Kontinuitätsgleichung (Gl. 2.1) gegebene Dichteverlauf ist noch flacher als der Temperaturverlauf. Erst in der Nähe der Sternoberfläche wird die Dichte singulär und bewirkt dort eine entsprechend schnelle Temperaturänderung auf Null.

Obwohl die Lösung für sphärisch symmetrische Akkretion hergeleitet wurde, kann sie auch bei Akkretion auf einen kleinen Bruchteil $f$ der Sternoberfläche angewendet werden, wenn die Akkretionsrate $\dot{M}$ durch $\dot{m}=\dot{M} / f 4 \pi R_{\text {wd }}^{2}$ ersetzt wird und die sonstigen Annahmen (insbesondere $\xi:=h_{\mathrm{b}} / R_{\mathrm{wd}} \ll 1$ ) gültig bleiben. 


\subsection{Stationäres Ein-Teilchen-Plasma mit Brems- und Zyklotronstrahlungsverlusten}

Wird die Akkretion durch das Magnetfeld des Zentralsterns wesentlich beeinflußt, tritt zusätzlich zur Bremsstrahlung als weiterer Strahlungsprozeß Zyklotronstrahlung auf, die bei hohen Feldstärken den dominanten Anteil des Energieverlusts des Plasmas liefern kann. Da die Emissionsregion in diesem Fall sowohl optisch dünne als auch optisch dicke Bereiche aufweist, ist es nicht mehr möglich die exakten Strahlungsverluste als Funktion von Temperatur und Dichte in der Energiegleichung des Fluids darzustellen. Stattdessen ist es notwendig zusätzlich zu den hydrodynamischen Gleichungen auch den Strahlungstransport durch das Akkretionsplasma zu berechnen, um sowohl Energieverluste als auch Heizung durch die Strahlung richtig zu erfassen. Dies erfordert jedoch einen größeren numerischen Aufwand und ist daher für die rasche Beschreibung von Beobachtungsdaten oder für Untersuchungen zur Geometrie der Akkretionsregion unhandlich.

Unter Ausnutzung der Eigenschaften der Zyklotronstrahlung läßt sich der Strahlungstransport allerdings näherungsweise lösen, um damit das Gleichungssystem zu vereinfachen. Die Emissivität der Zyklotronstrahlung ist von der Frequenz, von Temperatur und Dichte des Plasmas und vom Winkel zwischen Ausbreitungsrichtung und Magnetfeld abhängig. Sie zeigt für eine thermische Verteilung der Elektronen eine harmonische Struktur, deren Einhüllende zu hohen Frequenzen hin stark abnimmt (Bekefi 1966).

In einem isothermen Plasma konstanter Dichte wird Zyklotronstrahlung daher bis zu einer Frequenz $\nu^{*}$ optisch dick sein und wie ein Schwarzkörper strahlen. Bei den zu erwartenden Temperaturen in der Größenordnung $T_{\mathrm{sh}} \approx 10^{8} \mathrm{~K}$ und Magnetfeldern $B \approx 10^{6} \mathrm{G}$ liegt die Zyklotronstrahlung im infraroten bis optischen Spektralbereich und man kann den Schwarzkörper durch das Rayleigh-Jeans-Gesetz approximieren. Für Frequenzen $\nu>\nu^{*}$ ist das Plasma optisch dünn und liefert nur noch einen geringen Beitrag zur Gesamtstrahlung. Bei bekannter Funktion $\nu^{*}(T, \rho)$ läßt sich somit der winkelgemittelte Verlust durch Zyklotronemission näherungsweise als Rayleigh-Jeans-Strahlung beschreiben, wenn man den Beitrag der optischen dünnen Anteile vernachlässigt.

Der problematische Teil dieser Näherung liegt nun in der Bestimmung von $\nu^{*}$, und eine Reihe von Rechnungen existieren in der Literatur, die je nach den verwendeten Annahmen und Näherungen zum Teil deutliche Unterschiede zeigen (ein kurzer Vergleich findet sich in Chanmugam \& Langer 1991). Hier soll nur die Formel von Wada et al. (1980) vorgestellt werden, da sie im weiteren verwendet werden wird. Die Autoren passen Ergebnisse von Chanmugam \& Wagner (1979) mit einer Potenzfunktion in $\rho d / B$ und $T$ an und finden

$$
\frac{\nu^{*}}{\nu_{\mathrm{c}}}=10,88\left(\frac{d}{10^{7} \mathrm{~cm}}\right)^{\frac{1}{20}}\left(\frac{10^{7} \mathrm{G}}{B}\right)^{\frac{1}{20}}\left(\frac{\rho}{10^{-8} \frac{\mathrm{g}}{\mathrm{cm}^{3}}}\right)^{\frac{1}{20}}\left(\frac{T}{10^{8} \mathrm{~K}}\right)^{\frac{1}{2}}
$$

mit $d$ dem Radius der Akkretionsregion, $B$ Magnetfeldstärke und $\nu_{\mathrm{c}}=e B /\left(2 \pi m_{\mathrm{e}} c\right)$ der Zyklotronfrequenz. 
Für eine Akkretionssäule der Höhe $h$ ist nun der spezifische, winkelgemittelte Strahlungsverlust durch den Zyklotronfluß $\underline{F}_{\text {cyc }}$ gegeben durch $\left(\underline{\partial} \cdot \underline{F}_{\text {cyc }}:=\operatorname{div} \underline{F}_{\text {cyc }}\right)$ :

$$
\frac{1}{\rho} \underline{\partial} \cdot \underline{F}_{\mathrm{cyc}} \approx \frac{8 \pi}{h \rho} \frac{2 \nu^{* 3}}{3 c^{2}} k_{\mathrm{B}} T \sim \frac{1}{\rho} \rho^{\frac{3}{20}} T^{\frac{5}{2}} .
$$

Damit ist eine einfache Lösung der hydrodynamischen Gleichungen ohne expliziten Strahlungstransport möglich. Diese Näherung ist nützlich, um sich eine Übersicht über das Lösungsverhalten zu verschaffen.

Von diesem Ergebnis ausgehend, setzen Wu, Chanmugam \& Shaviv (1994) eine Potenzfunktion in $P$ und $\rho$ als allgemeine Form für die Strahlungsverluste des Plasmas an. Sie führen einen Parameter $\eta_{\mathrm{sh}}$ ein, der das Verhältnis der Kühlungszeitskala für Bremsstrahlung zu der für Zyklotronstrahlung am Stoß angibt und lösen die stationären hydrodynamischen Gleichungen einer ebenen Akkretionsregion geringer Höhe unter der Annahme, daß Ionen und Elektronen dieselbe Temperatur besitzen. Das entsprechende Gleichungssystem der Erhaltungsgrößen lautet:

$$
\begin{aligned}
\rho v & =\dot{m} \\
\rho v \frac{\mathrm{d} v}{\mathrm{~d} h}+\frac{\mathrm{d} P}{\mathrm{~d} h} & =0 \\
v \frac{\mathrm{d} P}{\mathrm{~d} h}-\frac{5}{3} \frac{P}{\rho} v \frac{\mathrm{d} \rho}{\mathrm{d} h} & =-\frac{2}{3} C_{\mathrm{ff}} \rho^{2}\left(\frac{P}{\rho}\right)^{\frac{1}{2}}\left[1+\eta_{\mathrm{sh}}\left(\frac{P}{P_{\mathrm{sh}}}\right)^{\alpha}\left(\frac{\rho_{\mathrm{sh}}}{\rho}\right)^{\beta}\right],
\end{aligned}
$$

es ist $h$ die geometrische Höhenkoordinate der Schicht, $\dot{m}$ die konstante Massenstromdichte (in $\mathrm{g} \mathrm{s}^{-1} \mathrm{~cm}^{-2}$ ) und $C_{\mathrm{ff}}=3,9 \cdot 10^{16}$ cgs-Einheiten die Bremsstrahlungskonstante für ein vollständig ionisiertes Wasserstoffplasma $(\mu=1 / 2)$. Wenn $\eta_{\mathrm{sh}}=0$ oder die Dichte sehr groß ist, erhält man reine Bremsstrahlungskühlung. Im Fall der optisch dicken Zyklotronstrahlung - bei Verwendung des Ergebnisses von Wada et al. (1980) - werden die Parameter $\alpha$ und $\beta$ durch Vergleich mit dem genäherten Strahlungsverlust (Gl. 2.8) festgelegt: $\alpha=2, \beta=3,85$.

Unter der Annahme eines starken Stoßes läßt sich das Gleichungssystem zu einer Differentialgleichung für Geschwindigkeit und Höhe zusammenfassen (Wu 1994) und integrieren. Daraus folgt die Höhe des Stoßes über der Sternoberfläche $\left(\tau:=v / v_{\mathrm{ff}}\right)$ :

$$
\begin{aligned}
h_{\mathrm{sh}} & =\frac{v_{\mathrm{ff}}{ }^{3}}{2 C_{\mathrm{ff}} \dot{m}} \int_{0}^{1 / 4} \mathrm{~d} \tau \frac{\tau^{2}(5-8 \tau)}{[\tau(1-\tau)]^{1 / 2}} K(\tau) \\
\text { mit } \quad K(\tau) & :=\left[1+\left(\frac{4^{\alpha+\beta}}{3^{\alpha}}\right) \eta_{\mathrm{sh}}(1-\tau)^{\alpha} \tau^{\beta}\right]^{-1} .
\end{aligned}
$$

Der Geschwindigkeitsverlauf ist durch Umkehrung folgender Gleichung gegeben:

$$
\frac{h(\tau)}{h_{\mathrm{sh}}}=\frac{v_{\mathrm{ff}}{ }^{3}}{2 h_{\mathrm{sh}} C_{\mathrm{ff}} \dot{m}} \int_{0}^{\tau} \mathrm{d} \tau^{\prime} \frac{\tau^{\prime 2}\left(5-8 \tau^{\prime}\right)}{\left[\tau^{\prime}\left(1-\tau^{\prime}\right)\right]^{1 / 2}} K\left(\tau^{\prime}\right) .
$$




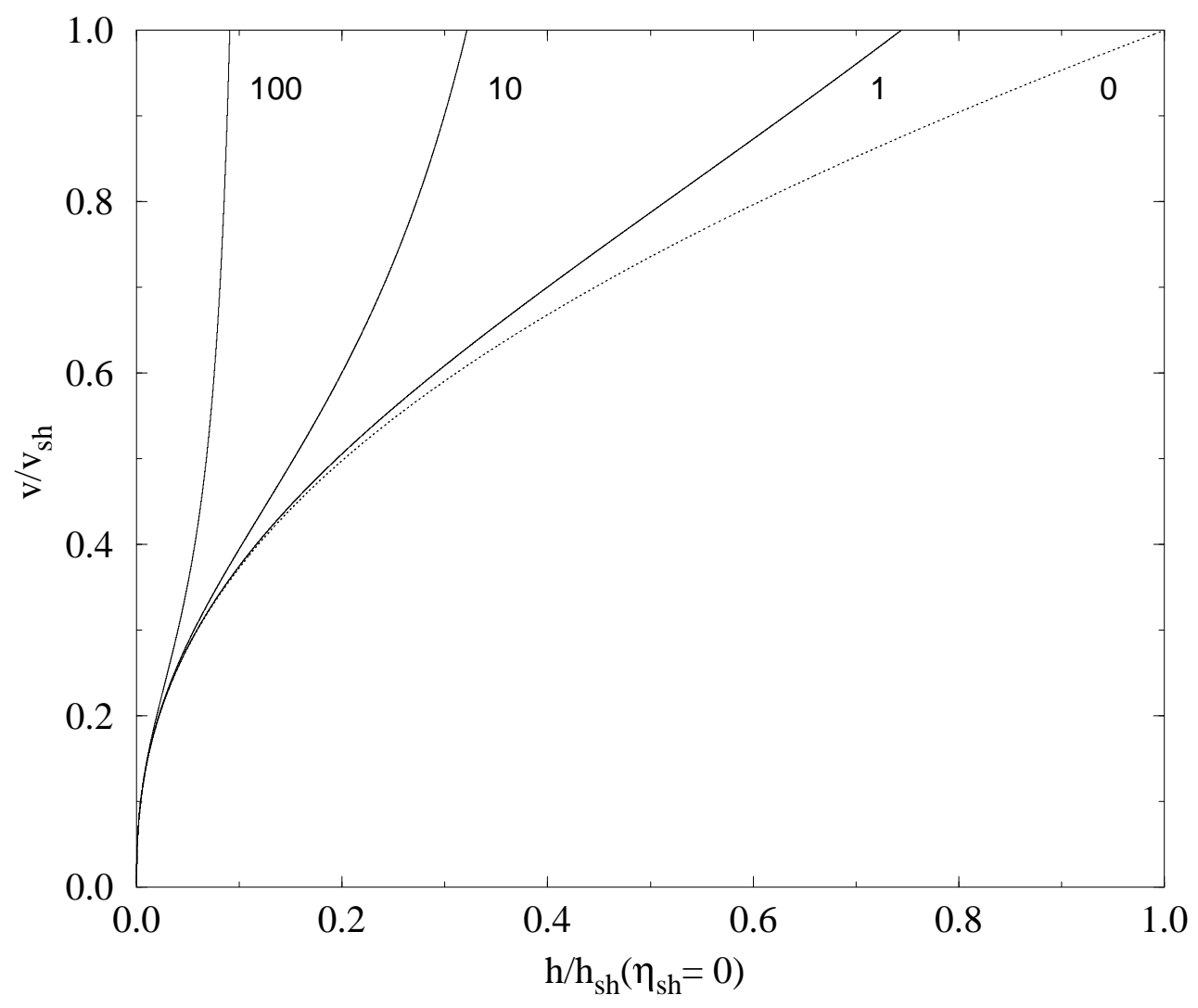

Abbildung 2.2: Geschwindigkeitsverlauf der Akkretionsregion als Funktion der Höhe nach Wu et al. (1994). Die Abszisse ist auf die Stoßhöhe bei reiner Bremsstrahlung $\left(\eta_{\mathrm{sh}}=0\right)$ normiert, in der Ordinate ist $v_{\mathrm{sh}}=v_{\mathrm{ff}} / 4$. Dargestellt ist der Verlauf für reine Bremsstrahlung (gepunktete Linie) und für zunehmende Zyklotronstrahlung (durchgezogene Linien mit $\left.\eta_{\mathrm{sh}}=1,10,100\right)$.

Dichte und Temperatur als Funktion der Geschwindigkeit werden beschrieben durch:

$$
\begin{aligned}
\frac{\rho}{\rho_{\mathrm{sh}}} & =\frac{1}{4 \tau} \\
\frac{T}{T_{\mathrm{sh}}} & =\frac{16}{3} \tau(1-\tau),
\end{aligned}
$$

mit $\rho_{\mathrm{sh}}=4 \dot{m} / v_{\mathrm{ff}}$.

Faßt man $\eta_{\text {sh }}$ als frei vorgegebenen Parameter auf, so lassen sich schnell quantitative Aussagen über das Verhalten der Emissonsregion bei zunehmender Dominanz der Zyklotronstrahlung machen. Wegen der Definition dieses Parameters als Verhältnis der Kühlungszeitskalen der beiden Strahlungsarten sind jedoch Gleichung (2.8) und Gleichung (2.12) gekoppelt und damit $\eta_{\mathrm{sh}}=f\left(h_{\mathrm{sh}}\right)$. Eine Abschätzung der Autoren ergibt:

$$
\eta_{\mathrm{sh}}=\left.\left(\frac{t_{\mathrm{ff}}}{t_{\mathrm{cyc}}}\right)\right|_{\mathrm{sh}} \approx 9,1 \cdot 10^{-3}\left(\frac{B}{10 \mathrm{MG}}\right)^{2,85}\left(\frac{T_{\mathrm{sh}}}{10^{8} \mathrm{~K}}\right)^{2}\left(\frac{10^{16} \mathrm{~cm}^{-3}}{n_{\mathrm{e}, \mathrm{sh}}}\right)^{1,85}\left(\frac{10^{7} \mathrm{~cm}}{h_{\mathrm{sh}}}\right)^{0,85}
$$




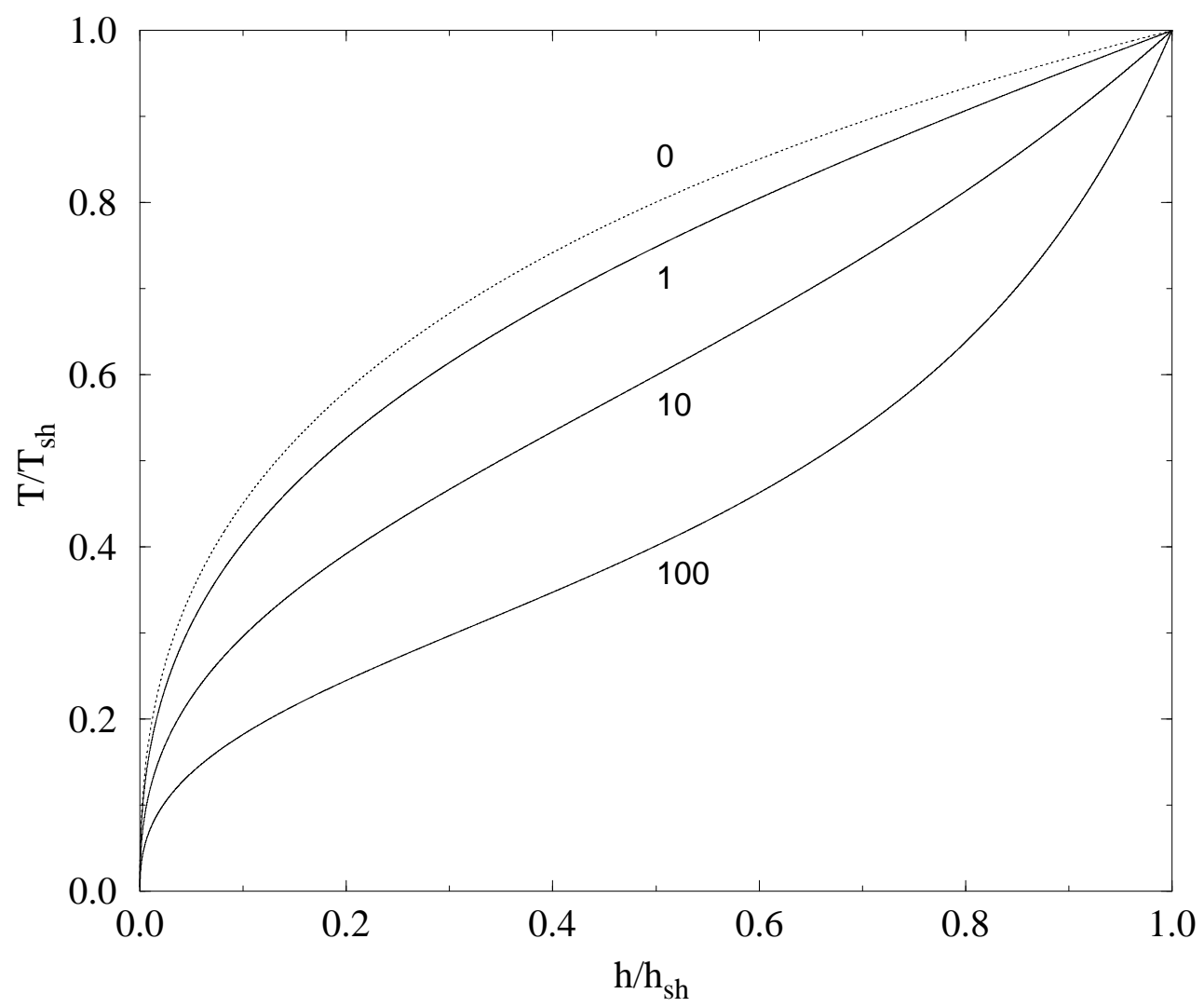

Abbildung 2.3: Temperaturverlauf der Akkretionsregion als Funktion der normierten Höhe nach Wu et al. (1994) für der Fall der reinen Bremsstrahlung (gepunktete Linie mit $\eta_{\mathrm{sh}}=0$ ) und zunehmender Zyklotronstrahlung (durchgezogene Linien mit $\left.\eta_{\mathrm{sh}}=1,10,100\right)$.

mit $n_{\mathrm{e}, \mathrm{sh}}$ der Elektronenanzahldichte am Stoß. Um einen Bezug zwischen $\eta_{\mathrm{sh}}$ und den stellaren Größen $B, M_{\mathrm{wd}}, \dot{m}$ herzustellen, ist es also notwendig eine zusätzliche unabhängige Bestimmung von $h_{\text {sh }}$ zu geben (z.B. numerische Lösung des Gleichungssystems) oder zwischen den Gleichungen (2.12) und (2.17) nach $h_{\mathrm{sh}} \mathrm{zu}$ iterieren.

In Abbildung 2.2 ist der Geschwindigkeitsverlauf in Abhängigkeit der Höhe dargestellt. Die Ordinate ist auf die Stoßhöhe für den Fall der reinen Bremsstrahlung $\left(\eta_{\mathrm{sh}}=0\right)$ normiert. Bei wachsendem Einfluß der Zyklotronstrahlung $\left(\eta_{\mathrm{sh}}=1,10,100\right)$ ist die Höhe der Emissionsregion gegenüber der Höhe bei reiner Bremsstrahlung erheblich reduziert. Schon bei gleicher Effizienz der beiden Strahlungen ist die Region um $20 \%$ weniger ausgedehnt.

Der Temperaturverlauf als Funktion der normierten Höhe ist in Abbildung 2.3 gezeigt. Hierbei wird die starke Abhängigkeit der Zyklotronstrahlung von der Temperatur deutlich (siehe Gl. 2.8). Bei hinreichend effizienter Zyklotronstrahlung ist die Temperatur kurz hinter dem Stoß schon deutlich abgesenkt, und die durchschnittliche Temperatur der Emissionsregion ist wesentlich kleiner als bei reiner Bremsstrahlungskühlung. Während im Fall der Bremsstrahlungskühlung die Hälfte der Stoßtemperatur erst nach ca. $90 \%$ der geometrischen Ausdehnung der Schicht erreicht ist, geschieht dies bei Parametern, 
bei denen die Zyklotronstrahlung 10 mal effektiver ist als Bremsstrahlung, schon nach ca. $60 \%$ der Ausdehnung.

Wenn die Verluste durch Zyklotronstrahlung so groß werden, daß die Elektronen ihre durch Coulomb-Wechselwirkung mit den Ionen gewonnene Energie wieder verlieren bevor sich eine Gleichgewichtsverteilung mit den Ionen gebildet hat, ist die Annahme der gleichen Temperatur für beide Teilchenkomponenten nicht mehr gerechtfertigt (Fabian, Pringle \& Ress 1976; Masters et al. 1977; Lamb \& Masters 1979). Das Kriterium hierfür ist $t_{\mathrm{ei}} \geq t_{\mathrm{cyc}}$, wenn $t_{\mathrm{cyc}}$ die Zeitskala der Zyklotronkühlung ist. Eine einfache Abschätzung (Gl. 1.14) ergibt

$$
\frac{t_{\mathrm{ei}}}{t_{\mathrm{cyc}}} \sim B^{2,85} \dot{m}^{-1,85} .
$$

Bei großen Magnetfeldstärken und/oder kleinen Massenflüssen ist also eine Unterscheidung zwischen Elektronen- und Ionentemperatur notwendig. So ist z. B. eine Kombination von $B$ und $\dot{m}$ möglich für die $\eta_{\text {sh }}=100$ in Abbildung 2.3 schon ein unzulässig hoher Wert ist, da die Plasmakomponenten keine gemeinsame Temperatur mehr erreichen. Genauere Aussagen über den Anwendungsbereich erfordern allerdings numerische Rechnungen, die das Verhalten der Zyklotronkühlung und die Geometrie der Emissionsregion besser modellieren können.

Die hier vorgestellte Beschreibung der magnetischen Akkretionsregion hat daher ihre Berechtigung, um schnell qualitative Aussagen zu finden. Für eine detailliertere Untersuchung der Akkretionsprozesse unter Berücksichtigung der Zyklotronstrahlung sind jedoch numerische Rechnungen nötig. Ein Vergleich mit solchen Rechnungen ist in Kapitel 3.5.2 durchgeführt.

\subsection{Instationäres Zwei-Teilchen-Plasma}

Die für Polare charakteristischen Lichtkurven werden durch den phasenabhängigen Sichtwinkel zur Akkretionsregion erklärt. Darüberhinaus beobachtet man in einigen Systemen aber noch kurzperiodische Veränderungen kleiner Amplitude des Flusses (Middleditch 1982; Larsson 1987, 1988, 1989; Middleditch, Imamura \& Steiman-Cameron 1997). Die Oszillationen treten über einen begrenzten Zeitraum mit konstanter Periode auf, die sich aber nach einer Anzahl von Zyklen ändern kann. Man nennt diese Helligkeitsschwankungen daher „Quasi-periodische Oszillationen“ (QPO). In Abbildung 2.4 ist eine photometrische Beobachtung hoher Zeitauflösung im sichtbaren Spektralbereich für den Polar V 834 Cen dargestellt, die eine deutliche Periodizität von $2 \mathrm{~s}$ aufweist. Die Amplitude des Flusses beträgt ca. 10\% eines Mittelwerts.

Die ersten Versuche zur Erklärung dieses Phänomens verwendeten Ergebnisse von Langer, Chanmugam \& Shaviv (1981, 1982), die erstmals zeitabhängige Rechnungen für Akkretion entlang der Magnetfeldlinien magnetischer weißer Zwerge durchführten. Sie fanden eine Instabilität der Akkretionsregion gegen radiale Störungen, die in einer Oszillation der Stoßhöhe resultiert. Unter der Annahme reiner Bremsstrahlung - mit einer 


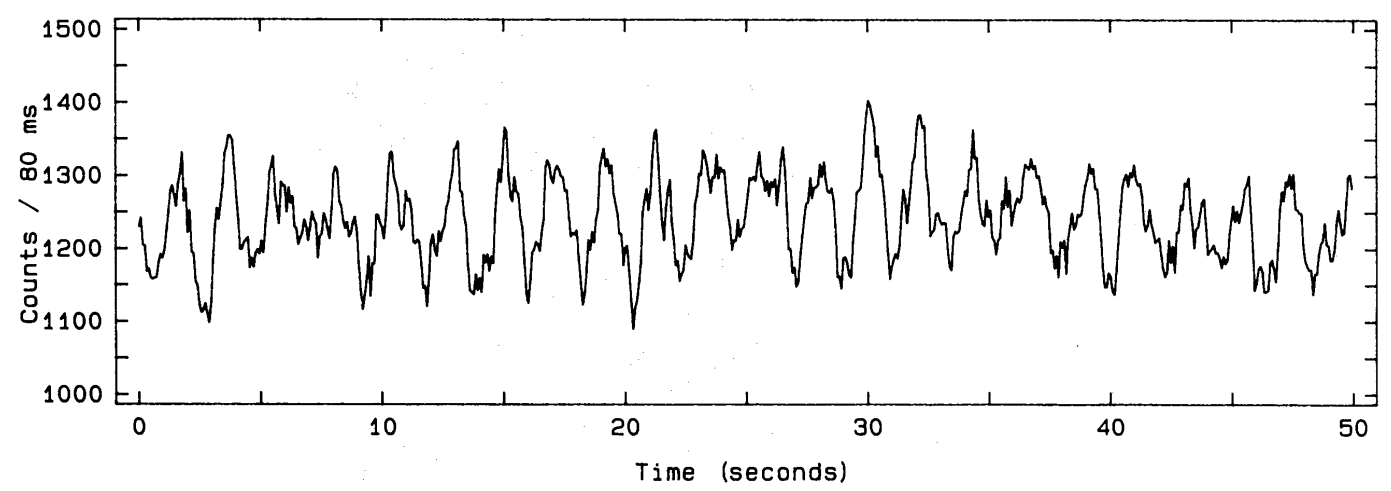

Abbildung 2.4: Quasi-periodische Oszillation des Polars V 834 Cen (aus Larsson 1992). Die optische Photometrie hoher Zeitaufösung (Mai 1984) zeigt eine deutliche Periode von $2 \mathrm{~s}$ und eine Variation des Flusses von ca. $10 \%$.

Kühlungsfunktion $\sim \rho^{2} T^{\alpha}($ mit $\alpha=1 / 2)$ in den hydrodynamischen Gleichungen - und der Identität von Elektronen- und Ionentemperatur kann die Oszillation als Wechselwirkung zwischen Kühlung des Gases durch Strahlung und Heizung durch Kompression verstanden werden. Bei Störung einer Gleichgewichtslösung zu größeren Höhen hin nimmt die Temperatur hinter dem Stoß zu, während die Dichte abnimmt. Die neue resultierende Höhe ergibt sich aus der Kühlzeit, die das Plasma benötigt, um die Randbedingung am Stern $(v \simeq 0)$ zu erfüllen. Bei einer Störung in Richtung der Oberfläche des weißen Zwergs nimmt die Stoßtemperatur ab, und die Dichte wird größer. Das Plasma kann jetzt effektiver kühlen, und die Abwärtsbewegung der Stoßregion wird verstärkt. Da aber gleichzeitig der mittlere Druck in der Region zunimmt, kann die Stoßhöhe nicht beliebig klein werden. Je nach Wahl der Randbedingung am Stern stellt sich somit eine minimale Höhe ein, und eine nach oben laufende Druckwelle kann den Stoß wieder nach außen treiben. Eine stabile Gleichgewichtslösung erfordert also einen Ausgleich zwischen Heizung durch einfallende Materie am Stoß und kühlendem Plasma am Boden. Eine von Langer et al. durchgeführte einfache Abschätzung zeigt, daß dies der Fall ist für eine Kühlungsfunktion mit $\alpha>-1 / 2$; ihre numerischen Ergebnisse liefern allerdings einen Wert von $\alpha \geq 1,6$, und nach einer verbesserten Version ihres Modells geben sie $\alpha \approx 0,6$ an (Chanmugam, Langer \& Shaviv 1985). Für kleinere $\alpha$-Werte stellt sich eine Oszillation mit einer Periode von der Größenordnung der Kühlzeit um eine Gleichgewichtslage ein.

Lineare (Chevalier \& Imamura 1982) und nichtlineare (Imamura, Wolff \& Durisen 1984) Stabilitätsanalysen des Problems zerlegen die Oszillationen in Moden mit zunehmender Frequenz: Grundfrequenz (F „fundamental"), 1. Oberschwingung (1O), 2. Oberschwingung (2O) usw. Die übereinstimmenden Resultate zeigen ein unterschiedliches Verhalten der einzelnen Moden. Während Stöße gegen Störungen mit Frequenzen der F-Mode stabil sind für $\alpha \geq 0,4$, gilt für die Moden $1 \mathrm{O}$ und $2 \mathrm{O}$ ein Grenze von $\alpha \geq 0,8$. Damit ist das Ergebnis von Langer et al. dahingehend relativiert, daß bremsstrahlungsdominierte Stöße stabil in der F-Mode sind, aber mit Frequenzen der $1 \mathrm{O}$ und $2 \mathrm{O}$ schwingen können. Als Beispiel für die Auswirkung des Parameters $\alpha$ auf die Oszillationen ist in Abbildung 2.5 (folgende Seite) die zeitliche Entwicklung der Stoßhöhe dargestellt (nach Wolff, Gard- 

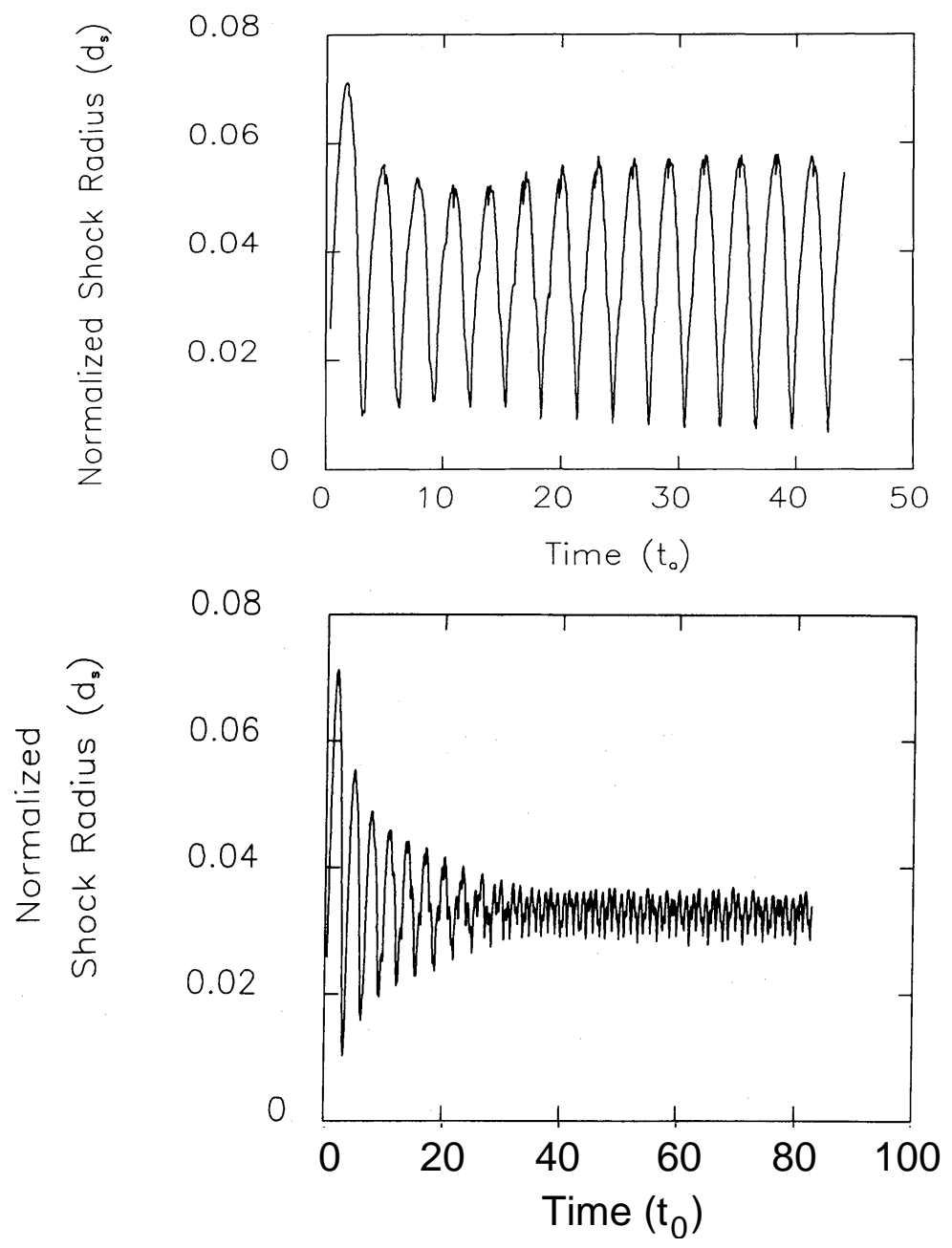

\begin{abstract}
Abbildung 2.5: Stoßhöhe als Funktion der Zeit nach Ein-Teilchen-Rechnungen von Wolff, Gardner \& Wood (1989) für eine weißen Zwerg der Masse $M_{\mathrm{wd}}=1,0 M_{\odot}$, mit einer Akkretionsrate von $\dot{M}=4,42 \cdot 10^{19} \mathrm{~g} \mathrm{~s}^{-1}$. Im oberen Teil ist $\alpha=1 / 3$, im unteren $\alpha=1 / 2$ entsprechend der reinen Bremsstrahlungslösung.
\end{abstract}

ner \& Wood 1989). Im unteren Teil ist die bremsstrahlungsdominierte Lösung mit $\alpha=1 / 2$ angegeben. Man erkennt deutlich die gedämpfte Grundschwingung (F) und nach deren Abklingen die erste Oberschwingung (1O). Eine Fourieranalyse ergibt noch Hinweise auf die zweite Oberschwingung (2O). Der obere Teil zeigt den Verlauf für $\alpha=1 / 3$. Hier ist das System für Störungen in allen Moden instabil und die Grundschwingung (F) dominiert die stabile Oszillation.

Das einfache Modell der Dominanz von Bremsstrahlung ist zur Beschreibung des Verhaltens der Akkretion in den meisten Polaren ungeeignet, da der Einfluß der Zyklotronstrahlung nicht ohne weiteres vernachlässigt werden kann. Weiterhin haben bei Systemen, in denen der weiße Zwerg eine hohe Masse aufweist, Wärmeleitung und ComptonKühlung Auswirkungen auf die Struktur der Akkretionsregion. Zudem ist es für eine korrekte Berücksichtigung dieser zusätzlichen Prozesse notwendig, zwischen der Temperatur 
von Elektronen und Ionen zu unterscheiden, also das hydrodynamische Gleichungssystem in einer Zwei-Teilchen-Beschreibung zu formulieren (siehe Kap. 1.2.3).

Während frühe Rechnungen für $T_{\mathrm{i}} \neq T_{\mathrm{e}}$ unter Berücksichtigung von Bremsstrahlung, Wärmeleitung und Compton-Kühlung, aber Vernachlässigung von Zyklotronstrahlung, einen stabilisierenden Effekt gegen radiale Störungen in allen Moden ergaben (Imamura, Wolff \& Durisen 1984; Wolff, Gardner \& Wood 1989), liefern neuere Rechnungen (noch ohne Wärmeleitung) Hinweise auf eine Destabilisierung für radiale Störungen, wenn die Temperatur der Teilchensorten im Stoß stark unterschiedlich ist (Imamura et al. 1996). Darüberhinaus zeigen diese Ergebnisse eine zusätzliche Instabilität gegen nichtradiale Störungen.

Die Einbeziehung der Zyklotronstrahlung in die Stabilitätsanalysen ist bisher nicht vollständig gelungen, da dies zeitabhängige, strahlungshydrodynamische Rechnungen erfordert. Stattdessen wird der optisch dicke Anteil der Strahlungsverluste näherungsweise in Form eines Rayleigh-Jeans-Spektrums bis zu einer kritischen Frequenz erfaßt (siehe Kap. 2.2). Entsprechende Rechnungen beschränken sich weiterhin noch auf die Hinzunahme von Bremsstrahlung und vernachlässigen die anderen oben angesprochenen Prozesse, so daß die Kühlungsfunktion in der Energiegleichung aus zwei Termen besteht und die Effizienz der Zyklotronstrahlung mit einem Parameter festgelegt werden kann. Die Resultate aus Ein-Teilchen-Rechnungen zeigen eine Stabilisierung gegen radiale Störungen in allen Moden für Magnetfelder größer als $B \approx 20 \mathrm{MG}$ (Chanmugam, Langer \& Shaviv 1985; Wu et al. 1996; Saxton, Wu \& Pongracic 1997; Saxton et al. 1998). Wenn Oszillationen auftreten, dann ist ihre Zeitskala nicht identisch mit der Kühlungszeitskala für Bremsstrahlung oder Zyklotronstrahlung, sondern weicht z. T. erheblich davon ab. Wu et al. (1992) führen dies auf eine unterschiedliche Dominanz der beiden Kühlprozesse zu verschiedenen Zeitpunkten der Oszillation zurück. Die Berücksichtigung von $T_{\mathrm{i}} \neq T_{\mathrm{e}}$ führt auch bei einem hohen Anteil an Zyklotronstrahlung zu einer Destabilisierung, und radiale sowie nichtradiale Oszillationen in Moden der Oberschwingungen können auftreten ( $\mathrm{Wu}$ \& Saxton 1999).

Nachdem die frühen Modelle mit befriedigendem Erfolg auf die wenigen vorhandenen Beobachtungen angewendet wurden, zeigten sich bald Schwierigkeiten und Inkonsistenzen bezüglich der Stabilität der Oszillationen. Alternative Modelle wurden aufgestellt, die mit ähnlichem Erfolg die Beobachtungen erklären konnten (z. B. Wolff, Wood \& Imamura 1991; Wood, Imamura \& Wolff 1992; Wu, Chanmugam \& Shaviv 1992; Steiman-Cameron et al. 1994). Jedoch lassen die neueren Ergebnisse zur Stabilität evtl. wieder eine Neuinterpretation im alten Kontext zu. Dafür ist es aber erforderlich, die einzelnen Entwicklungslinien zusammenzufassen und ein umfassenderes Modell als die oben beschriebenen aufzustellen.

Damit ist für die wenigen beobachteten kurzperiodischen Oszillationen bisher keine befriedigende Erklärung gefunden. Es scheint aber sicher, daß wenigstens die Grundschwingung (F) stabil ist, wenn weitere Kühlprozesse zur Bremsstrahlung hinzugenommen werden. Wenn sich also ein Stoß ausbildet, dann ist er im wesentlichen stabil und kann höchstens mit kurzperiodischen Oberschwingungen (1O, 2O, usw.) um eine Gleichgewichtslage oszillieren. 
In den oben zitierten Arbeiten wird aber auch deutlich, daß die korrekte Beschreibung der einzelnen Prozesse ausschlaggebend für die Aussage ist, ob und unter welchen Bedingungen Stöße stabil sind. Insbesondere ist der für die AM Her-Systeme wichtige Fall der hohen Magnetfelder und der daraus resultierenden Dominanz der Zyklotronstrahlung noch nicht zufriedenstellend betrachtet worden. In der vorliegenden Arbeit wird daher nicht weiter auf Oszillationen eingegangen werden, sondern auf die Auswirkungen der Zyklotronstrahlung auf die Struktur der Emissionsregion unter der Annahme der Existenz einer (quasi-)stationären Lösung der strahlungshydrodynamischen Gleichungen. 


\section{Kapitel 3}

\section{Stationäre Strahlungshydrodynamik}

\subsection{Grundgleichungen}

In der Näherung als hydrodynamisches, viskoses Fluid wird der ionisierte Akkretionsstrom durch die Erhaltungsgleichungen für Masse, Impuls und Energie dargestellt. Wegen der starken elektrischen Wechselwirkung zwischen Ionen und Elektronen ist zur Beschreibung des Verhaltens des Stroms eine Bewegungsgleichung für diese Teilchenpaare ausreichend. Dagegen soll berücksichtigt werden, daß die Teilchen eine unterschiedliche Temperatur annehmen können und, daß das Fluid durch Strahlung beeinflußt wird („Strahlungshydrodynamik"). Das resultierende Gleichungssystem lautet:

- Kontinuitätsgleichung:

$$
\frac{\partial \rho}{\partial t}+\underline{\partial} \cdot(\rho \underline{v})=0
$$

- Navier-Stokes-Gleichung:

$$
\frac{\partial \underline{v}}{\partial t}+(\underline{v} \cdot \underline{\partial}) \underline{v}-\underline{g}-\frac{1}{\rho}(\underline{\underline{\sigma}} \cdot \underline{\partial})=0 .
$$

- Energiegleichung der Ionen bzw. Elektronen:

$$
\begin{aligned}
\frac{\partial}{\partial t} E_{\mathrm{i}}+\underline{\partial} \cdot\left(E_{\mathrm{i}} \underline{v}\right)-\underline{\partial} \cdot(\underline{\underline{\sigma}} \cdot \underline{v})-\underline{\partial} \cdot\left(P_{\mathrm{e}} \underline{v}\right)-\rho \underline{g} \cdot \underline{v}+\Lambda_{\mathrm{x}}=0 \\
\frac{\partial}{\partial t} E_{\mathrm{e}}+\underline{\partial} \cdot\left(E_{\mathrm{e}} \underline{v}\right)+\underline{\partial} \cdot\left(P_{\mathrm{e}} \underline{v}\right)-\rho \underline{g} \cdot \underline{v}-\Lambda_{\mathrm{x}}+\underline{\partial} \cdot \underline{Q}+\underline{\partial} \cdot \underline{F}_{\mathrm{rad}}=0 .
\end{aligned}
$$

Darin ist $\underline{g}$ die Schwerebeschleunigung und $\underline{\underline{\sigma}}$ der Spannungstensor mit den Komponenten $(j, k=1, \overline{2}, 3)$

$$
\sigma_{j k}:=-\left(P_{\mathrm{e}}+P_{\mathrm{i}}\right) \delta_{j k}+\zeta\left(\partial_{j} v_{k}+\partial_{k} v_{j}\right)+\left(\xi-\frac{2}{3} \zeta\right) \underline{\partial} \cdot \underline{v} \delta_{j k},
$$


mit $\zeta$ der dynamischen Viskosität (1. Lamé-Konstante; in $\left.\mathrm{g} \mathrm{cm}^{-1} \mathrm{~s}^{-1}\right), \xi$ der Volumenviskosität (2. Lamé-Konstante), $\delta_{j k}$ (1 für $j=k ; 0$ sonst) dem Kronecker-Symbol und $\partial_{j}$ der j-ten Komponente des Differentialoperators "Nabla“ $\underline{\partial}:=\left(\frac{\partial}{\partial x_{1}}, \frac{\partial}{\partial x_{2}}, \frac{\partial}{\partial x_{3}}\right)$. Die Ionen- bzw. Elektronenenergie pro Volumenelement des Fluids ist $E_{\mathrm{i}, \mathrm{e}}:=\frac{1}{2} \rho v^{2}+\rho u_{\mathrm{i}, \mathrm{e}}$, wobei $u_{\mathrm{i}, \mathrm{e}}$ die spezifische interne Energie der Ionen bzw. Elektronen ist.

Die Viskosität geht in die Bewegungsgleichung (Gl. 3.2) und in die Energiegleichung der Ionen ein; die Elektronen erfahren wegen ihrer geringen Masse kaum Energieverluste durch Reibung.

Der Energieaustausch durch Coulomb-Wechselwirkung zwischen Ionen und Elektronen ist in einem vollionisierten, einatomigen, idealen Gas gegeben durch (Spitzer 1962):

$$
\Lambda_{\mathrm{x}}=\frac{3}{2} \frac{n_{\mathrm{e}} k_{\mathrm{B}}\left(T_{\mathrm{i}}-T_{\mathrm{e}}\right)}{t_{\mathrm{ei}}},
$$

mit der Äquipartitionszeit $t_{\text {ei }}$ (Gl. 1.13).

Durch Wärmeleitung der Elektronen entsteht ein Energiefluß $Q$, der in einem vollionisierten Wasserstoffplasma mit Coulomb-Logarithmus $\ln \Lambda \approx 10$ beschrieben ist durch (Zel'dovich \& Raizer 1966):

$$
\underline{Q}=-1,83 \cdot 10^{-6} \operatorname{erg~s}^{-1} \mathrm{~cm}^{-1} \mathrm{~K}^{-\frac{7}{2}} T_{\mathrm{e}}^{\frac{5}{2}} \underline{\partial} T_{\mathrm{e}} .
$$

Die Verluste durch Strahlung aus der betrachteten Region werden durch den Term $\underline{\partial} \cdot \underline{F}_{\text {rad }}$ erfaßt, wenn $\underline{F}_{\text {rad }}$ der Strahlungsfluß ist. Er geht nur in die Energiegleichung der Elektronen ein, da angenommen wird, daß die Ionen (wegen ihrer größeren Masse) im Vergleich zu den Elektronen einen verschwindenden Beitrag zur Emission liefern. Kann die Strahlung die Region ohne Wechselwirkung mit der Materie verlassen („optisch dünne“ Näherung), ist dieser Term gleich der über einen Raumwinkel gemittelten, frequenzintegrierten Emissivität (in $\mathrm{erg} \mathrm{s}^{-1} \mathrm{~cm}^{-3}$ ) für den betrachteten Strahlungsprozeß und bei einigen Strahlungsarten eine einfache Abhängigkeit von Dichte $\rho$ und Temperatur T. Auch der Grenzfall der totalen Absorption innerhalb des Plasmas („optisch dicke“ Näherung) ist mit einer einfachen Formel darstellbar (Rosseland-Approximation, Diffusions-Näherung). Hier verhält sich die Strahlung in der Art des Energietransports durch Wärmeleitung (Gl. 3.7), und der Strahlungsfluß ist proportional dem lokalen Temperaturgradienten. Trifft keiner dieser Grenzfälle zu, muß der Energietransport im Medium durch explizites Lösen der Strahlungstransportgleichung beschrieben werden (Mihalas 1978):

$$
\left[\frac{1}{c} \frac{\partial}{\partial t}+(\underline{n} \cdot \underline{\partial})\right] I_{\nu}(\underline{n}, \underline{r}, t)=j_{\nu}(\underline{n}, \underline{r}, t)-\kappa_{\nu}(\underline{n}, \underline{r}, t) I_{\nu}(\underline{n}, \underline{r}, t) .
$$

Hierin ist $\underline{n}$ der Einheitsvektor in Ausbreitungsrichtung der Strahlung am Ort $\underline{r}$ der betrachteten Region, $I_{\nu}$ die Intensität (in $\mathrm{erg} \mathrm{s}^{-1} \mathrm{~cm}^{-2} \mathrm{~Hz}^{-1} \mathrm{sr}^{-1}$ ) bei der Frequenz $\nu, j_{\nu}$ die Emissivität (in $\operatorname{erg~s}^{-1} \mathrm{~cm}^{-3} \mathrm{~Hz}^{-1} \mathrm{sr}^{-1}$ ) und $\kappa_{\nu}$ der Absorptionskoeffizient (in $\mathrm{cm}^{-1}$ ) des Mediums. Der Strahlungsfluß ist die über den Raumwinkel $\Omega$ (in sr) gemittelte, frequenzintegrierte Intensität in Richtung $\underline{n}$ :

$$
\underline{F}_{\mathrm{rad}}(\underline{r}, t)=\int_{\nu} \int_{\Omega} I_{\nu}(\underline{n}, \underline{r}, t) \underline{n} \mathrm{~d} \Omega \mathrm{d} \nu .
$$


Zur Berechnung des Strahlungsflusses ist also die Kenntnis von $I_{\nu}(\underline{n}, \underline{r}, t)$ nötig. Die Lösung der Strahlungstransportgleichung erfordert aber die Angabe der Emissivität $j_{\nu}$ und des Absorptionskoeffizienten $\kappa_{\nu}$, die wiederum Funktionen der Dichte und Temperatur der Region sind. Die Energiegleichung der Elektronen (Gl. 3.4) sowie die restlichen hydrodynamischen Gleichungen und die Strahlungstransportgleichung (Gl. 3.8), bzw. das Integral ihrer Lösung (Gl. 3.9), sind daher in einer nichttrivialen Art gekoppelt und können nur numerisch gelöst werden.

Während die Berücksichtigung der Viskosität und der Wärmeleitung die Ordnung des Differentialgleichungssystems um eins erhöht, führt die allgemeine Form für einen Strahlungsverlust des Plasmas auf ein System aus Differentialgleichungen und einer Integralgleichung, also auf eine „Integro-Differentialgleichung“. Damit ist eine einfache Lösung mit Methoden des Differentialgleichungskalküls nicht mehr möglich, und aufwendigere numerische Verfahren sind anzuwenden. Um den erforderlichen Aufwand zu begrenzen, sollen in den folgenden Abschnitten Näherungen durchgeführt werden, die das obige Gleichungssystem auf die wesentlichen Prozesse in den zu untersuchenden AM Her-Systemen beschränken.

\subsection{Annahmen und Näherungen}

\subsubsection{Stationarität und eindimensionale Strömung}

Beobachtungdaten einiger AM Her-Systeme zeigen neben der phasenabhängigen Intensitätsvariation durch die Akkretionsgeometrie noch kurzperiodische Schwankungen mit kleiner Amplitude (Abb. 2.4). Ein Erklärungsmodell dazu geht von einer zeitabhängigen Veränderung der Akkretionsrate des Systems aus, setzt aber einen stabilen hydrodynamische Stoß über der Sternoberfläche voraus (Wolff, Wood \& Imamura 1991; Wood, Imamura \& Wolff 1992; Wu, Chanmugam \& Shaviv 1992; Steiman-Cameron et al. 1994). Da die Strahlung aus der Emissionsregion von der Akkretionsrate abhängt, können durch geeignete, ad hoc angenommene Modulationen („Rauschen“) eines Mittelwerts Intensitätsschwankungen erzeugt werden, die den Beobachtungen entsprechen.

Ein anderes Modell betrachtet allgemein die Stabilität des Plasmas gegen Störungen der Gleichgewichtslage eines Stoßes bei konstanter Akkretionsrate (siehe Kap. 2.3). Ergebnisse liefern eine kurzperiodische Oszillation der Stoßhöhe, die eine den Beobachtungen ähnliche Intensitätsschwankung erzeugen können. Allerdings gehen hier wesentlich die intrinsischen Eigenschaften der Emissionsregion und der darin dominanten Kühlprozesse ein. Es können Fälle konstruiert werden in denen eine gedämpfte Oszillation auftritt oder die Amplitude zu groß ist, um die beobachteten Intensitäten zu beschreiben.

Die Modelle erklären die beobachteten Oszillationen übereinstimmend mit kleinen Veränderungen in der Stoßhöhe und der daraus resultierenden Strahlung der Emissionsregion. Da die Kühlprozesse einen wesentlichen Einfluß auf die Ergebnisse haben, bleibt die Erklärungsweise allerdings unbefriedigend und erfordert eine detailliertere Untersuchung des zeitlichen Verhaltens des Akkretionsplasmas. In dieser Arbeit wird darauf je- 
doch nicht eingegangen, so daß die Existenz einer stabilen, stationären Lösung für die Emissionsregion vorausgesetzt wird. Damit gilt in obigem Gleichungssystem: $\frac{\partial}{\partial t} \equiv 0$.

Die einfachste Annahme für die Magnetfeldgeometrie in Polaren ist die eines im Zentrum des weißen Zwergs entspringenden Dipolfelds. Spektropolarimetrische und spektroskopische Beobachtungen von einzelstehenden weißen Zwergen und Modellrechnungen zur Magnetfeldverteilung auf der Sternoberfläche weisen darauf hin, daß die Feldtopologie sehr viel komplizierter sein kann, doch lassen sich diese Konfigurationen im Rahmen der Modellgenauigkeiten mit einem aus dem Mittelpunkt verschobenem Dipolfeld erklären (siehe z. B. Burleigh, Jordan \& Schweizer 1999). Die Annahme eines Dipols ist zur grundlegenden Klärung der Verhältnisse in der Akkretionsregion hinreichend gut gerechtfertigt. Bei nicht zu hoch ausgedehnten Akkretionsregionen $\left(h_{\mathrm{sh}} \ll R_{\mathrm{wd}}\right)$ und Akkretion auf einen Bruchteil der Sternoberfläche $(f \ll 1)$ in der Umgebung des Pols, kann man weiterhin die Magnetfeldlinien des zentrierten Dipols als parallel und ungefähr senkrecht auf der Oberfläche des weißen Zwergs stehend betrachten. Die Einschränkung $h_{\mathrm{sh}} \ll R_{\mathrm{wd}}$ ermöglicht es außerdem, die Gravitationsbeschleunigung als konstant über die Emissionsregion hinweg anzunehmen: $g=g\left(R_{\mathrm{wd}}\right)$.

Der Sekundärstern ist in guter Näherung ein massearmer Hauptreihenstern, daher ist die Zusammensetzung des Akkretionsstroms in etwa solar (Massenanteil des Wasserstoffs: $X=0,7$, des Heliums: $Y=0,28$ und der schwereren Elemente: $Z=0,02)$. Bei den in Polaren vorherrschenden Leuchtkräften um $10^{33} \mathrm{erg} \mathrm{s}^{-1}$ und Temperaturen von einigen $10^{7} \mathrm{~K}$ ist der Wasserstoff- und Heliumanteil des Stroms bis in eine Entfernung vom weißen Zwerg, die den typischen Abstand der Komponenten übersteigt, weitgehend ionisiert (Kylafis \& Lamb 1982; Imamura et al. 1987). Da der Wasserstoff den größten Anteil ausmacht, ist es weiterhin gerechtfertigt die Ionen als Protonen anzusehen und den Akkretionsstrom als vollständig ionisiertes Wasserstoffplasma aufzufassen.

Die Elektronen und Protonen koppeln an das Magnetfeld und werden in die Polregion des Sterns geführt. Mit der Annahme der dort parallelen, senkrecht zur Oberfläche stehenden Feldlinien ist das Plasma in der Emissionsregion daher als eindimensionale Strömung konstanter Querschnittsfläche beschreibbar, und die Massenstromdichte $\dot{m}:=\dot{M} / A_{\text {acc }}$ ist unabhängig vom Ort, wenn die Akkretionsrate $\dot{M}$ konstant ist. Als Koordinate wird die geometrische Höhe $z$ über der Sternoberfläche gewählt. Der Differentialoperator des Gleichungssystems (Gln. 3.1 bis 3.4 und 3.8) vereinfacht sich in einem kartesischen Koordinatensystem damit zu: $\underline{\partial} \equiv\left(0,0, \frac{\mathrm{d}}{\mathrm{d} z}\right)$, und nur die $z$-Komponente der Vektoren ist ungleich null.

\subsubsection{Ideales Fluid}

Die dynamische Viskosität $\zeta$ und die Volumenviskosität $\xi$ stellen die Geschwindigkeitsänderung eines Fluidelements (bzw. des Plasmas) bei Scherung und Dehnung, d. h. unter der Wirkung von Schub- und Normalspannungen, dar. Eine genaue Formel für diese Größen anzugeben, in der die Abhängigkeiten von z. B. Temperatur und Dichte erfaßt sind, ist eine anspruchsvolle Aufgabe der statistischen Plasmaphysik. Bisher ist eine Angabe dieser Formel in voller Allgemeinheit noch nicht gelungen. Einfache Näherungen ergeben z. B. 
für die dynamische Viskosität $\zeta \sim T^{5 / 2}$ (Spitzer 1962, nach Braginskii 1958); genauere Zusammenhänge sind evtl. durch Experimente zu erlangen.

Die Viskosität in der Impulsgleichung (Gl. 3.2) erfaßt die Widerstandskraft, die die Teilchen durch Reibung erfahren. In der Energiegleichung der Ionen (Gl. 3.3) beschreibt der viskose Term den dissipativen Prozeß der Umwandlung von gerichteter Energie in Wärme; dagegen erfahren die Elektronen wegen ihrer geringen Masse keine Energieverluste durch Reibung. Dabei geht die Viskosität mit der Ortsableitung des Geschwindigkeitsgradienten ein und dominiert so in Bereichen starker Änderungen des Geschwindigkeitsfeldes im Fluid.

Bei der Akkretion auf den weißen Zwerg muß der freifallende Plasmastrom abgebremst werden, um auf der (als undurchdringlich angenommen) Sternoberfläche zur Ruhe zu kommen. In diesem Bremsgebiet ändert sich die Geschwindigkeit sehr stark, und durch die vorhandene Viskosität wird die beim Fall gewonnene Energie dissipiert; das Plasma wird aufgeheizt. Auf einer mikroskopischen Skala geschieht dieser Prozeß innerhalb einiger freier Weglängen der Ionen, da diese wegen ihrer hohen Masse die Energie liefern. Kann die umgesetzte Energie nicht rasch genug wieder abgegeben werden, bildet sich ein Übergangsgebiet („Stoß“), dessen Ausdehnung der freien Weglänge der Ionen entspricht und den freifallenden Strom vom geheizten Plasma trennt. In einer zeitlichen Entwicklung vergrößert sich der Abstand des Stoßes zur Sternoberfläche solange, bis die Kühlung (z. B. durch Strahlung) des Materials nach dem Stoß ausreicht, um am Ende der geheizten Region die Temperatur des weißen Zwergs zu erreichen. Die resultierende Höhe des Stoßes kann jedoch Oszillationen unterliegen (siehe Kap. 2.3).

Durch die Viskosität in den Gleichungen wird also die Ausbildung und Entwicklung eines hydrodynamischen Stoßes während der Akkretion beschrieben. Dabei bestimmt der numerische Wert dessen Ausdehnung, und in einer stationären geheizten Region (mit schwachem Einfluß) dessen Höhe über dem Stern. Die Werte der physikalischen Größen nach einem starken Stoß, wie z. B. die Rankine-Hugoniot-Temperatur (Gl. 1.7), sind allerdings unabhängig von der Viskosität.

Da die Kühlungsprozesse im Plasma die sich zwischen einem stabilen Stoß und der Sternoberfläche ausbildende Schichtung der Größen bestimmt, ist der Einfluß der Viskosität auf die Emissionsregion unwesentlich. In den Abbildungen 3.1 und 3.2 (folgende Seiten) sind die Temperaturverläufe der Ionen und Elektronen ( $T_{\mathrm{sh}}$ nach Gl. 1.7 mit $\mu=1$ ), sowie die Geschwindigkeit (positiv in Richtung auf den Stern zu) des Akkretionsstroms gegen die Schichtdicke für zwei Massenstromdichten eines stationären Stoßes aufgetragen. Verglichen werden die Ergebnisse aus eindimensionalen Rechnungen mit Viskosität und nach Vernachlässigung der viskosen Terme, bei unterschiedlich starkem Einfluß der Kühlungsprozesse (Bremsstrahlung und Zyklotronstrahlung). Bei Berücksichtigung der Viskosität wurde für diese ein mittlerer Wert gewählt, der für die angegebenen Parameter eine Ausdehnung des Stoßes von einigen freien Weglängen der Ionen erzeugt und in beiden Abbildungen konstant ist. Als Anfangswert der Temperatur wurde sowohl für Elektronen als auch für Ionen angenommen, daß dieser wesentlich unter der Stoßtemperatur liegt $\left(T_{\mathrm{i}} / T_{\mathrm{sh}}, T_{\mathrm{e}} / T_{\mathrm{sh}} \ll 1\right)$. Die Geschwindigkeit des Stroms am Anfang ist die Frei-Fall-Geschwindigkeit in der Nähe der Sternoberfläche $\left(v / v_{\mathrm{ff}}\left(R_{\mathrm{wd}}\right)=1\right)$. Der rasche 


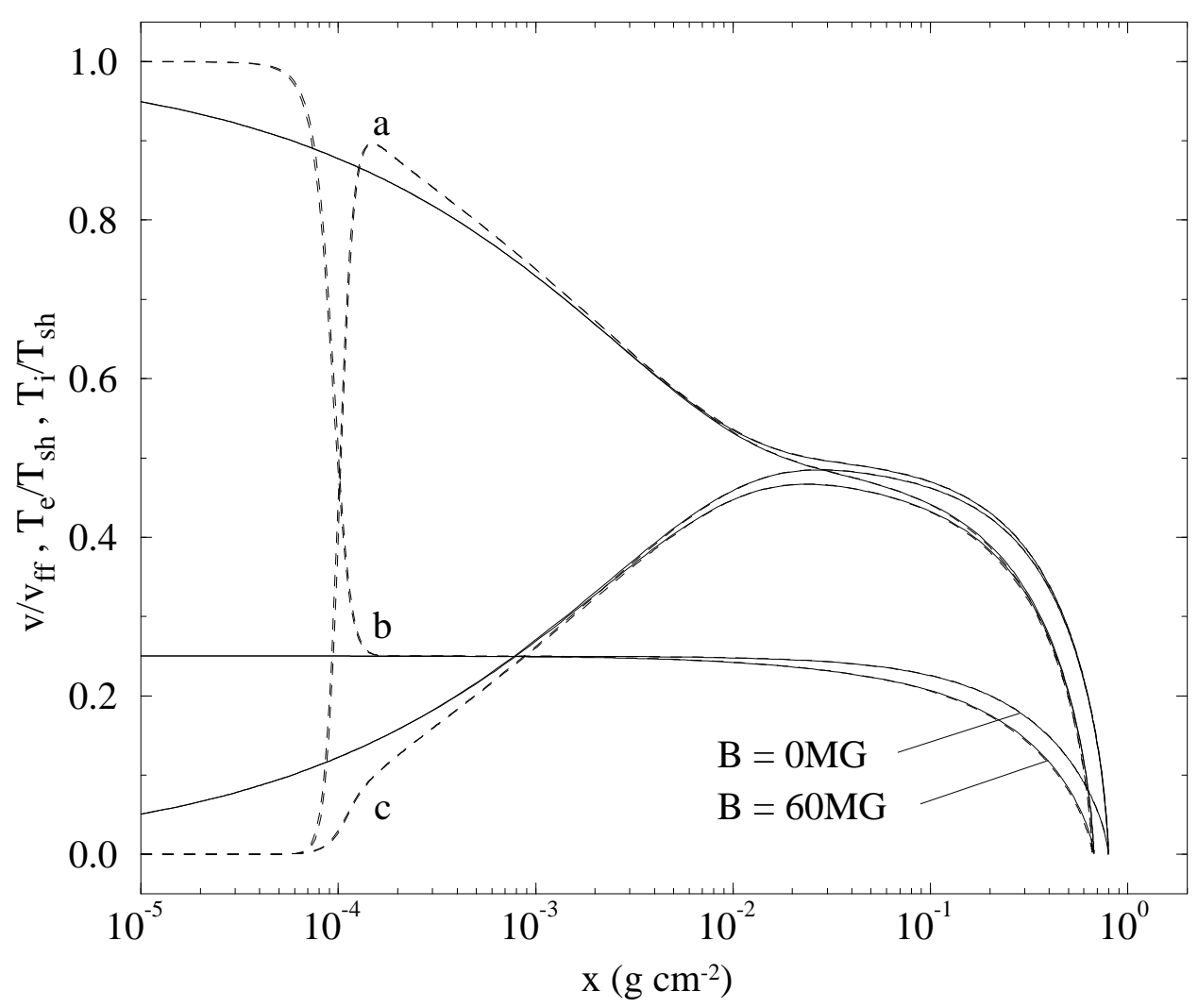

Abbildung 3.1: Temperatur- und Geschwindigkeitsverlauf in der Akkretionsregion unter Berücksichtigung (gestrichelte Linien) bzw. Vernachlässigung der Viskosität (durchgezogene Linien), für einen weißen Zwerg mit $M_{\mathrm{wd}}=0,6 M_{\odot}$ und $\dot{m}=100 \mathrm{~g} \mathrm{~s}^{-1} \mathrm{~cm}^{-2}$. Dargestellt sind normierte Ionen- (a) und Elektronentemperatur (c), sowie die normierte Geschwindigkeit (b) gegen die Schichtdicke, für den Fall reiner Bremsstrahlungskühlung $(B=0 \mathrm{MG})$ und zusätzlicher Zyklotronverluste in einem Magnetfeld mit $B=60 \mathrm{MG}$.

Anstieg der Ionentemperatur und das Absinken der Geschwindigkeit auf $v_{\mathrm{ff}} / 4$ kennzeichnen den Ort des starken Stoßes. In den Rechnungen ohne Viskosität wurde angenommen, daß die Schicht mit den Stoßwerten der physikalischen Größen beginnt. Die Ionen nehmen die Rankine-Hugoniot-Temperatur an $\left(T_{\mathrm{i}} / T_{\mathrm{sh}}=1\right)$, die Elektronen besitzen dagegen eine wesentlich kleinere Temperatur $\left(T_{\mathrm{e}} / T_{\mathrm{sh}} \ll 1\right)$. Der Anfangswert der Geschwindigkeit ist $v / v_{\mathrm{ff}}=1 / 4$.

Für große Massenstromdichten (Abb. 3.1) unterscheiden sich die Schichtungen nur am Anfang. Hier dominiert die Viskosität und erzeugt den starken Verdichtungsstoß in dem ein Teil der Ionenenergie in Wärme umgewandelt wird und so deren raschen Temperaturanstieg verursacht. Dabei erreichen die Ionen im Maximum nicht die Rankine-HugoniotTemperatur, da auch die Elektronen eine geringe Temperatur annehmen, denn wegen der starken elektrischen Wechselwirkung der Elektronen mit den Ionen wird auch das Elektronengas komprimiert. Für hohe $\dot{m}$ ist Bremsstrahlung der dominante Kühlprozeß, diese wirkt aber wesentlich bei hohen Dichten am Ende der geheizten Schicht, so daß die Elek- 


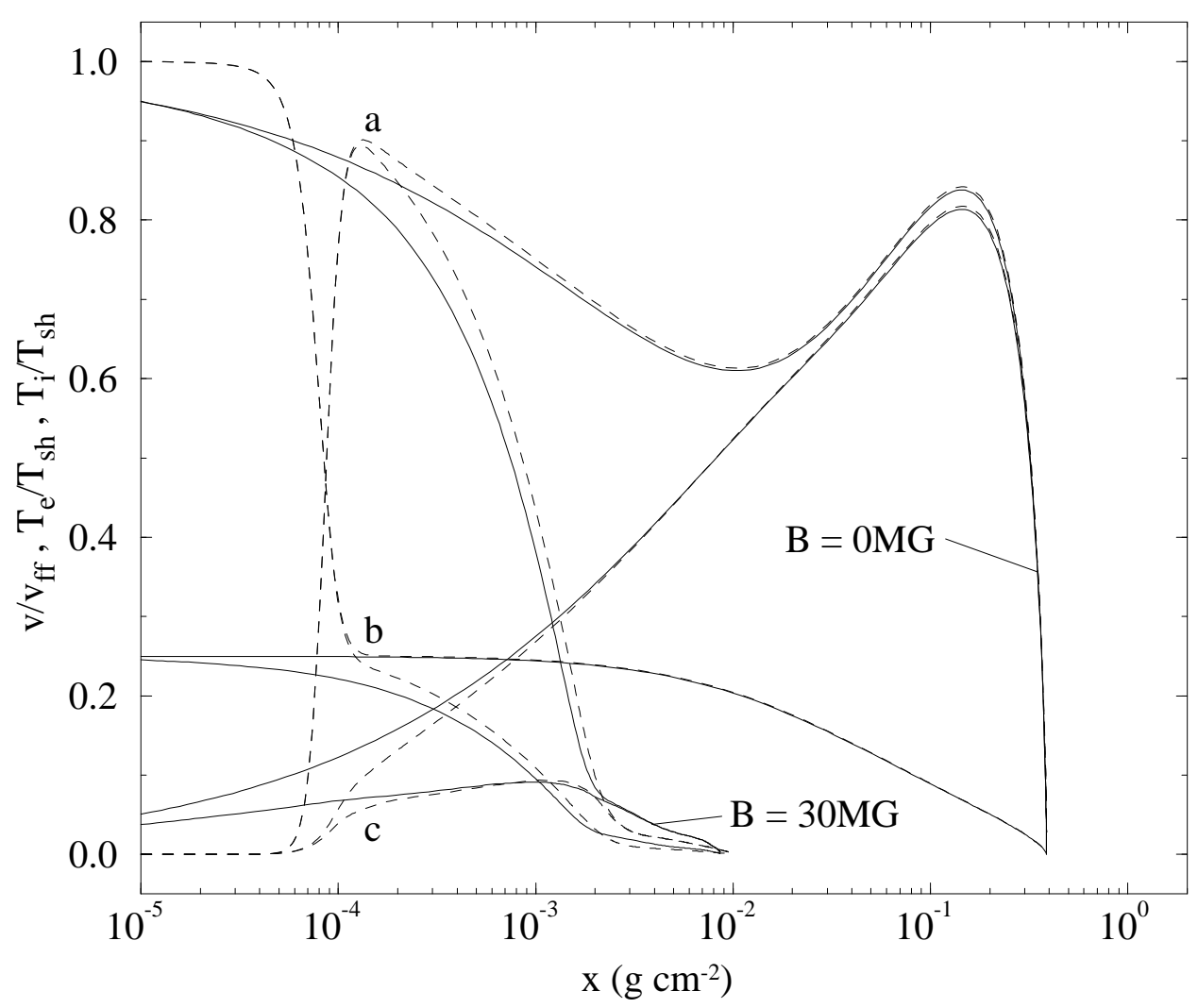

Abbildung 3.2: Darstellung der Temperatur- und Geschwindigkeitsverläufe wie in Abbildung 3.1, aber für $\dot{m}=10^{-2} \mathrm{~g} \mathrm{~s}^{-1} \mathrm{~cm}^{-2}$ und $B=30 \mathrm{MG}$.

tronen im Stoß kaum Strahlungsverluste erleiden und der Vorgang daher adiabatisch ist; die Elektronentemperatur wird um einen Faktor $\approx 2,5$ (für ein Wasserstoffplasma) erhöht (Zel'dovich \& Raizer 1966). (In der Abbildung ist der Faktor größer, da der numerische Wert der Viskosität in die Rechnungen eingeht.) Die Ergebnisse der Rechnungen ohne die viskosen Terme zeigen dagegen vom Beginn an einen Temperaturangleich von heißen Ionen und kälteren Elektronen durch die Coulomb-Wechselwirkung, wie er auch unmittelbar nach dem Stoß zu sehen ist. In diesem (geometrisch schmalen) Anfangsbereich spielt eine unterschiedlich starke Kühlung keine Rolle, die Ergebnisse aus den beiden Rechnungen (reine Bremsstrahlung bzw. Bremsstrahlung und Zyklotronstrahlung) sind nahezu identisch. Haben die Elektronen eine hinreichend hohe Temperatur angenommen und ist die Dichte ausreichend groß, werden die Strahlungsprozesse effizient („Emissionsregion“). Die Kurven der physikalischen Größen unterscheiden sich jetzt bezüglich der betrachteten Kühlungsprozesse, und die stärkere Kühlung (Bremsstrahlung und Zyklotronstrahlung zu $B=60 \mathrm{MG}$ ) ergibt eine insgesamt geringer ausgedehnte Akkretionsregion. Hinsichtlich der Unterscheidung nach der Viskosität zeigen die Verläufe jedoch kaum Abweichungen: In der Emissionsregion ist die Viskosität zu vernachlässigen.

Abbildung 3.2 zeigt die Ergebnisse der entsprechenden Rechnungen für $\dot{m}=$ $10^{-2} \mathrm{~g} \mathrm{~s}^{-1} \mathrm{~cm}^{-2}$. Bei nur schwacher Kühlung durch Bremsstrahlung $(B=0 \mathrm{MG})$ sind 
die Verläufe von Temperatur und Geschwindigkeit bezüglich der Viskosität ähnlich denen bei hoher Massenstromdichte: Die Viskosität bestimmt den Stoß, ist aber in der Emissionregion vernachlässigbar. Bei zusätzlicher Zyklotronstrahlung $(B=30 \mathrm{MG})$ - und somit erhöhter Kühlung des Plasmas - treten allerdings Abweichungen auf. Hier sind die Resultate, unabhängig von der Einbeziehung der Viskosität, zwar in qualitativer Übereinstimmung, insbesondere ist die Ausdehnung der geheizten Schichten nahezu gleich und die Elektronentemperaturen erreichen bei ungefähr der gleichen Schichtdicke ihr Maximum. Doch weichen z. B. die Geschwindigkeiten schon kurz nach dem Stoß voneinander ab, und die viskosen Terme sorgen für eine raschere Abbremsung des Stroms am Ende der geheizten Schicht. Damit liegt auch die Temperatur der Elektronen in diesem Bereich unter der für den Fall vernachlässigter Viskosität. Ein Vergleich der Stoßausdehnung und der Ausdehnung der Emissionsregion ergibt ein Verhältnis 1:5. Somit ist die Ausdehnung der Kühlregion von der gleichen Größenordnung wie die freie Weglänge der Ionen. Bei dieser Kombination von kleiner Massenstromdichte und starker Kühlung liegen daher eher die Verhältnisse der Teilchenheizung vor (siehe Kap. 1.2.4). Der Zugang eines viskosen Fluids ist also ungeeignet; die entsprechenden Verläufe der physikalischen Größen sind nicht verläßlich. Stattdessen müssen die Ergebnisse aus der Rechnung nach Vernachlässigung der viskosen Terme mit Schichtungen aus den Rechnungen der Teilchenheizung verglichen werden.

Unter der Annahme der Existenz einer stationären Lösung mit einem stabilen Stoß (siehe Kap. 3.2.1) ist es also innerhalb des Gültigkeitsbereichs der hydrodynamischen Beschreibung gerechtfertigt, die Viskosität zu vernachlässigen und das Plasma als ideales Fluid aufzufassen: $\zeta \equiv 0$ und $\xi \equiv 0$. Damit fallen die Terme des Gleichungssystems in denen $\zeta$ und $\xi$ stehen fort, und der Beginn der geheizten Region wird mit den Werten der physikalischen Größen nach einem starken Stoß (Gln. 1.5 bis 1.7) definiert.

\subsubsection{Einfluß der Wärmeleitung}

Wärmeleitung beschreibt den Energietransport, der durch ein Temperaturgefälle in einem Medium entsteht. Dabei wird durch wechselseitige Stöße der Teilchen des Materials Energie in Gegenrichtung des Temperaturgradienten fließen ohne einen Teilchentransport zu verursachen. Daher kann in einem ionisierten Plasma i. allg. der Beitrag der Ionen zum Energiefluß (wegen ihrer größeren Masse) gegen den der Elektronen vernachlässigt werden. Befinden sich in dem Medium keine Energiequellen oder -senken, wird der Temperaturunterschied in einer zeitlichen Entwicklung ausgeglichen, und als stationärer Zustand bildet sich eine mittlere Temperatur aus; die Wärmeleitung kommt zum erliegen.

Im Fall eines hydrodynamischen Stoßes wird Wärmeleitung in den Bereichen mit großen Temperaturgradienten dominant sein: am Beginn der geheizten Schicht und im Bereich des Übergangsgebiets zum Stern. Es konnte jedoch gezeigt werden (Kuijpers \& Pringle 1982; Frank, King \& Lasota 1983), daß nur eine Lösung für die geheizte Region zutreffend ist, in der der Energiefluß durch Wärmeleitung beim Anschluß an den Stern gegen null geht. Im Bereich des Anstiegs der Elektronentemperatur nach dem Stoß bewirkt Wärmeleitung einen schnelleren Temperaturausgleich mit den Ionen durch Verringerung 
des Gradienten. Damit wird die Temperatur der Elektronen im Stoß ebenfalls erhöht, und wegen ihrer geringen Masse kann deren thermische Geschwindigkeit ausreichen, um in das Gebiet vor den Stoß zu gelangen (Zel'dovich \& Raizer 1966; Imamura et al. 1987). Dort heizen sie das Plasma auf, und auch die Ionen erreichen eine höhere Temperatur als im Fall der vernachlässigten Wärmeleitung. Es bildet sich der sog. „precursor", in dem die Elektronen eine größere Temperatur als die Ionen besitzen (Abb. 1.5). Die Elektronen ändern somit kaum ihre Temperatur über den Stoß hinweg, während die Ionentemperatur innerhalb einer freien Weglänge der Ionen stark ansteigt.

Die Auswirkungen der Wärmeleitung auf die Temperaturstruktur der geheizten Schicht - im Vergleich zu den Resultaten bei deren Vernachlässigung - bestehen damit zum einen in einer globalen Absenkung der Ionentemperatur. Zum anderen wird die Temperatur der Elektronen im Bereich vor dem Stoß (mit geringer Massendichte des Stroms) deutlich angehoben. Weiterhin geschieht der Angleich an die Temperatur der Ionen rascher, was in einer Verschiebung des Maximalwerts der Elektronentemperatur in Richtung des Ortes des Stoßes resultiert.

Die veränderte Temperaturschichtung hat nur unwesentliche Auswirkungen auf die Emission der geheizten Region, da der Hauptteil der Strahlung der Elektronen aus den Gebieten hoher Temperatur bzw. großer Dichte stammt und diese Bereiche kaum beeinflußt werden. Allerdings wirkt der Elektronen-precursor bei paralleler Sicht zur Flächennormale der Akkretionsregion als absorbierendes, ionisiertes Medium geringer Dichte für die Strahlung der Emissionsregion und erzeugt somit atomare Absorptionslinien entsprechend der Zusammensetzung des Akkretionsstroms.

Darüberhinaus zeigt eine Abschätzung der Energieflüsse durch Strahlung und Wärmeleitung, daß bei den typischen Parametern der AM Her-Systeme Wärmeleitung einen vernachlässigbaren Anteil am Gesamtenergiefluß hat. Mit der Abschätzung des Strahlungsflusses durch Zyklotronstrahlung $\underline{F}_{\text {cyc }}(\mathrm{Gl} .2 .8)$ und dem Energiefluß durch Wärmeleitung $\underline{Q}$ (Gl. 3.7) dominiert Wärmeleitung, wenn gilt:

$$
\left(\frac{B}{10^{7} \mathrm{G}}\right)^{2,85}\left(\frac{\dot{m}}{1 \frac{\mathrm{g}}{\mathrm{cm}^{2} \mathrm{~s}}}\right)^{0,15} \leq 2,35 \cdot 10^{-3}\left(\frac{M_{\mathrm{wd}}}{M_{\odot}} \frac{R_{\odot}}{R_{\mathrm{wd}}}\right)^{1,075} .
$$

Erst bei sehr kleinen Massenflüssen $\dot{m}<10^{-3} \mathrm{~g} \mathrm{~cm}^{-2} \mathrm{~s}^{-1}$ und Magnetfeldstärken $B<$ $10 \mathrm{MG}$ bzw. hohen Massen des weißen Zwergs $M_{\mathrm{wd}}>1,0 M_{\odot}$ sind die Strahlungsflüsse so gering, daß die Wärmeleitung effektiv wird.

Damit ist der Einfluß der Wärmeleitung in der Akkretionsregion der Polare - im Vergleich zu den auftretenden Strahlungsflüssen - von sekundärer Bedeutung, und die Vernachlässigung des entsprechenden Terms in der Energiegleichung der Elektronen (Gl. 3.4) ist gerechtfertigt; es gilt: $\underline{Q} \equiv 0$.

\subsubsection{Behandlung der Streuung im Strahlungstransport}

Bei den typischen Parametern für die Masse des weißen Zwergs und die Massenstromdichte der Polare sind die Temperaturen in der Akkretionsregion so hoch, daß die Materie 
zum größten Teil als vollständig ionisiert angenommen werden kann (siehe Kap. 3.2.1). Die Strahlung der Emissionsregion wechselwirkt daher mit einer großen Anzahl an freien Elektronen und Ionen (resp. Protonen). Der wesentliche Prozeß ist dabei die Streuung der Photonen an den freien Elektronen, da die Ionen wegen ihrer hohen Masse kaum als Streuzentren auftreten.

Ist die Photonenenergie größer als die thermische Energie der Elektronen $\left(h \nu \gg k_{\mathrm{B}} T_{\mathrm{e}}\right.$; $h$ : Planckkonstante), verlieren die Photonen bei dem Streuvorgang einen Teil ihrer Energie an die Elektronen („Compton-Streuung“). Bei diesem Prozeß erhöht sich die Temperatur der Elektronen, während die Strahlung zu kleineren Frequenzen hin verschoben wird. Im Fall der Akkretionsregion trifft dies auf die Bereiche vor dem Stoß und in der Nähe der Sternoberfläche zu. Hier wechselwirkt die intensive harte Röntgenstrahlung mit den kühlen Elektronen und erhöht die Temperatur in diesen Gebieten, wobei die Photonen in den Spektralbereich der weichen Röntgenstrahlung bzw. in das UV gestreut werden.

Der entgegengesetzte Prozeß (,inverse Compton-Streuung“) tritt auf, wenn die Energie der Strahlung kleiner als die der Elektronen ist $\left(h \nu \ll k_{\mathrm{B}} T_{\mathrm{e}}\right)$. Nun transferieren die Elektronen einen Teil ihrer Energie auf die Photonen, deren Frequenz erhöht wird. Dagegen führt der Energieverlust der Elektronen zu einer Absenkung ihrer Temperatur; der Prozeß wirkt somit als Kühlungsmechanismus für das Elektronengas. In der Emissionsregion der Polare trifft diese Situation auf die Bereiche hoher Temperatur zu; im Modell der Stoßheizung auf das Gebiet kurz hinter dem Stoß. Die Elektronentemperatur ist hier so hoch, daß eine Wechselwirkung mit der UV- bzw. weichen Röntgenstrahlung (z. B. dem reprozessierten Anteil aus der Atmosphäre des weißen Zwergs) stattfindet. Die Elektronen werden dabei abgekühlt und die Strahlung zu größeren Frequenzen in den Spektralbereich der harten Röntgenstrahlung gestreut.

Die Effizienz des Energieübertrags in diesen Streuprozessen hängt von der Intensität des umgebenen Strahlungsfeldes und der Anzahl der vorhandenen Elektronen ab. Der Energiegewinn der Elektronen, $\Lambda_{\mathrm{C}}$ (in $\mathrm{erg} \mathrm{s}^{-1} \mathrm{~cm}^{-3}$ ), bei der Compton-Streuung bzw. Energieverlust bei dem inversen Prozeß ist (Kylafis \& Lamb 1979, nach Levich \& Sunyaev 1971 bzw. Weymann 1965)

$$
\Lambda_{\mathrm{C}} \sim \sigma_{\mathrm{T}} c n_{\mathrm{e}} U \frac{k_{\mathrm{B}} T}{m_{\mathrm{e}} c^{2}},
$$

mit $T=T_{\nu}$ der Temperatur der Photonen $\left(k_{\mathrm{B}} T_{\nu} \equiv h \nu\right)$ im Fall der Compton-Streuung bzw. $T=T_{\mathrm{e}}$ der Temperatur der Elektronen für inverse Compton-Streuung. Dabei wurde angenommen, daß die Energie der Photonen kleiner als die Ruheenergie der Elektronen ist $\left(h \nu \ll m_{\mathrm{e}} c^{2}\right)$, so daß der Thomson-Wirkungsquerschnitt $\sigma_{\mathrm{T}}$ benutzt werden kann, ohne die Korrekturen der Quanten-Elektrodynamik in Form des Klein-NishinaWirkungsquerschnitts zu berücksichtigen. Weiterhin ist $U$ die Energiedichte (in $\mathrm{erg} \mathrm{cm}^{-3}$ ) des umgebenen Strahlungsfeldes:

$$
U:=\frac{1}{c} \int_{\nu} \int_{\Omega} I_{\nu}(\underline{n}, \underline{r}, t) \mathrm{d} \Omega \mathrm{d} \nu .
$$

Für eine Abschätzung des Einflusses der Streuvorgänge auf die Struktur der Emissionsregion kann man zunächst annehmen, daß die Energiedichte des Strahlungsfeldes 
durch einen Bruchteil der Akkretionsleuchtkraft (Gl. 1.1) erzeugt wird. Damit gilt für den Energieübertrag:

$$
\Lambda_{\mathrm{C}} \sim n_{\mathrm{e}} T U \sim n_{\mathrm{e}} T \frac{M_{\mathrm{wd}}}{R_{\mathrm{wd}}} \dot{M} .
$$

Die Streuung wird daher bei großen Massen des weißen Zwergs und hohen Akkretionsraten einflußreich werden.

Detaillierte numerische Berechnungen einer sphärischen Akkretionsgeometrie unter Einbeziehung der Streuprozesse (Kylafis \& Lamb 1982; Imamura et al. 1987) bestätigen diese Tendenz. Der Effekt der Heizung der kühlen Gebiete durch die ComptonStreuung tritt effektiv nur im Bereich um $M_{\text {wd }} \approx 1,0 M_{\odot}$ und $\dot{M} \geq 0,1 \dot{M}_{\text {Edd }}$ auf, wobei die transferierte Energie immer unterhalb 10\% der Akkretionsleuchtkraft bleibt. Die Kühlung des Elektronengases durch die inverse Compton-Streuung erreicht zwar Werte, die über $10 \% L_{\text {acc }}$ liegen, aber ebenfalls nur in einem relativ begrenzten Parameterbereich $\left(M_{\text {wd }}>1,0 M_{\odot}, \dot{M} \geq 0,01 \dot{M}_{\text {Edd }}\right)$. Diese Ergebnisse werden zudem noch durch den Einfluß der Geometrie der Akkretionsregion beeinflußt. Nimmt man statt der sphärisch symmetrischen Akkretion ein durch Magnetfelder begrenztes, ebenes Akkretionsgebiet über einen Bruchteil der Oberfläche des weißen Zwergs an, können eine Vielzahl der Photonen die Emissionsregion ohne Wechselwirkung (oder nach wenigen Stößen) verlassen. Die Auswirkungen durch die Streuung werden somit vermindert.

Die Effekte durch Compton-Streuung sind daher von nachrangiger Bedeutung im Vergleich z. B. zum Strahlungstransport in der Emissionsregion der Polare, deren typischer Wert für die Masse des weißen Zwergs bei 0,6 $M_{\odot}$ liegt. Eine detaillierte Einbeziehung dieser Prozesse in ein Akkretionsmodell wird in dieser Arbeit nicht vorgenommen.

Während die Auswirkungen der Compton-Streuung auf die Struktur der Emissionsregion vernachlässigt werden können, sollen die Effekte der kohärenten (elastischen) Elektronenstreuung („Thomson-Streuung“) berücksichtigt werden. Hierbei geschieht kein Energieübertrag zwischen Photonen und Elektronen. Stattdessen werden Photonen aus ihrer Richtung abgelenkt (entsprechend einem absorptiven Prozeß) und in andere Bereiche der Emissionsregion befördert (entsprechend einer Erhöhung der lokalen Emission), wobei weiterhin näherungsweise angenommen werden kann, daß die Streuung isotrop ist. Damit wird die Strahlungstransportgleichung (Gl. 3.8) verändert zu:

$$
\left[\frac{1}{c} \frac{\partial}{\partial t}+(\underline{n} \cdot \underline{\partial})\right] I_{\nu}(\underline{n}, \underline{r}, t)=j_{\nu}(\underline{n}, \underline{r}, t)+j_{\nu}^{\mathrm{s}}(\underline{n}, \underline{r}, t)-\left(\kappa_{\nu}(\underline{n}, \underline{r}, t)+\sigma^{\mathrm{s}}(\underline{r}, t)\right) I_{\nu}(\underline{n}, \underline{r}, t) .
$$

Darin ist $\sigma^{\mathrm{s}}:=n_{\mathrm{e}} \sigma_{\mathrm{T}}$ der Streukoeffizient der kohärenten Thomson-Streuung unter der Annahme, daß die Quanteneffekte vernachlässigbar sind $\left(h \nu \ll m_{\mathrm{e}} c^{2}\right)$. Er erhöht den Absorptionskoeffizienten des Materials und beschreibt damit die Abnahme der Intensität durch Streuung von Photonen aus der Richtung $\underline{n}$. Die Veränderung der Emissivität des Materials wird durch den Term $j_{\nu}^{\mathrm{s}}$ beschrieben:

$$
j_{\nu}^{\mathrm{s}}(\underline{n}, \underline{r}, t):=\sigma^{\mathrm{s}}(\underline{r}, t) \frac{1}{4 \pi} \int_{\Omega} I_{\nu}(\underline{n}, \underline{r}, t) \mathrm{d} \Omega .
$$


Die über den Raumwinkel $\Omega$ gemittelte Intensität berücksichtigt dabei den Gewinn von Photonen aus allen Richtungen des Strahlungsfeldes, während die Proportionalität zum Streukoeffizienten die Anzahl der Elektronen, die eine Streuung verursachen, erfaßt.

Durch die Einbeziehung der Streuung verändert die Strahlungstransportgleichung ihren Charakter und wird zu einer Integro-Differentialgleichung, für die in der Literatur ausgearbeitete Lösungsmethoden existieren (Mihalas 1978).

\subsection{Betrachtetes Gleichungssystem und Lösungsme- thode}

Bei Berücksichtigung der obigen Annahmen bzw. Näherungen und nach Einführung der Geschwindigkeitsvariable $w=-v$ in Richtung der (als konstant angenommenen) Gravitationsbeschleunigung $g=\left(0,0,-g\left(R_{\mathrm{wd}}\right)\right)$, vereinfacht sich das hydrodynamische Gleichungssystem (Gln. 3.1 bis 3.4) zu:

- Kontinuitätsgleichung:

$$
\rho w=\dot{m}
$$

- Euler-Gleichung:

$$
w \frac{\mathrm{d} w}{\mathrm{~d} z}+\frac{1}{\rho} \frac{\mathrm{d}}{\mathrm{d} z}\left(P_{\mathrm{e}}+P_{\mathrm{i}}\right)=-g
$$

- Energiegleichung der Ionen bzw. Elektronen:

$$
\begin{aligned}
& -w \frac{\mathrm{d}}{\mathrm{d} z}\left(\rho u_{\mathrm{i}}\right)+\frac{w}{\rho}\left(P_{\mathrm{i}}+\rho u_{\mathrm{i}}\right) \frac{\mathrm{d} \rho}{\mathrm{d} z}=-\Lambda_{\mathrm{x}}, \\
& -w \frac{\mathrm{d}}{\mathrm{d} z}\left(\rho u_{\mathrm{e}}\right)+\frac{w}{\rho}\left(P_{\mathrm{e}}+\rho u_{\mathrm{e}}\right) \frac{\mathrm{d} \rho}{\mathrm{d} z}=\Lambda_{\mathrm{x}}-\frac{\mathrm{d} F_{\mathrm{rad}}}{\mathrm{d} z} .
\end{aligned}
$$

Geht man weiterhin zur Schichtdicke $x$ als unabhängige Variable über $(\mathrm{d} x:=-\rho \mathrm{d} z)$, führt folgende normierte, dimensionslose Größen ein

$$
\begin{aligned}
v & :=\frac{w}{v_{\mathrm{ff}}\left(R_{\mathrm{wd}}\right)} & \gamma & :=\frac{g}{\dot{m} v_{\mathrm{ff}}\left(R_{\mathrm{wd}}\right)} \\
\Pi_{\mathrm{i}} & :=\frac{P_{\mathrm{i}}}{\dot{m} v_{\mathrm{ff}}\left(R_{\mathrm{wd}}\right)} & \lambda_{\mathrm{x}} & :=\frac{\Lambda_{\mathrm{x}}}{\dot{m}^{2} v_{\mathrm{ff}}\left(R_{\mathrm{wd}}\right)} \\
\Pi_{\mathrm{e}} & :=\frac{P_{\mathrm{e}}}{\dot{m} v_{\mathrm{ff}}\left(R_{\mathrm{wd}}\right)} & \lambda_{\mathrm{rad}} & :=\frac{\Lambda_{\mathrm{rad}}}{\dot{m}^{2} v_{\mathrm{ff}}\left(R_{\mathrm{wd}}\right)} \quad \text { mit } \quad \Lambda_{\mathrm{rad}}:=-\rho \frac{\mathrm{d} F_{\mathrm{rad}}}{\mathrm{d} x}
\end{aligned}
$$


und nutzt die Kontinuitätsgleichung (Gl. 3.16) aus, erhält man das zu lösende hydrodynamische Gleichungssystem:

$$
\begin{aligned}
\frac{\mathrm{d} v}{\mathrm{~d} x} & =\frac{\gamma+\frac{2}{3} \lambda_{\mathrm{rad}}}{1-\frac{5}{3} \frac{\Pi_{\mathrm{i}}+\Pi_{\mathrm{e}}}{v}} \\
\frac{\mathrm{d} \Pi_{\mathrm{i}}}{\mathrm{d} x} & =\frac{2}{3}\left(-\lambda_{\mathrm{x}}-\frac{5}{2} \frac{\Pi_{\mathrm{i}}}{v} \frac{\mathrm{d} v}{\mathrm{~d} x}\right) \\
\frac{\mathrm{d} \Pi_{\mathrm{e}}}{\mathrm{d} x} & =\frac{2}{3}\left(\lambda_{\mathrm{x}}-\lambda_{\mathrm{rad}}-\frac{5}{2} \frac{\Pi_{\mathrm{e}}}{v} \frac{\mathrm{d} v}{\mathrm{~d} x}\right),
\end{aligned}
$$

wobei verwendet wurde, daß für einatomige, ideale Gas gilt: $u=\frac{3}{2} \frac{P}{\rho}$. (Für die Form des Systems bei Berücksichtigung der Viskosität siehe Woelk \& Beuermann (1996).)

Die Annahmen und Näherungen für den Strahlungstransport (Gl. 3.14) führen zu folgender Form:

$$
-\rho \cos \vartheta \frac{\mathrm{d} I_{\nu}}{\mathrm{d} x}=\kappa_{\nu} B_{\nu}+j_{\nu}^{\mathrm{s}}-\left(\kappa_{\nu}+\sigma^{\mathrm{s}}\right) I_{\nu}
$$

Darin ist $\vartheta$ der Winkel zwischen Ausbreitungsrichtung der Strahlung und einer Vorzugsrichtung. Im folgenden soll dies die Richtung der Magnetfeldlinien in der Akkretionsregion sein, die als parallel zu deren Flächennormale verlaufend angenommen werden (siehe Kap. 3.2.1); $\vartheta=0^{\circ}$ entspricht somit senkrechter Sicht auf die Akkretionsfläche. Des weiteren geht hier der Kirchhoff-Satz für einen thermischen Strahler ein:

$$
\frac{j_{\nu}}{\kappa_{\nu}}=B_{\nu}=\frac{2 h}{c^{2}} \nu^{3}\left[e^{\frac{h \nu}{k_{\mathrm{B}} T}}-1\right]^{-1},
$$

mit der Kirchhoff-Planck-Funktion $B_{\nu}$. Dies ist gerechtfertigt, denn die Veränderungen der thermodynamischen Größen in der Emissionsregion sind über die mittlere freie Weglänge der Photonen gering, und ein Temperaturgleichgewicht stellt sich wenigstens lokal ein („lokales thermodynamisches Gleichgewicht“, LTE). Der Absorptionskoeffizient $\kappa_{\nu}:=\kappa_{\nu}^{\mathrm{ff}}+\kappa_{\nu}^{\mathrm{cyc}}$ ist die Addition der entsprechenden Koeffizienten für Bremsstrahlung und Zyklotronstrahlung, da nur diese Emissionsprozesse im folgenden betrachtet werden sollen. Die Form für den Absorptionskoeffizienten der Bremsstrahlung $\kappa_{\nu}^{\mathrm{ff}}$ findet sich in Rybicki \& Lightman (1979). Im Fall des Zyklotronabsorptionskoeffizienten $\kappa_{\nu}^{\text {cyc }}$ läßt sich i. allg. keine solche geschlossene Form angeben, da zu seiner Berechnung u. a. Integrale über Bessel-Funktionen in der Geschwindigkeitsverteilung der Elektronen auszuführen sind. Für eine genaue Darstellung verweise ich auf die Literatur (z. B. Bekefi 1966; Ramaty 1969). In dieser Arbeit wird für $\kappa_{\nu}^{\text {cyc }}$ eine numerische Berechnungsweise nach Chanmugam \& Dulk (1981) bzw. Thompson \& Cawthorne (1987) gewählt, die in ähnlicher Form auch in Woelk (1991) und Woelk \& Beuermann (1992; 1996) umgesetzt wurde. (Für einen Vergleich der in der Literatur verwendeten unterschiedlichen Zugangsweisen siehe z.B. Chanmugam et al. (1989).)

Zur Berechnung der Struktur der Emissionsregion ist das hydrodynamische Gleichungssystem (Gln. 3.20 bis 3.22) zusammen mit der Strahlungstransportgleichung (Gl. 3.23) mit folgenden Randbedingungen zu lösen: 
- oberer Rand der Akkretionsregion (hydrodynamischer Stoß bei $x \ll 1$ ):

$$
\begin{aligned}
v & =\frac{1}{4} \\
\Pi_{\mathrm{i}} & =\frac{3}{4} \\
\Pi_{\mathrm{e}} & \ll 1 \\
I_{\nu} & =0 \quad\left(\text { für } \quad 90^{\circ}<\vartheta \leq 180^{\circ}\right),
\end{aligned}
$$

- unterer Rand der Akkretionsregion:

$$
\begin{aligned}
v & \ll 1 \\
\Pi_{\mathrm{i}} & \approx \Pi_{\mathrm{e}} \\
I_{\nu} & =0 \quad\left(\text { für } \quad 0^{\circ} \leq \vartheta<90^{\circ}\right) .
\end{aligned}
$$

Die Werte der hydrodynamischen Größen am Beginn der Akkretionsregion (oberer Rand) werden durch die Bedingungen für einen starken Stoß festgelegt (siehe Kap. 1.2.2). Dort entspricht der Randbedingung für die Intensität die Annahme, daß keine Strahlung von außen in die Region einfällt. Die Wahl des Wertes für die Anfangsschichtdicke beeinflußt die Struktur nicht, da diese Koordinate nicht explizit in die Gleichungen eingeht. Das Ende der geheizten Schicht wird durch den nahezu vollständigen Verlust der kinetischen Energie des Stroms $(v \ll 1)$ definiert; der gewählte Wert für die Geschwindigkeit beeinflußt die Schichtung jedoch nicht. Am unteren Rand müssen sich weiterhin die Temperaturen (bzw. Drücke) von Elektronen und Protonen angeglichen haben (Äquipartition der Teilchenenergien). Aus der Annahme, daß keine Strahlung vom weißen Zwerg in die Emissionsregion eindringt ergibt sich die Bedingung für die Intensität. Da die Ausdehnung der Emissionsregion von der Effizienz der Kühlungsmechanismen abhängt, resultiert der Wert der maximalen Schichtdicke aus den Rechnungen.

Das zu lösende Gleichungssystem besteht aus drei Differentialgleichungen erster Ordnung, in die der Gradient des Strahlungsflusses $F_{\text {rad }}$ eingeht. Diese Größe wird über eine Integration der Intensität $I_{\nu}$ bestimmt (Gl. 3.9), für die wiederum eine Differentialgleichung zu lösen ist (Gl. 3.23). Die Form der einzelnen Gleichungen verhindert allerdings eine einfache Kopplung zu einem gemeinsamen System; Hydrodynamik und Strahlungstransport können daher nur getrennt berechnet werden.

Zur Lösung des Systems der hydrodynamischen Gleichungen (Gln. 3.20 bis 3.22 ) wurde ein vorgefertigtes Programmpaket („SLGA2" von K. Raith, Rechenzentrum Karlsruhe) benutzt, das auf einem impliziten Prädiktor-Korrektor-Verfahren beruht und Differenzenformeln der Ordnung 1 bis 5 verwendet. Schrittweite und Ordnung werden dabei vom Programm gewählt, um den (geschätzten) lokalen Fehler einer Lösung unterhalb einer vorzugebenen Schranke zu halten. Die resultierenden, nichtlinearen Differenzengleichungen werden mit einem vereinfachten Newton-Verfahren iterativ gelöst, wobei als Startwert der Lösungsvektor der vorangehenden Stützstelle in der unabhängigen Variable verwendet wird. Eine ausführliche Darstellung findet man in der im Programmpaket enthaltenen Dokumentation und in Raith, Schönauer \& Glotz (1979). 
Der Strahlungstransport (Gl. 3.23) wird unter der Annahme des lokalen thermodynamischen Gleichgewichts (LTE) nach der von G. Rybicki (Mihalas 1978), zur Berechnung von Sternatmosphären, entworfenen Methode gelöst. Dafür wird die Intensität $I_{\nu}$ innerhalb der Emisssionsregion in einen ausgehenden $\left(0^{\circ} \leq \vartheta<90^{\circ}\right)$ und in einen einfallenden $\left(90^{\circ} \leq \vartheta \leq 180^{\circ}\right)$ Anteil aufgespalten und die Strahlungstransportgleichung in zwei Differentialgleichungen zweiter Ordnung mit den zugehörigen Randbedingungen umgeformt. Die Lösung dieses Gleichungspaars zu einer vorgegebenen Schichtung von Dichte und Temperatur liefert das Strahlungsfeld der Emissionsregion. Eine Umsetzung des Verfahrens wurde von U. Woelk zur Berechnung der teilchengeheizten Atmosphäre magnetischer weißer Zwerge programmiert (Woelk 1991) und nach Modifikationen auf die Akkretionsregion der Polare angewendet (Woelk \& Beuermann 1996). Dabei wird vorausgesetzt, daß der Strahlungstransport in einer plan-parallelen, unendlich ausgedehnten Schicht stattfindet, und Effekte durch begrenzende seitliche Ränder einer endlichen Region vernachlässigt werden. Eine Version dieses Programms wurde für die vorliegende Arbeit benutzt.

Das Teilprogramm der Hydrodynamik liefert eine Dichte- und Temperaturschichtung der Emissionsregion, die als Grundlage für die Berechnung des Strahlungstransports dient, woraus eine Bestimmung des Strahlungsverlustterms in der Energiegleichung der Elektronen bei einer neuerlichen Lösung der Hydrodynamik folgt. In einem iterativen Verfahren, mit dem Ziel der Energieerhaltung wird die endgültige Struktur der Emissionsregion zu den Parametern $M_{\mathrm{wd}}, B$ und $\dot{m}$ gesucht. Dabei erweist sich die Vorgehensweise über einen weiten Bereich dieser Parameter als stabil.

\subsection{Ergebnisse}

\subsubsection{Schichtung der Emissionsregion}

Mit der oben beschriebenen Methode wurden die Schichtungen der Emissionsregion für den in Polaren zu erwartenden Parameterbereich in Massen $M_{\text {wd }}$, Magnetfeldstärken $B$ und Massenstromdichten $\dot{m}$ berechnet. Die gefundenen Strukturen zeigen, unabhängig von der speziellen Wahl der Parameter, charakteristische Eigenschaften (Abb. 3.3, folgende Seite): Im Bereich nach dem hydrodynamischen Stoß werden die kühlen Elektronen durch die Coulomb-Wechselwirkung mit den heißeren Ionen aufgeheizt, so daß sich die Temperatur der Teilchen annähert. Wegen der Strahlungskühlung der Elektronen verlieren diese jedoch ständig an Energie. Das Wechselspiel dieser Prozesse resultiert in einem ausgeprägten Maximum der Elektronentemperatur bevor der Ausgleich der Teilchentemperaturen eingetreten ist. Nach der thermischen Äquipartition der Teilchen setzt die Coulomb-Wechselwirkung aus, und der Plasmastrom verhält sich wie ein Ein-TeilchenPlasma. Unter weiterer Kühlung fällt die Temperatur rasch ab; der Strom kommt bei einer maximalen Schichtdicke zur Ruhe.

Die Ausprägung der einzelnen Charakteristika hängt nun von dem betrachteten Parametersatz ab. Die Rechnungen ergaben allerdings, daß die qualitativen Aussagen der resultierenden Schichtungen bezüglich einer Erhöhung der Masse des weißen Zwergs mit denen der Vergrößerung der Massenstromdichte übereinstimmen: Ein Anwachsen dieser 


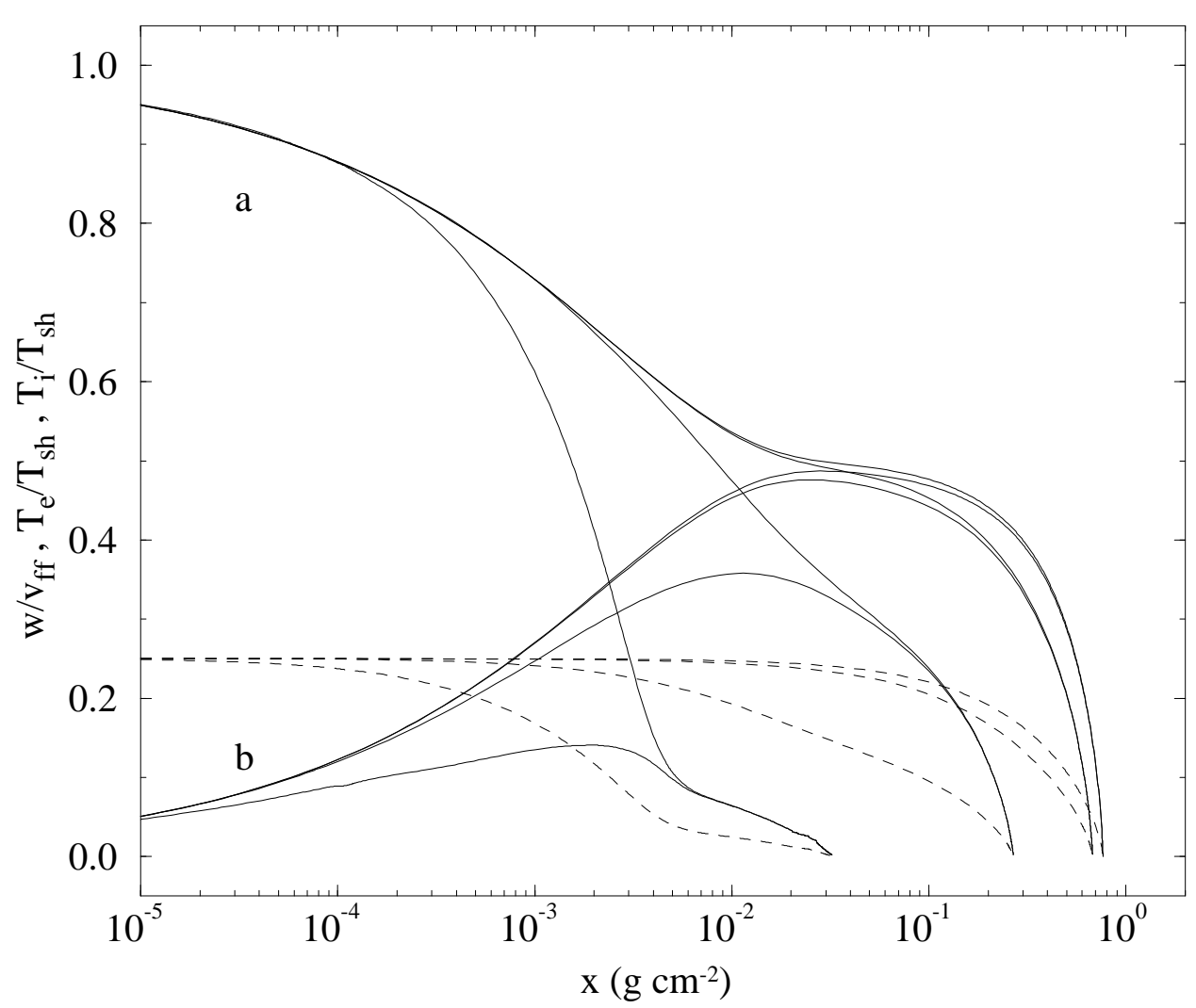

Abbildung 3.3: Temperatur- und Geschwindigkeitsverlauf in der Akkretionsregion für einen weißen Zwerg mit $M_{\mathrm{wd}}=0,6 M_{\odot}$ und $\dot{m}=1 \mathrm{~g} \mathrm{~s}^{-1} \mathrm{~cm}^{-2}$. Dargestellt sind normierte ( $T_{\mathrm{sh}}$ nach Gl. 1.7 mit $\mu=1$ ) Ionen- (a) und Elektronentemperatur (b), sowie die normierte $\left(v_{\mathrm{ff}}\left(R_{\mathrm{wd}}\right)\right)$ Geschwindigkeit (gestrichelte Linien) gegen die Schichtdicke für den Fall reiner Bremsstrahlungskühlung (Kurven größter Ausdehnung in Schichtdicke) und zusätzlicher Zyklotronverluste in einem Magnetfeld mit $B=10,30$ bzw. $100 \mathrm{MG}$ (sukzessive Abnahme der maximalen Schichtdicke).

Parameter erhöht die maximale Elektronentemperatur, da die bei der Akkretion eingebrachte Energie zunimmt $\left(L_{\text {acc }} \sim \dot{M} M_{\text {wd }}^{4 / 3}\right.$, mit der Masse-Radius-Beziehung nichtrelativistisch entarteter weißer Zwerge $R_{\mathrm{wd}} \sim M_{\mathrm{wd}}^{-1 / 3}$ ) und erzeugt eine weiter ausgedehnte Schicht, d.h. eine größere maximale Schichtdicke. Daher soll im folgenden die Struktur der Emissionsregion exemplarisch für die Masse $M_{\mathrm{wd}}=0,6 M_{\odot}$ und Variation von $B$ und $\dot{m}$ dargestellt werden. Die Graphen werden der Übersicht halber auf die Angabe der Temperatur- und Geschwindigkeitsstrukturen beschränkt. Die zugehörige Dichtestruktur ergibt sich in einfacher Weise aus der Kontinuitätsgleichung (Gl. 3.16), bei konstanten $\dot{m}$ somit aus der Invertierung der Geschwindigkeitsstruktur.

Abbildung 3.3 veranschaulicht den Einfluß der Magnetfeldstärke auf die Struktur der Emissionsregion, wenn die Massenstromdichte und die Masse des weißen Zwergs konstant gehalten werden. Die bei der Akkretion eingebrachte Energie ist in diesem Fall konstant; die Strahlungsprozesse bestimmen die Kühlung und damit den Temperaturverlauf der stoßgeheizten Region. 


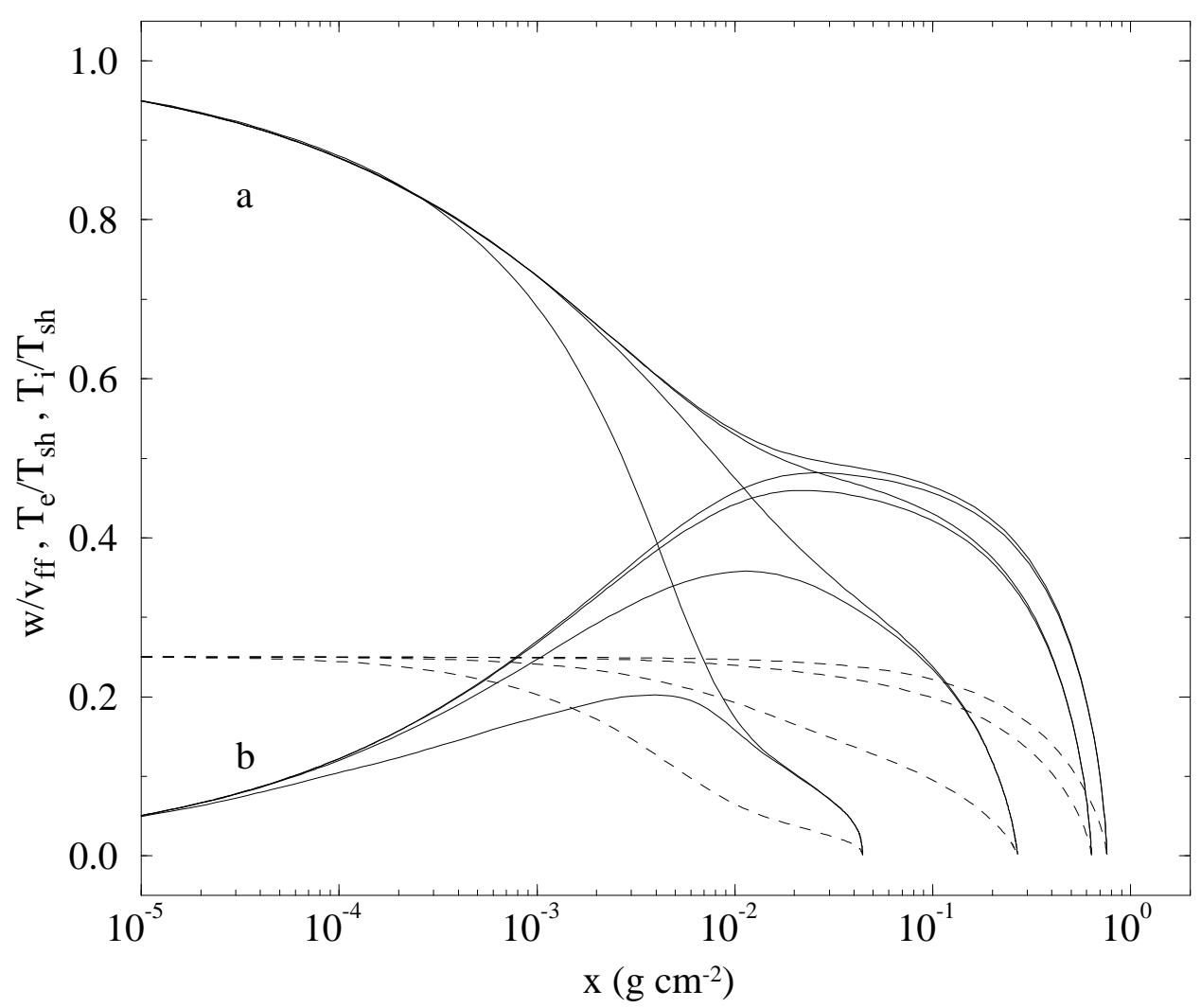

Abbildung 3.4: Darstellung der Temperatur- und Geschwindigkeitsverläufe wie in Abbildung 3.3, aber für ein konstantes Magnetfeld mit $B=30 \mathrm{MG}$ und $\dot{m}=100,10,1$ bzw. $0,1 \mathrm{~g} \mathrm{~s}^{-1} \mathrm{~cm}^{-2}$ (Kurven sukzessiver Abnahme der maximalen Schichtdicke).

Bei vernachlässigter Zyklotronstrahlung wird (unter den betrachteten Annahmen) optisch dünne Bremsstrahlung als einziger Kühlprozeß wirksam. Deren frequenzintegrierte Emissivität ist stärker von der Dichte des Materials, als von der Elektronentemperatur abhängig $\left(j^{\mathrm{ff}} \sim \rho^{2} T^{1 / 2}\right)$ und beeinflußt die Schichtung wesentlich erst im Bereich der Abbremsung des Stroms in der Nähe der Sternoberfläche. Dort sammelt sich das Plasma an, wobei der starke Anstieg der Dichte durch die Kontinuitätsgleichung (Gl. 3.16) beschrieben wird. Dies erhöht die Strahlungskühlung und führt somit zu einer raschen Temperaturabnahme des Plasmas in diesem Bereich.

Wird Zyklotronstrahlung als weiterer Strahlungsprozeß hinzugenommen (in Abb. 3.3 Kurven zu $B=10,30$ bzw. $100 \mathrm{MG}$ ), erhöht sich die Effizienz der Kühlung in der Emissionsregion. Eine qualitative Abschätzung der zugehörigen Strahlungsverluste liefert (siehe Kap. 2.2): $\underline{\partial} \cdot \underline{F}_{\text {cyc }} \sim \rho^{0,15} T^{2,5} B^{2,85}$. Damit ist Zyklotronstrahlung mit hoher Potenz von der Magnetfeldstärke und stärker von der Elektronentemperatur als von der Dichte abhängig. Im Gegensatz zur Bremsstrahlung kann die Kühlung daher (bei hinreichend hoher Magnetfeldstärke) schon in Bereichen leicht erhöhter Temperatur der Elektronen, also am Anfang der geheizten Schicht, einsetzen. Die effizientere Kühlung resultiert in ei- 
ner deutlichen Absenkung der Maximaltemperatur der Elektronen um einen Faktor 5 bei einem 10 mal größeren Magnetfeld. In ähnlich starker Weise wird die maximale Schichtdicke, und damit die Ausdehnung der Emissionsregion, beeinflußt.

Die unterschiedliche Abhängigkeit der betrachteten Strahlungsprozesse von den physikalischen Größen verändert weiterhin die Form der Verläufe der Temperatur und Geschwindigkeit. Bei einer relativ geringen Magnetfeldstärke von $B=10 \mathrm{MG}$ ähnelt die Schichtung den durch reine Bremsstrahlung erzeugten Kurven: Da die Dichte, und damit die Kühlung durch Bremsstrahlung, zum Ende der geheizten Region stark zunimmt, fallen Temperatur und Geschwindigkeit in diesem Bereich rasch ab; Zyklotronstrahlung ist bei kleinen Feldstärken ein im Vergleich zur Bremsstrahlung unbedeutender Verlustprozeß.

Bei einer Erhöhung der Magnetfeldstärke wird die geheizte Schicht in zunehmendem Maß durch Zyklotronstrahlung gekühlt, und Bremsstrahlung ist von geringer werdender Bedeutung. Da die Zyklotronstrahlung in stärkerer Weise von $B$ und von der Temperatur als von der Dichte abhängt, bestimmen diese Größen die Strahlungskühlung. Für eine Zunahme der Magnetfeldstärke liegt die resultierende Temperaturverteilung daher - über die gesamte geheizte Region hinweg - deutlich unter dem Verlauf einer bremsstrahlungsdominierten Schichtung. Die schon kurz nach dem Stoß einsetzende Kühlung sorgt darüberhinaus für ein langsames Ansteigen der Elektronentemperatur, d. h. für eine flachere Kurve bis zum Temperaturmaximum als bei kleinen Magnetfeldern. In der Umgebung dieses Maximums sind die Zyklotronverluste am stärksten, so daß mit zunehmender Feldstärke dieser Bereich stärker lokalisiert ist. Die folgende Temperaturabnahme bis zum vollständigen Abbremsen des Akkretionsstroms verringert auch die Zyklotronstrahlungsverluste, die bei hohen Magnetfeldern allerdings weiterhin effektiver sind als die Bremsstrahlungsverluste. Somit fällt die Temperatur zum Ende der Emissionsregion weniger stark ab als bei der dichteabhängigen Kühlung durch Bremsstrahlung: Mit ansteigender Magnetfeldstärke wird der Verlauf der Temperaturkurve am Ende der geheizten Region zunehmend flacher. Erst kurz bevor der Strom zur Ruhe gekommen ist, erzeugt die drastisch ansteigende Dichte eine starke Bremsstrahlungskühlung und bringt die Temperatur auf einen Endwert. In diesem Bereich der Emissionsregion überwiegen die Bremsstrahlungsverluste die der Zyklotronstrahlung - auch bei hohen Feldstärken.

Somit zeigt sich am Ende der geheizten Schicht der für die gewählten Parameter dominante Strahlungsprozeß: Im Fall der reinen Bremsstrahlung verursacht die stark anwachsende Dichte den raschen Temperaturabfall, während bei einem hohen Magnetfeld die Zyklotronkühlung einen flacheren Temperatur- und Geschwindigkeitsverlauf erzeugt.

In Abbildung 3.4 (vorherige Seite) sind die Temperatur- und Geschwindigkeitsverläufe für eine konstante Magnetfeldstärke $(B=30 \mathrm{MG})$ und Variation der Massenstromdichte $\dot{m}$ dargestellt. Hierbei ist die eingebrachte Akkretionsenergie nicht mehr konstant, sondern erhöht sich mit zunehmender Massenstromdichte und sorgt so für ein entsprechendes Anwachsen der maximalen Elektronentemperatur bzw. der Vergrößerung der Schichtdicke am Ende der geheizten Region.

Für die höchste dargestellte Massenstromdichte $\left(\dot{m}=100 \mathrm{~g} \mathrm{~s}^{-1} \mathrm{~cm}^{-2}\right)$ ergibt sich nach der Kontinuitätsgleichung (Gl. 3.16) eine Dichtestruktur, bei der die Bremsstrahlungsverluste die zu der gewählten Magnetfeldstärke $(B=30 \mathrm{MG})$ auftretenden Verluste durch 
Zyklotronstrahlung überwiegen. Die resultierende Schichtung ähnelt daher dem Ergebnis für reine Bremsstrahlung aus Abbildung 3.3 (Seite 48).

Eine Verringerung der Massenstromdichte (in Abb. 3.4 Kurven zu $\dot{m}=10,1$ bzw. $0,1 \mathrm{~g} \mathrm{~s}^{-1} \mathrm{~cm}^{-2}$ ) führt zu Dichteverläufen bei denen der Anteil der Bremsstrahlung an den Strahlungsverlusten der Emissionsregion veringert wird, und damit Zyklotronstrahlung der dominante Verlustprozeß wird. Ähnlich zu den Kurven ansteigender Feldstärke in Abbildung 3.3 zeigt sich auch hier die Abnahme der maximalen Elektronentemperatur und Verringerung der Ausdehnung der geheizten Schicht. Insbesondere ist bei der kleinsten dargestellten Massenstromdichte der als Ausprägung einer vorherrschenden Zyklotronkühlung beschriebene flache Verlauf der Elektronentemperatur am Anfang und Ende der Region zu sehen. Eine weitere Verringerung von $\dot{m}$ würde bei dem gewählten Wert für $B$ auf eine Kurve ähnlich der zu $B=100 \mathrm{MG}$ in Abbildung 3.3 führen, da die Zyklotronstrahlung weiter an Bedeutung gewinnt.

Die Veränderung von $\dot{m}$ ergibt damit Schichtungen, die in qualitativer Übereinstimmung mit den Ergebnissen aus der Magnetfeldvariation sind und das erwartete Verhalten der Temperaturschichtung in der Akkretionsregion wiedergeben: Bei Erhöhung der Effizienz der Strahlungsverluste wird die Temperatur in der Emissionsregion abgesenkt, und das geheizte Gebiet ist weniger weit ausgedehnt.

\subsubsection{Spektrum der Emissionsregion}

Die bei der Berechnung der Struktur der Akkretionsregion berücksichtigten Strahlungsprozesse erzeugen das Gesamtspektrum der Emissionsregion und damit deren Strahlungsverluste. Je nach Wahl der Parameter $M_{\text {wd }}, \dot{m}$ und $B$ und der sich daraus ergebenden Schichtung der physikalischen Größen, wird das Spektrum - für die oben betrachteten Prozesse - durch Brems- bzw. Zyklotronstrahlung dominiert. Dieses Verhalten soll im folgenden anhand des Spektrums der Emissionsregion zu den Parametern der in den Abbildungen 3.3 und 3.4 dargestellten Strukturen beschrieben werden. Um die Strahlungsverluste, die in die Berechnung dieser Schichtungen eingegangen sind, zu erfassen, wurde die Strahlungstransportgleichung (Gl. 3.23) mit den vorgegebenen Schichtungen unter den gleichen Bedingungen gelöst, die auch in das Gleichungssystem (Gln. 3.20 bis 3.23) eingegangen sind. Insbesondere wurde angenommen, daß die Emissionsregion nicht durch Randeffekte einer seitlichen Begrenzung des geheizten Gebiets beeinflußt wird. Die Strahlung passiert daher unter jedem Sichtwinkel zur Normalen (d. h. zur lokalen Magnetfeldrichtung) die gesamte Höhenausdehnung der Emissionsregion. Dies entspricht dem Idealfall einer unendlich ausgedehnten, plan-parallelen Akkretionsregion endlicher Höhe.

Die im folgenden dargestellten Spektren enthalten nicht die in Polaren weiterhin zu beobachtenden Beiträge des weißen Zwergs, des Sekundärsterns und des Akkretionsstroms. Sie sind daher nicht ohne weiteres mit Beobachtungen eines konkreten AM Her Systems zu vergleichen, sondern stellen nur die aus der Akkretionssäule stammende Strahlung dar.

In Abbildung 3.5 (folgende Seite) sind die resultierenden Spektren zu den Schichtungen aus Abbildung 3.3 aufgetragen. Dargestellt ist der winkelabhängige, spektrale Fluß $F_{\nu}$ gegen die Frequenz $\nu$ für den Fall reiner Bremsstrahlung und zu den Magnetfeldstärken 


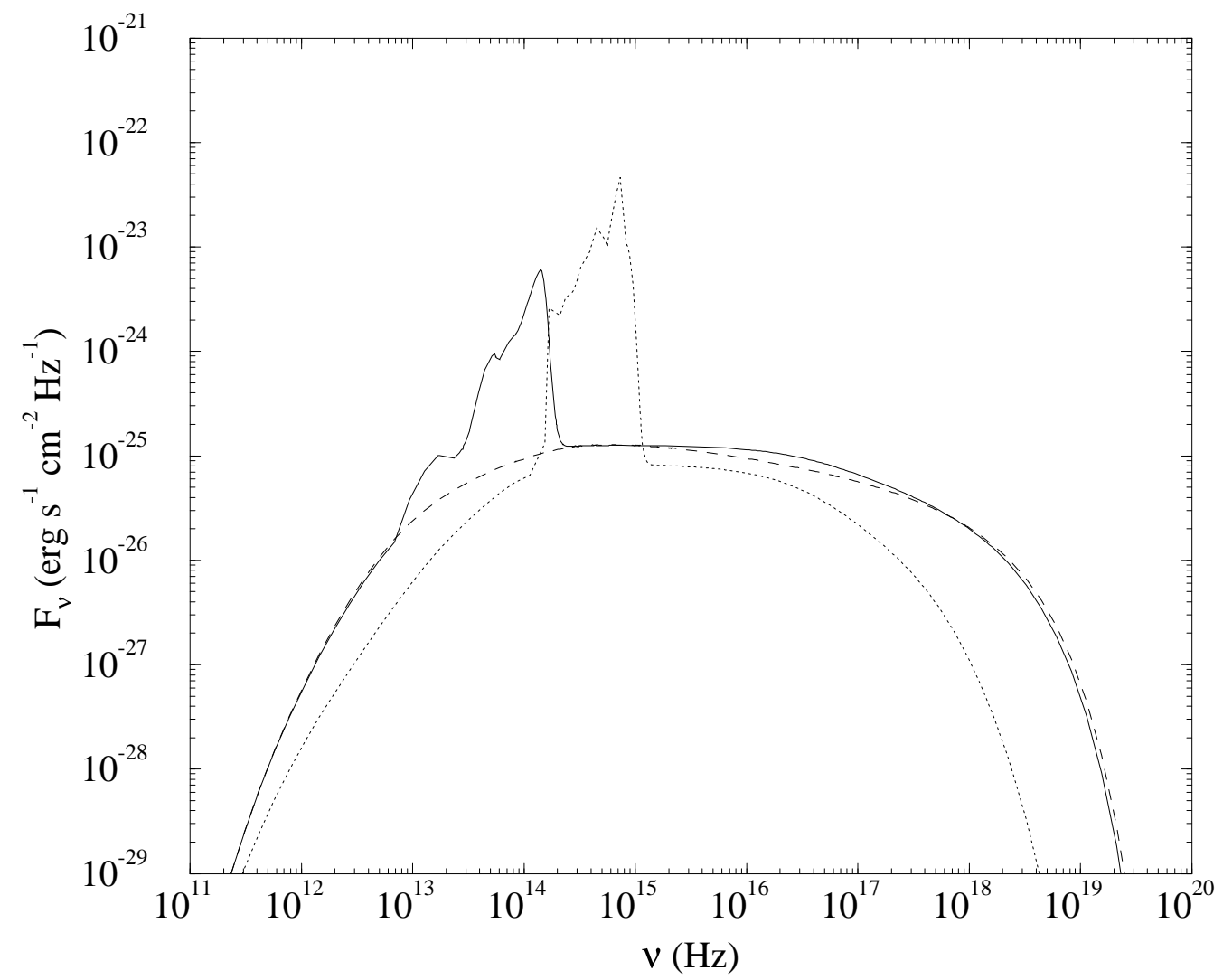

Abbildung 3.5: Spektrum der Emissionsregion zu den Schichtungen aus Abbildung $3.3\left(M_{\mathrm{wd}}=0,6 M_{\odot}, \dot{m}=1 \mathrm{~g} \mathrm{~s}^{-1} \mathrm{~cm}^{-2}\right)$. Dargestellt ist der spektrale Fluß $F_{\nu}$ gegen die Frequenz $\nu$ für den Fall reiner Bremsstrahlungskühlung (gestrichelte Linie) und zusätzlicher Zyklotronverluste in einem Magnetfeld mit $B=10$ bzw. $100 \mathrm{MG}$ (durchgezogene bzw. gepunktete Linie). Der Sichtwinkel zum Magnetfeld beträgt $\vartheta=5^{\circ}$.

$B=10$ bzw. 100 MG. (Der Übersicht halber ist das Spektrum zu $B=30 \mathrm{MG}$ fortgelassen worden; es findet sich jedoch in Abb. 3.6.) Zur Umrechnung der aus der Strahlungstransportgleichung hergeleiteten Intensität in einen Fluß wurde eine Akkretionsfläche von $A_{\text {acc }}=10^{16} \mathrm{~cm}^{2}\left(f \approx 10^{-3}\right)$ auf dem weißen Zwerg angenommen und die Entfernung zu $d=10 \mathrm{pc}$ festgelegt. Der Sichtwinkel zur Flächnenormale der Akkretionsfläche, und damit zum als parallel dazu angenommenen Magnetfeld, beträgt $\vartheta=5^{\circ}$.

Das Spektrum zur Schichtung für reine Bremsstrahlungskühlung wird bei hohen Frequenzen $\left(\nu>10^{15} \mathrm{~Hz}\right)$ durch die optisch dünne Emission dieses Strahlungsprozesses dominiert. Dessen Emissivität ist gegeben durch (Rybicki \& Lightman 1979):

$$
j_{\nu}^{\mathrm{ff}} \sim \frac{\rho^{2}}{T^{1 / 2}} e^{-\frac{h \nu}{k_{\mathrm{B}} T}}
$$

Das resultierende Spektrum entsteht nun durch die Überlagerung dieser Strahlungsbeiträge aus den Teilbereichen der Temperatur- und Dichteschichtung. Die Gebiete um das Temperaturmaximum erzeugen dabei den hochenergetischen Anteil der Strahlung, tragen 


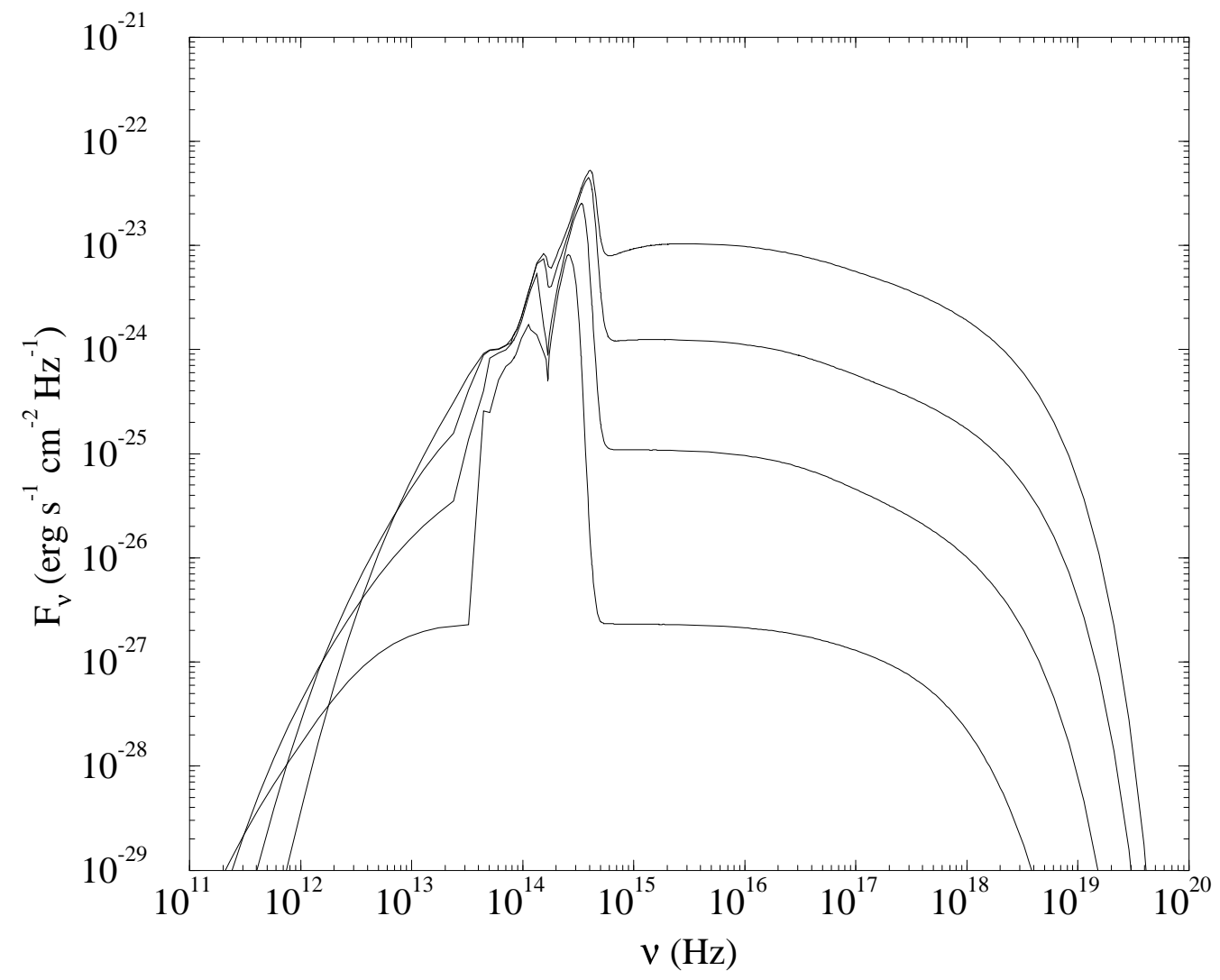

Abbildung 3.6: Darstellung des Spektrums der Emissionsregion zu den Schichtungen aus Abbildung 3.4 für ein konstantes Magnetfeld mit $B=30 \mathrm{MG}$ und $\dot{m}=100,10,1$ bzw. $0,1 \mathrm{~g} \mathrm{~s}^{-1} \mathrm{~cm}^{-2}$. Die obere Kurve gibt den spektralen Fluß $\left(\vartheta=5^{\circ}\right)$ der höchsten, die untere Kurve den der kleinsten Massenstromdichte an.

aber wegen der relativ geringen Dichte nur wenig zum Gesamtfluß bei. In den Regionen hoher Dichte am Ende der Emissionsregion ist die Temperatur dagegen sehr klein, und ihr Beitrag findet sich hauptsächlich bei mittleren Frequenzen (in den Spektren in Abb. 3.5 etwa um $\nu \approx 10^{15} \mathrm{~Hz}$ ). Der stetige Temperatur- und Dichteverlauf zwischen diesen beiden Extrempunkten führt nun zu dem Flußverlauf für hohe Frequenzen.

Es ist gerechtfertigt die Bremsstrahlung bei hohen Frequenzen als optisch dünn anzunehmen, denn für den Absorptionskoeffizienten findet man (Rybicki \& Lightman 1979):

$$
\kappa_{\nu}^{\mathrm{ff}} \sim \frac{\rho^{2}}{\nu^{3} T^{1 / 2}}\left[1-e^{-\frac{h \nu}{k_{\mathrm{B}} T}}\right]
$$

und bei einer vorgegebenen Temperatur- und Dichteverteilung, in einem Medium konstanter Ausdehnung (mit geometrischer Tiefe $S$ ), gilt für die optische Tiefe $\tau_{\nu}(S):=\kappa_{\nu} S \ll 1$ oberhalb einer bestimmten Frequenz. Im optischen und infraroten Spektralbereich ist diese Annahme jedoch nicht mehr gültig, und Bremsstrahlung wird im Medium absorbiert $\left(\tau_{\nu} \gg 1\right)$. Die Photonen werden dabei thermalisiert; das optisch dicke Medium strahlt bei diesen Frequenzen wie ein Schwarzkörper $B_{\nu}(T)$ mit der lokalen Temperatur $T$. Im Fall der geheizten Schicht tritt dieses Szenario am Anfang der Emissionsregion auf, da 
wegen der dort vorkommenden Temperaturen, bei fast konstanter Dichte, der Bremsstrahlungsabsorptionskoeffizient für kleine Frequenzen groß wird. Ist bei einer Frequenz $\nu_{1}$ die Bedingung $\tau_{\nu_{1}}\left(S_{1}\right) \gg 1$ in der geometrischen Tiefe $S_{1}$ erfüllt, wird das Spektrum durch $B_{\nu_{1}}\left[T\left(S_{1}\right)\right]$ gebildet. Da der Absorptionskoeffizient umgekehrt proportional zur Frequenz ist, wird für eine Frequenz $\nu_{2}>\nu_{1}$ die optische Tiefe $\tau_{\nu_{2}}\left(S_{2}\right) \gg 1$ aber erst tiefer in der Schicht erreicht: $S_{2}>S_{1}$. Wegen des Temperaturanstiegs zum Maximum hin gilt aber auch: $B_{\nu_{2}}\left[T\left(S_{2}\right)\right]>B_{\nu_{1}}\left[T\left(S_{1}\right)\right]$. Das Spektrum wird also in diesem Spektralbereich durch eine Überlagerung der Schwarzkörperstrahlung - gemäß der Temperaturschichtung - gebildet und erklärt damit den Flußverlauf der Bremsstrahlung in Abbildung 3.5 für Frequenzen $\nu<10^{13} \mathrm{~Hz}$.

Wird bei der Kühlung der Akkretionsregion Zyklotronstrahlung als zusätzlich zur Bremsstrahlung autretender Verlustprozeß zugelassen, so tritt diese als weitere Komponente im Gesamtspektrum auf. Als deren kennzeichnende Eigenschaften kann man die von der Magnetfeldstärke abhängige spektrale Lage und harmonische Struktur der Emissivität ansehen (siehe z. B. Bekefi 1966). Der spektrale Ort der 1. Harmonischen wird durch die Zyklotronfrequenz $\nu_{\mathrm{c}}$ festgelegt:

$$
\nu_{\mathrm{c}}=\frac{e B}{2 \pi m_{\mathrm{e}} c}=2,8 \cdot 10^{12} \mathrm{~Hz}\left(\frac{B}{1 \mathrm{MG}}\right) ;
$$

die folgenden Harmonischen liegen bei Vielfachen dieser Frequenz. Dementsprechend zeigen die Spektren zu den Schichtungen für die Magnetfeldstärken $B=10$ bzw. $100 \mathrm{MG}$ in Abbildung 3.5 eine Verschiebung, des durch die Zyklotronstrahlung verursachten Flußanstiegs: Während für $B=10 \mathrm{MG}$ die Abweichungen von der Bremsstrahlungskurve bei $\nu \approx 10^{13} \mathrm{~Hz}$ einsetzen, liegt dieser Punkt für das 10mal größere Magnetfeld bei $\nu \approx 10^{14} \mathrm{~Hz}$.

Wegen der harmonischen Struktur der Emissivität, deren Maxima von einer zu hohen Harmonischen abfallenden Einhüllenden begrenzt werden, zeigt auch der Zyklotronstrahlungsabsorptionskoeffizient (im LTE) diesen Verlauf. Er wird weiterhin dadurch beeinflußt, daß Absolutwert und Breite einer individuellen Harmonischen mit zunehmender Temperatur größer werden, wenn der Sichtwinkel zum Magnetfeld konstant bleibt $\left(\vartheta=5^{\circ}\right.$ in Abb. 3.5). Bei einer vorgegebenen Temperaturstruktur nimmt der Absorptionskoeffizient daher bis zum Maximum der 1. Harmonischen zu, was einer Zunahme der optischen Tiefe entspricht. Bis zu der Frequenz, bei der $\tau_{\nu}>1$ gilt, wird das Spektrum durch die aufintegrierten Beiträge der Emissivität zu den verschiedenen Temperaturen gebildet. Oberhalb dieser Frequenz nimmt die optische Tiefe dagegen rasch zu, und das optisch dicke Spektrum entsteht durch Schwarzkörperstrahlung der lokalen Temperatur. Eine analoge Argumentation wie im oben dargestellten Fall der Bremsstrahlung unter Berücksichtigung der Vergrößerung des Absorptionskoeffizienten mit der Frequenz zeigt nun, daß die Strahlung in der Nähe des Harmonischenmaximums aus den vorderen Bereichen der Temperaturstruktur stammt, der Schwarzkörperstrahlungsfluß also mit der Temperatur abnimmt. Dies verursacht die in Abbildung 3.5 bis zum Zentrum der 1. Harmonischen $\left(\nu \approx 2 \cdot 10^{13} \mathrm{~Hz}\right.$ für $\left.B=10 \mathrm{MG}\right)$ festzustellende Flußabnahme. Nach Erreichen des Maximalwertes des Absorptionskoeffizienten bei der Zyklotronfrequenz $\nu_{\mathrm{c}}$ nimmt die opti- 
sche Tiefe wieder ab, die optisch dicke Strahlung entstammt Bereichen größer werdender Temperatur, der Fluß steigt erneut an, bis die gesamte Schicht optisch dünn wird und die temperaturabhängige Emissivität das Spektrum bildet. Dieser Vorgang wiederholt sich bei jeder Harmonischen, wobei der jeweilige Maximalwert des Zyklotronstrahlungsabsorptionskoeffizienten mit steigender Frequenz abnimmt. Die Strahlung im Kern der hohen Harmonischen entstammt daher immer tiefer liegenden Schichten höherer Temperatur, was sich in der Flußzunahme des Spektrums ausdrückt. Weiterhin sorgt die Abnahme des Maximums des Absorptionskoeffizienten dafür, daß ab einer bestimmten Harmonischen die gesamte geheizte Schicht für höhere Frequenzen optisch dünn ist, und das Spektrum duch Aufintegration der Zyklotronemissivität erzeugt wird.

Die Form des Zyklotronspektrums hängt damit in starker Weise von der Temperaturstruktur ab, die zusätzlich noch dafür sorgt, daß bei insgesamt höheren Temperaturen in der Schicht die einzelnen Harmonischen stärker verbreitert sind als bei niedrigen Temperaturen. In Abbildung 3.5 ist der von der Zyklotronstrahlung überdeckte Spektralbereich daher für die Kurve zu $B=10 \mathrm{MG}$ breiter als bei der aus der Schichtung mit niedrigen Temperaturen stammenden Kurve zu $B=100$ MG. Somit ist die deutliche Sichtbarkeit individueller Harmonischer auch ein Hinweis auf eine Schichtung mit niedriger Maximaltemperatur.

Die Verluste der geheizten Akkretionsregion entstehen durch Überlagerung der Spektren der Brems- und Zyklotronstrahlung. Wie oben beschrieben, ist die Bremsstrahlung ein effektiver Kühlprozeß bei hohen Dichten, während die Zyklotronstrahlung stärker von der Temperatur und dem Magnetfeld abhängt. Bei geringer Feldstärke sind die Unterschiede zwischen dem Gesamtspektrum aus reiner Bremsstrahlungskühlung und dem mit zusätzlicher Zyklotronkühlung (Abb. 3.5) - bezüglich des Energieverlusts der Emissionsregion - auch unwesentlich. Zwar tritt Zyklotronstrahlung als neue Komponente auf, jedoch trägt sie zum Energieinhalt des Gesamtflusses kaum bei. Denn den Hauptbeitrag liefert die harte Bremsstrahlung, und in diesem Bereich sind die Spektren sehr ähnlich. Daher weicht die sich aus dieser Kühlung ergebende Temperaturstruktur nur wenig von der aus reiner Bremsstrahlung resultierenden ab (Abb. 3.3).

Bei zunehmender Magnetfeldstärke geschieht die Kühlung hauptsächlich durch Zyklotronstrahlung, und der Anteil der Bremsstrahlung am Gesamtenergiefluß geht zurück. Das Spektrum zu der hohen Feldstärke von $B=100 \mathrm{MG}$ in Abbildung 3.5 ist daher auch durch den Zyklotronanteil dominiert; die Komponente aus der Bremsstrahlung ist im Vergleich zu dem Spektrum aus reiner Bremsstrahlungskühlung stark reduziert. Der wesentliche Energieinhalt des Gesamtstrahlungsflusses wird nun durch die Zyklotronstrahlung gestellt, der Beitrag aus Bremsstrahlung ist demgegenüber geringer. Wegen der schwachen Dichteabhängigkeit der Zyklotronstrahlung kann die Energie effektiver aus der geheizten Schicht transportiert werden als durch Bremsstrahlung. Die Temperaturstruktur zu der Feldstärke von $B=100 \mathrm{MG}$ in Abbildung 3.3 zeigt daher eine deutlich geringere Maximaltemperatur der Elektronen und geringere Ausdehnung der Emissionsregion als für ein schwaches bzw. vernachlässigbares Magnetfeld.

Die Auswirkung des Magnetfeldes auf die Strahlungskühlung der Emissionsregion wird anhand der über alle Raumwinkel und Frequenzen integrierten Strahlung („Leuchtkraft“) 
beschrieben. In folgender Tabelle sind die Leuchtkraftanteile der Zyklotron- $L_{\text {cyc }}$ und der Bremsstrahlung $L_{\mathrm{ff}}$ für den Fall der reinen Bremsstrahlung $(B=0 \mathrm{MG})$ und zu den Magnetfeldstärken $B=10$ bzw. $100 \mathrm{MG}$ angegeben:

\begin{tabular}{c|c|c}
$B(\mathrm{MG})$ & $L_{\mathrm{cyc}}\left(\mathrm{erg} \mathrm{s}^{-1}\right)$ & $L_{\mathrm{ff}}\left(\mathrm{erg} \mathrm{s}^{-1}\right)$ \\
\hline 0 & 0 & $9,2 \cdot 10^{32}$ \\
10 & $8,7 \cdot 10^{31}$ & $8,3 \cdot 10^{32}$ \\
100 & $8,3 \cdot 10^{32}$ & $9,4 \cdot 10^{31}$
\end{tabular}

Die Zunahme des Zyklotronanteils mit steigendem Magnetfeld ist deutlich sichtbar.

Abbildung 3.6 (S. 53) zeigt den Einfluß der Massenstromdichte $\dot{m}$ auf das Verhältnis der beiden Verlustprozesse bei konstanter Magnetfeldstärke $B=30$ MG. Bei dem Spektrum zu dem höchsten dargestellten Wert $\dot{m}=100 \mathrm{~g} \mathrm{~s}^{-1} \mathrm{~cm}^{-2}$ läßt die resultierende Dichtestruktur die Bremsstrahlung als Kühlprozeß dominieren; Zyklotronstrahlung liefert bei dem relativ geringen Magnetfeld einen verschwindend kleinen Beitrag zum Energiefluß. Die zugehörige Temperaturstruktur in Abbildung 3.4 zeigt demgemäß einen Verlauf, der mit der Schichtung aus Abbildung 3.3 zu reiner Bremsstrahlungskühlung fast identisch ist. Bei Verringerung der Massenstromdichte wird auch der resultierende Dichteverlauf herabgesetzt, so daß der Einfluß der Bremsstrahlung geringer wird. Bei den kleiner werdenden $\dot{m}$ dominiert mehr und mehr die temperaturabhängige Zyklotronstrahlung, die Spektren zeigen deren stärker werdende Komponente. Auch die entsprechenden Strukturen in Abbildung 3.4 werden damit durch den Einfluß der Zyklotronstrahlung geprägt: Die Maximaltemperatur sinkt, und die Ausdehnung der geheizten Schicht verringert sich.

Damit ist der Parameterbereich der einzelnen hier betrachteten Strahlungsverlustprozesse grob umrissen: Für große Magnetfelder $B$ und/oder kleine Massenstromdichten $\dot{m}$ ist Zyklotronstrahlung der dominante Kühlungsprozeß, während Bremsstrahlung den Energieverlust stellt, wenn hohe $\dot{m}$ auftreten und Magnetfelder vernachlässigt werden können. Die Masse des weißen Zwergs $M_{\text {wd }}$ hat dabei einen ähnlichen Einfluß wie $\dot{m}$, da sie wie diese die akkretierte Energie festlegt.

Bisher wurde der Sichtwinkel zum Magnetfeld konstant gehalten, um die grundsätzlichen Auswirkungen der Parameter auf die Strahlungsprozesse und die resultierenden Schichten darzustellen. Da die Zyklotronstrahlung aber ihre Eigenschaften bei Variation des Winkels ändert, soll am Beispiel des Spektrums zu den Parametern $M_{\mathrm{wd}}=$ $0,6 M_{\odot}, \dot{m}=1 \mathrm{~g} \mathrm{~s}^{-1} \mathrm{~cm}^{-2}$ und $B=30 \mathrm{MG}$ kurz auf die Auswirkungen dieses zusätzlichen Parameters eingegangen werden. In Abbildung 3.7 ist der Zyklotronanteil am Gesamtspektrum für drei Sichtwinkel aufgetragen; der Bremsstrahlungsanteil für hohe Frequenzen wurde vernachlässigt, da die Emissionsregion dort optisch dünn ist und Bremsstrahlung isotrop ausstrahlt. (Das komplette Spektrum zu $\vartheta=5^{\circ}$ findet sich in Abbildung 3.6.)

In der Theorie zur Herleitung der Zyklotronemissivität kann gezeigt werden, daß bei einer Vergrößerung des Sichtwinkels die einzelnen Harmonischen schmaler werden und die höheren Harmonischen in ihrer Emissivität ansteigen (sog. „Zyklotron-beaming“). Die Reduzierung der Breite der Harmonischen ist gut in deren Absorptionsstruktur zu sehen. Das Spektrum zu $\vartheta=5^{\circ}$ zeigt um den Kern der 1. Harmonischen eine breite, flache Flußabnahme, während bei einem Winkel $\vartheta=85^{\circ}$ eine scharfe, fast linienartige Absorption 


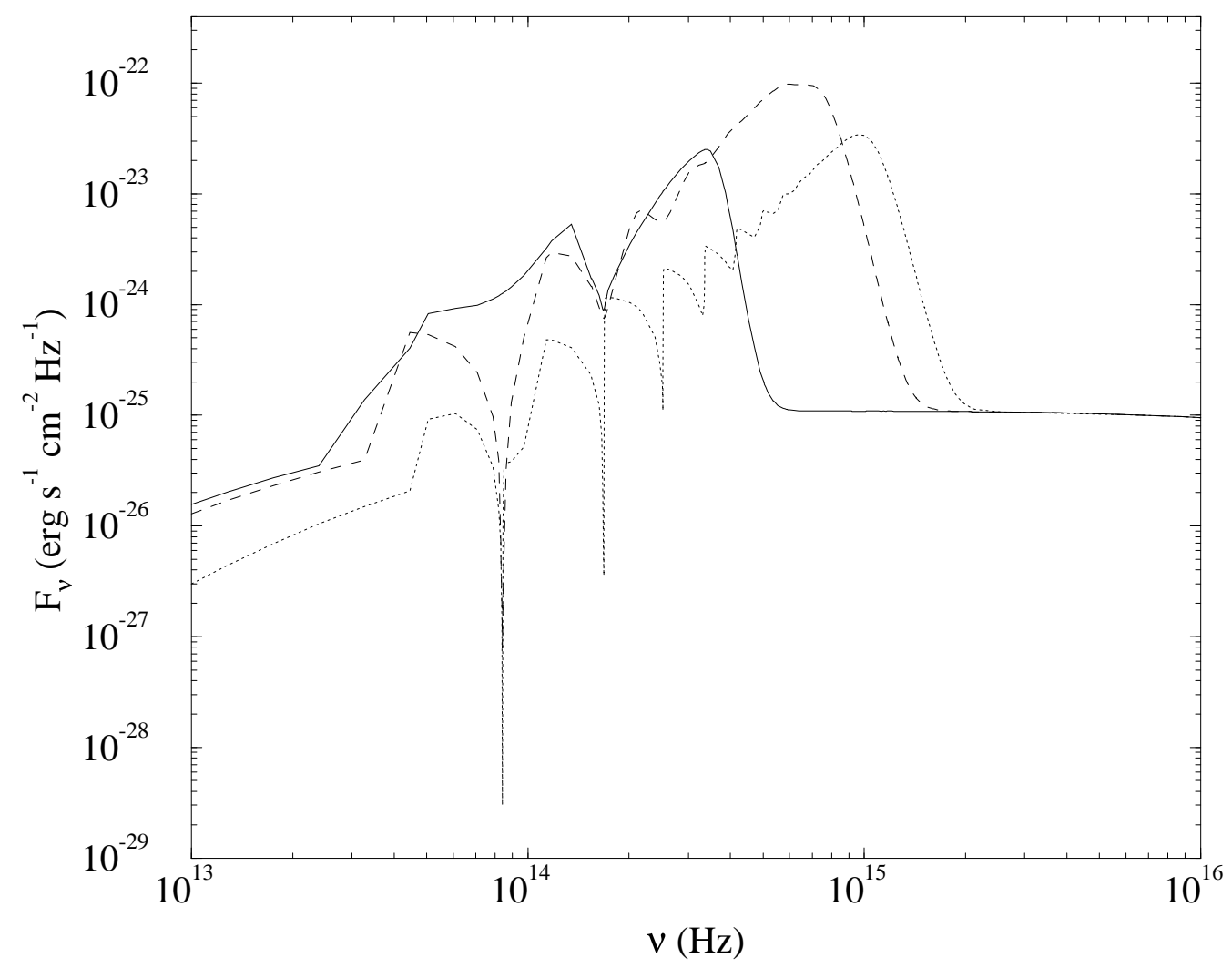

Abbildung 3.7: Spektrum der Emissionsregion für die Schichtung zu den Parametern $M_{\mathrm{wd}}=0,6 M_{\odot}, \dot{m}=1 \mathrm{~g} \mathrm{~s}^{-1} \mathrm{~cm}^{-2}$ und $B=30 \mathrm{MG}$. Aufgetragen ist der spektrale Fluß gegen die Frequenz, für verschiedene Sichtwinkel zum Magnetfeld: $\vartheta=5^{\circ}$ (durchgezogene Linie), $\vartheta=45^{\circ}$ (gestrichelte Linie) und $\vartheta=85^{\circ}$ (gepunktete Linie).

zu beobachten ist. (Die allgemeine Flußabnahme im Absorptionsbereich resultiert aus der Verkürzung der den Fluß erzeugenden Akkretionsfläche auf der Oberfläche des weißen Zwergs durch einen $\cos \vartheta$-Faktor.) Weiterhin sind bei dem großen Sichtwinkel insgesamt mehr absorbierte (niedrige) Harmonische zu sehen, die wegen der Verbreiterung bei kleinen Winkeln zu einem Kontinuum verschwommen waren. Dagegen wird die Zunahme der Emissivität in den hohen Harmonischen an der Verschiebung des Übergangspunktes von Zyklotronstrahlung zum Bremsstrahlungsanteil deutlich. Bei kleinen Sichtwinkeln erzeugen die wegen der Temperaturstruktur zu einem Kontinuum verschmierten hohen Harmonischen Strahlung, die etwa bei $\nu \approx 5 \cdot 10^{14} \mathrm{~Hz}$ schwächer als die Bremsstrahlung wird; mit größer werdendem Winkel verschiebt sich dieser Punkt zu $\nu \approx 2 \cdot 10^{15} \mathrm{~Hz}$. 


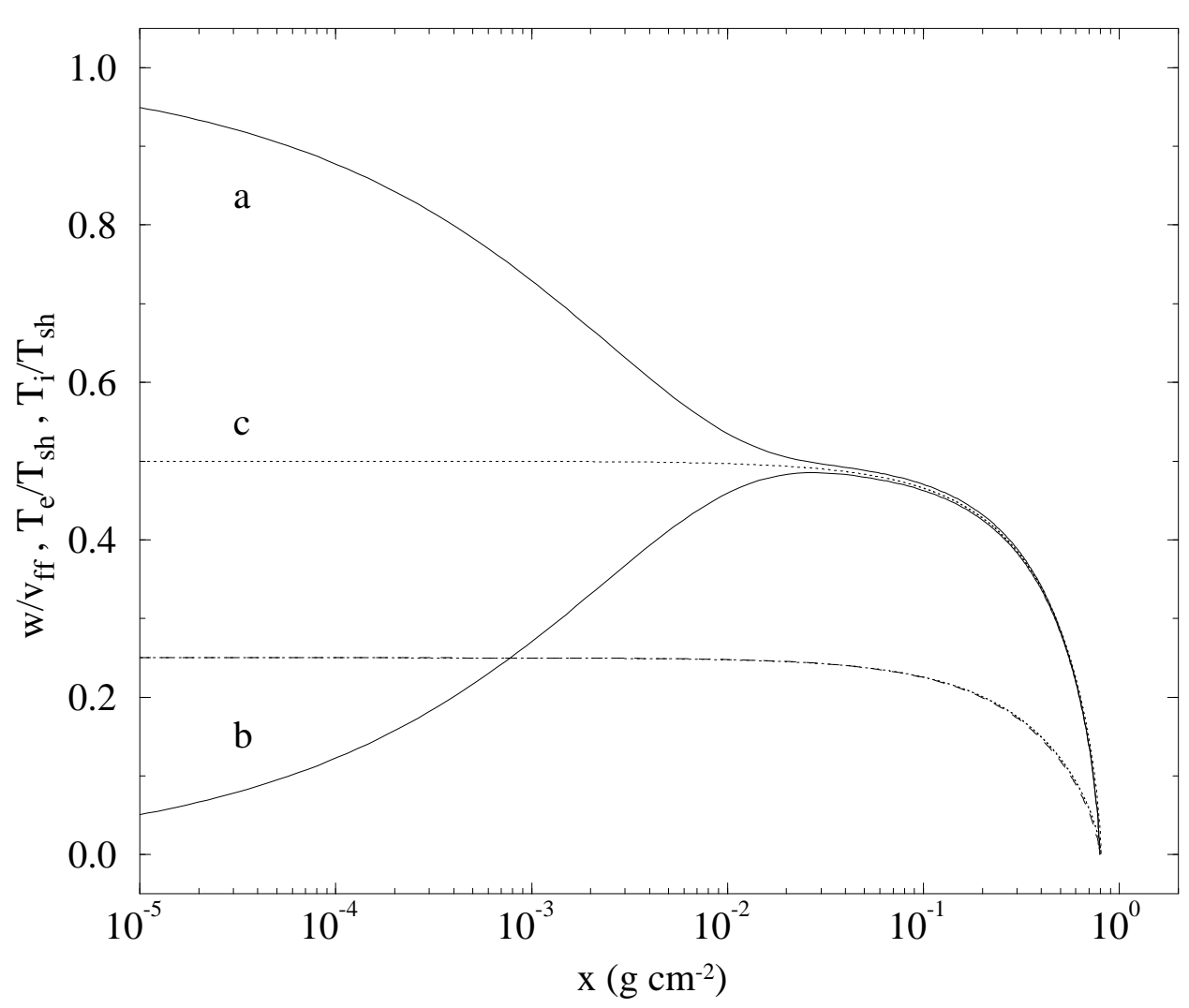

Abbildung 3.8: Vergleich des Temperatur- und Geschwindigkeitsverlaufs eines Ein-Teilchen-Plasmas mit denen eines Zwei-Teilchen-Plasmas bei reiner Bremsstrahlungskühlung zu den Parametern $M_{\mathrm{wd}}=0,6 M_{\odot}$ und $\dot{m}=10 \mathrm{~g} \mathrm{~s}^{-1} \mathrm{~cm}^{-2}$. (a) normierte Ionen-, (b) Elektronentemperatur $\left(T_{\mathrm{sh}}:=3 m_{\mathrm{p}} v_{\mathrm{ff}}^{2} / 16 k_{\mathrm{B}}\right)$ und normierte Geschwindigkeit (gestrichelte Linie) der Zwei-Teilchen-Rechnung. Die Ergebnisse der Ein-Teilchen-Rechnungen sind als gepunktete Linien dargestellt: (c) normierte Elektronentemperatur und Geschwindigkeit (nahezu identisch mit dem Zwei-TeilchenErgebnis).

\subsection{Vergleich mit Ein-Teilchen-Modellen}

\subsubsection{Ein-Teilchen-Plasma mit Bremsstrahlungsverlusten}

Das in den obigen Kapiteln dargestellte Modell eines Zwei-Teilchen-Plasmas, mit einer Unterscheidung von Elektronen- und Ionentemperatur ist notwendig, um die in einer magnetischen Akkretionsregion auftretende Zyklotronstrahlungskühlung korrekt zu erfassen. Besitzt das Magnetfeld jedoch eine vernachlässigbar kleine Feldstärke, oder ist die Massenstromdichte hoch $\left(\dot{m}>10 \mathrm{~g} \mathrm{~s}^{-1} \mathrm{~cm}^{-2}\right.$ für $\left.M_{\mathrm{wd}}=0,6 M_{\odot}\right)$, bestimmt Bremsstrahlung die Kühlung der Emissionsregion. In diesem Fall kann gezeigt werden, daß die Äquipartitionszeitskala $t_{\mathrm{ei}}$ (Gl. 1.13) kleiner ist als die Kühlzeitskala $t_{\mathrm{ff}}$ (Gl. 1.10) und daß ein Ein-Teilchen-Modell gerechtfertigt ist (siehe Kap. 2.1). Dessen Ergebnisse müssen aber als Grenzwert für $B \rightarrow 0$ - auch durch das Zwei-Teilchen-Modell geliefert werden. 


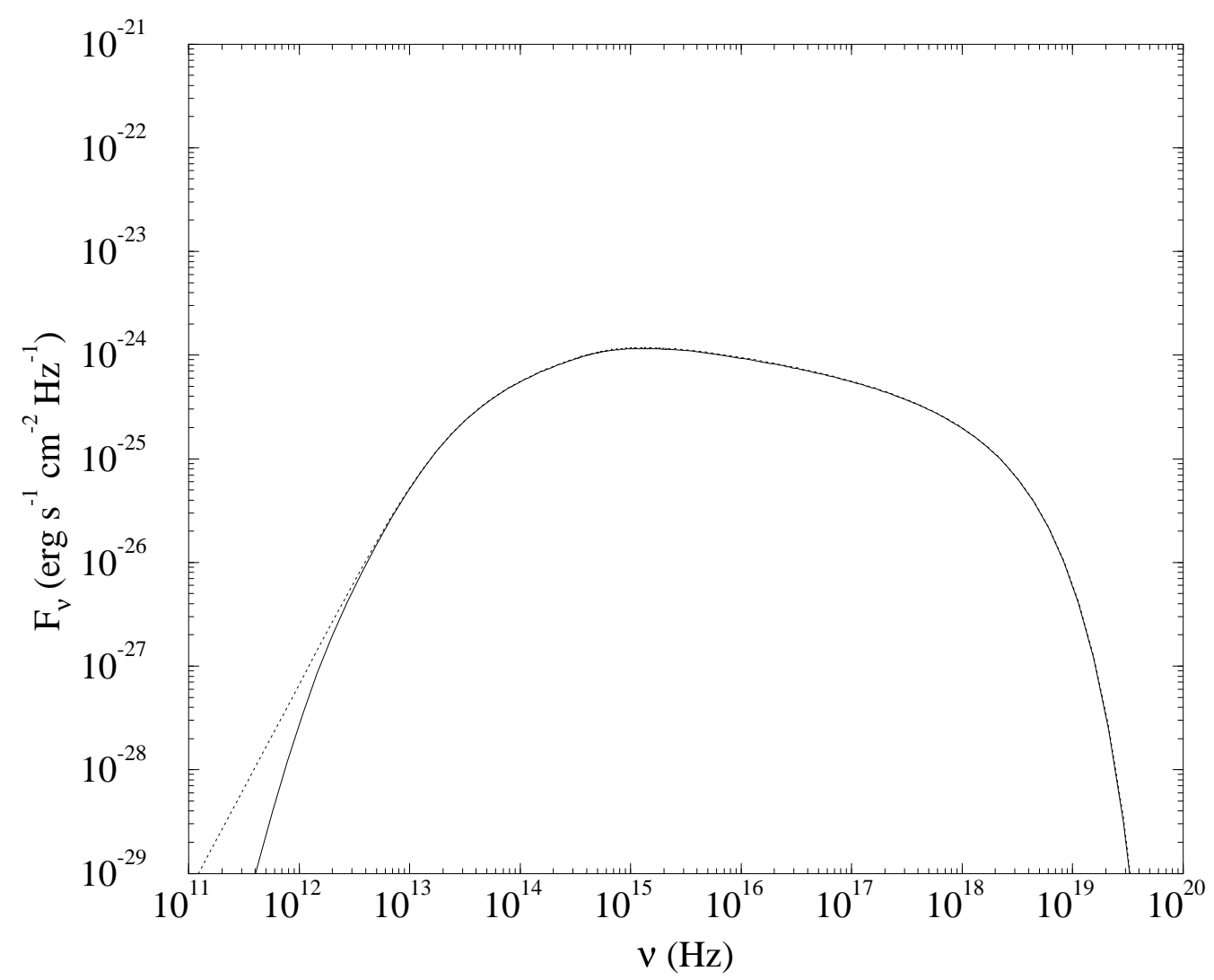

Abbildung 3.9: Bremstrahlungsspektrum zu den Schichtungen aus Abbildung 3.8. Dargestellt ist der spektrale Fluß $F_{\nu}\left(\vartheta=5^{\circ}\right)$ gegen die Frequenz $\nu$ der ZweiTeilchen- (durchgezogende Linie) und der Ein-Teilchen-Rechnung (gepunktete Linie).

Im folgenden soll die Schichtung und das sich daraus ergebende Spektrum der Emissionsregion aus der Beschreibung des Akkretionsstroms als Zwei-Teilchen-Plasma mit der eines Ein-Teilchen-Modells verglichen werden. Dazu wurden die Parameter $M_{\mathrm{wd}}=0,6 M_{\odot}$ und $\dot{m}=10 \mathrm{~g} \mathrm{~s}^{-1} \mathrm{~cm}^{-2}$ gewählt, für die $t_{\mathrm{ei}}<t_{\mathrm{ff}}$ gilt und somit der Ein-Teilchen-Zugang gerechtfertigt ist. Das Zwei-Teilchen-Modell wird in der oben beschriebenen Weise der Iteration zwischen den hydrodynamischen Gleichungen und dem Strahlungstransport berechnet, wobei nun Bremsstrahlung als einziger Kühlprozeß auftritt. Für das Ein-TeilchenModell wird die analytische Lösung der physikalischen Größen der Emissionsregion nach Wu, Chanmugam \& Shaviv (1994) benutzt, deren Parametrisierung mit $\eta_{\text {sh }}=0$ der von Aizu (1973) angegebenen Lösung entspricht (siehe Kap. 2.1 und 2.2).

In Abbildung 3.8 sind die aus den Modellen resultierenden normierten Temperaturund Geschwindigkeitsstrukturen aufgetragen. Die Temperatur wurde auf die in der ZweiTeilchen-Beschreibung definierte Stoßtemperatur $T_{\text {sh }}($ Gl. 1.7 mit $\mu=1$ ) normiert, so daß im Ein-Teilchen-Modell (für vollständig ionisierten Wasserstoff mit $\mu=1 / 2$ ) $T_{\mathrm{sh}} / 2 \mathrm{der}$ Temperaturwert nach dem Stoß ist. Bei dem Zwei-Teilchen-Modell sorgt die CoulombWechselwirkung für den Temperaturangleich von Ionen und Elektronen, bis diese einen 
Maximalwert erreichen, von dem sie durch die Bremsstrahlung herunterkühlen. In der Ein-Teilchen-Beschreibung fehlt diese Wechselwirkung, und der Strom wird vom Temperaturmaximum am Anfang der Schicht durch Strahlung gekühlt. Daraus ergibt sich der unterschiedliche Temperaturverlauf der Ergebnisse kurz nach dem Stoß.

Die Bremsstrahlungskühlung setzt - für die gewählten Parameter - bei einer Schichtdicke von $x \approx 3 \cdot 10^{-2} \mathrm{~g} \mathrm{~cm}^{-2}$ merklich ein, was im Ein-Teilchen-Modell zu einer Temperaturabnahme vom Wert $T_{\mathrm{sh}} / 2$ führt und bei der Zwei-Teilchen-Beschreibung das Maximum der Elektronentemperatur verursacht. Von dieser Schichtdicke an wird auch die Temperaturdifferenz zwischen Ionen und Elektronen ständig geringer. Die wesentliche Kühlung geschieht daher nach der Äquipartition, und eine Unterscheidung zwischen Ionen- und Elektronentemperatur ist nicht mehr sinnvoll: Ein-Teilchen- und Zwei-Teilchen-Beschreibung liefern nahezu identische Temperatur- und Geschwindigkeitskurven im Kühlungsbereich der Emissionsregion. Die dort auftretenden sehr geringen Abweichungen können dadurch erklärt werden, daß die maximale Elektronentemperatur in der Zwei-TeilchenBeschreibung wegen der bei der Coulomb-Wechselwirkung mit den Ionen schon schwach auftretenden Strahlungsverluste leicht unterhalb des Wertes $T_{\mathrm{sh}} / 2$ bleibt. Das Plasma kann von dieser geringeren Temperatur schneller heruntergekühlt werden, und die Schicht ist insgesamt weniger ausgedehnt als in der Ein-Teilchen-Beschreibung.

Die Bremsstrahlungsspektren der Emissionsregion zu den Schichtungen aus den beiden Modellen sind in Abbildung 3.9 (vorherige Seite) dargestellt. Für die Berechnung der Flüsse wurde das in Kapitel 3.4.2 angegebene Verfahren benutzt. Deren spektraler Verlauf ergibt sich in der dort beschriebenen Weise aus der Überlagerung von optisch dicken und dünnen Teilen der unterschiedlich dichten und heißen Gebiete der geheizten Region. Die Übereinstimmung der Spektren ist im optisch dünnen Teil gut, denn auch die Schichtungen stimmen in der sie bildenden Region der hohen Dichten bzw. großen Temperaturen gut überein. Allerdings liefert das Ein-Teilchen-Model einen (in der Darstellung kaum erkennbaren) höheren Fluß, der aus der geringfügig weiter ausgedehnten Schicht und leicht größeren Maximaltemperatur resultiert. Des weiteren zeigen sich Abweichungen bei kleinen Frequenzen $\left(\nu<10^{13} \mathrm{~Hz}\right)$, deren Fluß durch optisch dicke Bremsstrahlung gebildet wird. Das Spektrum der Zwei-Teilchen-Beschreibung entspricht hier dem oben beschriebenen Anstieg des Schwarzkörperflusses mit der ansteigenden Elektronentemperatur. Die Temperaturstruktur im Ein-Teilchen-Modell beginnt dagegen mit der Stoßtemperatur und zeigt keinen Anstieg; für kleine (optisch dicke) Frequenzen entsteht der Fluß daher durch isotherme Schwarzkörperstrahlung zu $T_{\mathrm{sh}} / 2$. Der Übergang zum Flußmaximum wird in beiden Modellen durch Strahlung aus Regionen erzeugt, bei denen die optische Tiefe nicht groß genug ist, um als Schwarzkörper zu strahlen, die aber auch nicht als optisch dünn angesehen werden können.

Die beschriebenen Ergebnisse zeigen, daß zwischen dem Ein-Teilchen- und dem ZweiTeilchen-Modell keine nennenswerte Unterschiede bestehen, vorausgesetzt daß Bremsstrahlung der dominante Kühlungsprozeß der Akkretionsregion ist. Dabei wurde auch deutlich, daß eine Zwei-Teilchen-Beschreibung für Parameter, bei denen eine Ein-TeilchenBeschreibung gerechtfertigt ist, zuverlässige Resultate ergibt, somit also einen Zugang lie- 
fert, um die Akkretionsregion allgemeiner darzustellen, als dies ein Ein-Teilchen-Modell leisten kann.

\subsubsection{Ein-Teilchen-Plasma mit Brems- und Zyklotronstrah- lungsverlusten}

Bei dominanter Bremsstrahlungskühlung läßt sich die Struktur der Akkretionsregion mit einer analytischen Lösung des Differentialgleichungssystems aus hydrodynamischen Gleichungen und aus Energieverlust durch Strahlung angeben. Ist dagegen Zyklotronstrahlung ein nicht zu vernachlässigender Kühlungsprozeß, muß der Energieverlust durch die Lösung der Strahlungstransportgleichung erfaßt werden. Das resultierende Gleichungssystem besteht nun aus Differential- und Integralgleichungen; die Struktur der Akkretionsregion kann somit nur durch aufwendige numerische Verfahren gefunden werden. Eine einfach handhabbare analytische Näherungslösung für die physikalischen Größen in der Emissionsregion ist allerdings möglich und wurde in Kapitel 2.2 beschrieben. Dabei wird davon ausgegangen, daß die Verluste durch Zyklotronstrahlung als deren optisch dicker Anteil darstellbar sind und das Plasma als Ein-Teilchen-Fluid anzusehen ist. Die erste Annahme ist in soweit problematisch, daß eine kritische Frequenz angegeben wird, bis zu der sich die entsprechende Schwarzkörperstrahlung erstreckt und deren Parametrisierung, in den Größen der Emissionsregion, mit Fehlern behaftet ist. Von größerer Einschränkung ist jedoch die Annahme der Ein-Teilchen-Beschreibung, denn eine einfache Abschätzung des Verhältnisses von Äquipartitionszeitskala $t_{\mathrm{ei}}$ zu Kühlzeitskala der Zyklotronstrahlung $t_{\mathrm{cyc}}$ (siehe Gl. 1.14) verdeutlicht, daß schon bei geringen Magnetfeldstärken und nicht zu hohen Massenstromdichten die Kühlung schneller ist als der Temperaturangleich zwischen Ionen und Elektronen. Damit ist für den vorgesehenen Gültigkeitsbereich der Näherungslösung (hohe Magnetfeldstärken) eine Beschreibung des Plasmas als Ein-Teilchen-Fluid nur als sehr grobe Näherung akzeptabel.

In diesem Abschnitt soll ein Vergleich zwischen der analytischen Näherungslösung des Ein-Teilchen-Modells, wie sie in Kapitel 2.2 angegeben ist, mit den Ergebnissen aus der iterativen Lösung der Zwei-Teilchen-Beschreibung durchgeführt werden. Dazu wurden die Parameter Magnetfeldstärke $B$ und Massenstromdichte $\dot{m}$ so gewählt, daß der Einfluß der Zyklotronstrahlung auf die Kühlung der Emissionsregion ständig zunimmt. Die Masse des weißen Zwergs wurde dabei konstant gehalten $\left(M_{\mathrm{wd}}=0,6 M_{\odot}\right)$, da sie die Schicht wie die Massenstromdichte beeinflußt und an den grundsätzlichen Aussagen nichts ändert. Zur Umrechnung der Intensitäten aus der Strahlungstransportgleichung in Flüsse der Emissionsregion wurde wie in Abschnitt 3.4 .2 verfahren.

Eine Magnetfeldstärke von $B=30 \mathrm{MG}$ und $\dot{m}=100 \mathrm{~g} \mathrm{~s}^{-1} \mathrm{~cm}^{-2}$ für die Massenstromdichte ergeben ein Verhältnis von Äquipartitionszeit zu Zyklotronkühlzeit $t_{\mathrm{ei}} / t_{\mathrm{cyc}} \approx$ $10^{-2}<1$ (siehe S. 9), so daß nach der einfachen Abschätzung der Zyklotronverluste als optisch dicke Strahlung eine Unterscheidung der Temperaturen von Ionen und Elektronen nicht notwendig ist. Auch der zu diesem Magnetfeld und Massenfluß berechnete Wert $\eta_{\text {sh }}=6,87 \cdot 10^{-3}$, des bei der Herleitung der analytischen Ein-Teilchen-Lösung eingeführten Parameters $\eta_{\mathrm{sh}}$ (siehe S. 25), dem Verhältnis von Bremsstrahlungskühlzeit zu 


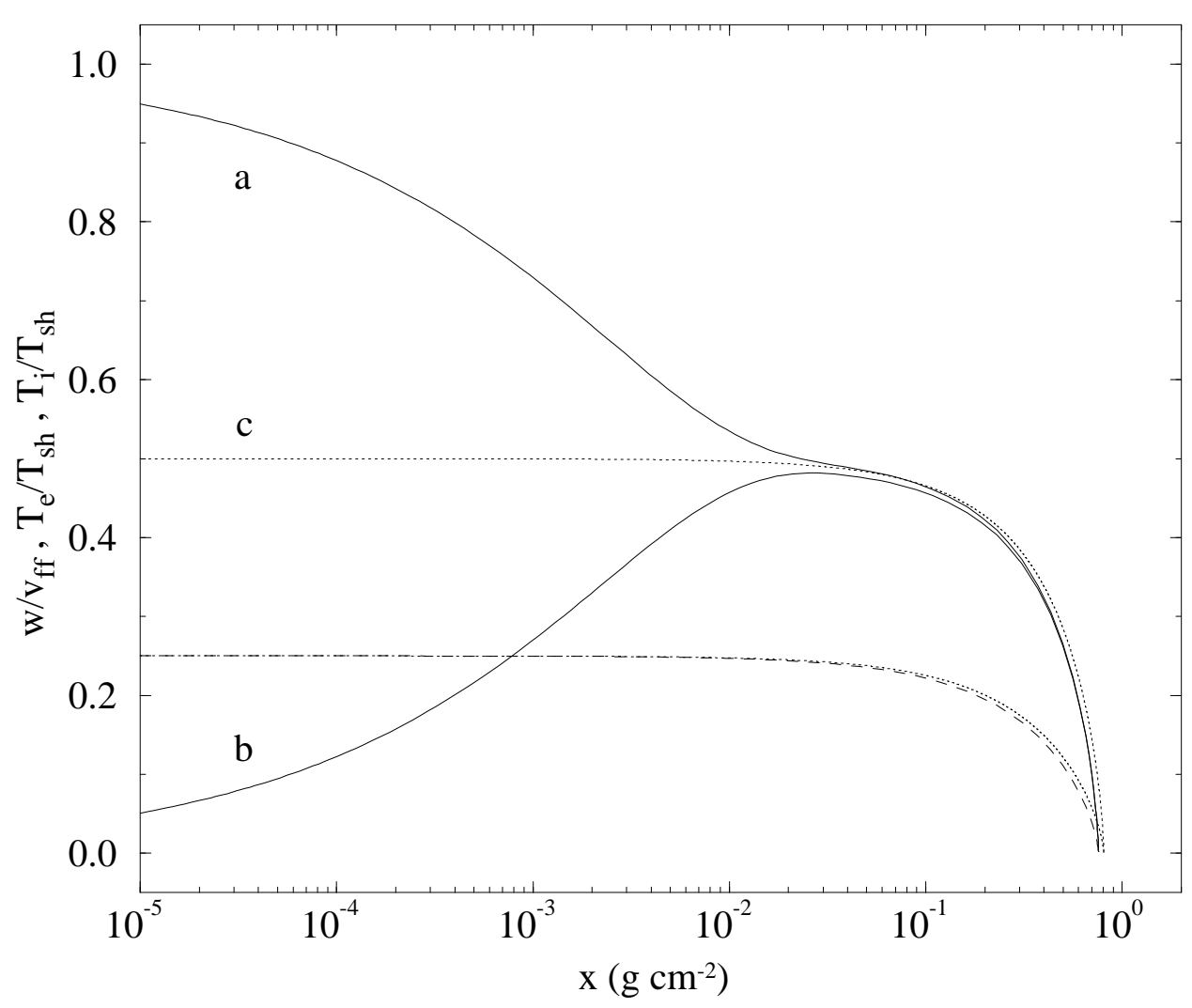

Abbildung 3.10: Vergleich des Temperatur- und Geschwindigkeitsverlaufs eines Ein-Teilchen-Plasmas mit dem eines Zwei-Teilchen-Plasmas bei Bremsstrahlungsund Zyklotronstrahlungskühlung zu $M_{\mathrm{wd}}=0,6 M_{\odot}, \dot{m}=100 \mathrm{~g} \mathrm{~s}^{-1} \mathrm{~cm}^{-2}$ und $B=30$ MG. (a) normierte Ionen-, (b) Elektronentemperatur $\left(T_{\mathrm{sh}}:=3 m_{\mathrm{p}} v_{\mathrm{ff}}^{2} / 16 k_{\mathrm{B}}\right)$ und normierte Geschwindigkeit (gestrichelte Linie) der Zwei-Teilchen-Rechnung. Die Ergebnisse der Ein-Teilchen-Rechnungen sind als gepunktete Linien dargestellt: (c) normierte Elektronentemperatur und Geschwindigkeit.

Zyklotronkühlzeit am Stoß, legt nahe, daß eine bremsstrahlungsdominierte Schichtung erzeugt wird und damit das Ein-Teilchen-Modell gerechtfertigt ist. In Abbildung 3.10 sind nun die resultierenden Verläufe von Geschwindigkeit und Temperatur aus dem EinTeilchen- bzw. Zwei-Teilchen-Modell dargestellt. Die Struktur zeigt das schon bekannte Verhalten der Kühlung vom Maximalwert am Anfang der Schicht bzw. die Äquipartition der Teilchentemperaturen mit folgender Kühlung. Die starke Ähnlichkeit mit den Ergebnissen für reine Bremsstrahlung in Abbildung 3.8 bestätigt die erwartete Dominanz dieses Prozesses. Dennoch sorgt die Zyklotronkühlung während der Äquipartition in der Zwei-Teilchen-Beschreibung für eine im Vergleich zum Ein-Teilchen-Modell leicht geringere Maximaltemperatur der Elektronen, was sich ebenso in einer weniger ausgedehnten geheizten Schicht ausdrückt.

Die Differenzen der Strukturen der Emissionsregion spiegeln sich in den zugehörigen Spektren wider, die in Abbildung 3.11 aufgetragen sind. Dort ist der Anteil der optisch dünnen Bremsstrahlung $\left(\nu>10^{15} \mathrm{~Hz}\right)$ klar zu erkennen, der in beiden Model- 


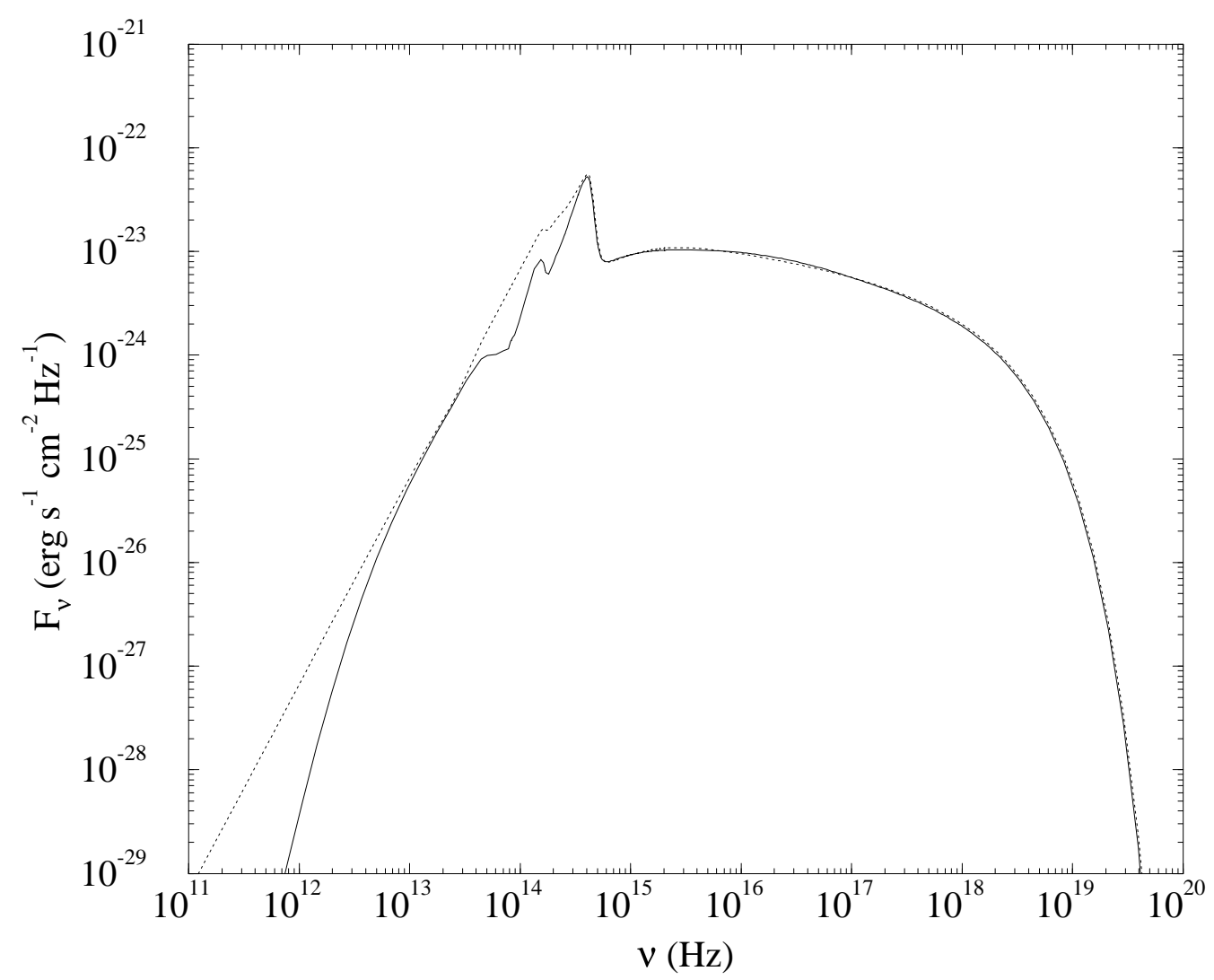

Abbildung 3.11: Spektrum der Emissionsregion zu den Schichtungen aus Abbildung 3.10. Dargestellt ist der spektrale Fluß $F_{\nu}\left(\vartheta=5^{\circ}\right)$ gegen die Frequenz $\nu$ der Zwei-Teilchen- (durchgezogende Linie) und der Ein-Teilchen-Rechnung (gepunktete Linie).

len das Spektrum, und damit den Energieinhalt des Strahlungsflusses, bestimmt. Der leicht höhere Fluß aus der Ein-Teilchen-Beschreibung wird durch die etwas größere Temperatur und weiter ausgedehnte Schicht verursacht. Auch der optisch dicke Bereich der Bremsstrahlung weist eine starke Ähnlichkeit mit den schon im Fall der reinen Bremsstrahlungskühlung gefundenen Spektren auf: Der Fluß aus dem Ein-Teilchen-Modell zeigt den Verlauf eines isothermen Schwarzkörpers zur Temperatur $T_{\mathrm{sh}} / 2$, während das Spektrum der Zwei-Teilchen-Beschreibung durch die ansteigende Elektronentemperatur erzeugt wird. Die optisch dünnen bzw. dicken Teile der schwachen Komponente der Zyklotronstrahlung $\left(2 \cdot 10^{13} \mathrm{~Hz}<\nu<6 \cdot 10^{14} \mathrm{~Hz}\right)$ werden in ähnlicher Weise gebildet. Die Temperaturstruktur am Anfang der geheizten Schicht verursacht die zuvor (siehe Kap. 3.4.2) beschriebene Absorption der Harmonischen, die bei den hohen Temperaturen und dem kleinen Sichtwinkel zum Magnetfeld stark verschmiert sind. Aus dem Ein-TeilchenModell kann eine solche Absorption nicht resultieren, denn eine optische Tiefe $\tau_{\nu}>1$ entsteht in den Anfangsbereichen der Schicht bei konstanter Temperatur $T_{\mathrm{sh}} / 2$. Daher wird die resultierende Zyklotronstrahlung im optisch dicken Bereich durch den Fluß des 


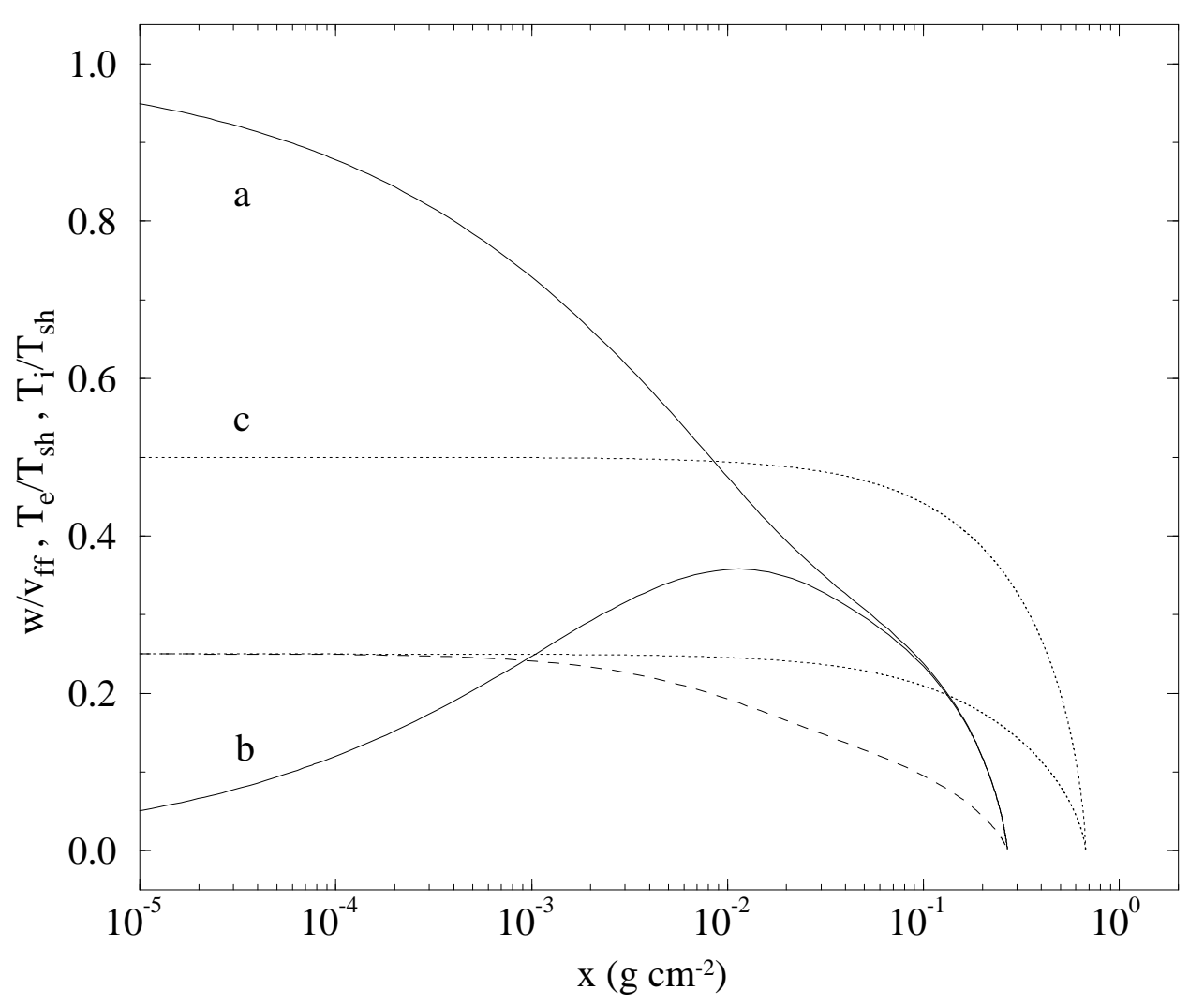

Abbildung 3.12: Darstellung der Schichtung der Emissionsregion wie in Abbildung 3.10, aber mit geringerer Massenstromdichte $\dot{m}=1 \mathrm{~g} \mathrm{~s}^{-1} \mathrm{~cm}^{-2}$.

Schwarzkörpers zu dieser Temperatur gebildet円. Im optisch dünnen Bereich unterscheiden sich die beiden Modelle dagegen nicht mehr stark: Hier gilt, wie im Bereich der optisch dünnen Bremsstrahlung, daß die leicht höhere Temperatur und weiter ausgedehnte Schicht des Ein-Teilchen-Modells einen geringfügig größeren Fluß verursacht; die Form der Spektren beider Modelle ist aber ähnlich. Die analytische Näherung liefert somit bei dominanter Bremsstrahlung eine gute Darstellung des harten Röntgenflusses der geheizten Region, ergibt aber im Bereich der Zyklotronstrahlung und optisch dicken Bremsstrahlung deutliche Unterschiede zu der Zwei-Teilchen-Beschreibung des Akkretionsplasmas.

${ }^{1} \mathrm{Daß}$ das Spektrum bei $\nu \approx 1 \cdot 10^{14} \mathrm{~Hz}$ eine Einsenkung zeigt, liegt in den Polarisationseigenschaften der Zyklotronstrahlung begründet. Um diese zu beschreiben, wird die Intensität in zwei Komponenten aufgeteilt, den sog. „ordentlichen“ und „außerordentlichen“ Strahl. Da nicht näher auf die Polarisation eingegangen werden wird, soll hier nur erwähnt werden, daß die Emissivität und damit der Absorptionskoeffizient (im LTE) des außerordentlichen Strahls sehr viel größer ist als der des ordentlichen (siehe z. B. Bekefi (1966), Ramaty (1969) oder Chanmugam \& Dulk (1981)). Diese Komponente erreicht bei gleicher Temperatur daher eher optische Tiefen $\tau_{\nu}>1$ als die ordentliche und dominiert die Intensität im optisch dünnen Teil. Das resultierende Spektrum entsteht nun durch Überlagerung der beiden Strahlen, was zu der angegebenen Einsenkung führt, da der ordentliche Strahl oberhalb dieser Frequenz optisch dünn wird und die rasch abfallende Emissivität kaum beiträgt. Bis auch der außerordentliche Strahl optisch dünn wird $\left(\nu>4 \cdot 10^{14} \mathrm{~Hz}\right)$, entspricht dem dargestellten Fluß daher die Schwarzkörperstrahlung einer Polarisationsmode. 


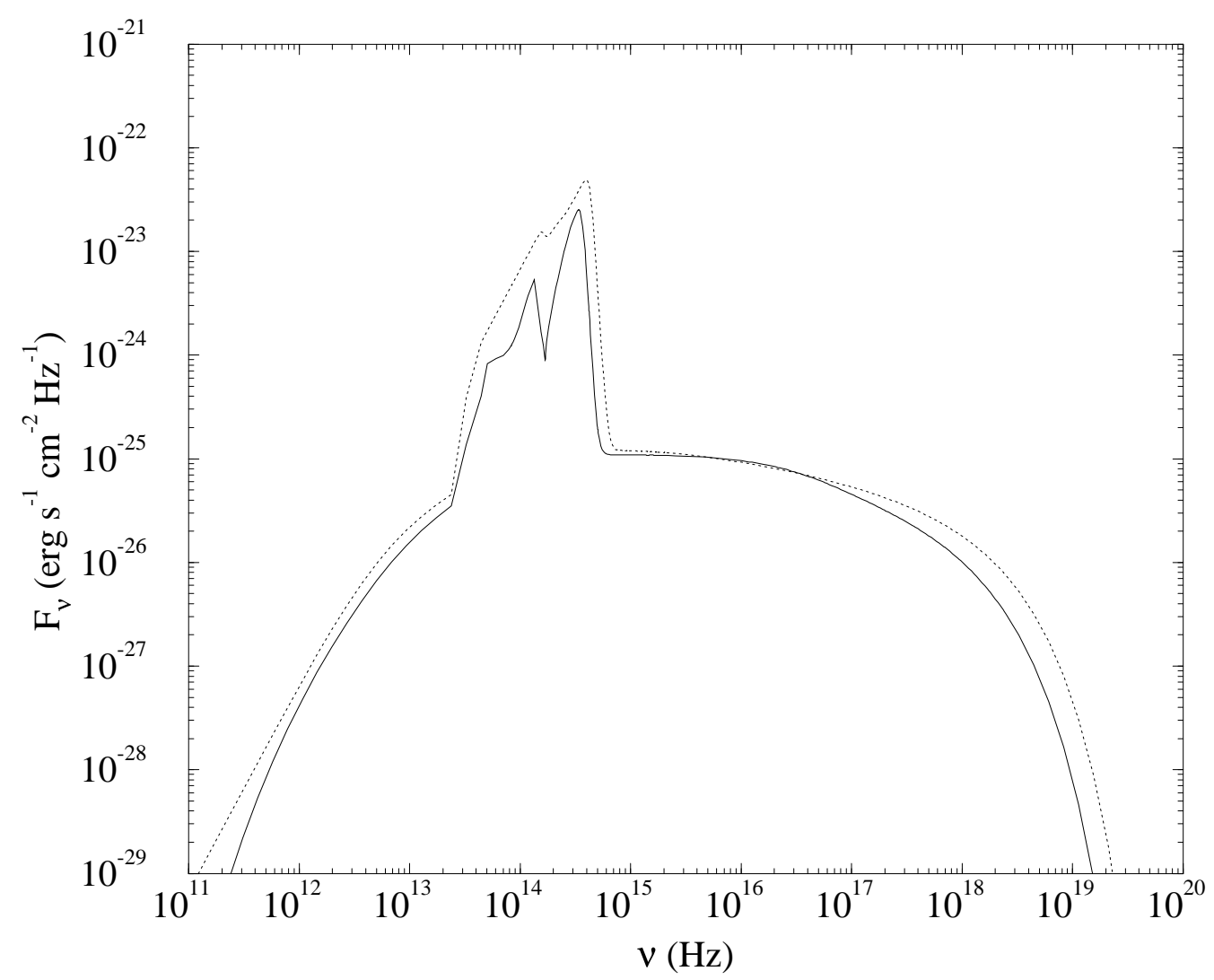

Abbildung 3.13: Darstellung des Spektrums der Emissionsregion wie in Abbildung 3.11, aber zu den Schichtungen mit $\dot{m}=1 \mathrm{~g} \mathrm{~s}^{-1} \mathrm{~cm}^{-2}$ aus Abbildung 3.12.

Eine Verringerung der Massenstromdichte vergrößert das Verhältnis von Äquipartitionszeit zu Zyklotronkühlzeit; bei einem Magnetfeld von $B=30 \mathrm{MG}$ und einer Massenstromdichte von $\dot{m}=1 \mathrm{~g} \mathrm{~s}^{-1} \mathrm{~cm}^{-2}$ gilt $t_{\mathrm{ei}} / t_{\mathrm{cyc}} \approx 3>1$. Die Zyklotronstrahlung ist hier effektiv genug, so daß eine Kühlung des Plasmas stattfindet, bevor der Temperaturausgleich zwischen den Teilchen beendet ist. Die Annahme einer gemeinsamen Temperatur der Ionen und Elektronen ist nicht mehr über den gesamten Bereich der Emissionsregion gerechtfertigt; eine Zwei-Teilchen-Beschreibung ist erforderlich. In der analytischen Ein-Teilchen-Lösung bewirkt die nun effektiver auftretende Zyklotronstrahlung eine Erhöhung des Verhältnisses der Bremsstrahlungskühlzeit zur Zyklotronkühlzeit; für obige Magnetfeldstärke und Massenstromdichte findet man: $\eta_{\text {sh }}=0,857$. Der im Vergleich zur höheren Massenstromdichte stärkere Einfluß der Zyklotronkühlung führt nun in beiden Modellen zu einer Verringerung der Ausdehnung der geheizten Schicht; die resultierenden Temperatur- und Geschwindigkeitsverläufe sind in Abbildung 3.12 aufgetragen. Wegen der erhöhten Zyklotronstrahlung kann das Plasma in der Zwei-Teilchen-Beschreibung schon während der Phase des Temperaturangleichs von Ionen und Elektronen kühlen. Die Maximaltemperatur der Elektronen ist daher deutlich geringer als im Fall der höheren Massenstromdichte in Abbildung 3.10. Im Ein-Teilchen-Modell wird die stärkere Kühlung nur durch den höheren Wert von $\eta_{\mathrm{sh}}$ beschrieben; der Strahlungsverlustterm in der zu- 


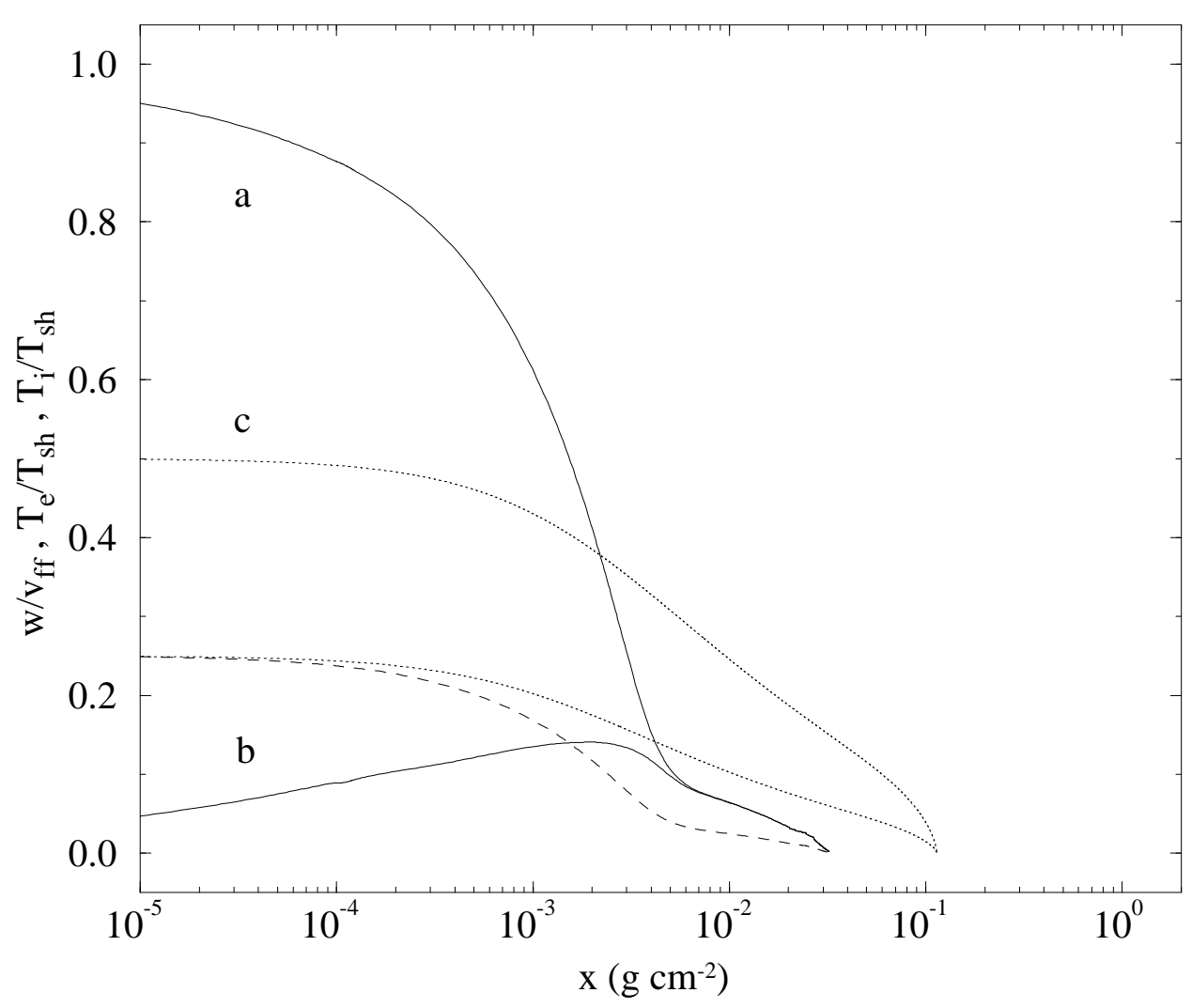

Abbildung 3.14: Darstellung der Schichtung der Emissionsregion wie in Abbildung 3.12, aber mit erhöhter Kühlung durch Zyklotronstrahlung zu $B=100 \mathrm{MG}$.

gehörigen Energiegleichung (Gl. 2.11) ist damit proportional zu $\rho^{2} T^{1 / 2}+3 \cdot 10^{-32} \rho^{0,15} T^{2,5}$. Somit überwiegt die Dichteabhängigkeit der Bremsstrahlungskomponente die Temperaturabhängigkeit des Zyklotronanteils, und die Kühlung von der Anfangstemperatur $T_{\mathrm{sh}} / 2$ setzt erst bei größeren Dichten ein als bei der Zwei-Teilchen-Beschreibung. Die resultierende Temperaturstruktur ist demgemäß auch ähnlich der bei der bremsstrahlungdominierten Kühlung gefundenen Lösung, die in Abbildung 3.10 dargestellt ist. Das Ein-TeilchenModell unterschätzt also die Zyklotronkühlung am Anfang der Emissionsregion und ergibt Strukturen, die den Einfluß des Magnetfeldes unzureichend wiedergeben.

Abbildung 3.13 (vorherige Seite) stellt die Spektren der Emissionsregion dar, die den Einfluß der Zyklotronstrahlung, durch die Erhöhung des Verhältnisses von Zyklotronfluß zu Bremsstrahlungsfluß, verdeutlichen. Der Zyklotronanteil des Zwei-Teilchen-Spektrums zeigt die Absorption der Harmonischen im optisch dicken Bereich, wobei die Einsenkungen schärfer sind als bei der hohen Massenstromdichte in Abbildung 3.10, da die Temperaturen hier geringer sind und die Harmonischen dadurch weniger verschmieren. Aus dem gleichen Grund ist der optisch dünne Fluß dieser Komponente verringert. Der Zyklotronanteil aus der Ein-Teilchen-Beschreibung verändert sich im Vergleich zur hohen Massenstromdichte dagegen nur wenig: Die ähnlichen Temperaturschichtungen erzeugen ähnliche Spektren. Insbesondere ist der optisch dicke Anteil weiterhin durch den Schwarzkörperfluß zu $T_{\mathrm{sh}} / 2$ 


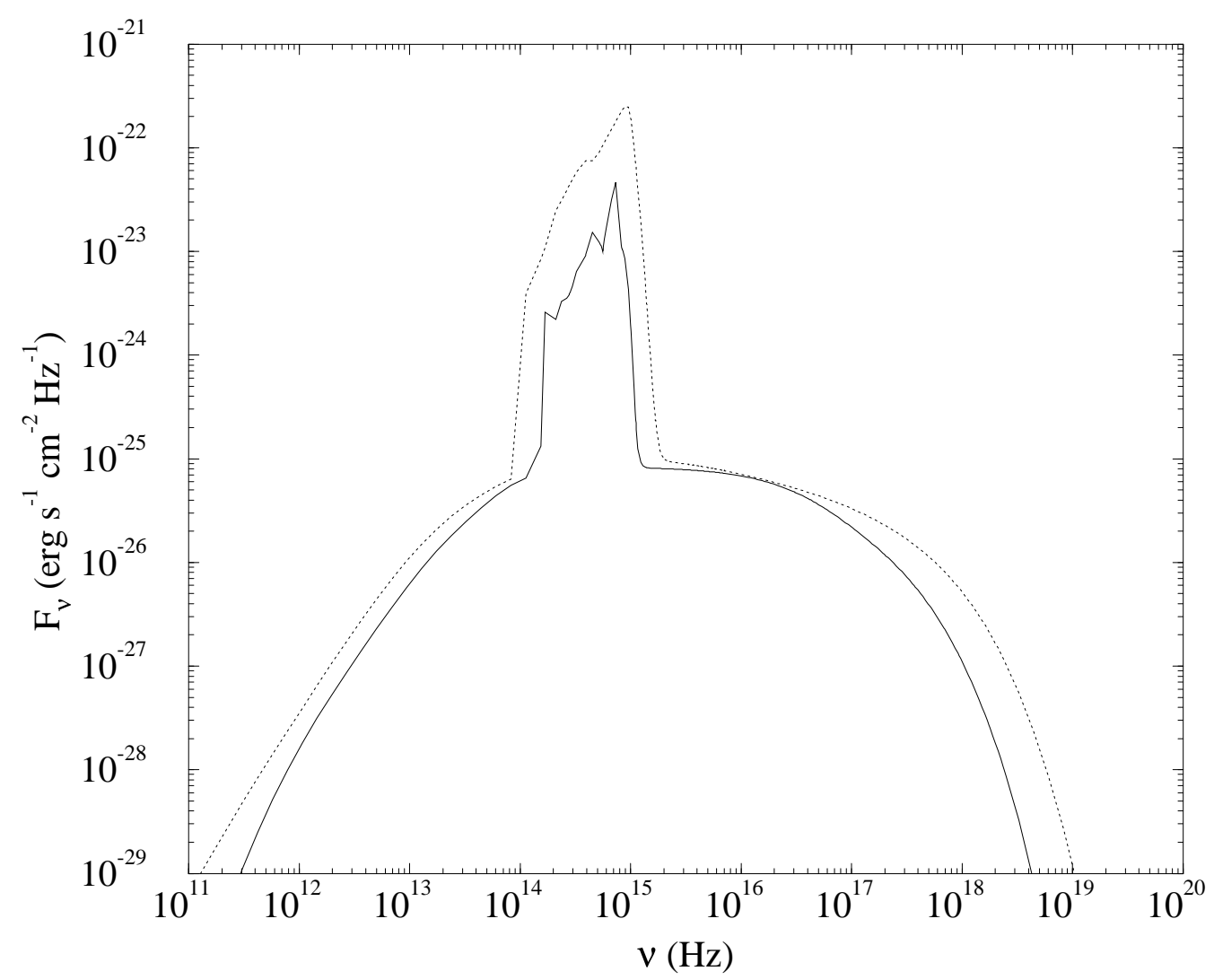

Abbildung 3.15: Darstellung des Spektrums der Emissionsregion wie in Abbildung 3.13, aber zu den Schichtungen eines Magnetfeldes mit $B=100 \mathrm{MG}$ aus Abbildung 3.14.

begrenzt. Im Bereich der optisch dünnen Bremsstrahlung ist der Fluß in beiden Modellen deutlich kleiner als bei der hohen Massenstromdichte, und der Röntgenfluß bei hohen Frequenzen ist wegen der geringeren Temperaturen bei hohen Dichten ebenso reduziert. Die Unterschiede in der Temperaturstruktur der beiden Modelle finden sich auch hier wieder: Das Bremsstrahlungsspektrum aus dem Ein-Teilchen-Modell ist - wegen der im Vergleich zum Zwei-Teilchen-Modell höheren Temperaturen der Emissionsregion - deutlich „härter", d. h. der Fluß bei hohen Frequenzen ist größer.

Um den Einfluß der Zyklotronstrahlung weiter zu erhöhen, wurde bei der Massenstromdichte $\dot{m}=1 \mathrm{~g} \mathrm{~s}^{-1} \mathrm{~cm}^{-2}$ die Magnetfeldstärke auf $B=100 \mathrm{MG}$ erhöht. Bei diesem Parametersatz ist $t_{\text {ei }} / t_{\text {cyc }} \approx 2000 \gg 1$, so daß eine Zwei-Teilchen-Beschreibung unumgänglich ist. Der Vergleich mit dem Ein-Teilchen-Modell mit dem resultierenden Wert $\eta_{\mathrm{sh}}=274$ soll hier dennoch durchgeführt werden, da sich erst bei so hohen Parametern eine ähnlich deutliche Änderung der Temperaturstruktur einstellt, wie sie bei der Zwei-Teilchen-Beschreibung schon früher auftritt. In Abbildung 3.14 sind die Resultate der beiden Modelle für die Struktur der Emissionsregion gezeigt. Der Temperaturund Geschwindigkeitsverlauf aus der Zwei-Teilchen-Rechnung entspricht dem erwarteten Verhalten bei einer Verstärkung der Kühlung. Dabei ist die Maximaltemperatur der Elektronen nun extrem reduziert, und die Ausdehnung beträgt nur noch $\approx 1 / 30$ der geheizten 
Schicht nach reiner Bremsstrahlung. Auch im Ein-Teilchen-Modell wird nun eine deutliche Abweichung des Temperaturverlaufs von der bremsstrahlungsdominierten Form sichtbar. Für den hohen Wert von $\eta_{\text {sh }}$ kommt jetzt der Anteil der Zyklotronstrahlung am Energieverlustterm zum tragen $\left(\rho^{2} T^{1 / 2}+9 \cdot 10^{-30} \rho^{0,15} T^{2,5}\right)$ und reduziert die geheizte Schicht auf $1 / 10$ der Ausdehnung für $\eta_{\mathrm{sh}} \ll 1$. Die zugehörigen Spektren finden sich in Abbildung 3.15 (vorherige Seite) und zeigen erneut die oben beschriebenen Eigenschaften. Allerdings sind nun die Abweichungen zwischen den beiden Modellen bezüglich der auftretenden Temperaturen der Schichtungen so groß, daß sowohl die Zyklotronstrahlung als auch die harte Bremsstrahlung wesentlich unterschiedliche Flüsse liefern.

\subsubsection{Bewertung}

Der Vergleich der Ergebnisse aus dem Zwei-Teilchen-Modell mit der analytischen Näherungslösung der Ein-Teilchen-Beschreibung zeigt deutlich die Einschränkungen letzteren Zugangs. In Kapitel 3.5.1 konnte dargestellt werden, daß im Bereich der dominanten Bremsstrahlungskühlung beide Modelle übereinstimmende Resultate ergeben und damit äquivalent sind.

Die Berücksichtigung der Zyklotronstrahlung in der Ein-Teilchen-Beschreibung (Kap. 3.5.2) erfaßt jedoch nur unvollständig deren Einfluß auf die Emissionsregion. So kann bei vorhandenem Magnetfeld nicht mehr davon ausgegangen werden, daß die Elektronen die gleiche Temperatur wie die Ionen besitzen und damit ebenfalls auf die Stoßtemperatur geheizt werden. Auch wenn die Kühlung durch Zyklotronstrahlung über den Parameter $\eta_{\text {sh }}$ richtig erfaßt wäre, fände sich kein Wert dafür, der die Temperatur am Anfang verringerte. Die mit einer solchen Temperaturverteilung berechneten Spektren zeigen im optisch dicken Bereich daher immer einen Verlauf, der der Schwarzkörperstrahlung zu der Stoßtemperatur entspricht und liefern somit u. U. zuviel Fluß.

Darüberhinaus ist fraglich, ob die Zyklotronverluste, über die verwendete Näherung als optisch dicker Fluß, richtig bestimmt sind, denn die optisch dicken Teile der Zyklotronspektren aus der Zwei-Teilchen-Beschreibung weichen von einem Schwarzkörperspektrum wegen der Absorption in den Harmonischen zum Teil erheblich ab.

Die vorgestellte Ein-Teilchen-Beschreibung liefert zwar eine leicht zu handhabende Formel für die Struktur der Emissionsregion, die die qualitativen Auswirkungen zusätzlicher Zyklotronkühlung korrekt erfaßt und damit Veränderungen in der Bremsstrahlung erzeugt, die diesem Energieverlust entsprechen. Jedoch ist die Form des Bremsstrahlungsspektrums von der Temperaturstruktur abhängig und daher in den beiden beschriebenen Modellen unterschiedlich. Weiterhin lassen die Defizite in der Darstellung der Zyklotronstrahlung und im Absolutwert des Flusses über den gesamten Spektralbereich eine quantitative Untersuchung beobachteter Spektren der Polare mit dem gewählten Zugang ungeeignet erscheinen. 


\subsection{Auswirkungen der genäherten Geometrie}

\subsubsection{Schichtungen großer Höhe}

Bei der Herleitung des hydrodynamischen Gleichungssystems (Gln. 3.16 bis 3.19) wurde angenommen, daß parallele Magnetfeldlinien in der Akkretionsregion senkrecht auf der Sternoberfläche stehen und das Plasma daher als eindimensionale Strömung konstanter Querschnittsfläche $A_{\text {acc }}$ mit höhenunabhängigem $\dot{m}:=\dot{M} / A_{\text {acc }}=$ const zu beschreiben ist (siehe Kap. 3.2.1). Diese Näherung ist allerdings nur gerechtfertigt, wenn die Höhe der Emissionsregion über der Oberfläche des weißen Zwergs wesentlich kleiner ist als dessen Radius $\left(h_{\mathrm{sh}} \ll R_{\mathrm{wd}}\right)$. Bei der Berechnung der Schichtungen zu verschiedenen Kombinationen der Parameter Masse $M_{\mathrm{wd}}$, Massenstromdichte $\dot{m}$ und Magnetfeldstärke $B$ zeigte sich jedoch, daß die Ausdehnung der geheizten Region bei einer nur schwachen Strahlungskühlung diese Bedingung nicht mehr erfüllt. So ergibt sich zu einer Masse $M_{\text {wd }}=0,6 M_{\odot}$ und Massenstromdichte $\dot{m}=10^{-2} \mathrm{~g} \mathrm{~s}^{-1} \mathrm{~cm}^{-2}$ zum Magnetfeld $B=10 \mathrm{MG}$ eine Höhe $h_{\mathrm{sh}} \approx 16 \% R_{\mathrm{wd}}$.

In diesem Kapitel soll untersucht werden, inwieweit die durchgeführte Näherung eine Schichtung beeinflußt, deren Höhe im Vergleich zum Sternradius nicht mehr zu vernachlässigen ist. Dafür wird - als einfachstes Modell einer höhenabhängigen Massenstromdichte - der Einfall mit konstanter Akkretionsrate $\dot{M}$ entlang radialer Linien auf einen Bruchteil $f$ der Sternoberfläche betrachtet. Bei einer kreisförmigen Akkretionsfläche auf dem weißen Zwerg entspricht der Emissionsregion somit in etwa ein Kegelstumpf, dessen Querschnitt nach außen hin zunimmt. Das realistischere Modell der Akkretion entlang konvergierender Dipolfeldlinien erfordert zusätzliche Parameter und schränkt daher den prinzipiellen Charakter der Untersuchung ein. Weiterhin soll als Strahlungsverlustprozeß allein Bremsstrahlung betrachtet werden, da der Kühlprozeß nur die Höhe der Emissionsregion bestimmt und keine Auswirkungen auf die Geometrie der Akkretion hat.

Das hydrodynamische Gleichungssystem (Gln. 3.16 bis 3.19) wurde in Kugelkoordinaten umgeschrieben $(\theta, \phi$-Abhängigkeiten vernachlässigt) und in der Energiegleichung der Elektronen (Gl. 3.19) der Strahlungsverlustterm durch optisch dünne Bremsstrahlung ersetzt; die Strahlungstransportgleichung (Gl. 3.23) fällt in diesem Zusammenhang fort. Die Form der Energiegleichungen ändert sich nur insoweit, daß die Differentiation nach der kartesiche Koordinate $z$ durch die nach der radialen $r$ ersetzt wird. Dagegen geht in die Kontinuitätsgleichung (Gl. 3.16) die Koordinate $r$ durch die Höhenabhängigkeit der Akkretionsfläche ein, und in der Impulsgleichung (Gl. 3.17) muß die Konstanz der Gravitationsbeschleunigung aufgegeben werden. Dies führt auf folgende Form:

$$
\begin{aligned}
\rho w & =\frac{\dot{M}}{4 \pi r^{2} f}=: \dot{m}(r) \\
w \frac{\mathrm{d} w}{\mathrm{~d} r}+\frac{1}{\rho} \frac{\mathrm{d}}{\mathrm{d} r}\left(P_{\mathrm{e}}+P_{\mathrm{i}}\right) & =-\frac{G M_{\mathrm{wd}}}{r^{2}} .
\end{aligned}
$$

Das neue Gleichungssystem wurde nach der in Kapitel 3.3 beschrieben Methode gelöst. Wegen der expliziten Abhängigkeit der Gleichungen von der radialen Koordinate $r$ muß 


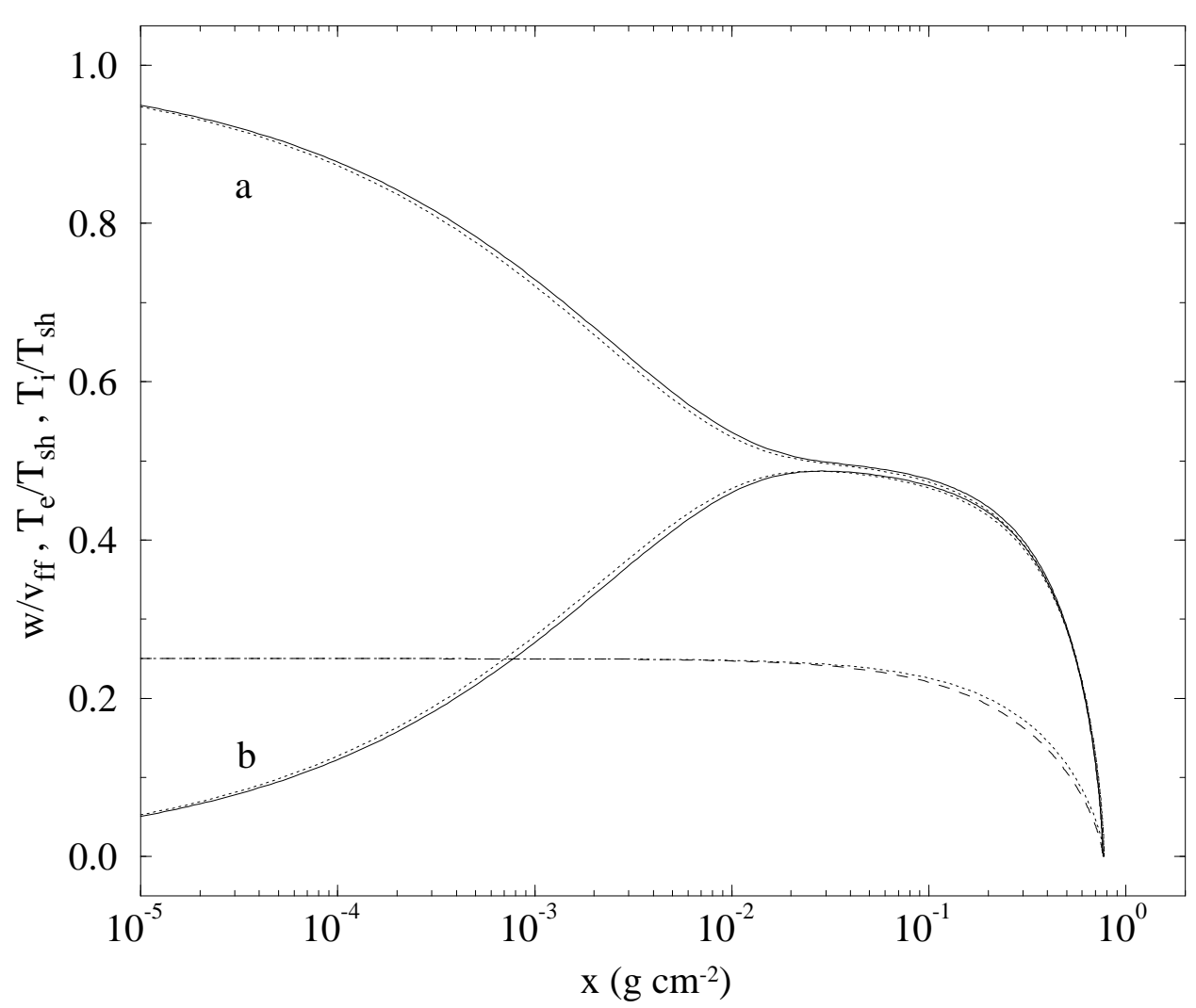

Abbildung 3.16: Vergleich des Temperatur- und Geschwindigkeitsverlaufs einer Akkretionsregion konstanter Massenstromdichte, mit dem eines höhenabhängigen $\dot{m}$, zu den Parametern $M_{\mathrm{wd}}=0,6 M_{\odot}, \dot{m}=1 \mathrm{~g} \mathrm{~s}^{-1} \mathrm{~cm}^{-2}$ bei reiner Bremsstrahlung. (a) normierte Ionen-, (b) Elektronentemperatur $\left(T_{\mathrm{sh}}:=3 m_{\mathrm{p}} v_{\mathrm{ff}}^{2} / 16 k_{\mathrm{B}}\right)$ und normierte Geschwindigkeit (gestrichelte Linie) der Rechnung mit $\dot{m}=$ const. Die Ergebnisse aus der Rechnung mit $\dot{m}(r)$ sind als gepunktete Linien dargestellt. Die Normierungen erfolgten mit $v_{\mathrm{ff}}\left(R_{\mathrm{wd}}\right)$ bei konstater Massenstromdichte bzw. mit $v_{\mathrm{ff}}\left(h_{\mathrm{sh}}\right)$ bei $\dot{m}(r)$.

der Ort des Stoßes über der Sternoberfläche jedoch iterativ bestimmt werden, damit die Randbedingung $w \approx 0$ am Ende der durch die Kühlung festgelegten Ausdehnung der Emissionsregion mit dem Ort $r=R_{\mathrm{wd}}$ übereinstimmt.

Bei konstanter Massenstromdichte wird die Dichtestruktur der Emissionsregion allein durch die Abbremsung des Stroms auf der Sternoberfläche gebildet; die Kontinuitätsgleichung (Gl. 3.16) beschreibt die Zunahme der Dichte bei abnehmender Geschwindigkeit. Im Fall der Akkretion entlang radialer Magnetfeldlinien kommt weiterhin eine Verringerung der Querschnittsfläche des Plasmas vom Stoß zur Sternoberfläche hinzu. Die zugehörige Kontinuitätsgleichung (Gl. 3.35) beschreibt diesen Vorgang als Erhöhung der Massenstromdichte mit abnehmender radialer Koordinate. Damit wird die Dichte in der geheizten Region zusätzlich erhöht und erreicht einen größeren Wert als bei gleicher geometrischer Höhe im Fall der konstanten Massenstromdichte. Solch eine Dichtezunahme sorgt wiederum für ein Anwachsen der Bremstrahlungskühlung in der Emissionsregion, 


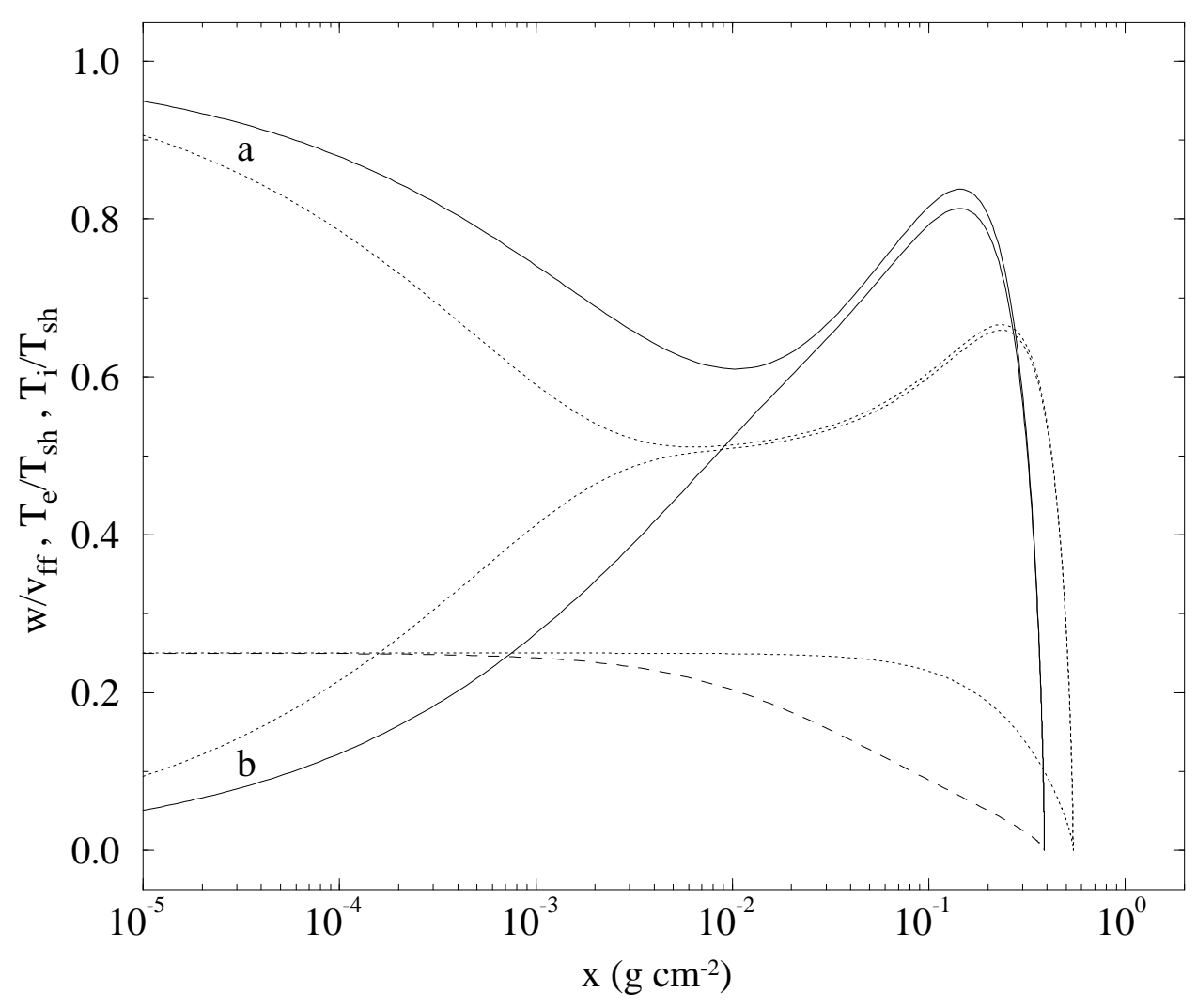

Abbildung 3.17: Darstellung des Temperatur- und Geschwindigkeitsverlaufs wie in Abbildung 3.16, aber für eine kleinere Massenstromdichte $\dot{m}=10^{-2} \mathrm{~g} \mathrm{~s}^{-1} \mathrm{~cm}^{-2}$.

und die geheizte Schicht wird im Vergleich zu einer Akkretionsregion mit $\dot{m}=$ const weniger weit ausgedehnt sein. Dieser Unterschied zeigt sich besonders bei Parametern für $M_{\text {wd }}$ und $\dot{m}$, die eine Stoßhöhe bewirken, die im Vergleich zum Radius des weißen Zwergs nicht klein ist.

In der Abbildung 3.16 sind die resultierenden Temperatur- und Geschwindigkeitsstrukturen aus Rechnungen $\left(M_{\mathrm{wd}}=0,6 M_{\odot}\right)$ mit konstanter bzw. höhenabhängiger Massenstromdichte zu $\dot{m}=1 \mathrm{~g} \mathrm{~s}^{-1} \mathrm{~cm}^{-2}$ verglichen, wobei im Fall $\dot{m}(r)$ dieser Wert am Stoß erreicht wird. Die Schichtungen ergeben nur geringe Abweichungen zwischen den beiden Zugängen: Die Stoßhöhe bei $\dot{m}=1 \mathrm{~g} \mathrm{~s}^{-1} \mathrm{~cm}^{-2}=$ const beträgt $h_{\mathrm{sh}}=5,2 \cdot 10^{-2} R_{\mathrm{wd}}$, während sie für $\dot{m} \sim 1 \mathrm{~g} \mathrm{~s}^{-1} \mathrm{~cm}^{-2} / r^{2}$ den Wert $h_{\mathrm{sh}}=5,1 \cdot 10^{-2} R_{\mathrm{wd}}$ annimmt. Auch die erreichten Maximaltemperaturen der Elektronen unterscheiden sich kaum; lediglich die Form der Kurven weicht leicht voneinander ab. Rechnungen mit höheren $\dot{m}$-Werten am Stoß zeigen darüberhinaus weit geringere Abweichungen: Für $h_{\mathrm{sh}}<10^{-2} R_{\mathrm{wd}}$ ist die Unterscheidung zwischen konstanter und höhenabhängiger Massenstromdichte unwesentlich.

Die Ergebnisse der Temperatur- und Geschwindigkeitsverläufe aus den Rechnungen zu $\dot{m}=10^{-2} \mathrm{~g} \mathrm{~s}^{-1} \mathrm{~cm}^{-2}$ sind in Abbildung 3.17 dargestellt. Hier sind die Unterschiede zwischen den beiden Fällen der Massenstromdichte erheblich. Die Stoßhöhe ist für die höhenabhängige Massenstromdichte zwar nur um $0,06 R_{\mathrm{wd}}$ geringer als für den kon- 
stanten Fall, aber die Maximaltemperaturen der Elektronen differieren um ca. 60\%: $T_{\max }=3,4 \cdot 10^{8} \mathrm{~K}$ bei $\dot{m}=$ const $\mathrm{zu} T_{\max }=1,2 \cdot 10^{8} \mathrm{~K}$ für die höhenabhängige Massenstromdichte. Der Grund dafür ist zum einen die schon erwähnte Erhöhung der Kühlung im Fall der höhenabhängigen Massenstromdichte. Wichtiger aber ist, daß hier die Stoßtemperatur $T_{\mathrm{sh}}\left(h_{\mathrm{sh}}\right)$ (Gl. 1.7) kleiner ist als im Fall $\dot{m}=$ const, denn die Frei-Fall-Geschwindigkeit nimmt für $h_{\mathrm{sh}}>R_{\mathrm{wd}}$ einen kleineren Wert an als $v_{\mathrm{ff}}\left(R_{\mathrm{wd}}\right)$. Darüberhinaus wirkt sich die Näherung $g=g\left(R_{\mathrm{wd}}\right)=$ const (siehe Kap. 3.2.1) im Gleichungssystem für die konstante Massenstromdichte aus. Werden die hydrodynamischen Gleichungen zu einer vorgegebenen Strahlungskühlung gelöst, ergibt sich eine Schichtung, in der der Strom auf der Sternoberfläche zur Ruhe kommt, wenn die gesamte akkretierte Energie $L_{\text {acc }}$ (Gl. 1.1) abgestrahlt wurde. Daraus resultiert die Höhe des Stoßes. Wird nun die Randbedingung $T_{\mathrm{sh}}\left(R_{\mathrm{wd}}\right)$ in der Höhe $h_{\mathrm{sh}}$ angenommen und $g=$ const gelassen, erfährt der Akkretionsstrom eine über die gesamte Höhe konstante Beschleunigung; das Plasma setzt eine zusätzliche potentielle Energie um, die ebenso abgestrahlt werden muß. Die Schichtung zu einem festen $\dot{m}$ wird daher weiter ausgedehnt und heißer sein, und die abgestrahlte Energie ist größer als im Fall $\dot{m}(r)$ mit $g(r)$ und angepassten Randbedingungen. Ein Vergleich zeigt, daß der Strahlungsfluß aus der Rechnung mit $\dot{m}(r)$ bei $\dot{m}=10^{-2} \mathrm{~g} \mathrm{~s}^{-1} \mathrm{~cm}^{-2}$ am Stoß, $F_{\text {rad }}=9,19 \cdot 10^{14} \mathrm{erg} \mathrm{s}^{-1} \mathrm{~cm}^{-2}$ beträgt und damit dem erwarteten Wert $L_{\text {acc }}\left(R_{\mathrm{wd}}\right) / A_{\text {acc }}$ entspricht, während das Ergebnis für den konstanten Fall $F_{\text {rad }}=2,1 \cdot 10^{15} \mathrm{erg} \mathrm{s}^{-1} \mathrm{~cm}^{-2}$ ist, entsprechend $L_{\mathrm{acc}}\left(R_{\mathrm{wd}}\right)\left(1+h_{\mathrm{sh}} / R_{\mathrm{wd}}\right) / A_{\mathrm{acc}}$. Bei hoch ausgedehnten Schichtungen, die unter Vernachlässigung der höhenabhängigen Terme gerechnet wurden, können die Abweichungen daher beträchtlich sein.

Analoge Rechnungen zu einer Masse $M_{\mathrm{wd}}=1,0 M_{\odot}$ bestätigen die obigen Ergebnisse, wobei die Abweichungen noch leicht erhöht werden. Die Auswirkungen der genäherten Geometrie auf die Maximaltemperatur der Elektronen in den Schichtungen zu den Massen zwischen $M_{\mathrm{wd}}=0,6 M_{\odot}$ und $1,0 M_{\odot}$ kann man nun derart zusammenfassen, daß für Parameter, die eine Stoßhöhe ergeben, die kleiner 1/100 des Sternradius ist, die Abweichung zu einer Lösung aus einer höhenabhängigen Rechnung vernachlässigbar ist. Bei $h_{\mathrm{sh}} \approx 10 \% R_{\mathrm{wd}}$ können die Differenzen bis zu $30 \%$ größer sein, im Gebiet $h_{\mathrm{sh}} \approx 1 R_{\mathrm{wd}}$ erreichen die Unterschiede bis zu $60 \%$ und nehmen dann weiter zu.

Obwohl in den Rechnungen nur Bremsstrahlung als Kühlprozeß berücksichtigt wurde, sind die Ergebnisse auch auf magnetische Regionen anwendbar, da Eigenschaften der Strahlung nicht explizit in die Betrachtungen eingeflossen sind. Weil Zyklotronstrahlung aber generell die Stoßhöhe herabsetzt, kann der zulässige $\dot{m}$-Bereich für eine feste Masse $M_{\text {wd }}$ vergrößert werden. Allerdings sind die Resultate nur grobe Richtwerte, die eine qualitative Bewertung einer gefundenen Schicht erlauben. Für quantitaive Aussagen muß ein genaueres Modell der Magnetfeldgeometrie und Höhenvariation der Massenstromdichte aufgestellt werden.

\subsubsection{Seitlich begrenzte Akkretionsregion}

Die Strahlungsverluste der Emissionsregion bestimmen entscheidend die Temperaturstruktur der geheizten Schicht. Um zu einem vorgegebenem Satz der Parameter Masse 


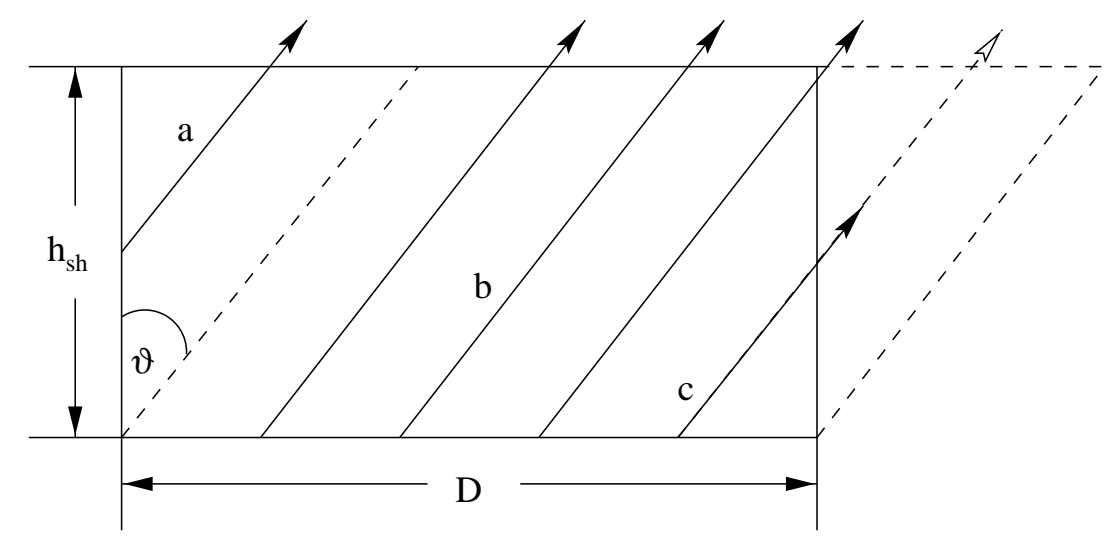

Abbildung 3.18: Zerlegung einer Emissionsregion der Höhe $h_{\text {sh }}$ und Breite $D$ in ein einzelne Strahlen, um optische-Tiefe-Effekte an den Seiten darzustellen. In Abhängigkeit des Sichtwinkels $\vartheta$ erreichen nicht alle Strahlen den oberen Rand der Region. Das gestrichelte Parallelogramm versinnbildlicht den Fall der „unendlich ausgedehnten" Schicht gleichen Volumens.

$M_{\text {wd }}$, Massenstromdichte $\dot{m}$ und Magnetfeldstärke $B$ den Energieverlust durch Brems- und Zyklotronstrahlung richtig zu erfassen, wird die Strahlungstransportgleichung (Gl. 3.23) mit einer vorgegeben hydrodynamischen Schichtung gelöst. Dabei geht die Annahme ein, daß die Akkretionsregion unendlich ausgedehnt ist, da seitliche Randeffekte vernachlässigt werden (siehe Kap. 3.3 und 3.4.2). Tatsächlich handelt es sich bei der emittierenden Region eines konkreten Polars aber um ein begrenztes Gebiet, dessen Ausdehnung $D$ auf der Sternoberfläche von den Systemparametern abhängt (siehe z. B. Lamb 1985; Beuermann, Stella \& Patterson 1987). Damit entspricht die Emissionsregion, je nach Verhältnis $D / h_{\mathrm{sh}}$, eher einer schmalen Säule bzw. einer dünnen Schicht (engl. „pill box-shaped“), deren Begrenzungen die Energieverhältnisse der geheizten Schicht beeinflußen können. Zur Beschreibung des Strahlungstransports in einem solchen endlichen Medium ist eine Umsetzung der zugehörigen Gleichung in mindestens zwei Raumdimensionen (bzw. drei Dimensionen bei fehlender Symmetrie) notwendig. Darüberhinaus erfordert dieser Zugang, um konsistente Lösungen zu liefern, eine ebensolche Darstellung der hydrodynamischen Gleichungen, und die allgemeine Form der Erhaltungssätze aus Kapitel 3.1 wäre gültig. Die Lösung dieses vollständigen Systems von Gleichungen ist bisher jedoch noch nicht gelungen und kann auch in dieser Arbeit nicht geleistet werden.

Die qualitativen Auswirkungen der Annahme der unendlich ausgedehnten Schicht auf deren Energieverhältnisse und die Spektren der Emissionsregion, sollen an Hand eines einfachen Modells, in das die Randeffekte bezüglich des Strahlungstransports eingehen, untersucht werden. Dazu wird die Grundfläche der Emissionsregion auf der Sternoberfläche als quadratisch angenommen (Kantenlänge $D$ ). Ihre Höhe ergibt sich aus der Stoßhöhe $h_{\text {sh }}$ der Schichtung zu den betrachteten Parametern $M_{\mathrm{wd}}, \dot{m}$ und $B$, die aus der Rechnung mit dem Strahlungstransport einer unendlich ausgedehnten Region resultierte. Diese Schichtung soll außerdem über die gesamte Breite der Emissionsregion gültig sein, so daß in horizontalen Schnitten zur Grundfläche die gleichen Werte der physikalischen Größen 


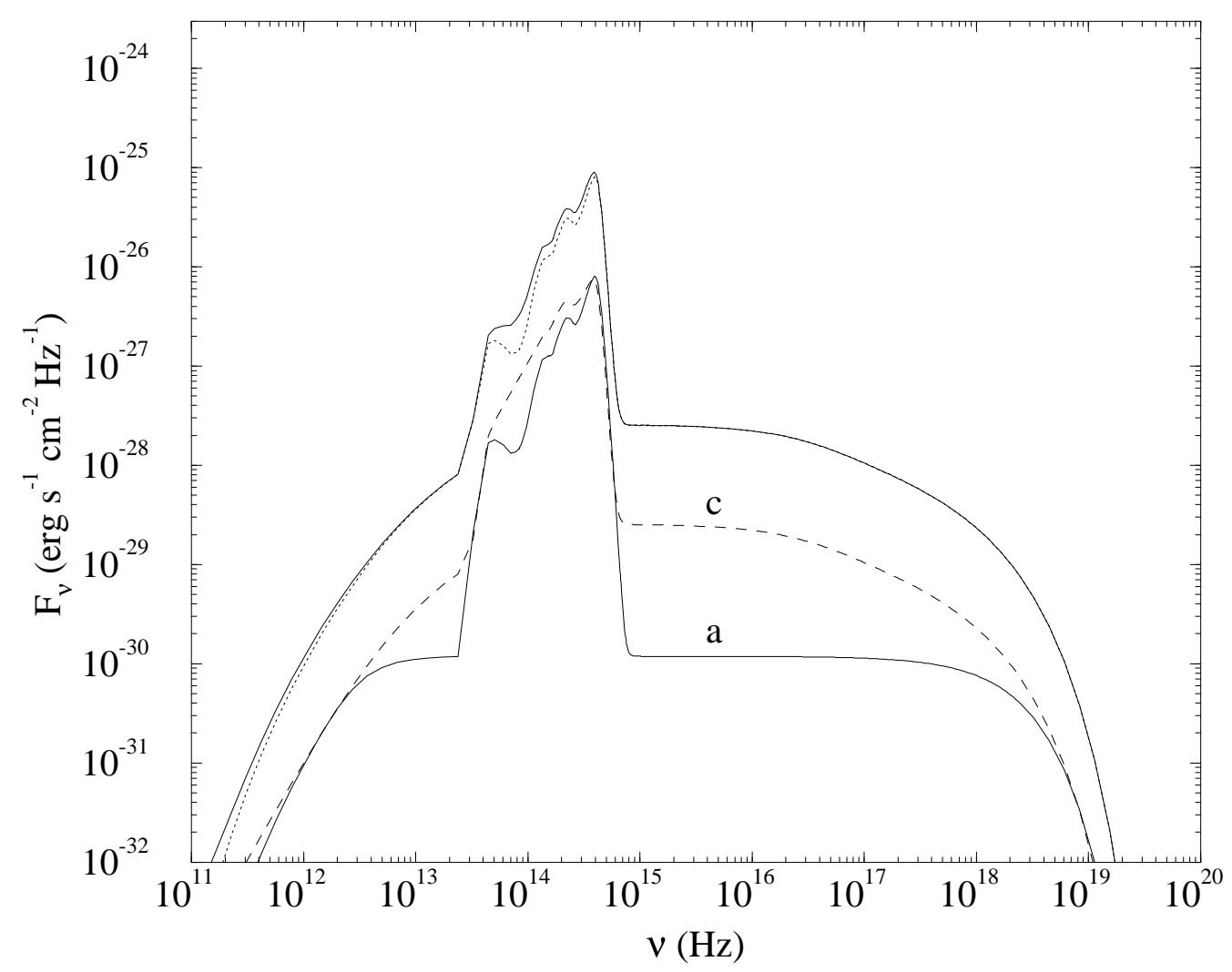

Abbildung 3.19: Vergleich des Spektrums aus einer als „unendlich ausgedehnt“ angenommenen Emissionsregion (gepunktete Linie), mit dem einer Säule $\left(D / h_{\mathrm{sh}}=\right.$ $1 / 2$; durchgezogene Linie), zu der Schichtung aus $M_{\mathrm{wd}}=0,6 M_{\odot}, \dot{m}=1 \mathrm{~g} \mathrm{~s}^{-1} \mathrm{~cm}^{-2}$ und $B=30 \mathrm{MG}$, unter dem Sichtwinkel $\vartheta=10^{\circ}$. Weiterhin sind die Flüsse zweier Strahlen aus der Unterteilung der Säule dargestellt: (a) Strahl beginnt an Seitenfläche und endet an der Oberfläche der Säule, (c) Strahl von Grundfläche aus endet an Säulenseite (Abb. 3.18).

gelten. Insbesondere fallen Temperatur und Dichte zu den Seiten hin nicht ab, d. h. die hydrodynamische Struktur soll von den begrenzenden Rändern nicht beeinflußt werden. Mit dieser Annahme der ausschließlichen Höhenabhängigkeit der Temperaturstruktur (statt eines realistischeren Temperaturabfalls zu den Rändern hin), sind die berechneten Spektren jedoch für qualitative Vergleiche sinnvoll. Statt die Intensität mit der Lösung der Strahlungstransportgleichung durch die gesamte Schicht zu bestimmen, wird nun die Region in eine Anzahl von „Strahlen“ eingeteilt, die das Emissionsvolumen vollständig überdecken (Abb. 3.18, vorherige Seite). Je nach Sichtwinkel $\vartheta$ zur Normalen ergeben sich einzelne Strahlen, die von der Grundfläche beginnend nicht am Anfang der Schicht enden (Strahl b in Abb. 3.18), sondern an einer Seitenfläche austreten (Strahl c), bzw. dort beginnen und am oberen Rand die Emissionsregion verlassen (Strahl a). Entlang jedes Strahls wird nun die Strahlungstransportgleichung gelöst und die resultierenden Intensitäten aufintegriert, um den Gesamtfluß der Emissionsregion zu erzeugen. Für die optisch dünnen Bereiche der Region ergeben sich keine Unterschiede zwischen den beiden Betrachtungsweisen, da 


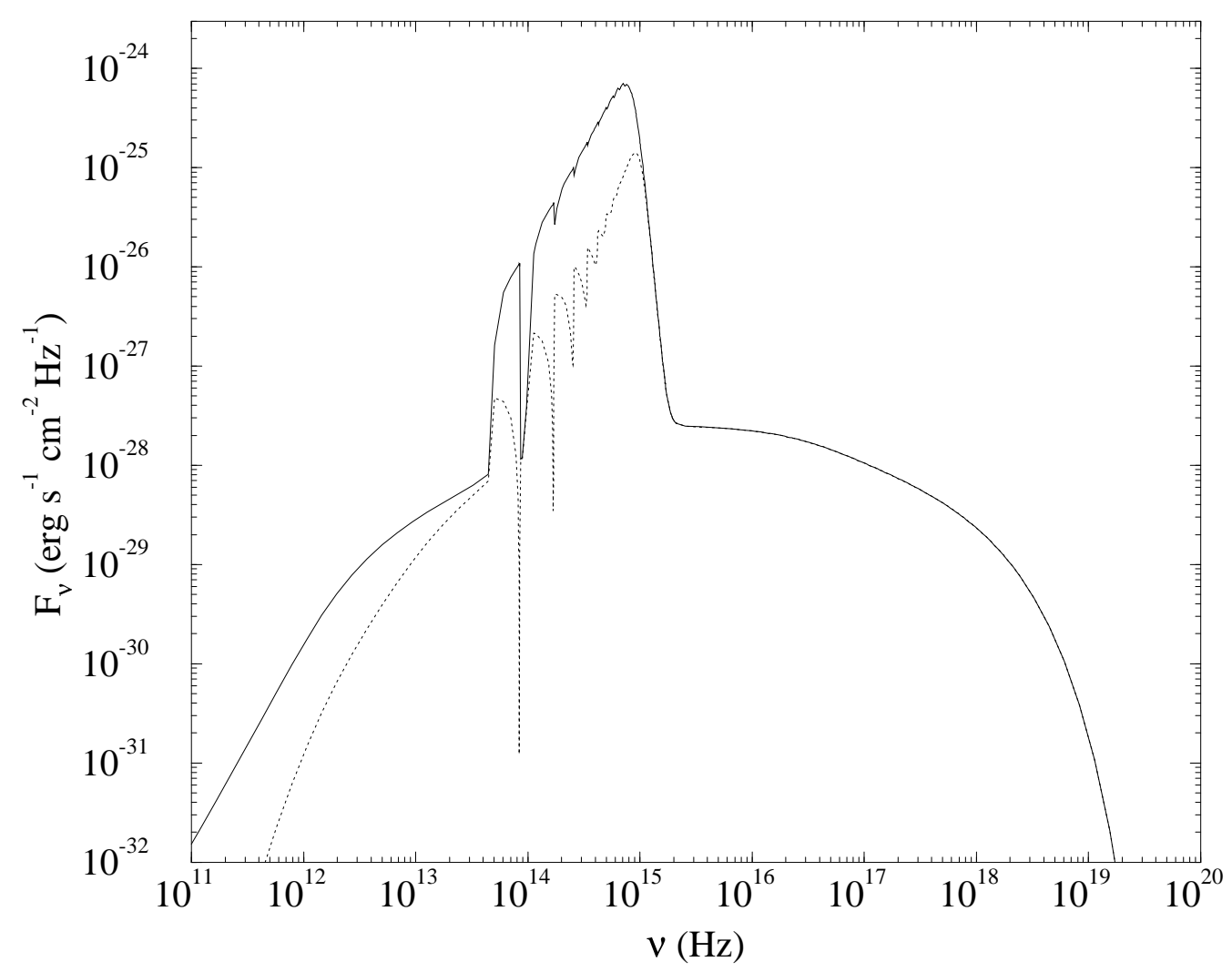

Abbildung 3.20: Darstellung der Spektren aus einer „unendlich ausgedehnten“ Emissionsregion und einer schmalen Säule wie in Abbildung 3.19, aber zu $\vartheta=80^{\circ}$.

bei der Integration der Emissivitäten nur das Volumen der Schicht eingeht, nicht aber ihre Geometrie. Ein Strahl, der nach wie vor die gesamte Schicht durchsetzt (Strahl b), erreicht die gleiche optische Tiefe wie im unendlich ausgedehnten Fall; die Spektren werden sich qualitativ also nicht unterscheiden. Der absolute Fluß ist jetzt allerdings geringer als im unendlich ausgedehnten Fall, da er nur Teil des Gesamtflusses der zerlegten Region ist. Dagegen erreicht ein Strahl, der die Emissionsregion durch die Seitenfläche verläßt, große optische Tiefen in einer Höhe, die unterhalb des Austrittsorts liegt. Der zugehörige Schwarzkörperfluß entstammt damit aus Regionen, deren Temperatur im unendlich ausgedehnten Fall nicht zu sehen wäre, da die optische Tiefe (wegen der längeren Wegstrecke) schon früher erreicht worden wäre (gestrichelte Verlängerung von Strahl c). Die Strahlen, die an einer Seite beginnen (Strahl a), werden wegen der geringen Temperaturen und Dichten am Anfang der Schicht nur sehr wenig zum Gesamtfluß beitragen.

In Abbildung 3.19 sind die Spektren der Emissionsregion zu einer Schichtung mit $M_{\mathrm{wd}}=0,6 M_{\odot}, \dot{m}=1 \mathrm{~g} \mathrm{~s}^{-1} \mathrm{~cm}^{-2}$ und $B=30 \mathrm{MG}$ unter dem Sichtwinkel $\vartheta=10^{\circ}$ dargestellt. Verglichen wird die Emission einer Säule des Verhältnisses $D / h_{\mathrm{sh}}=1 / 2$ mit dem Fluß aus einer als „unendlich ausgedehnt" angenommenen Akkretionsregion, die auf das gleiche Volumen reduziert ist. Das Spektrum der Säule wird durch eine Anzahl von Teilstrahlen gebildet, von denen zwei exemplarisch in der Darstellung angegeben sind. Die 
Kurve zu (a) entspricht dem in Abbildung 3.18 mit dem gleichen Buchstaben bezeichneten Fall, daß der Strahl von einer Seite der Säule ausgeht und den oberen Rand der Emissionsregion erreicht. Dessen Struktur des Flusses im optisch dicken Zyklotronanteil entspricht der Absorption in den kühlen Gebieten am Anfang der geheizten Schicht und ist mit dem Ergebnis aus der unendlich ausgedehnten Region identisch; lediglich der Absolutwert ist geringer, da es sich hier um einen Anteil am Gesamtfluß handelt. Die geringe Bremsstrahlung resultiert aus den kleinen Dichten am Anfang der Schicht. Der Fluß des Teilspektrums (c) wird durch einen Strahl gebildet, der am Boden der Emissionsregion entspringt und aus einer Seitenfläche austritt (Abb. 3.18). Wegen der Annahme, daß die Schichtung der Temperatur und Dichte über die gesamte Breite unverändert bleibt, entspricht dem optisch dicken Zyklotronstrahlungsfluß ein isothermer Schwarzkörper zur Temperatur der Höhe in der $\tau_{\nu} \gg 1$ wird; eine Absorptionsstruktur durch eine zum Rand hin abnehmende Temperatur tritt damit nicht auf. Der Bremsstrahlungsfluß ist größer als im Teilstrahl (a), da hier die Gebiete hoher Dichte und niedriger Temperatur am Ende der geheizten Schicht durchquert werden. Der in der Abbildung 3.19 aus Gründen der Übersichtlichkeit nicht dargestellte Fall (b) ergibt sich durch die Kombination von Teilen der Spektren aus (a) und (c): Wegen der Absorption in den hohen Gebieten der Schicht, weist dieser Teilstrahl die Absorptionsstruktur von (a) auf, und da er am Boden der Emissionsregion entspringt, ist die optisch dünne Emission identisch mit der von (c). Aus der Überlagerung dieser Eigenschaften entsteht ein Spektrum, das dem aus der unendlich ausgedehnten Schicht (bis auf den Absolutfluß) entspricht. Der Gesamtfluß der Säule wird nun durch die Aufintegration der Beiträge der Teilstrahlen gebildet. Da bei dem kleinen Sichtwinkel von $\vartheta=10^{\circ}$ die Emission der Seitenflächen nur wenig eingeht, überwiegen die Strahlen, die die gesamte geheizte Schicht durchqueren, und die Abweichungen zu dem Spektrum aus der unendlich ausgedehnten Emissionsregion sind nur gering.

Bei festem Verhältnis $D / h_{\text {sh }}$ wird der Einfluß der Seiteneffekte auf das Säulenspektrum mit zunehmendem Sichtwinkel gegen die Normale (Feldrichtung) stärker werden, so daß sich die Unterschiede zu einem Spektrum aus der unendlich ausgedehnten Schicht vergrößern. In Abbildung 3.20 (vorherige Seite) werden die Ergebnisse aus den beiden Modellen miteinander verglichen, wobei die selben Bedingungen wie in Abbildung 3.19 gelten, nur der Sichtwinkel beträgt jetzt $\vartheta=80^{\circ}$. Der optisch dünne Anteil des Spektrums ist, wie erwartet, in beiden Beschreibungen identisch. Dagegen zeigen sich im optisch dicken Teil deutliche Unterschiede. Während im Fall der unendlich ausgedehnten Emissionsregion starke Absorptionsstrukturen dominieren, gleicht das Säulenspektrum eher einem durch einen Schwarzkörper begrenzten Fluß. Wegen des hohen Sichtwinkels durchqueren nur sehr wenige Strahlen die gesamte geheizte Schicht, so daß deren Absorptionsstrukuren kaum zum Gesamtfluß beitragen. Die meisten Teilstrahlen werden durch die Seitenfläche austreten und damit fast isotherme Schichten passieren, da angenommen wurde, daß Temperatur und Dichte in zur Grundfläche parallelen Schnitten konstant ist. Ein Teilspektrum wird im optisch dicken Bereich daher in etwa ein Schwarzkörper zur Temperatur des parallelen Schnitts sein. Aus der Überlagerung der Mehrzahl dieser schwarzkörperartigen Emissionen bildet sich das Gesamtspektrum der Säule. 
Die Unterschiede zwischen den Spektren einer unendlich ausgedehnten und einer begrenzten Region werden sich auch auf die abgestrahlte Energie der Schicht auswirken und damit auf die Kühlung der Emissionsregion. Bestimmt man an Hand der Strahlung aus der unendlich ausgedehnten Schicht deren Energieinhalt, findet man die durch die Akkretion eingebrachte Energie wieder. Die Bedingung für eine stationäre Lösung der hydrodynamischen Gleichungen ist erfüllt, und die verwendete Temperaturstruktur ist konsistent mit der Strahlungskühlung. Berechnet man hingegen die von einer Säule zu dieser Temperaturund Dichteverteilung abgestrahlte Energie, so liegt diese über dem bei der Akkretion freigesetzten Wert; Energieerhaltung ist nicht mehr gewährleistet. Der Grund dafür ist, daß die optische Tiefe $\tau_{\nu} \gg 1$ in den beiden Modellen in unterschiedlichen geometrischen Höhen der Schicht erreicht wird. Zum Beispiel weist die Zyklotronstrahlung in den Maxima der niedrigen Harmonischen schon nach geringen Weglängen hohe optische Tiefen auf. Im Modell der unendlich ausgedehnten Schicht also in Gebieten des Temperaturanstiegs am Anfang, mit entsprechend geringem Schwarzkörperfluß und resultierender Absorptionsstruktur unter allen Sichtwinkeln zur Akkretionsfläche. Die bei diesen Frequenzen einbehaltene Energie trägt zur Heizung der Schicht bei und wurde bei der Berechnung der Temperaturstruktur mit berücksichtigt. In der Säule werden hohe optischen Tiefen, für Strahlen die die Seite der Emissionsregion verlassen, bei höheren Temperaturen am Ende der Schicht erreicht und ergeben einen entsprechend größeren Schwarzkörperfluß ohne Absorptionsstrukturen. Diese verstärkte Energieabstrahlung im Zyklotronanteil des Spektrums wurde allerdings bei der Berechnung der Temperaturstruktur nicht berücksichtigt. Die mit den Stahlungsverlusten der unendlich ausgedehnten Schicht berechnete Struktur der Emissionsregion muß im Fall der stärkeren Abstrahlung aus der Säule also einen höheren Energieinhalt liefern. Die Differenzen werden mit wachsendem Anteil der Seiteneffekte, d. h. bei einer Abnahme des Verhältnisses $D / h_{\text {sh }}$ und Zunahme des Zyklotronanteils am Gesamtspektrum, größer. Resultate aus Rechnungen über einen Bereich der Parameter Masse $M_{\text {wd }}$, Massenstromdichte $\dot{m}$ und Magnetfeldstärke $B$, sowie Seitenverhältnis $D / h_{\mathrm{sh}}$, sind in Abbildung 3.21 (folgende Seite) dargestellt. Darin ist das Verhältnis der Leuchtkraft einer begrenzten Säule $\left(L_{\mathrm{rad}}\right)$ zu der aus der unendlich ausgedehnten Schicht $\left(L_{\mathrm{rad}}(\infty)\right)$ für eine bestimmte Parameterkombination, in Abhängigkeit des Seitenverhältnisses der Säule angegeben. Der erwartete Verlauf des Energieverhältnisses mit dem Seitenverhältnis ist deutlich zu sehen: Unabhängig von einer speziellen Wahl der Parameter wird mit zunehmendem Seitenverhältnis auch das Energieverhältnis gegen Eins gehen, da die Einflüsse der Seiten immer geringer werden und eine Schicht, die breiter als hoch ist, der unendlich ausgedehnten Emissionsregion immer ähnlicher wird. Weiterhin nimmt das Energieverhältnis - bei konstanter Akkretionsrate und Geometrie - mit zunehmender Magnetfeldstärke zu, da das Spektrum der Emissionsregion nun wesentlich durch die Zyklotronstrahlung gebildet wird, und die Abweichungen der Säulenemission zur Strahlung der unendlich ausgedehnten Schicht hier besonders hoch sind. Die Erhöhung der Masse des weißen Zwergs hat den gleichen Effekt: Bei Konstanz der anderen Parameter ist das Energieverhältnis bei der größeren Masse höher. Die höhere Masse hat eine größere Stoßtemperatur zur Folge, und damit ebenfalls höhere Temperaturen in der Schicht, so daß die Seitenstrahlen der Säule durch einen Schwarzkörper zu einer höheren Temperatur 


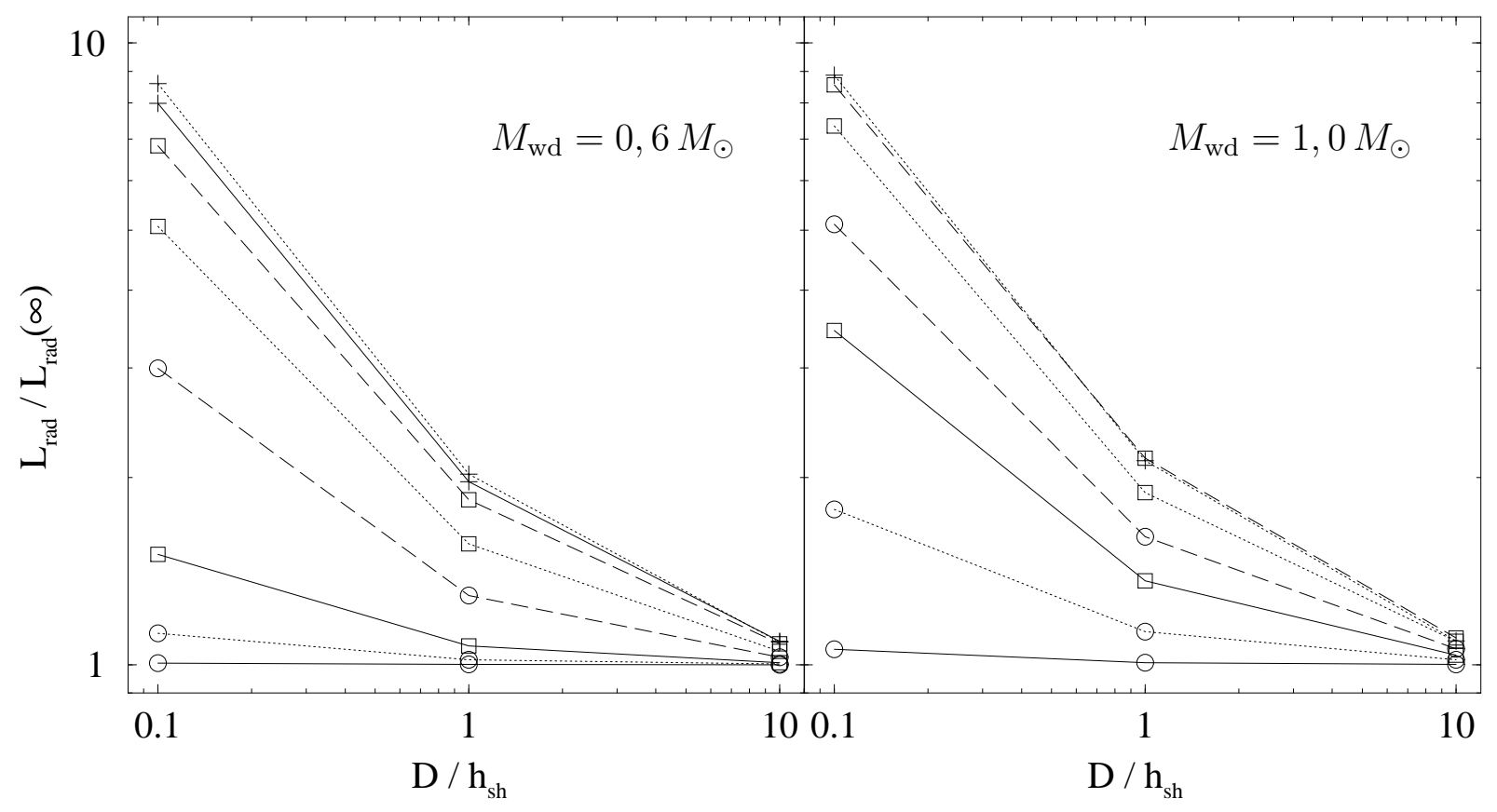

Abbildung 3.21: Verhältnis der Leuchtkraft einer Säule $\left(L_{\mathrm{rad}}\right)$ des Seitenverhältnisses $D / h_{\mathrm{sh}}$, zu der Leuchtkraft der unendlich ausgedehnten Emissionsregion $\left(L_{\text {rad }}(\infty)\right)$, für eine bestimmte Schichtung aus dem Parameterraum. Die Kreuze stehen für den Parameter $\dot{m}=10^{-2} \mathrm{~g} \mathrm{~s}^{-1} \mathrm{~cm}^{-2}$, die Quadrate für $\dot{m}=1 \mathrm{~g} \mathrm{~s}^{-1} \mathrm{~cm}^{-2}$ und Kreise entsprechen $\dot{m}=100 \mathrm{~g} \mathrm{~s}^{-1} \mathrm{~cm}^{-2}$. Die Punkte mit Magnetfeldstärke $B=10 \mathrm{MG}$ werden durch die durchgezogene Linie verbunden, die gepunktete Linie entspricht $B=30 \mathrm{MG}$ und die gestrichelte steht für $B=100 \mathrm{MG}$.

erzeugt werden. Eine Vergrößerung der Akkretionsrate bewirkt dagegen, daß das Emissionsspektrum immer stärker durch die optisch dünne Bremsstrahlung gebildet wird und die Unterschiede zwischen den Modellen kleiner werden. An Hand der Abbildung 3.21 kann nun leicht abgeschätzt werden, wie stark der Energieüberschuß der Säule bei einem vorgegebenen Parametersatz ist.

Die beschriebenen Effekte für Spektrum und Energieinhalt von schmalen Säulen sind auf die Annahme der Gültigkeit der hydrodynamischen Schichtung über die gesamte Breite der Emissionsregion zurückzuführen. Eine Absorptionsstruktur in den Spektren - auch unter hohen Sichtwinkeln - könnte z. B. durch einen ad hoc anzunehmenden Temperaturabfall und Dichtegradienten zu den Rändern hin erzeugt werden und würde somit die Abweichungen der Säulenspektren bzw. das Energieverhältnis $L_{\mathrm{rad}} / L_{\mathrm{rad}}(\infty)$ mildern. Diese zu erwartende Struktur ist aber nur aus vollständig zwei- bzw. dreidimensionalen Berechnungen der Hydrodynamik und des Strahlungstransports zu begründen, die in Zukunft durchzuführen sind. Die Resultate dieses Kapitels können daher nur eine qualitative Bewertung der Auswirkungen geben, wenn bei der Berechnung von Spektren, statt der zur Herleitung der Schichtungen benutzten unendlich ausgedehnten Emissionsregion, eine geometrische Struktur wie die einer begrenzten Säule benutzt wird. 


\section{Kapitel 4}

\section{Anwendung der Ergebnisse auf den Polar AM Herculis}

In diesem Kapitel werden die Ergebnisse der oben beschriebenen theoretischen Rechnungen auf ein konkretes System, den Polar AM Herculis (im folgenden AM Her abgekürzt), angewendet. Dafür wird die unter Modellannahmen über die stationäre Emissionsregion auf dem akkretierenden weißen Zwerg berechnete Strahlung an die aus verschiedenen Bereichen des elektromagnetischen Spektrums vorliegenden Beobachtungen angepaßt. Aus dem Vergleich von Theorie und Beobachtung können Aussagen über die Verhältnisse in der Akkretionsregion des Sterns getroffen werden, die ein weiteres Verständnis der zugrunde liegenden Prozesse der Akkretion ermöglichen. Die gefundenen Resultate stellen Teilaspekte des allgemeineren Problems der instationären Akkretion dar und werden im Diskussionsteil 4.2.3 in diesen Kontext eingeordnet.

\subsection{Modellierung der optischen Lichtkurve}

Eine charakteristische Eigenschaft der magnetischen CVs ist ihre zeitveränderliche Helligkeit mit Perioden zwischen 1,3 und 8,0 h, die in Form einer Lichtkurve in verschiedenen Frequenzbereichen des elektromagnetischen Spektrums registriert wird. Diese Intensitätsschwankungen werden im Modell der magnetisch beeinflußten Akkretion durch die unterschiedlichen Sichtwinkel zur Emissionsregion, die sich bei einer Drehung des Doppelsternsystems um seine Achse ergeben, erklärt. Dabei ist die Intensität der Röntgenstrahlung am höchsten, wenn der Akkretionsfleck vollständig sichtbar ist und geht gegen Null bei zunehmendem Sichtwinkel zur Flächennormalen. Der variable Anteil der Emission im optischen bis infraroten Spektralbereich wird dagegen durch die Zyklotronstrahlung dominiert, deren Richtungscharakteristik die Phasen maximaler bzw. minimaler Intensität bestimmt: Zyklotronstrahlung wird vornehmlich senkrecht zum Magnetfeld abgestrahlt. (Abb. 3.7, S. 57).

In einer Anzahl von Polaren wird neben einem ausgeprägtem optischen Minimum zu einer bestimmten Phase noch eine weitere Intensitätsabnahme (sekundäres Minimum) im Zentrum des Helligkeitsmaximums beobachtet (z. B. AM Her: Szkody \& Brownlee 1977; 
V834 Cen: Cropper, Menzies \& Tapia 1986). Ein Erklärungsmodell, das auch die Analyse der Polarisation der Strahlung einbezieht, geht von einer Magnetfeldgeometrie aus, in der die Akkretion an zwei Polen geschieht (Wickramasinghe et al. 1991). Durch die Überlagerung der Strahlung beider Pole können - bei geeigneter Wahl der Lage und Ausdehnung der Akkretionsregionen - sowohl das primäre als auch das sekundäre Minimum erzeugt und die Form der beobachteten Lichtkurve hinreichend genau reproduziert werden. Die hohe Zahl der freien Parameter des Modells beeinträchtigt allerdings die Aussagekraft der Ergebnisse. In diesem Kapitel soll gezeigt werden, daß die beschriebene Form der Lichtkurve auch mit einer einzelnen Emissionsregion erklärt werden kann. In diesem Modell ist die Anzahl der freien Parameter wesentlich geringer und kann somit zu einer einfacheren Klärung der Akkretionsvorgänge beitragen, auch wenn dieses Modell vielleicht nicht für alle Objekte dieser Klasse von ausreichender Tragfähigkeit ist.

In den obigen Kapiteln wurde beschrieben, daß bei einer hohen Akkretionsrate (engl. "high-state") die Energie in Form der Stoßheizung und nachfolgender Kühlung eines Plasmas freigesetzt wird. Die Höhe des Stoßes über der Sternoberfläche hängt dabei von der Effektivität der Kühlung des Plasmas ab. Die Emissionsregion erhebt sich damit um einen u. U. beträchtlichen Prozentsatz des Radius über den Stern und kann in den oberen Teilen weiterhin sichtbar sein, während die Basis bei der Orbitalbewegung des Systems schon durch den weißen Zwerg bedeckt wird. Bei diesem Vorgang überschreitet der Sichtwinkel $\vartheta$ zu einem senkrecht auf der Sternoberfläche stehendem Magnetfeld im laufe des Orbits auch den Wert $90^{\circ}$. Die Intensität der Zyklotronstrahlung wird also von einem Maximalwert wieder abnehmen, denn Winkel $\vartheta_{1}>90^{\circ}$ erzeugen eine Strahlung die äquivalent zu solcher für $\vartheta_{2}=180^{\circ}-\vartheta_{1}<90^{\circ}$ ist. Da das Magnetfeld im allgemeinen Fall nicht senkrecht auf der Oberfläche steht, kann dieser Durchgang durch $90^{\circ}$ für bestimmte Blickrichtungen noch besser sichtbar sein. Am Beispiel des Polars AM Her soll nun gezeigt werden, daß die optische Intensitätsvariation durch das Modell einer radial ausgedehnten Akkretionssäule mit einfachsten Annahmen in wesentlichen Punkten dargestellt werden kann.

Zur Berechnung einer Lichtkurve sind Annahmen über die Geometrie des Doppelsternsystems nötig. Aus den spektralen und photometrischen Variationen des Sekundärsterns über die Orbitalperiode hinweg läßt sich die Inklination (d.h. der Winkel zwischen Drehachse des Sterns und Sichtlinie) $i \simeq 50^{\circ}$ bestimmen (Southwell et al. 1995). Unter Verwendung dieses Wertes zeigen Gänsicke et al. (1998), daß sich die beobachtete UV-Lichtkurve mit einer geheizten Region bei einem Polabstand $\beta=55^{\circ}$ erklären läßt. Damit sind die bestimmenden Elemente der Systemgeometrie festgelegt. Wenn das Magnetfeld in der Akkretionsregion der Einfachheit halber als senkrecht auf der Oberfläche stehend angenommen wird, dann ergibt sich bei der Drehung des Polars der in Abbildung 4.1 dargestellte Verlauf des Sichtwinkels $\vartheta$ zum Magnetfeld. Die Phasenkonvention bezieht sich für $\phi_{\text {mag }}=0$ auf das Zentrum des beobachteten Intensitätsmaximums der linearen Polarisation (d.h. den Zeitpunkt maximaler Zyklotronstrahlung), während die Orbitalphase $\phi_{\text {orb }}=0$ mit dem Maximum der Röntgenstrahlung verknüpft ist $\left(\phi_{\text {orb }}=\phi_{\text {mag }}+0,367\right)$. Bei $\phi_{\text {mag }} \approx-0,1$ ist der Sichtwinkel $\vartheta \approx 90^{\circ}$. Dreht sich der Stern weiter, so vergrößert sich der Winkel bis auf $105^{\circ}$, einem für die Zyklotronstrahlung äquivalenten Wert von $\vartheta=75^{\circ}$. In dieser Position ist die Basis der Akkretionssäule durch den Horizont des wei- 


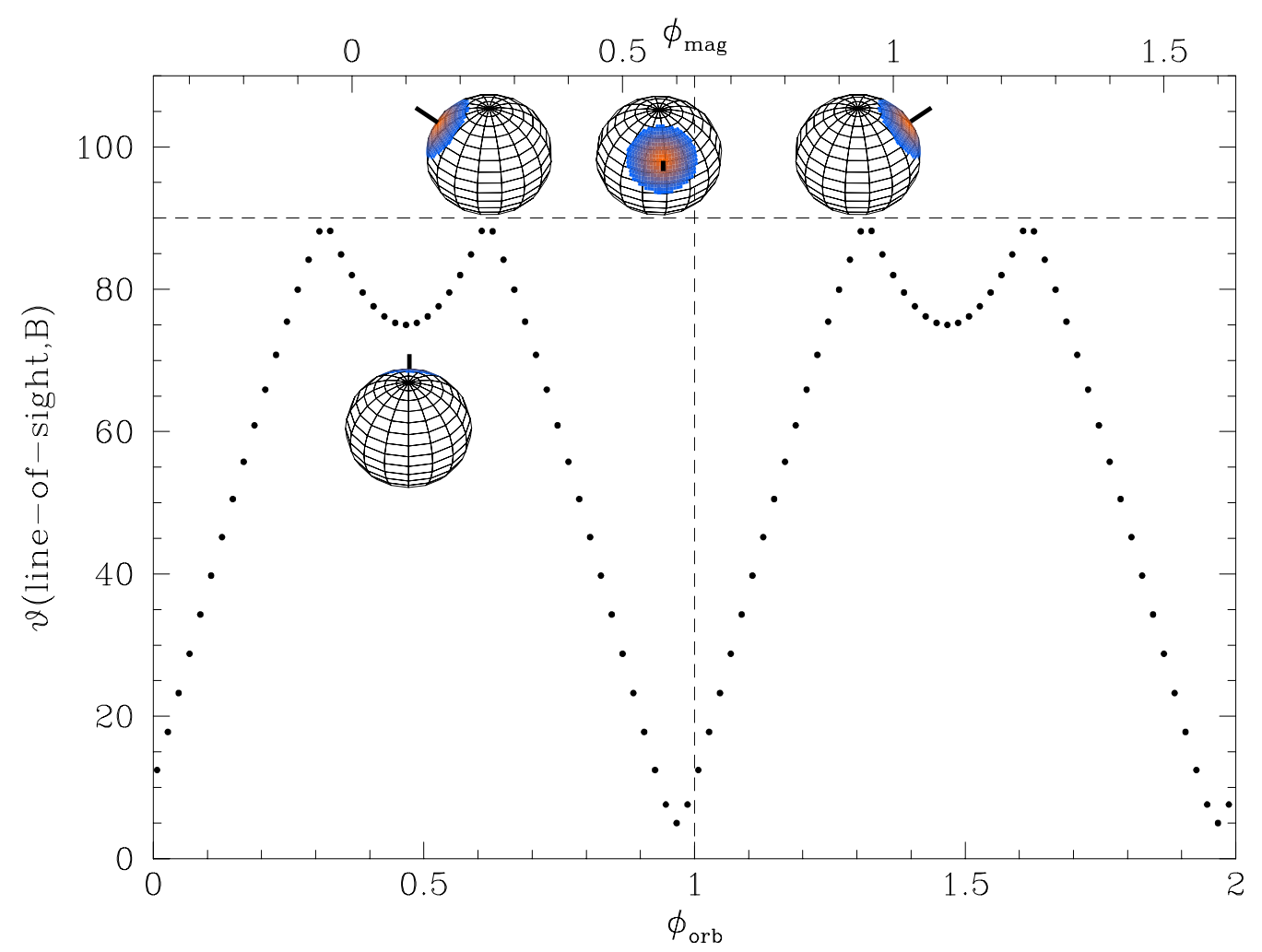

Abbildung 4.1: Sichtwinkel $\vartheta$ zum Magnetfeld als Funktion der Phase $\phi$. Eingezeichnet ist weiterhin die schematische Ansicht des weißen Zwergs mit durch die Strahlung der Akkretionssäule geheizter Oberfläche und eine „Akkretionssäule“ der Höhe $h \approx 10 \% R_{\mathrm{wd}}$. (Ich danke Dr. B.T. Gänsicke für die Erstellung dieser Abbildung.)

ßen Zwergs bedeckt, und nur die höheren Teile sind unter diesem Winkel sichtbar. Die zugehörige Intensität der Zyklotronstrahlung ist nun geringer als bei Phase $\phi_{\text {mag }} \approx-0,1$. Schreitet die Bahnbewegung weiter fort, erhöht sich der Sichtwinkel, bis er bei $\phi_{\text {mag }} \approx 0,3$ erneut $\vartheta \approx 90^{\circ}$ erreicht. Diesem Maximum folgt das absolute Minimum der Sichtwinkelverteilung, einer minimalen Zyklotronstrahlung entsprechend $\left(\phi_{\text {mag }} \approx 0,6\right.$ mit $\left.\vartheta \approx 5^{\circ}\right)$. Die schematische Darstellung des weißen Zwergs in Abbildung 4.1 mit angedeuteter Akkretionssäule und geheiztem Fleck verdeutlicht den beschriebenen Winkelverlauf und die Ansichten des Systems bei den verschiedenen Phasen.

Für einen leuchtkräftigen Zustand des CVs AMHer läßt sich aus der beobachteten Strahlung im infraroten, optischen und harten Röntgenbereich des Spektrums eine Akkretionsrate von $\dot{M}=5 \cdot 10^{15} \ldots 10^{16} \mathrm{~g} \mathrm{~s}^{-1}$ herleiten (Gänsicke, Beuermann \& de Martino 1995); dabei wurde die Masse zu $M_{\text {wd }}=0,6 M_{\odot}$ und die Entfernung zu $d=90$ pc angenommen. Die Polfeldstärke des weißen Zwergs ist in guter Näherung $B=14 \mathrm{MG}$ (Bailey, Ferrario \& Wickramasinghe 1991), so daß sich für die angegebene Akkretionsrate in einer dipolaren Magnetfeldgeometrie eine Akkretionsfläche von $A_{\text {acc }} \approx 5 \cdot 10^{16} \mathrm{~cm}^{-2}$ in der Nähe des Magnetpoles ergibt (Lubow \& Shu 1975, 1976; Heerlein, Horne \& Schwope 1999). Aus 
diesen Größen folgt eine mittlere Massenstromdichte von $\dot{m} \approx 0,02 \ldots 0,2 \mathrm{~g} \mathrm{~cm}^{-2} \mathrm{~s}^{-1}$. Zu diesen Parametern und dem aus Abbildung 1.1 resultierenden Sichtwinkelbereich wurde eine Anzahl von Zyklotronspektren berechnet und durch Faltung mit Filterkurven (Johnson 1965) für die Bänder V (,visuell“, Zentralwellenlänge $\left.\lambda_{0}^{\mathrm{V}}=0,55 \mu \mathrm{m}\right)$ und B („blau“, $\left.\lambda_{0}^{\mathrm{B}}=0,44 \mu \mathrm{m}\right)$ in Helligkeitswerte überführt. Dabei wurde jedoch nicht die Selbstbedeckung der unteren Teile der Akkretionssäule berücksichtigt, so daß eine Variation der Intensität allein auf der Winkelabhängigkeit der Zyklotronstrahlung beruht.

Als Beobachtungsergebnisse in den Bändern V und B liegen zeitlich hochaufgelöste photometrische Daten vor, die von Dr. R. Silvotti am 1,5 m-Teleskop in Loiano (NordItalien) im August 1998 aufgenommen wurden (Silvotti et al. 2000, in Vorbereitung). Mit dem Satz von berechneten Spektren wurde nun eine Anpassung] der beobachteten Lichtkurve vorgenommen, in die die oben beschriebene Geometrie $\left(i=50^{\circ}, \beta=55^{\circ}\right)$ eingeht. Darüberhinaus mußten noch Annahmen über die Beiträge des durch Bestrahlung aus der Akkretionssäule geheizten weißen Zwergs und des Akkretionsstroms getroffen werden, da diese Komponenten in den betrachteten Bändern z. T. wesentlich sind (Gänsicke 1999). Da außer der Massenstromdichte alle anderen Parameter durch unabhängige Betrachtungen festgelegt werden können, zielte die Anpassung auf eine Bestimmung von $\dot{m}$. Als Bestwert wurde $\dot{m}=0,06 \mathrm{~g} \mathrm{~cm}^{-2} \mathrm{~s}^{-1}$ gefunden; die resultierende Lichtkurve ist in Abbildung 4.2 dargestellt. Der Anteil der Zyklotronstrahlung entspricht dem in Abbildung 4.1 beschriebenen Verlauf der Sichtwinkelvariation: Die „Doppelmaxima“ werden durch Strahlung nahe $\vartheta=90^{\circ}$ erzeugt. Das Minimum bei $\phi_{\text {orb }} \approx 0,45$ resultiert aus Zyklotronstrahlung der erhabenen Akkretionssäule unter einem Winkel von $\vartheta \approx 75^{\circ}$. Als Stoßhöhe bei dem Bestwert $\dot{m}=0,06 \mathrm{~g} \mathrm{~cm}^{-2} \mathrm{~s}^{-1}$ findet man $h_{\mathrm{sh}} \approx 7 \cdot 10^{7} \mathrm{~cm}$. Dies entspricht einer Ausdehnung von $\approx 8 \% R_{\mathrm{wd}}$, so daß bei den benutzten Werten für Inklination und Lage des Akkretionsflecks zum Pol die Akkretionssäule tatsächlich beobachtet werden kann, obwohl deren Basis schon durch den Horizont des weißen Zwergs verdeckt wird. Das folgende Minimum $\left(\phi_{\text {orb }} \approx 1\right.$ ) entspricht einem geringen Zyklotronanteil unter dem Sichtwinkel $\vartheta \approx 5^{\circ}$; hier sind die Beiträge von Strom und weißem Zwerg dominant. Bei fortschreitender Bahnbewegung nimmt der Sichtwinkel wieder zu, und auch die Komponente aus der Zyklotronstrahlung verstärkt sich, um das zweite der Doppelmaxima zu erzeugen. Der Beitrag der Zyklotronstrahlung ist im V-Band zu allen Phasen stärker als im B-Band. Dies liegt in der charakteristischen Eigenschaft der optisch dünnen Zyklotronstrahlung begründet, deren Emission - bei fester Temperatur und Magnetfeldstärke - für hohe Frequenzen rasch abnimmt (Für die Zentralwellenlängen der Filterkurven gilt $\left.: \lambda_{0}^{\mathrm{B}}<\lambda_{0}^{\mathrm{V}}\right)$. Die Phasenvariation des Anteils vom weißen Zwerg wird durch die Sichtbarkeit des geheizten Flecks auf seiner Oberfläche bestimmt (Abb. 4.1): annähernd parallele Sicht zur Flächennormale $\left(\phi_{\text {orb }} \approx 1\right)$ erzeugt einen stärkeren Beitrag als ein Verschwinden des Flecks hinter dem Horizont $\left(\phi_{\text {orb }} \approx 0,5\right)$. Der Beitrag des Stroms ist im beobachteten Spektralbereich in erster Näherung konstant mit der Phase (Gänsicke, Beuermann \& de Martino 1995); eine phasenabhängige Variation würde ein detailliertes Modell dieser Komponente erfordern, das jedoch über diese Arbeit hinausgeht.

\footnotetext{
${ }^{1}$ Ich danke Dr. B.T. Gänsicke für die Durchführung der Anpassung an die Beobachtungsdaten.
} 


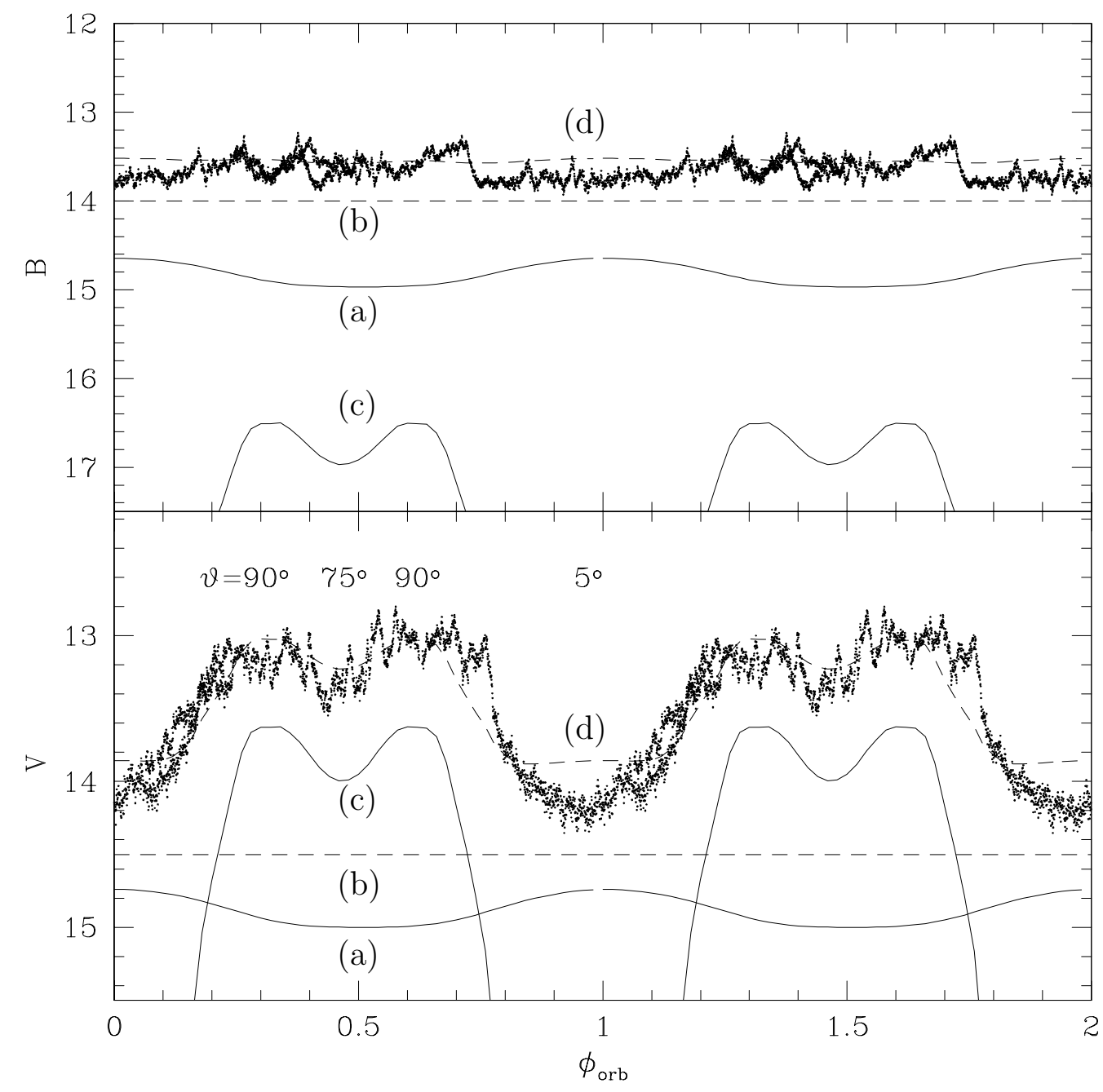

Abbildung 4.2: Lichkurve in den Filterbändern $\mathrm{V}\left(\lambda_{0}=0,55 \mu \mathrm{m}\right)$ und $\mathrm{B}$ $\left(\lambda_{0}=0,44 \mu \mathrm{m}\right)$ (Silvotti et al. 2000, in Vorbereitung). Dargestellt ist der Helligkeitsbeitrag (in mag) des geheizten weißen Zwergs (a), des Akkretionsstroms (b) und der Zyklotronstrahlung (c) als Funktion der Orbitalphase $\phi_{\text {orb }}$. Die resultierende Lichtkurve ist die gestrichelte Linie (d). (Ich danke Dr. B.T. Gänsicke für die Erstellung dieser Abbildung auf Grund der in dieser Arbeit berechneten Spektren.)

Mit den oben angegebenen einfachen Annahmen kann also die optische Lichtkurve von AM Her im „high-state" reproduziert werden, ohne den Einfluß einer zweiten Akkretionsregion zu berücksichtigen. Als einziger freier Parameter tritt dabei die Massenstromdichte auf, deren Variationsbreite zusätzlich noch durch die aus der Gesamtleuchtkraft resultierende Akkretionsrate $\dot{M}$ festgelegt ist. Die Defizite in der Anpassung bei $\left(\phi_{\text {orb }} \approx 1\right)$ könnten durch eine weitere Annahme über die Magnetfeldgeometrie, wie z. B. Neigung der Feldlinien im Fleck oder Variation der Feldstärke, behoben werden. Um dies zu rechtfertigen, ist aber eine genauere Abschätzung des Strombeitrags nötig, der im vorgestellten Modell zu dieser Phase dominant ist. Ein weiterer Hinweis auf Unsicherheiten in der Hel- 
ligkeit des Stroms ist die Tatsache, daß mit dem obigen Bestwert $\dot{m}=0,06 \mathrm{~g} \mathrm{~cm}^{-2} \mathrm{~s}^{-1}$ eine Verbesserung der V-Anpassung im Bereich $\phi_{\text {orb }}=0,8 \ldots 1,2$ durch eine Systemgeometrie mit $i=35^{\circ}$ und $\beta=70^{\circ}$ erreicht werden kann. Dazu ist es aber notwendig, den Beitrag des Stroms im V-Band um $0,5^{\mathrm{m}}$ zu verkleinern. Weiterhin sollten die Vorhersagen des Modells in anderen photometrischen Bändern (U, R, I, J, H, K) an Hand von entsprechenden Beobachtungen getestet werden, damit eine konsistente Beschreibung über den gesamten Spektralbereich der Zyklotronstrahlung gewährleistet ist.

\subsection{Spektrale Energieverteilung}

Polare zeigen Emission über einen weiten Bereich des elektromagnetischen Spektrums, die von der Radiostrahlung über den optischen Bereich bis zur harten Röntgenstrahlung und - z. B. in AMHer - in den Bereich der $\gamma$-Strahlung reicht. Nach dem allgemein akzeptierten Modell wird diese Strahlungsenergie durch Umwandlung von Gravitationsenergie bei der Massenakkretion auf einen weißen Zwerg gespeist. Je nach den Systemparametern Akkretionsrate $\dot{M}$ und Magnetfeldstärke $B$, ist das Maximum der Emission bei unterschiedlichen Frequenzen zu finden. Aus dieser beobachteten Verteilung der Energie im gesamten Spektralbereich eines konkreten Polars läßt sich nun wiederum auf die physikalischen Verhältnisse in dem System rückschließen.

\subsection{1 "High-state" Zustand des Systems}

Befindet sich ein magnetischer CV in einem leuchtkräftigen Zustand (engl. „high-state“), so ist das Modell der Energiefreisetzung durch einen hydrodynamischen Stoß über der Sternoberfläche gerechtfertigt. Dabei wird das akkretierte Material auf hohe Temperaturen geheizt und durch Strahlung gekühlt, wodurch sich eine Plasmasäule mit einer Temperatur- und Dichteschichtung zwischen Stoß und Oberfläche des weißen Zwergs ausbildet. Diese Säule heizt nun wiederum durch Strahlung die Sternoberfläche, so daß sich ein ausgedehnter Fleck hoher Temperatur in der Region des Auftreffpunkts des Akkretionsstroms bildet.

Am Beispiel des Polars AM Her konnte gezeigt werden, daß die UV-Strahlung $F_{\text {UV }}$ der geheizten Region eines weißen Zwergs mit der in der Sternoberfläche reprozessierten Zyklotron- $F_{\text {cyc }}$ und Bremsstrahlung $F_{\mathrm{ff}}$ der Akkretionssäule übereinstimmt (Gänsicke, Beuermann \& de Martino 1995). Die in diesem System - wie auch bei den Objekten UZ For, VV Pup, QQ Vul u. a. (Ramsay et al. 1994; Beuermann \& Burwitz 1995) - auftretende starke Komponente $F_{\mathrm{bb}}$ im weichen Röntgenbereich kann jedoch nicht durch Reprozessierung der beobachteten Flüsse in den anderen Spektralbereichen erklärt werden: Der Überschuß beträgt $\left(F_{\mathrm{bb}}+F_{\mathrm{UV}}\right) /\left(F_{\mathrm{cyc}}+F_{\mathrm{ff}}\right) \approx 7,3$ (Gänsicke, Beuermann \& de Martino 1995). Diese Diskrepanz wird in der engl. Literatur mit dem Begriff „Soft X-Ray Puzzle" bezeichnet. Ein Modell zur Klärung dieses Problems geht von der (vermutlich stark zeitvariablen) Freisetzung der Gravitationsenergie dichter „Klumpen“ des Akkretionsstroms unterhalb der Atmosphäre des weißen Zwergs aus, die sog. „blobby accretion“. 
Dabei wird die Energie in der Atmosphäre reprozessiert und als weiche Röntgenstrahlung re-emittiert (siehe Kap. 1.2.5). Durch eine zeitliche Änderung der Anzahl der akkretierten „Klumpen“ pro Zeit kann dieses Modell auch die beobachtete Variabilität der Röntgenstrahlung beschreiben.

In diesem Kapitel soll nun versucht werden, die spektrale Gesamtenergieverteilung des Polars AM Her - im Rahmen des Modells eines stationären hydrodynamischen Stoßes mit ausgebildeter Temperatur- und Dichteschichtung einer Akkretionssäule - wiederzugeben. Dabei wird sich ein Modell der weichen Röntgenkomponente ergeben, das die Energetik der auftretenden Strahlungsprozesse richtig wiedergibt.

Bei der für AM Her festgestellten Magnetfeldstärke $B \approx 14 \mathrm{MG}$ wird Zyklotronstrahlung im roten bis infraroten Bereich des elektromagnetischen Spektrums erwartet. Unter Verwendung ihrer während eines leuchtkräftigen Zustands des Polars im Juni 1977 durchgeführten zeitlich hochaufgelösten, photometrischen Beobachtungen in den Filterbändern $\mathrm{U}$ (Zentralwellenlänge $\left.\lambda_{0}=0,36 \mu \mathrm{m}\right), \mathrm{B}\left(\lambda_{0}=0,43 \mu \mathrm{m}\right), \mathrm{V}\left(\lambda_{0}=0,55 \mu \mathrm{m}\right)$, $\mathrm{R}\left(\lambda_{0}=0,7 \mu \mathrm{m}\right), \mathrm{I}\left(\lambda_{0}=0,9 \mu \mathrm{m}\right), \mathrm{H}\left(\lambda_{0}=1,65 \mu \mathrm{m}\right)$ und $\mathrm{K}\left(\lambda_{0}=2,2 \mu \mathrm{m}\right)$ leiten Priedhorsky et al. (1978) einen spektralen Verlauf für die Phasen $\phi_{\text {mag }} \approx 0,8$ und $\phi_{\text {mag }} \approx 0,6$ her, zu denen ein hoher bzw. niedriger Sichtwinkel zur lokalen Magnetfeldrichtung erwartet wird. Zur Elimination von Beiträgen des Akkretionsstroms, des weißen Zwergs und des Sekundärsterns wurde die Differenz der spektralen Energieverteilung der Phasen $\phi_{\text {mag }} \approx 0,8$ und $\phi_{\text {mag }} \approx 0,6$ gebildet. Die resultierenden Differenzen sind in Abbildung 4.3 (S. 87) dargestellt. Die Fehlergrenzen wurden aus den bei Priedhorsky et al. (1978) angegebenen Werten berechnet bzw. um systematische Werte durch Strom und weißen Zwerg in U und B, sowie durch den Sekundärstern in K erhöht.

Da zu den Positionen $\phi_{\text {mag }} \approx 0,8$ und $\phi_{\text {mag }} \approx 0,6$ Zyklotronstrahlung unter jeweils anderem Sichtwinkel zu erwarten ist, kann eine Anpassung durch Modellspektren nicht zu einem einzigen Winkel durchgeführt werden. Stattdessen muß ein Differenzspektrum aus den vermuteten Einzelwinkeln bei der jeweiligen Phase verwendet werden und in einer nachfolgenden Konsistenzprüfung mit dem beobachteten Verlauf des Spektrums zu der betreffenden Phase verglichen werden. Dafür wurden über einen Bereich von Massenstromdichten $\dot{m}=10^{-3} \ldots 100 \mathrm{~g} \mathrm{~cm}^{-2} \mathrm{~s}^{-1}$ und erwarteten Sichtwinkeln $\vartheta=90 \ldots 0^{\circ}$ Spektren der Akkretionssäule gerechnet, wobei die Masse des weißen Zwergs zu $M_{\mathrm{wd}}=0,6 M_{\odot}$ und das senkrecht auf der Sternoberfläche stehende Magnetfeld mit $B=14 \mathrm{MG}$ festgelegt wurden. Als Entfernung des Systems wurde $d=90$ pc gewählt. Der Vergleich der Differenzspektren mit dem beobachteten Verlauf ergab zunächst, daß für keine Kombination der Einzelwinkel - und auch zu keinem einzelnen Winkel - eine Anpassung mit nur einer Massenstromdichte gelingt, da die Beobachtung über einen breiten Wellenlängenbereich relativ hohen Fluß zeigt, während ein Modellspektrum zu einem festen $\dot{m}$ nur in einem engen Abschnitt des beobachteten Spektrums wesentlich beiträgt. Dieser Befund macht es daher erforderlich, eine Gewichtung der Beiträge aus einzelnen $\dot{m}$-Werten vorzunehmen, um somit das beobachtete Differenzspektrum zu erzeugen. Die Gewichtung erfolgt dabei als Veränderung der strahlenden Fläche der Säule. Wegen der Qualität der Beobachtung und der Unsicherheiten der Differenzbildung wurde eine $\dot{m}$-Diskretisierung in Potenzen von 10 angenommen: $\dot{m}=10^{-3} ; 10^{-2} ; 0,1 ; 1 ; 10 ; 100 \mathrm{~g} \mathrm{~cm}^{-2} \mathrm{~s}^{-1}$. Die untere Grenze ergibt 
sich aus der Unsicherheit der Beobachtung, da im Bereich des Beitrags der Massenstromdichten $\dot{m}<10^{-3} \mathrm{~g} \mathrm{~cm}^{-2} \mathrm{~s}^{-1}$ nur zwei Datenpunkte vorhanden sind, die eine weitere Diskretisierung unangemessen erscheinen lassen. Für $\dot{m}>100 \mathrm{~g} \mathrm{~cm}^{-2} \mathrm{~s}^{-1}$ liegt der Beitrag vor allem im Röntgenbereich und kann durch die infrarot-optischen Beobachtungen nicht aufgelöst werden.

Eine Anpassung mit verschiedenen Kombinationen der Sichtwinkel im synthetischen Differenzspektrum und unterschiedlichen Gewichtungen der einzelnen Massenstromdichten ergab zu der Phase $\phi_{\mathrm{mag}} \approx 0,8$ als Bestwerte für den Winkel zum Magnetfeld $\vartheta \approx 80^{\circ}$ und für die Phase $\phi_{\mathrm{mag}} \approx 0,6$ einen Wert $\vartheta \approx 5^{\circ}$. Die Flächengewichte $\Delta A_{\text {acc }}$ der einzelnen Massenstromdichten und deren differentieller Beitrag $\Delta \dot{M}$ zur Akkretionsrate $\dot{M}$ sind in folgender Tabelle angegeben:

\begin{tabular}{c|c|c}
$\dot{m}\left(\mathrm{~g} \mathrm{~cm}^{-2} \mathrm{~s}^{-1}\right)$ & $\Delta A_{\mathrm{acc}}\left(\mathrm{cm}^{2}\right)$ & $\Delta \dot{M}\left(\mathrm{~g} \mathrm{~s}^{-1}\right)$ \\
\hline 100 & $6,93 \cdot 10^{13}$ & $6,93 \cdot 10^{15}$ \\
10 & $2,00 \cdot 10^{14}$ & $2,00 \cdot 10^{15}$ \\
1 & $6,97 \cdot 10^{14}$ & $6,97(1,80) \cdot 10^{14}$ \\
0,1 & $2,04 \cdot 10^{15}$ & $2,04(0,31) \cdot 10^{14}$ \\
$10^{-2}$ & $1,56 \cdot 10^{16}$ & $1,56(0,70) \cdot 10^{14}$ \\
$10^{-3}$ & $1,32 \cdot 10^{16}$ & $1,32(1,32) \cdot 10^{13}$
\end{tabular}

Das gefundene synthetische Differenzspektrum ist zusammen mit den gewichteten Anteilen der einzelnen Massenstromdichten in Abbildung 4.3 dargestellt. Deutlich ist der Beitrag der einzelnen $\dot{m}$ zu bestimmten Wellenlängenbereichen erkennbar, der eine Gewichtung erforderlich machte. Für hohe Massenstromdichten liegt der Schwerpunkt der Emission bei kleinen Wellenlängen, einer Emission der hohen (optisch dünnen) Zyklotronharmonischen entsprechend. Für große Wellenlängen ist die Strahlung dieser Massenstromdichten - wegen der Selbstabsorption durch die Temperaturschichtung in der Säule durch den Verlauf der Schwarzkörperstrahlung begrenzt. Dagegen liegt für kleine Massenstromdichten der wesentliche Strahlungsanteil bei großen Wellenlängen des betrachteten Spektralbereichs. Da das beobachtete Spektrum dort nur durch zwei Punkte bestimmt ist, ist der Variationsbereich der Flächengewichte für die kleinen Massenstromdichten größer, und die angegebenen Werte weisen einen größeren Fehler auf als die mittleren $\dot{m}$. Allerdings bleibt der grobe Gang der Vergrößerung des Flächenanteils bei kleiner werdenden Massenstromdichten davon unberührt, da die Zyklotronstrahlung bei kleinen Maximaltemperaturen der Temperaturschichtung schwächer ist als bei großen Massenstromdichten.

Aus der Anpassung wird weiterhin deutlich, daß Massenstromdichten oberhalb $\dot{m} \approx$ $3 \mathrm{~g} \mathrm{~cm}^{-2} \mathrm{~s}^{-1}$ nur noch wenig zum Zyklotronspektrum beitragen können, da der beobachtete Intensitätsverlauf zu kleinen Wellenlängen hin schnell abfällt. Die Strahlung dieser sehr hohen Massenstromdichten kann daher nur aus kleinen Bereichen der Akkretionsregion stammen, da sonst der beobachtete Fluß überschritten würde. Allerdings tragen diese hohen $\dot{m}$-Werte entscheidend zur Akkretionsrate $\dot{M}$ bei: Mit obigen Werten ergibt sich ein - aus zusätzlichen Beobachtungen im harten Röntgenbereich motivierter - Wert für $\dot{M}=\Sigma \Delta \dot{M}=10^{16} \mathrm{~g} \mathrm{~s}^{-1}$, der zu $89 \%$ von den Massenstromdichten 


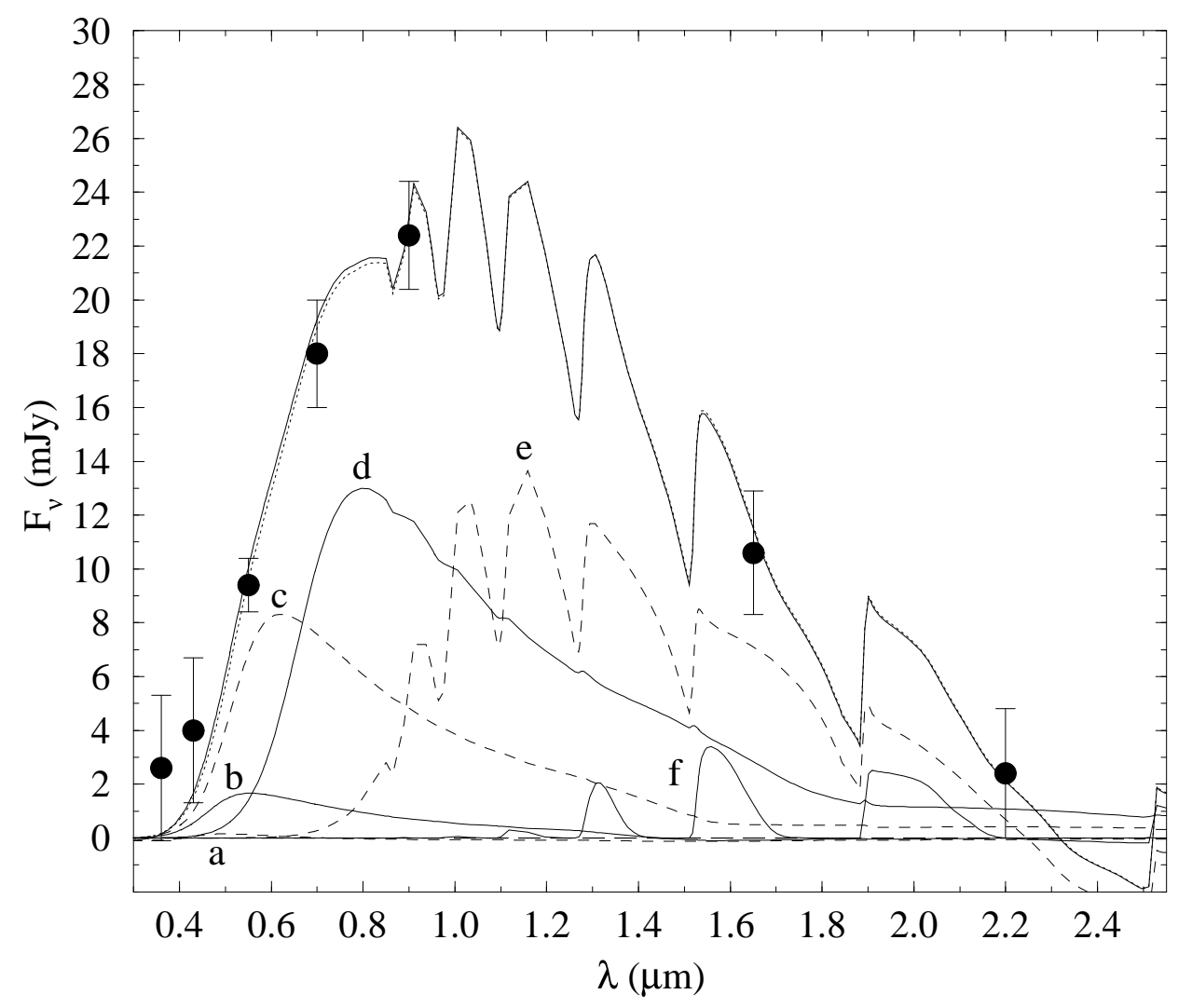

Abbildung 4.3: Anpassung des optischen bis infraroten Spektralbereichs eines „high-state“ Zustandes des Polars AMHer (1 mJy := $\left.10^{-26} \mathrm{erg} \mathrm{s}^{-1} \mathrm{~cm}^{-2} \mathrm{~Hz}^{-1}\right)$. Punkte: Differenzwerte aus Photometrie (UBVRIHK) zu den Phasen $\phi_{\text {mag }} \approx 0,8$ bzw. $\approx 0,6$ (Priedhorsky et al. 1978). Durchgezogene Linie: Differenz zwischen der Zyklotronstrahlung einer Akkretionssäule unter $\vartheta \approx 80^{\circ}$ und $\vartheta \approx 5^{\circ}$ für eine Akkretionsrate $\dot{M}=10^{16} \mathrm{~g} \mathrm{~s}^{-1}$. Die gewichteten Anteile der einzelnen Massenstromdichten darin sind: (a) $\dot{m}=100$, (b) 10 , (c) 1 , (d) 0,1 , (e) $10^{-2}$ und (f) $10^{-3} \mathrm{~g} \mathrm{~cm}^{-2} \mathrm{~s}^{-1}$. Gepunktete Linie: Anpassung zu $\dot{M}=5 \cdot 10^{15} \mathrm{~g} \mathrm{~s}^{-1}$.

$\dot{m}=10$ und $100 \mathrm{~g} \mathrm{~cm}^{-2} \mathrm{~s}^{-1}$ gestellt wird. Dagegen machen diese $\dot{m}$ nur $0,85 \%$ an der gesamten Akkretionsfläche von $A_{\text {acc }}=\Sigma \Delta A_{\text {acc }}=3,2 \cdot 10^{16} \mathrm{~cm}^{-2}$ aus. Zur Verdeutlichung dieses Umstandes ist in Abbildung 4.3 ebenfalls eine Anpassung gezeigt, deren Akkretionsrate nur die Hälfte des obigen Wertes ausmacht und die sich nur durch eine geringere Gewichtung der Massenstromdichten $\dot{m}=10$ und $100 \mathrm{~g} \mathrm{~cm}^{-2} \mathrm{~s}^{-1}$ von der Anpassung zu $\dot{M}=10^{16} \mathrm{~g} \mathrm{~s}^{-1}$ unterscheidet. Die Beiträge der $\dot{m}>3 \mathrm{~g} \mathrm{~cm}^{-2} \mathrm{~s}^{-1}$ sind so gering, daß an Hand der Beobachtung im Infraroten und Optischen allein kaum eine Aussage über die tatsächlich in dem CV herrschende Akkretionsrate getroffen werden kann, sondern das Röntgenspektrum mit in Betracht zu ziehen ist (s. unten). In obiger Tabelle sind daher keine Fehler im Beitrag dieser Massenstromdichten zur Akkretionsrate angegeben.

Da in der Anpassung Differenzen zwischen der Zyklotronstrahlung zu zwei Phasen gebildet werden, ist es notwendig, zu prüfen, ob die resultierenden Spektren zu jeder einzelnen Phase mit dem beobachteten Intensitätsverlauf konsistent sind. Daher sind in 


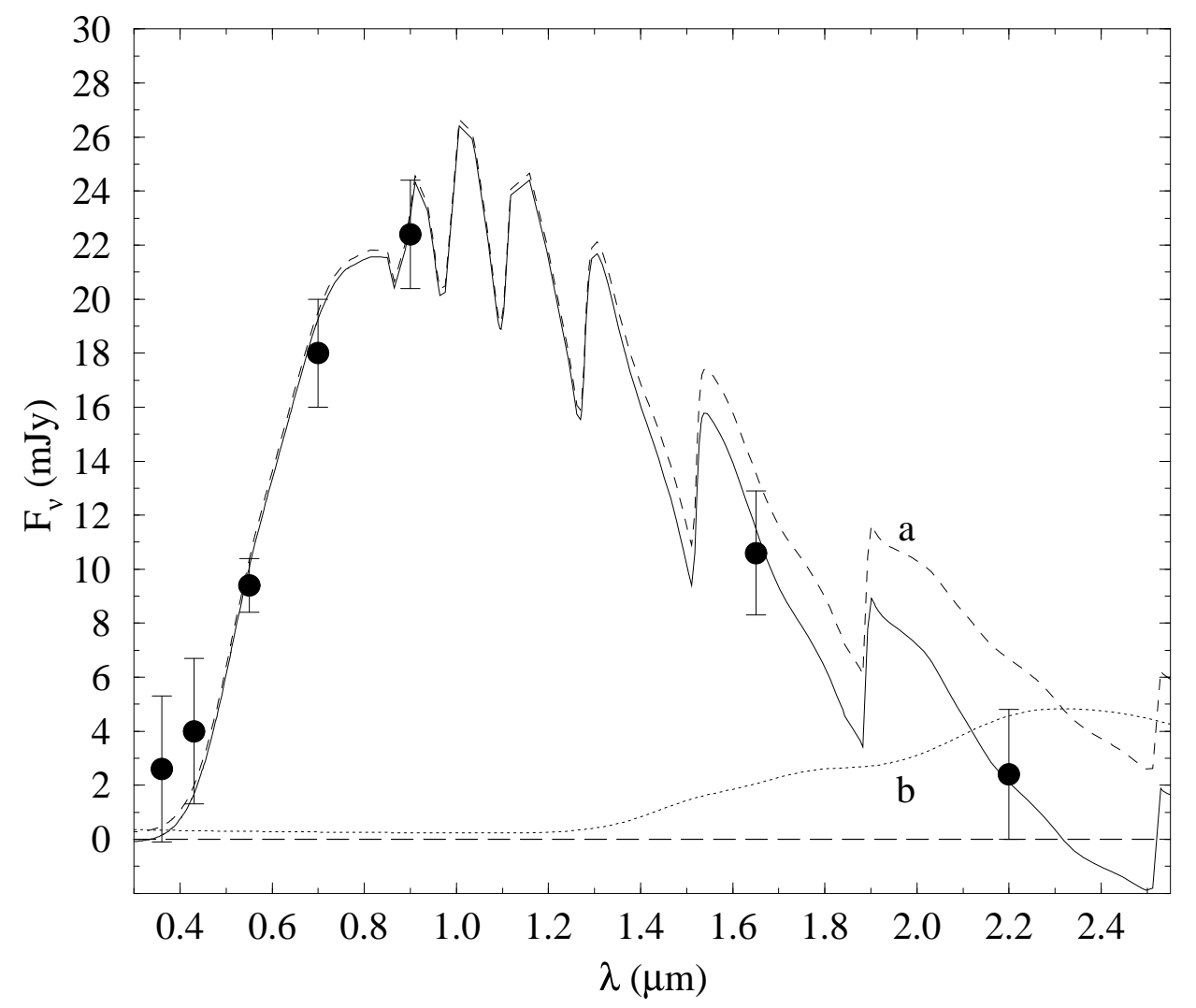

Abbildung 4.4: Winkelanteile des Differenzspektrums. Durchgezogene Linie: Anpassung der Differenzpunkte zu $\dot{M}=10^{16} \mathrm{~g} \mathrm{~s}^{-1}$ wie in Abbildung 4.3. Winkelanteile der Differenzbildung für $\vartheta=80^{\circ}$ (gestrichelte Linie, a) und für $\vartheta=5^{\circ}$ (gepunktete Linie, b).

Abbildung 4.4 die Spektren zu den Winkeln $\vartheta=80^{\circ}$ bzw. $\vartheta=5^{\circ}$ aufgetragen, die sich aus den gleichen gewichteten Anteilen für die Massenstromdichten zusammensetzen wie sie in der obigen Anpassung verwendet wurden, allerdings berechnet zu den jeweiligen Winkeln. Ein Vergleich mit den Daten von Priedhorsky et al. (1978; Fig. 4) zu den Phasen $\phi_{\text {mag }} \approx 0,8$ bzw. $\approx 0,6$ zeigt die qualitative Übereinstimmung der Verläufe. Das beobachtete Spektrum zu $\phi_{\text {mag }} \approx 0,8$ (hoher Sichtwinkel) liegt im Bereich $\lambda=0,43 \ldots 1,0 \mu \mathrm{m}$ deutlich über dem Verlauf zu $\phi_{\mathrm{mag}} \approx 0,6$ (kleiner Sichtwinkel); ebenso liegt der synthetische Fluß zu $\vartheta=80^{\circ}$ in diesem Bereich über dem zu $\vartheta=5^{\circ}$. Erst für Wellenlängen $\lambda>1,3 \mu \mathrm{m}$ werden die beobachteten Flüsse der beiden Phasen ähnlich groß; entsprechend ist der Verlauf in den gewichteten Spektren. Die bei der Anpassung des Differenzspektrums gefundenen Winkel der beiden Phasen sind also konsistent mit der Beobachtung. Die Werte $\vartheta=5^{\circ}$ bei $\phi_{\text {mag }} \approx 0,6$ und $\vartheta=80^{\circ}$ bei $\phi_{\text {mag }} \approx 0,8$ sind darüberhinaus verträglich mit den zur Erzeugung der optischen Lichtkurve in Kapitel 4.1 benutzten Werten für Inklination $i=50^{\circ}$ und Lage des Akkretionsflecks zum Pol $\beta=55^{\circ}$. Tatsächlich konnte mit dieser Vergleichsmethode des Flusses zu den Einzelwinkeln die in der Lichtkurve noch vertretbare Kombination $i=35^{\circ}$ und $\beta=70^{\circ}$ ausgeschlossen werden. Diese Geome- 
trie würde für $\phi_{\text {mag }} \approx 0,8$ zwar ebenfalls einen Sichtwinkel $\vartheta \approx 80^{\circ}$ ergeben, der Wert zu der Phase $\phi_{\text {mag }} \approx 0,6$ betrüge jedoch $\vartheta=35^{\circ}$. Ein Differenzspektrum kann konstruiert werden, das die Beobachtungsdaten hinreichend gut darstellt, aber die Einzelspektren zu den beiden Winkeln liefern einen Fluß, der die Beobachtung weit übersteigt.

Neben der Zyklotronstrahlung mit spektralem Maximum im Infraroten strahlt AM Her im harten Röntgenbereich als helle Quelle, wobei der beobachtete spektrale Verlauf sich gut mit Bremstrahlung zu hohen Temperaturen $\left(k_{\mathrm{B}} T_{\mathrm{ff}}=10 \ldots 20 \mathrm{keV}\right)$ modellieren läßt. Eine Beschreibung der Gesamtenergieverteilung des Polars muß daher sowohl die infrarote Zyklotronstrahlung wie auch die Röntgenemission konsistent erfassen.

Im Röntgenbereich liegt eine pointierte ROSAT-Beobachtung während eines „highstate" im April 1991 vor, die eine über einen Orbit gemittelte Zählrate im harten Röntgenbereich $(h \nu=0,5 \ldots 2,4 \mathrm{keV})$ von $1,3 \mathrm{cts} / \mathrm{s}$ liefert. Eine Anpassung an das mittlere Zählratenspektrum mit einem isothermen Bremsstrahlungsanteil zu $k_{\mathrm{B}} T_{\mathrm{ff}}=20 \mathrm{keV}$ liefert einen Fluß von 5,56 $10^{-3}$ Photonen $\mathrm{s}^{-1} \mathrm{~cm}^{-2} \mathrm{keV}^{-1}$ bei $h \nu=1 \mathrm{keV}$. Die Zählrate zur hellen Röntgenphase $\left(\phi_{\text {mag }} \approx 0,6\right)$ beträgt dagegen $2 \mathrm{cts} / \mathrm{s}$, entsprechend einem Photonenfluß 8, 55 $10^{-3}$ Photonen $\mathrm{s}^{-1} \mathrm{~cm}^{-2} \mathrm{keV}^{-1}$, was einem Energiefluß von $F_{\nu}=$ $5,67 \cdot 10^{-29} \mathrm{erg} \mathrm{s}^{-1} \mathrm{~cm}^{-2} \mathrm{~Hz}^{-1}$ bei $\nu=2,42 \cdot 10^{17} \mathrm{~Hz}$ entspricht. Dieser Datenpunkt ist zusammen mit den abgeschätzten Fehlern in Abbildung 4.5 (folgende Seite) dargestellt. Als spektraler Verlauf der harten Röntgenstrahlung kann in guter Näherung ein isothermes Bremsstrahlungsspektrum gewählt werden; neuere Anpassungen an eine spektral hochaufgelöste Beobachtung eines "high-state" mit dem Satelliten ASCA legen für den Temperaturbereich $k_{\mathrm{B}} T_{\mathrm{ff}}=10 \ldots 21 \mathrm{keV}$ nahe (Ishida et al. 1997). Die in Abbildung 4.5 eingetragenen Bremsstrahlungsspektren stellen somit den Bereich der Beobachtungsdaten eines ,high-state" dar.

Bei der Anpassung des infraroten-optischen Spektrums in Abbildung 1.3 wurde festgestellt, daß die hohen Massenstromdichten $\dot{m}>3 \mathrm{~g} \mathrm{~cm}^{-2} \mathrm{~s}^{-1}$ in diesem Wellenlängenbereich kaum noch zum beobachteten Fluß beitragen. Betrachtet man dagegen in Abbildung 4.5 die Flußverläufe im Röntgenbereich dieser Anpassungen, ergeben sich sichtbare Unterschiede zwischen den Spektren zu den beiden Akkretionsraten $\dot{M}=5 \cdot 10^{15}$ bzw. $10^{16} \mathrm{~g} \mathrm{~s}^{-1}$ : Die höhere Akkretionsrate erzeugt einen größeren Fluß über den gesamten Spektralbereich $\nu>10^{15} \mathrm{~Hz}$, da sie auch mehr Energie in die Emissionsregion einbringt als die niedrige Akkretionsrate. Im Infraroten-Optischen sind dagegen keine wesentlichen Differenzen zwischen den beiden Akkretionsraten festzustellen. Die Beiträge der hohen Massenstromdichten liegen daher hauptsächlich im Röntgenbereich.

Um den durch die Bremsstrahlungskurven beschriebenen beobachteten Fluß für $\nu>$ $10^{19} \mathrm{~Hz}$ wiederzugeben, ist die Anwesenheit der $\dot{m}>3 \mathrm{~g} \mathrm{~cm}^{-2} \mathrm{~s}^{-1}$ in der Gewichtung der einzelnen Massenstromdichten notwendig. Allerdings konnte keine Gewichtung gefunden werden, in der sowohl der ROSAT-Datenpunkt als auch der Verlauf der Bremsstrahlungskurven übereinstimmend angepasst werden: Ein Spektrum, das den ROSAT-Fluß erzeugt, reicht nicht zu höheren Frequenzen hin, während eine annähernde Darstellung des Bremsstrahlungsflusses erheblich über dem ROSAT-Datenpunkt liegt, wie in Abbildung 4.5 deutlich zu erkennen ist. 


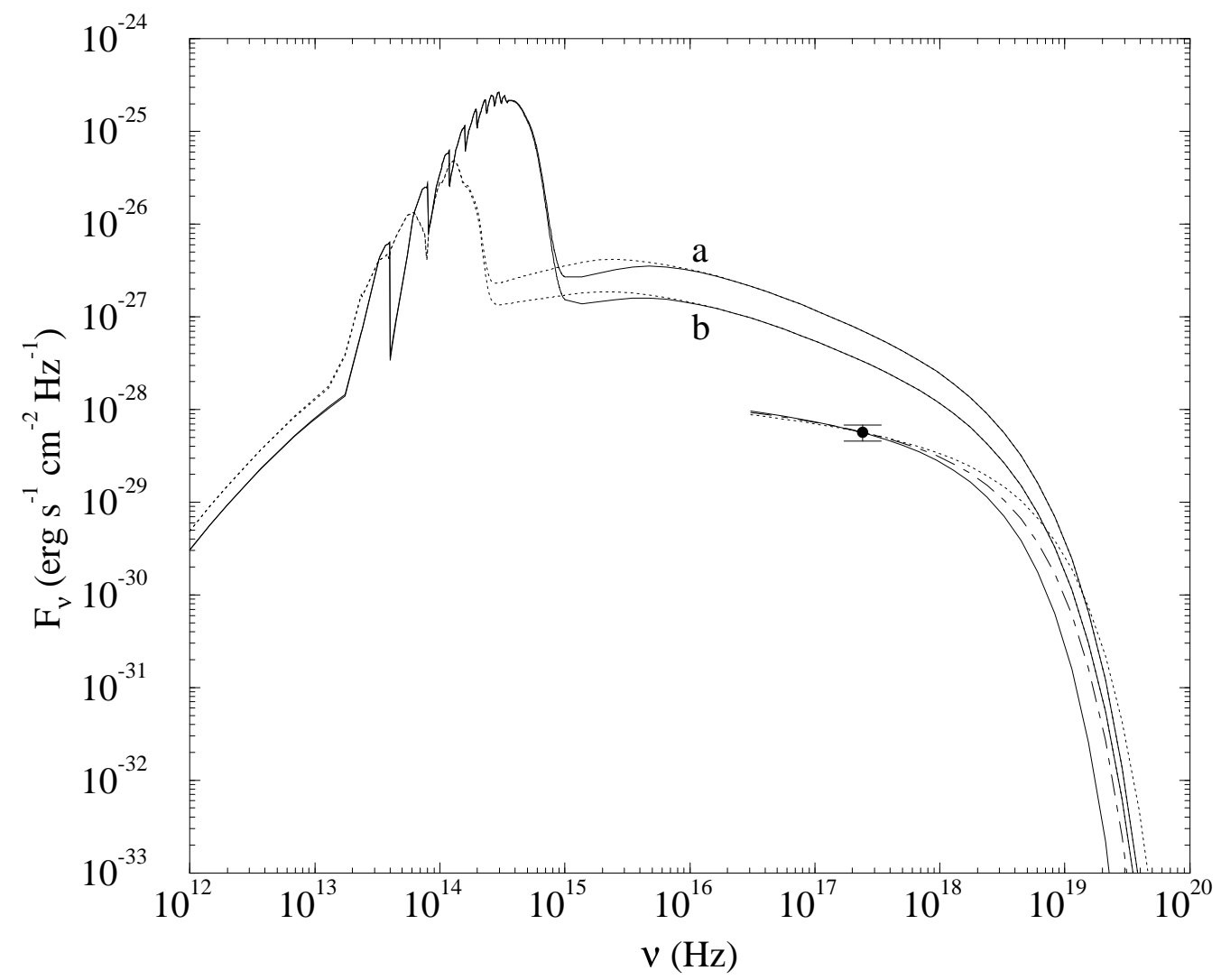

Abbildung 4.5: Gesamtspektrum der Anpassungen aus Abb. 4.3. Dargestellt sind die Spektren zu der Akkretionsrate $\dot{M}=10^{16} \mathrm{~g} \mathrm{~s}^{-1}$ (a), sowie zu $\dot{M}=5 \cdot 10^{15} \mathrm{~g} \mathrm{~s}^{-1}$ (b) für die zwei Winkel der Differenzbildung $\vartheta=5^{\circ}$ (gepunktete Linien) bzw. $\vartheta=80^{\circ}$ (durchgezogene Linien). Eingetragen ist weiterhin der Datenpunkt einer ROSAT-Beobachtung im April 1991 ( „high-state“), sowie isotherme Bremsstrahlungsspektren zu $k_{\mathrm{B}} T_{\mathrm{ff}}=10 \mathrm{keV}$ (durchgezogene Linie), $14 \mathrm{keV}$ (strich-punktierte Linie), bzw. $21 \mathrm{keV}$ (gepunktete Linie), die als Bereich der spektralen Verläufe der Beobachtungen dienen.

Eine einfache Abschätzung der dynamischen Verhältnisse in der Atmosphäre des weißen Zwergs bei der Akkretion mit hohen Massenstromdichten liefert, daß für $\dot{m}>$ $10 \mathrm{~g} \mathrm{~cm}^{-2} \mathrm{~s}^{-1}$ der Staudruck am Ende der Akkretionssäule durch den Atmosphärendruck des weißen Zwergs in Bereichen ausgeglichen wird, in denen die umgebende Säulendichte des Materials $N_{\mathrm{H}}>10^{25} \mathrm{~cm}^{-2}$ ist (Beuermann 1998). Der Staudruck des akkretierten Materials senkt somit den Stoß soweit in die Sternatmosphäre ein, daß der Stoß unterhalb der Photosphäre liegt, und die Röntgenstrahlung der Akkretionssäule nicht ohne starke Photoabsorption entweichen kann (Abb. 4.6). Die Ionisationsverhältnisse in der über dem Stoß liegenden einfallenden Materie können in guter Näherung durch ein photoionisiertes Gas mit Ionisationsparameter $\Xi:=L /\left(4 \pi c r^{2} N_{\mathrm{H}} k_{\mathrm{B}} T\right) \approx 10$ - wenn $L$ die umgebende Leuchtkraft, $r$ eine typische Länge und die Temperatur $T \approx 10^{5} \mathrm{~K}$ ist - beschrieben werden, bzw. mit einem Gas im koronalen Ionisationsgleichgewicht zur Temperatur $T=10^{6} \mathrm{~K}$, in dem Ionisationen und Rekombinationen im thermischen Gleichgewicht stehen (Krolik \& 


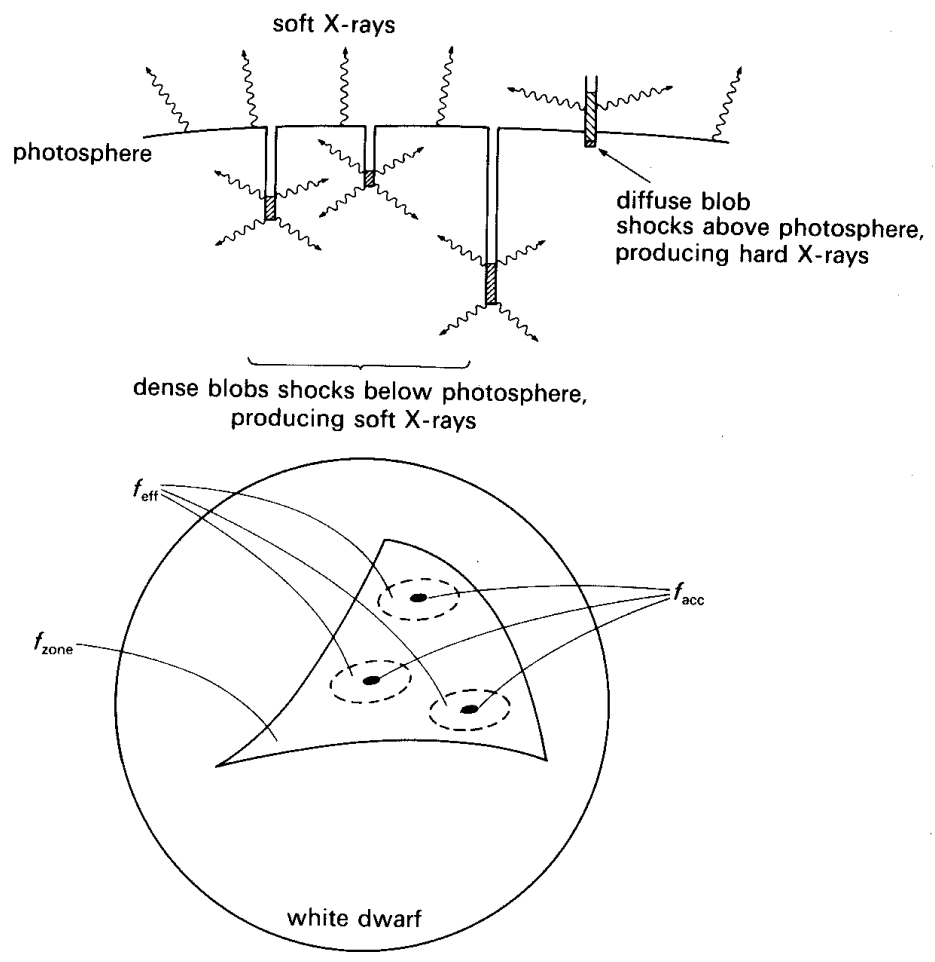

\begin{abstract}
Abbildung 4.6: Schematische Darstellung der Einsenkung in die Photosphäre des weißen Zwergs von Teilen der Akkretionsregion mit hoher Massenstromdichte (aus Frank, King \& Raine 1992). (Oben) Dargestellt sind mehrere Bereiche mit hohem $\dot{m}$, deren Staudruck so groß ist, daß sie in die Photosphäre einsinken, und deren Stoßhöhe so niedrig ist, daß die Strahlung der Emissisonregion (schattiert dargestellt) vollständig von der Photosphäre absorbiert wird und weiche Röntgenstrahlung erzeugen. (Unten) Flächenanteile der Akkretion. $f_{\text {acc }}$ : Fläche der einzelnen Massenstromdichte, $f_{\text {eff }}$ : Fläche der reprozessierten Schwarzkörperstrahlung, $f_{\text {zone: }}$ zur Erzeugung einer Lichtkurve im weichen Röntgenbereich nötige Fläche.
\end{abstract}

Kallman 1984). Der Wirkungsquerschnitt der Absorption wurde in einer einfachen Form angewendet, in der keine Absorption für $h \nu<0,28 \mathrm{keV}$ stattfindet und für $h \nu>7 \mathrm{keV}$ eine Proportionalität zu $\nu^{-3}$ angenommen wird. Im Zwischenbereich sind die Kanten der wichtigsten Elemente berücksichtigt (O VI, O VII, Ne(K), Fe(L) und $\mathrm{Fe}(\mathrm{K})$ ).

In Abbildung 4.7 (folgende Seite) ist eine Anpassung an den Bremsstrahlungsverlauf zu $k_{\mathrm{B}} T_{\mathrm{ff}} \approx 14 \mathrm{keV}$ unter Berücksichtigung der Absorption dargestellt. Dabei wurde für die Massenstromdichte $\dot{m}=100 \mathrm{~g} \mathrm{~cm}^{-2} \mathrm{~s}^{-1}$ eine Säulendichte $N_{\mathrm{H}} \approx 5 \cdot 10^{25} \mathrm{~cm}^{-2}$ und für $\dot{m}=10 \mathrm{~g} \mathrm{~cm}^{-2} \mathrm{~s}^{-1}$ ein $N_{\mathrm{H}} \approx 3 \cdot 10^{23} \mathrm{~cm}^{-2}$ verwendet. Die folgenden kleineren Massenstromdichten werden mit jeweils 1/10 der vorhergehenden Säulendichte absorbiert. Diese Skalierung erscheint plausibel, da für kleine $\dot{m}$ kaum noch Einsenkung stattfindet, und die Röntgenstrahlung nur wenig durch die einfallende Materie vor dem Stoß abgeschwächt wird. Zur Veranschaulichung der Auswirkung der Absorption auf die Spektren, ist in Abbildung 4.8 (S. 93) der Beitrag der einzelnen Massenstromdichten zum 


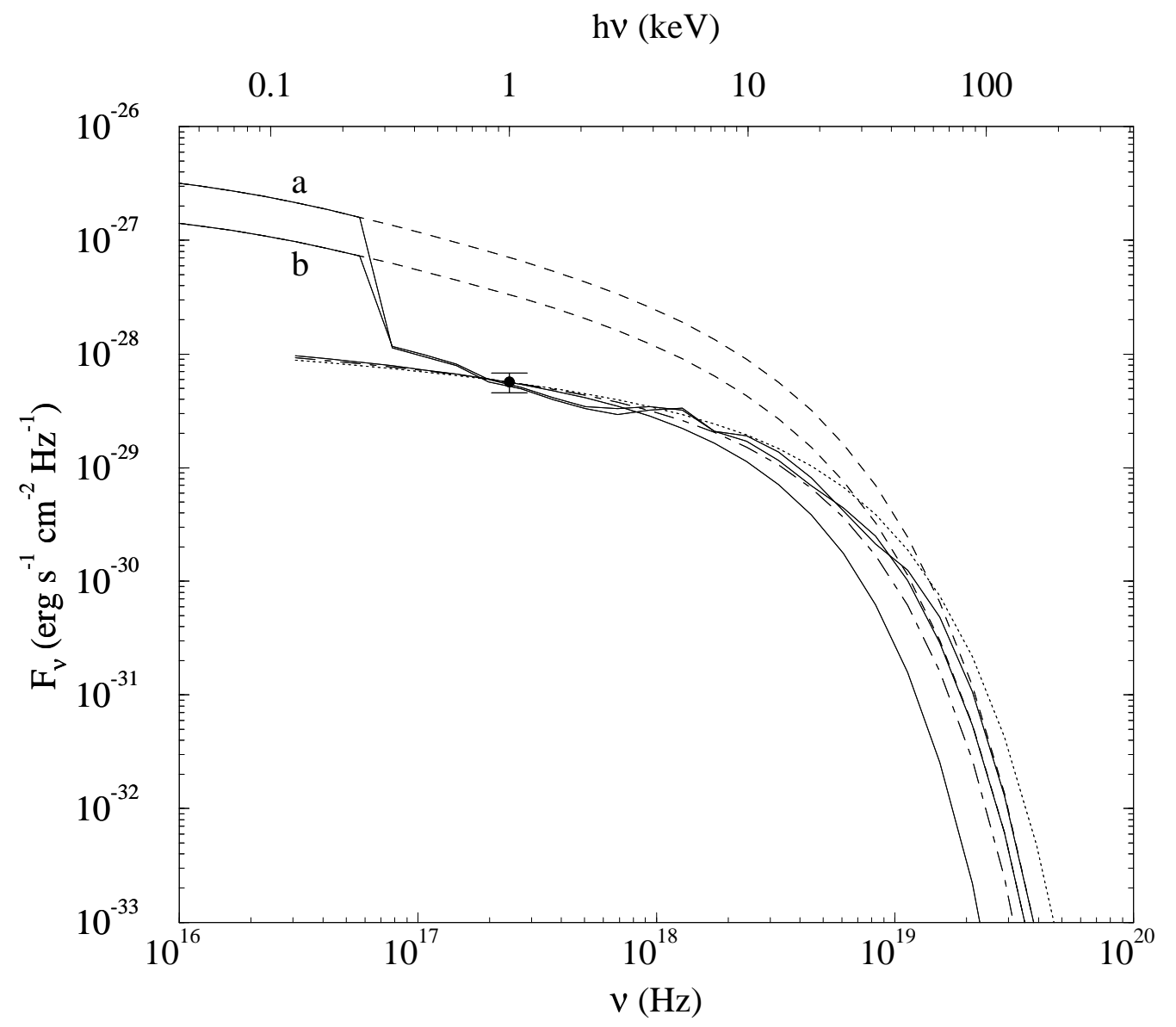

Abbildung 4.7: Anpassung des Bremsstrahlungsverlaufs nach Absorption von Teilen der Röntgenstrahlung in der Atmosphäre des weißen Zwergs. Dargestellt sind die absorbierten Flüsse (durchgezogene Linien) für $\dot{M}=10^{16} \mathrm{~g} \mathrm{~s}^{-1}$ (a), bzw. $5 \cdot 10^{15} \mathrm{~g} \mathrm{~s}^{-1}$ (b) und die entsprechenden unabsorbierten Flüsse (gestrichelte Linien). Weiterhin der ROSAT-Datenpunkt und die Bremsstrahlungskurven zu $k_{\mathrm{B}} T_{\mathrm{ff}}=10 \mathrm{keV}$ (durchgezogene Linie), $14 \mathrm{keV}$ (strich-punktierte Linie), bzw. $21 \mathrm{keV}$ (gepunktete Linie).

Gesamtspektrum nach der Abschwächung dargestellt. Die höchste Massenstromdichte von $\dot{m}=100 \mathrm{~g} \mathrm{~cm}^{-2} \mathrm{~s}^{-1}$ erleidet auch die stärkste Absorption, so daß die resultierenden Beiträge auf den Frequenzbereich $\nu>10^{19} \mathrm{~Hz}(h \nu>40 \mathrm{keV})$ beschränkt werden, dieses $\dot{m}$ also nur im hochenergetischen Teil des Spektrums wesentlich wird. Durch die geringere Absorption für $\dot{m}=10 \mathrm{~g} \mathrm{~cm}^{-2} \mathrm{~s}^{-1}$, ist deren Beitrag in den Bereichen zwischen $\nu \approx 10^{18} \mathrm{~Hz}$ und $\nu \approx 10^{19} \mathrm{~Hz}$ zu finden. Die Massenstromdichte $\dot{m}=1 \mathrm{~g} \mathrm{~cm}^{-2} \mathrm{~s}^{-1}$ ist nur noch unwesentlich abgeschwächt und liefert den Hauptbeitrag zu den Frequenzen um den ROSAT-Datenpunkt. Die folgenden $\dot{m}$ sind unabsorbiert, tragen aber kaum noch zum Röntgenspektrum bei; ihr Hauptbeitrag zum Gesamtspektrum liegt im Optischen bzw. Infraroten. Damit läßt sich durch die sukzessive Absorption von Teilen der Röntgenstrahlung der einzelnen Beiträge der Massenstromdichten in einem gewichteten Gesamtspek- 


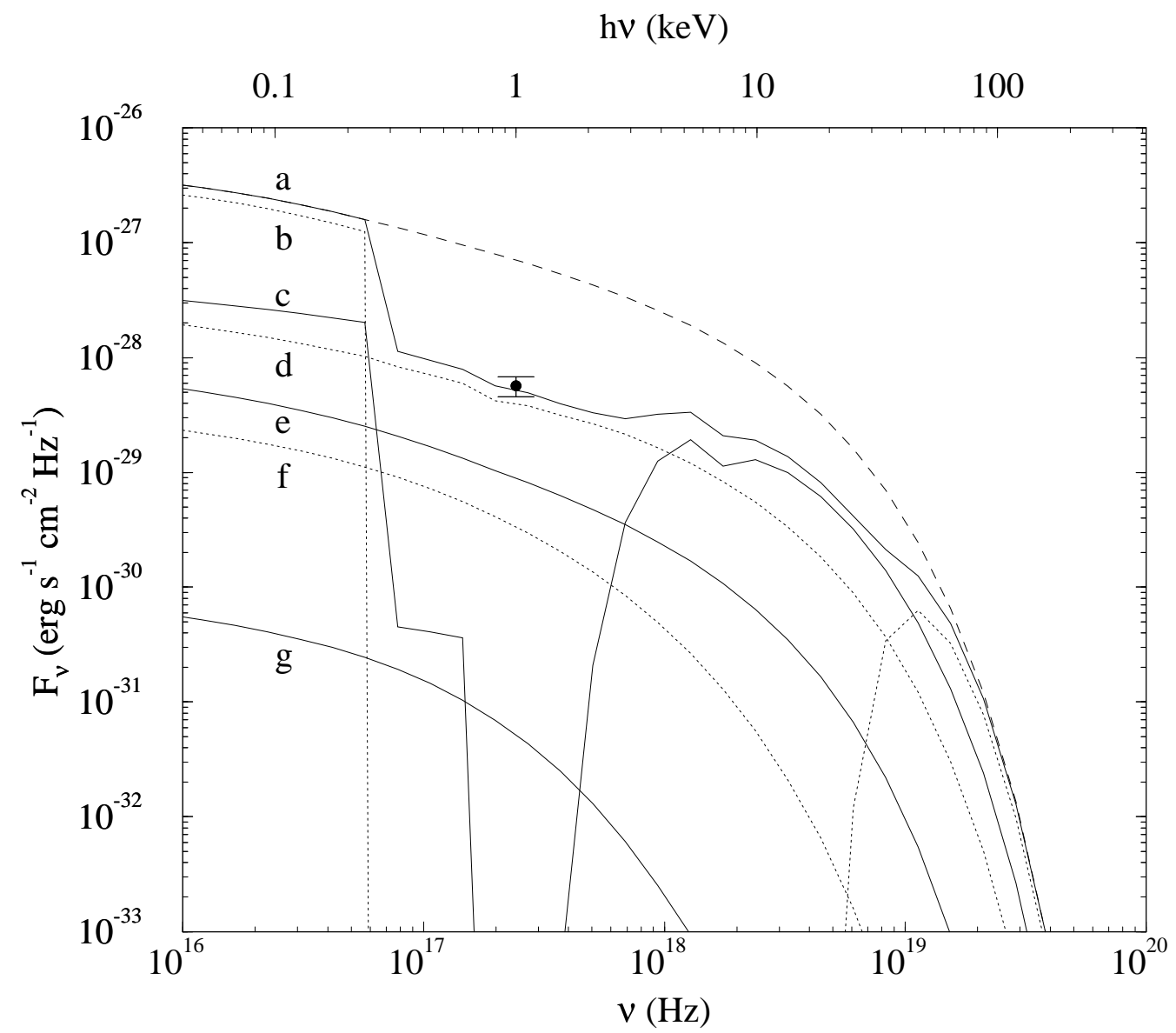

Abbildung 4.8: Anteile der einzelnen Massenstromdichten am absorbierten Gesamtspektrum zu $\dot{M}=10^{16} \mathrm{~g} \mathrm{~s}^{-1}$ aus Abb. 4.7. Dargestellt sind: (a) Unabsorbierter (gestrichelte Linie) bzw. absorbierter Gesamtfluß (durchgezogene Linie), sowie die Anteile der Massenstromdichten (b) $\dot{m}=100$, (c) 10 , (d) 1 , (e) $10^{-1}$, (f) $10^{-2}$ und (g) $10^{-3} \mathrm{~g} \mathrm{~cm}^{-2} \mathrm{~s}^{-1}$.

trum ein Flußverlauf erzeugen, der sowohl die harte Röntgenstrahlung als auch deren weichen Bereich richtig wiedergibt, wie im folgenden gezeigt wird.

Die beschriebene Absorption der Röntgenstrahlung durch das die Akkretionssäule umgebende Material kann nur ein energetisch konsistentes Modell sein, wenn der einbehaltene Strahlungsfluß in Form einer anderen Komponente im Spektrum wieder auftaucht. Aus der Vorstellung der absorbierenden Prozesse und Thermalisierung der einbehaltenen Strahlungsenergie in der Atmosphäre, erwartet man eine anähernd schwarzkörperartige Strahlung, deren Energieinhalt dem absorbierten Fluß entspricht.

Nimmt man an, daß die absorbierte Bremsstrahlung in den Halbraum der Sternoberfläche gestrahlt wird und die erwartete reprozessierte weiche Röntgenstrahlung wie ein Schwarzkörper von einer ebenen Fläche abgestrahlt wird, dann führt Energieerhaltung in 


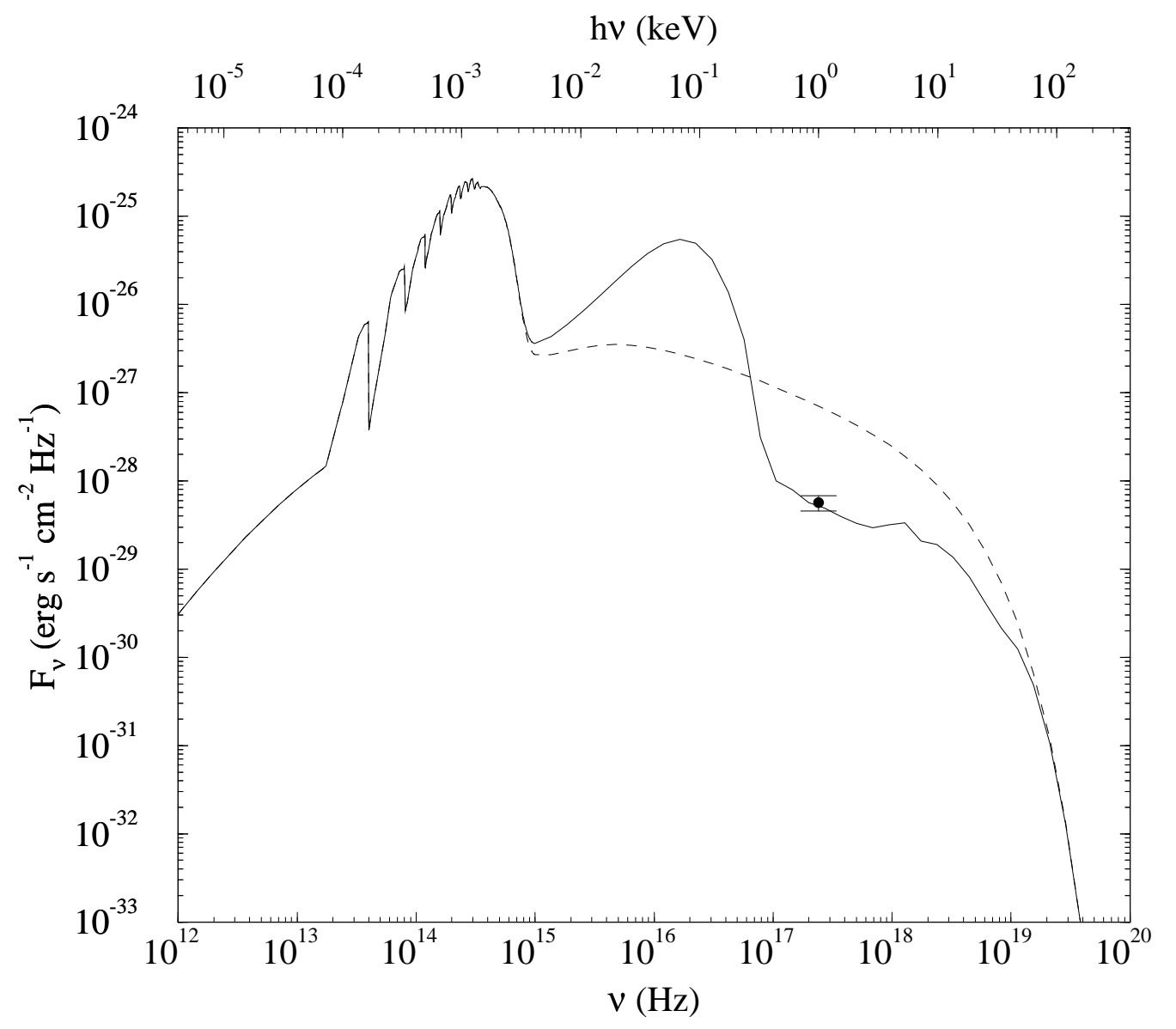

Abbildung 4.9: Schwarzkörperkomponente aus der bei der Absorption einbehalteten Strahlungsenergie. Dargstellt ist der unabsorbierte Fluß zu der Akkretionsrate $\dot{M}=10^{16} \mathrm{~g} \mathrm{~s}^{-1}$ (gestrichelte Linie) sowie das durch Absorption abgeschwächte Spektrum mit der reprozessierten Schwarzkörperkomponente zu $k_{\mathrm{B}} T_{\mathrm{bb}} \approx 25 \mathrm{eV}$.

Form der Identität der Leuchtkräfte auf folgenden Ausdruck für die Effektivtemperatur des Schwarzkörpers:

$$
T_{\mathrm{bb}}^{4}=\frac{2 \pi d^{2} f_{\mathrm{abs}}}{A_{\mathrm{bb}} \sigma},
$$

mit $f_{\text {abs }}$ dem absorbierten Strahlungsfluß, $A_{\mathrm{bb}}$ der Fläche des Schwarkörperstrahlers, $d$ der Entfernung des Sterns und $\sigma$ der Stefan-Boltzmann-Konstante. Für das Spektrum zu der Akkretionsrate $\dot{M}=10^{16} \mathrm{~g} \mathrm{~s}^{-1}$ erhält man einen Wert für den frequenzintegrierten, absorbierten Fluß von $f_{\text {abs }}=7,2 \cdot 10^{-10} \mathrm{erg} \mathrm{s}^{-1} \mathrm{~cm}^{-2}$. Die Temperatur des resultierenden Schwarzkörpers ist nun festgelegt, wenn eine Annahme über dessen strahlende Fläche gemacht wird. Als plausibel erscheint hier mindestens die Flächenanteile aus der Gewichtung der absorbierten Massenstromdichten anzunehmen. Realistischerweise wird die strahlende Region jedoch größer sein, da durch Transportprozesse (Strahlung, Wärmeleitung) die absorbierte Energie weiter verteilt sein wird. Zum Beispiel erscheint es sinnvoll, eine radiale Ausdehnung entsprechend der Tiefe der Einsenkung der Stoßregion 
in der Atmosphäre anzunehmen. Ein Geometriefaktor von $2 \ldots 3$ erscheint dann angebracht. Damit ergibt sich für den obigen absorbierten Fluß $f_{\text {abs }}$ im Fall der Akkretionsrate $\dot{M}=10^{16} \mathrm{~g} \mathrm{~s}^{-1}$ ein Schwarzkörperspektrum der Temperatur $k_{\mathrm{B}} T_{\mathrm{bb}} \approx 25 \mathrm{eV}$ mit einem Fluß $f_{\mathrm{bb}}=1,5 \cdot 10^{-9} \mathrm{erg} \mathrm{s}^{-1} \mathrm{~cm}^{-2}$. Diese Werte sind konsistent mit aus Beobachtungen abgeleiteten Werten und bestätigen die Modellannahmen. In Abbildung 4.9 ist die aus der absorbierten Bremsstrahlung entstandene Schwarzkörperkomponente zusammen mit dem unabsorbierten Fluß dargestellt. Eine entsprechende Rechnung für die Akkretionsrate $\dot{M}=5 \cdot 10^{15} \mathrm{~g} \mathrm{~s}^{-1}$ ergibt einen geringeren Schwarzkörperanteil und kann nicht mit der Beobachtung in Einklang gebracht werden. Dieser Wert für $\dot{M}$ erscheint daher als zu gering, um einen ,high-state" des Polars AM Her zu beschreiben.

Um den Energieinhalt der im Spektrum enthaltenen Komponenten zu verdeutlichen, ist in Abbildung 4.10 (folgende Seite) eine Darstellung $\nu F_{\nu}$ gegen $\nu$ gewählt, in der gleiche Flächen unter der Kurve gleichen Energieinhalten entsprechen. Darin wird der am Anfang des Kapitels angesprochene Überschuß an weicher Röntgenstrahlung sichtbar: Die Schwarzkörperkomponente überwiegt sowohl die Zyklotronstrahlung wie auch die nicht absorbierte Bremsstrahlung. Um das berechnete Gesamtspektrum mit der Beobachtung vergleichen zu können, sind in Abbildung 4.11 (S. 97) Daten aus verschiedenen Frequenzbereichen zusammengefaßt, die die einzelnen Beiträge der Systemkomponenten weißer Zwerg, Sekundärstern und Akkretionstrom beeinhalten.

Mit dem Modell der Strahlung einer stationären Akkretionssäule und der Gewichtung der Strahlungsbeiträge eines weiten Bereichs von Massenstromdichten kann somit das Gesamtspektrum des Polars AM Her wiedergegeben werden. Dabei ergibt sich weiterhin eine natürliche Erklärung der Komponente der weichen Röntgenstrahlung als reprozessierte Energie eines Teils der von den hohen Massenstromdichten gelieferten und in der Atmosphäre des weißen Zwergs absorbierten Strahlung. Eine definitive Schlußfolgerung ist die, daß die strukturierte Akkretionsregion Massenstromdichten aufweist, die über mindestens sechs Zehnerpotenzen variieren.

\subsection{2 "Low-state" Zustand des Systems}

Neben dem leuchtkräftigen Zustand von AM Her befindet sich das System in unregelmäßigen Abständen in einer Phase langanhaltender geringer Helligkeit, dem sog. „low-state“. In diesem Zustand ist der optische Strahlungsfluß um einen Faktor $\approx 4$ geringer als im „high-state“, während der harte Röntgenfluß bis auf Null heruntergehen kann. Zur Erklärung dieser Veränderungen wird i. allg. angenommen, daß sich das System in einem Zustand geringer Akkretionstätigkeit befindet, wobei die verbleibende optische Helligkeit von der photosphärischen Strahlung des weißen Zwergs und des Sekundärsterns stammt. Speziell in AM Her kam die Akkretion bisher nie vollständig zum Erliegen, denn AM Her wurde niemals ohne einen Rest von Röntgenstrahlung beobachtet.

Eine offene Frage ist in diesem Zusammenhang, ob während eines „low-state" eine wenn auch schwache - Schwarzkörperkomponente im weichen Röntgenbereich vorhanden sein kann. Da die Möglichkeiten der direkten Beobachtung von weicher Röntgenstrahlung niedriger Intensität jedoch begrenzt sind, wäre eine Voraussage über die Bedingungen des 


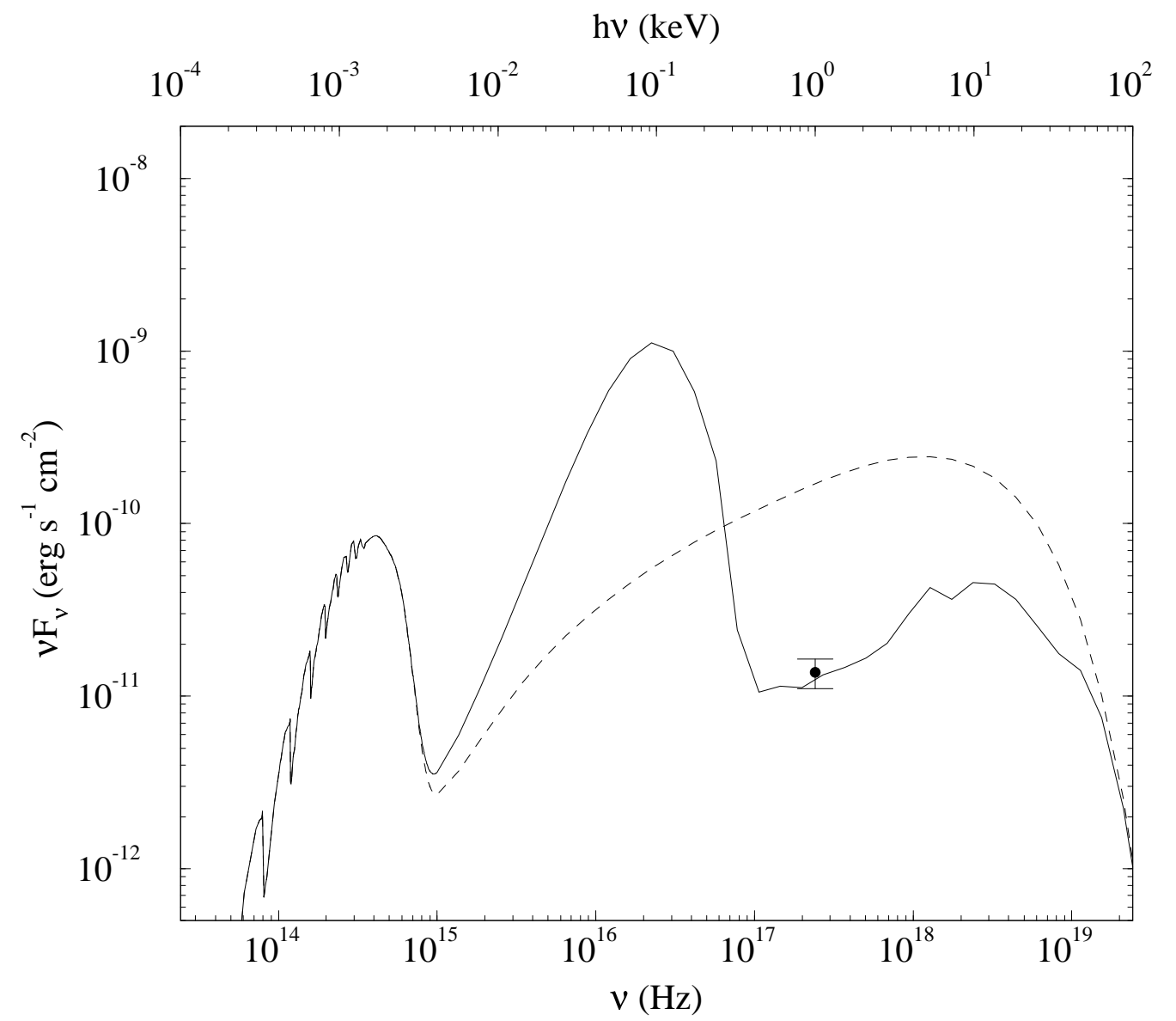

Abbildung 4.10: Darstellung des „high-state“-Energieinhalts der einzelnen Komponenten aus Abb. 4.9. Im Vergleich zu Abb. 4.11 ist zu beachten, daß hier die Beiträge aus der Emission des weißen Zwergs, des Sekundärsterns und des Akkretionsstroms nicht enthalten sind.

Auftretens einer solchen Komponente hilfreich, um z. B. Aussagen über die Akkretionsrate treffen zu können. In diesem Kapitel soll nun, analog zum Vorgehen in der Anpassung des „,high-state“-Gesamtspektrums, eine spektrale Energieverteilung für den „low-state“ durchgeführt werden, um zu einem generellen Verständnis des Systems zu kommen.

Im infraroten Teil des Gesamtspektrums liegen spektroskopische Beobachtungen von Bailey, Ferrario \& Wickramasinghe (1991) vor, die während eines „low-state" im Juni 1990 aufgenommen wurden. Die Autoren leiten aus ihren Beobachtungen ein mittleres Spektrum zu den Phasen $\phi_{\mathrm{mag}}=0,46 \ldots 0,88$, die das Helligkeitsmaximum im K-Band symmetrisch umschließen (Bailey, Hough \& Wickramasinghe 1988), her. Dazu subtrahieren sie den beobachteten Fluß zu den Phasen $\phi_{\text {mag }}=0,0 \ldots 0,35$ von dem zu $\phi_{\operatorname{mag}}=0,46 \ldots 0,88$. Damit das resultierende Spektrum als reines Zyklotronspektrum (bei vorausgesetzter Phasenkonstanz der übrigen Komponenten) aufgefasst werden kann, darf zu $\phi_{\text {mag }}=0,0 \ldots 0,35$ kein Anteil dieser Strahlung vorhanden sein. Die Autoren geben eine Anpassung des Spektrums mit einem Modellspektrum eines M-Sterns an, der 


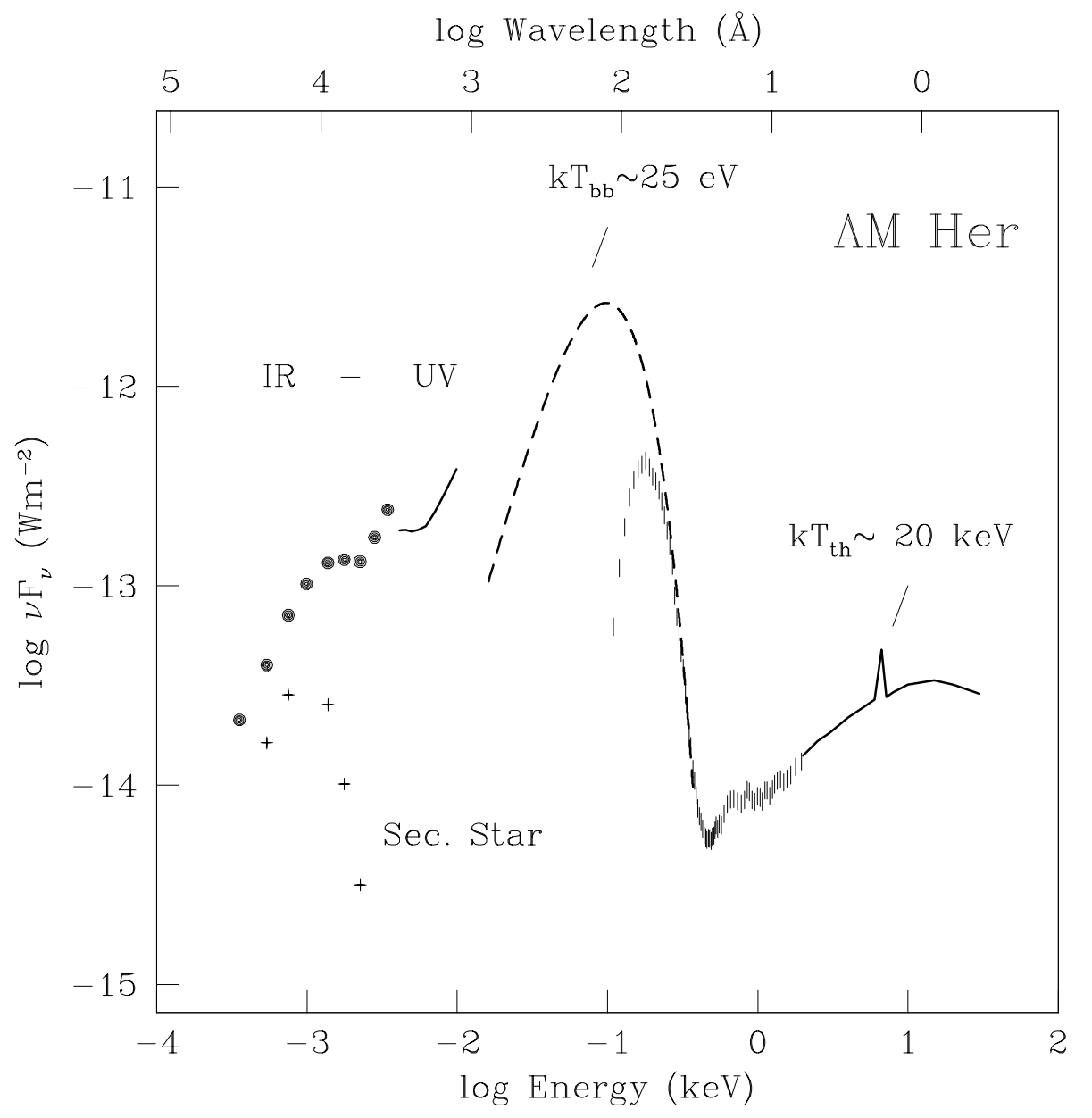

\begin{abstract}
Abbildung 4.11: Zusammenstellung verschiedener Beobachtungsdaten für den „high-state“ des Polars AM Her (aus Beuermann 1998). Dargestellt sind die Beiträge ( $\mathrm{W} \mathrm{m}^{-2}=10^{3} \mathrm{erg} \mathrm{s}^{-1} \mathrm{~cm}^{-2}$ ) aus Bremsstrahlung zu $k_{\mathrm{B}} T_{\mathrm{ff}} \approx 20 \mathrm{keV}$, Schwarzkörperkomponente zu $k_{\mathrm{B}} T_{\mathrm{bb}} \approx 25 \mathrm{eV}$, Zyklotronstrahlung (IR), weißem Zwerg und Akkretionsstrom (UV) sowie Sekundärstern (Sec. Star).
\end{abstract}

den zu diesen Phasen sichtbaren Sekundärstern wiedergeben soll. Tatsächlich zeigt diese Anpassung jedoch Abweichungen, die Bailey et al. als möglichen Anteil aus Zyklotronstrahlung gedeutet hatten. Ich schließe diese Möglichkeit jedoch aus, weil das beobachtete Spektrum sehr gut zu modernen Infrarotspektren von M-Sternen des Spektraltyps dM4 paßt (z. B. GL268; Leggett et al. 1996). Dies ist auch konsistent mit dem von Beuermann et al. (1998) gefundenen Spektraltyp des Begleiters in AM Herculis. Das Spektrum zu $\phi_{\mathrm{mag}}=0,0 \ldots 0,35$ zeigt also besser den Beitrag des Sekundärsterns, als dies aus der in Bailey, Ferrario \& Wickramasinghe (1991) dargestellten Abbildung ersichtlich ist. Das aus der Subtraktion resultierende Spektrum ist in Abbildung 4.12 (folgende Seite) dargestellt und ist somit in guter Näherung ein reines Zyklotronspektrum des Phasenbereichs $\phi_{\text {mag }}=0,46 \ldots 0,88$. 


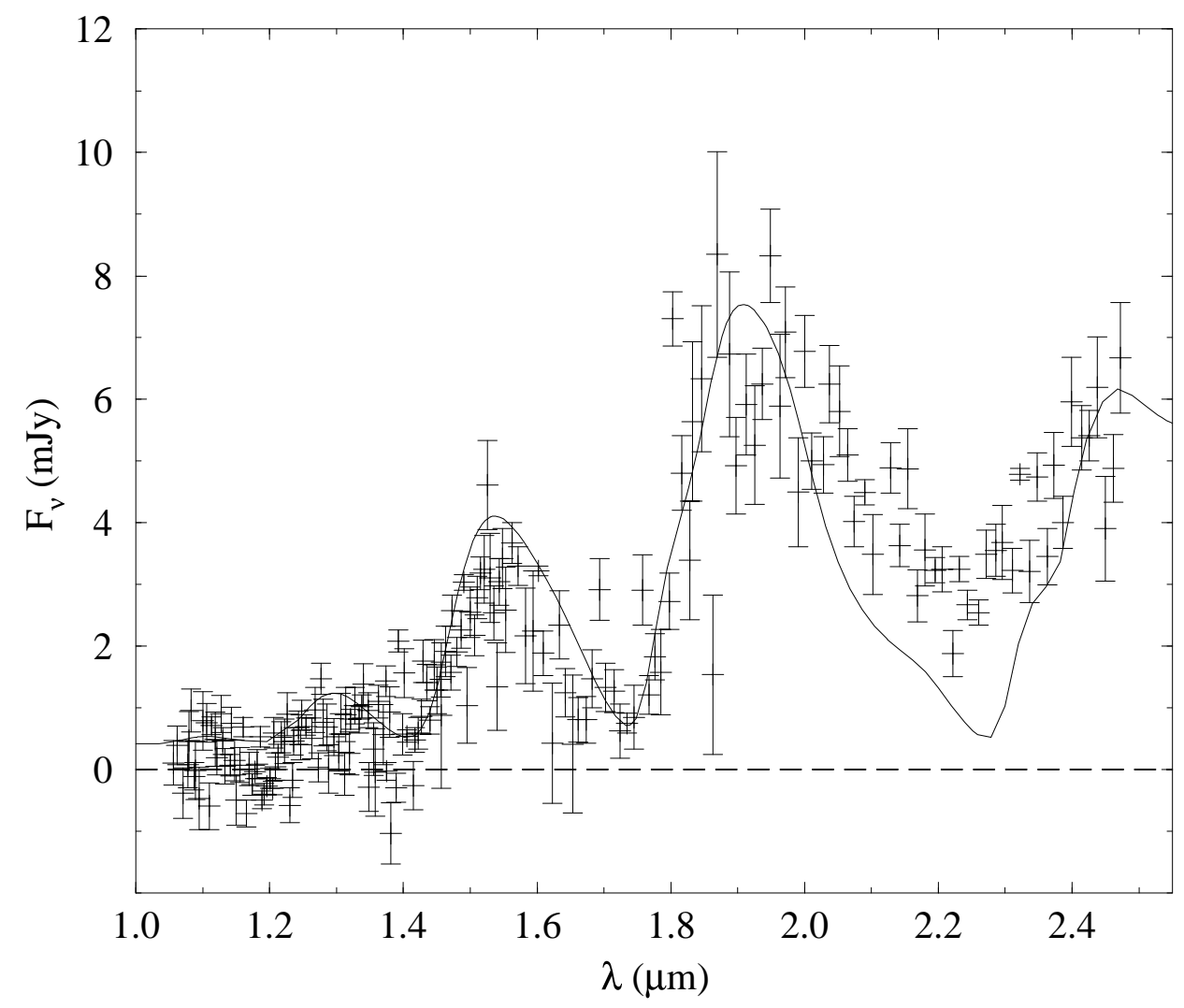

Abbildung 4.12: Mittleres Zyklotronspektrum zu den Phasen $\phi_{\mathrm{mag}}=0,46 \ldots 0,88$ für einen „low-state“ des Polars AM Her. Dargestellt sind spektroskopische Beobachtungsdaten aus dem Juni 1990 (aus Bailey, Ferrario \& Wickramasinghe 1991), sowie eine Anpassung mit einer Gewichtung der über einen Sichtwinkelbereich gemittelten Beiträge einzelner Massenstromdichten.

Für die Systemgeometrie wird hier (wie schon bei den Betrachtungen zum „highstate") eine Inklination $i=50^{\circ}$ und eine Lage des Akkretionsflecks zur Polachse des weißen Zwergs $\beta=55^{\circ}$ gewählt. Daraus ergibt sich eine Winkelüberdeckung von $\vartheta=5 \ldots 60^{\circ}$ währende der Phasen $\phi_{\text {mag }}=0,46 \ldots 0,88$. Eine Anpassung durch synthetische Zyklotronspektren muß daher, zusätzlich zu einer Gewichtung einzelner Massenstromdichten, eine Mittelung über den angegeben Sichtwinkelbereich berücksichtigen.

Als Datenpunkt im Röntgenbereich wird eine ROSAT-Beobachtung ( $R A S S$ Kampagne) während des „low-state“ im September 1990 verwendet. Die über den Orbit gemittelte Zählrate im Bereich $h \nu=0,1 \ldots 2,4 \mathrm{keV}$ beträgt 0,13 cts/s. Eine Anpassung an das Zählratenspektrum mit einem isothermen Bremsstrahlungsanteil zu $k_{\mathrm{B}} T_{\mathrm{ff}}=20 \mathrm{keV}$ liefert einen Fluß von 3, $7 \cdot 10^{-4}$ Photonen $\mathrm{s}^{-1} \mathrm{~cm}^{-2} \mathrm{keV}^{-1}$ bei $h \nu=1 \mathrm{keV}$, woraus sich ein Energiefluß von $F_{\nu}=2,4 \cdot 10^{-30} \mathrm{erg} \mathrm{s}^{-1} \mathrm{~cm}^{-2} \mathrm{~Hz}^{-1}$ bei $\nu=2,42 \cdot 10^{17} \mathrm{~Hz}$ ergibt. Um einen spektralen Verlauf der Beobachtung darzustellen, wurden isotherme Bremsstrahlungsspektren zu $k_{\mathrm{B}} T_{\mathrm{ff}}=5 \ldots 20 \mathrm{keV}$ benutzt. Dieser Temperaturbereich wird durch verschiedene Anpassungen an das Zählratenspektrum von ROSAT-Beobachtungen gerechtfertigt. Der so 


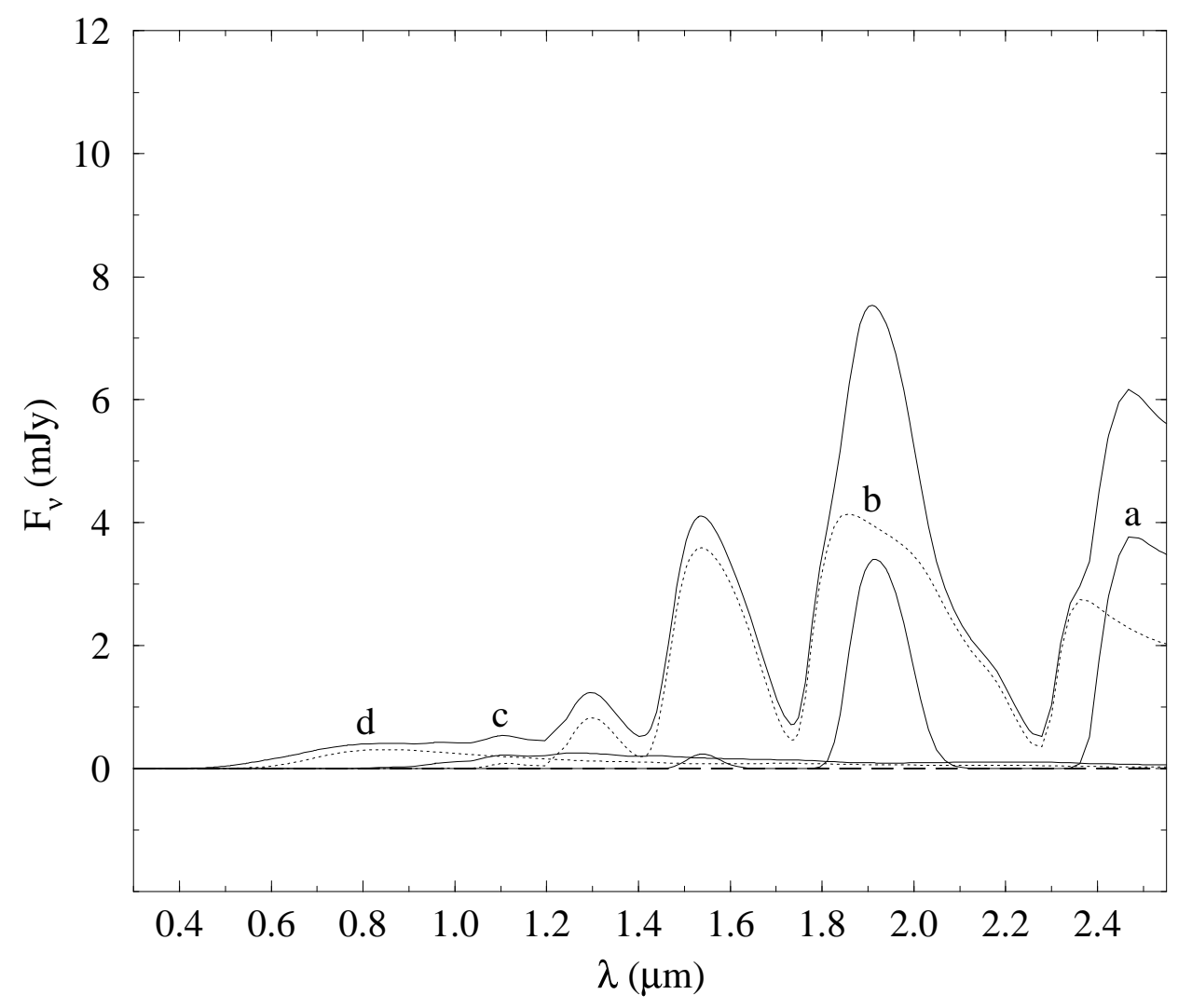

Abbildung 4.13: Anteile der einzelnen Massenstromdichten an der Anpassung aus Abbildung 4.12. Dargestellt ist die Anpassung (durchgezogene Linie) und die Teilspektren zu (a) $\dot{m}=10^{-4}$, (b) $10^{-3}$, (c) $10^{-2}$ und (d) $10^{-1} \mathrm{~g} \mathrm{~cm}^{-2} \mathrm{~s}^{-1}$. Die Beobachtungsdaten und die Anteile aus den höheren Massenstromdichten sind der Übersicht halber nicht aufgetragen. Zum Vergleich mit den High-state Daten ist die Abszisse wie in Abbildung 4.3 gewählt.

nachgebildete Bereich der Beobachtungen eines „low-state"-Spektrums im Röntgenbereich ist in Abbildung 4.14 (folgende Seite) angegeben.

Um den gesamten spektralen Verlauf der ,low-state"-Emission anzupassen, wurde versucht, sowohl die Infrarot-Daten wie auch die Röntgenflüsse gleichzeitig zu beschreiben. Im Röntgenbereich liegt kein beobachteter spektraler Verlauf vor, daher wurde für die Anpassung das Bremsstrahlungsspektrum zu $k_{\mathrm{B}} T_{\mathrm{ff}}=10 \mathrm{keV}$ ausgewählt. Als Durchschnittswert des von den Beobachtungen überspannten Bereichs der harten Röntgenstrahlung, scheint dieses Spektrum geeignet eine reale Beobachtung am ehesten nachzubilden. Da der Variationsbereich der Sichtwinkel für die Zyklotronstrahlung durch die Systemgeometrie vorgegeben ist, zielte die Anpassung auf die Gewichtung der einzelnen Massenstromdichten $\mathrm{ab}$, deren Strahlungsbeiträge jeweils über den Sichtwinkelbereich gemittelt wurden.

Eine Gewichtung, die sowohl den Verlauf der Infrarot-Beobachtung als auch den harten Röntgenanteil liefert, wurde durch Variation der Beiträge der einzelnen Massenstromdichten gefunden. Die resultierenden Flächengewichte $\Delta A_{\text {acc }}$ und der differentielle Beitrag $\Delta \dot{M}$ zur Akkretionsrate $\dot{M}$ sind in folgender Tabelle angegeben: 


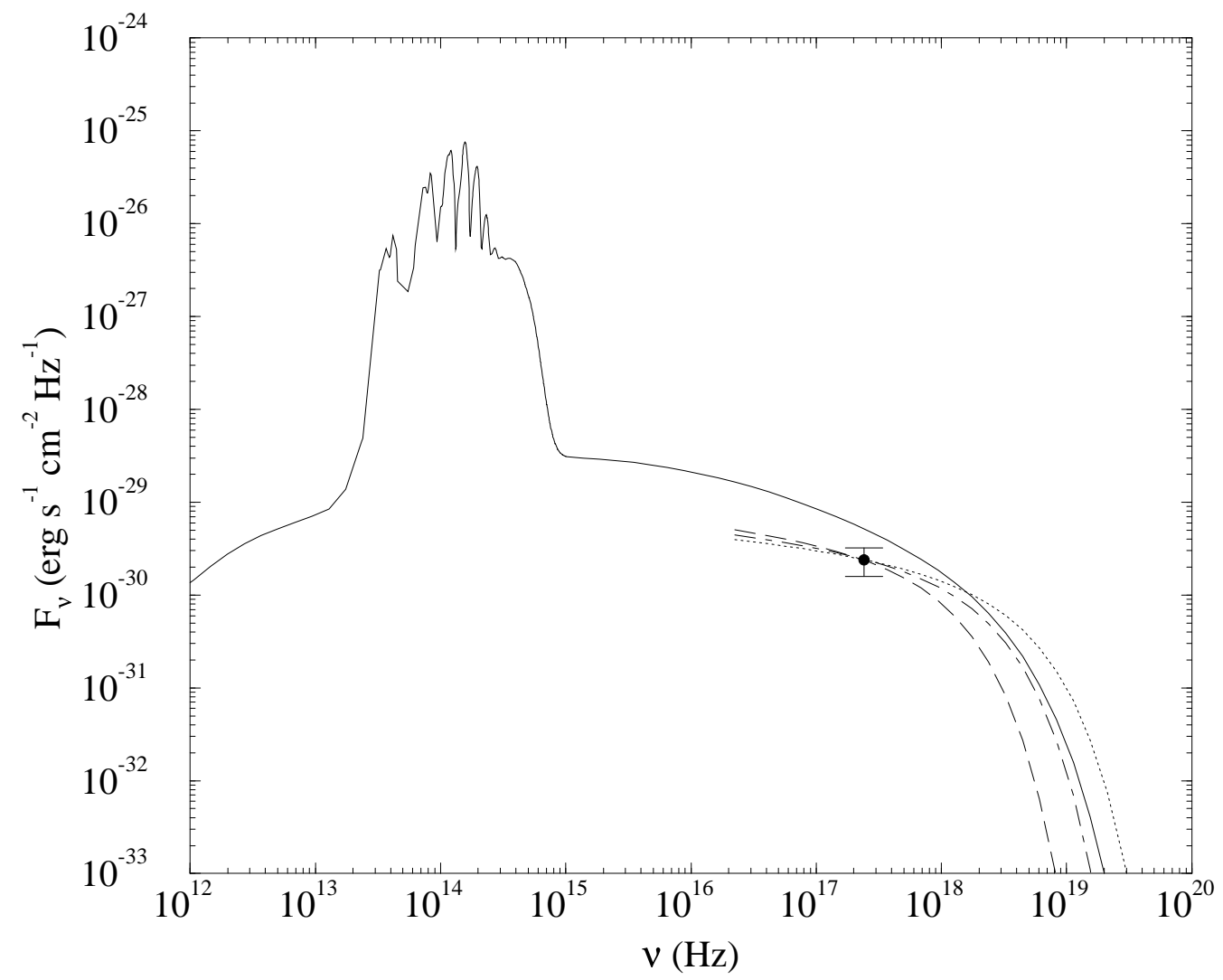

Abbildung 4.14: Gesamtspektrum der Anpassung im Infraroten und Röntgenbereich zu Bremstrahlung mit $k_{\mathrm{B}} T_{\mathrm{ff}}=10 \mathrm{keV}$. Dargestellt ist die Anpassung (durchgezogene Linie), sowie der Datenpunkt der ROSAT-Beobachtung vom September 1990 ( "low-state“) und isotherme Bremsstrahlungsspektren zu $k_{\mathrm{B}} T_{\mathrm{ff}}=5 \mathrm{keV}$ (langgestrichelte Linie), $10 \mathrm{keV}$ (strich-punktierte Linie), bzw. $20 \mathrm{keV}$ (gepunktete Linie), die als Bereich der spektralen Verläufe der Beobachtungen dienen.

\begin{tabular}{c|c|c}
$\dot{m}\left(\mathrm{~g} \mathrm{~cm}^{-2} \mathrm{~s}^{-1}\right)$ & $\Delta A_{\text {acc }}\left(\mathrm{cm}^{2}\right)$ & $\Delta \dot{M}\left(\mathrm{~g} \mathrm{~s}^{-1}\right)$ \\
\hline 100 & $1,2 \cdot 10^{11}$ & $1,2 \cdot 10^{13}$ \\
10 & $3,2 \cdot 10^{12}$ & $3,2 \cdot 10^{13}$ \\
1 & $2,4 \cdot 10^{13}$ & $2,4(1,2) \cdot 10^{13}$ \\
0,1 & $1,2 \cdot 10^{14}$ & $1,2(1,2) \cdot 10^{13}$ \\
$10^{-2}$ & $4,0 \cdot 10^{14}$ & $4,0\left(_{-4,0}^{+8,0}\right) \cdot 10^{12}$ \\
$10^{-3}$ & $2,8 \cdot 10^{16}$ & $2,8\left({ }_{-0,8}^{+0,4}\right) \cdot 10^{13}$ \\
$10^{-4}$ & $1,4 \cdot 10^{17}$ & $1,4\left(_{-0,8}^{+0,4}\right) \cdot 10^{13}$
\end{tabular}

Im Unterschied zur Anpassung des ,high-state" ist hier die Massenstromdichte $\dot{m}=$ $10^{-4} \mathrm{~g} \mathrm{~cm}^{-2} \mathrm{~s}^{-1}$ vertreten, da ohne ihren Beitrag die schmalen, hohen Zyklotronlinien bei $\lambda=1,9 \mu \mathrm{m}$ und $\lambda=2,4 \mu \mathrm{m}$ nicht wiedergegeben werden können. Wie auch bei der Anpassung des „high-state“, ist hier der Trend deutlich, daß die kleinen Massenstromdichten eine größere Fläche einnehmen als die hohen $\dot{m}$, damit ihre schwache Emission den beobachteten Zyklotronfluß mit schmalen Linien erzeugt. Die aus der Gewichtung 


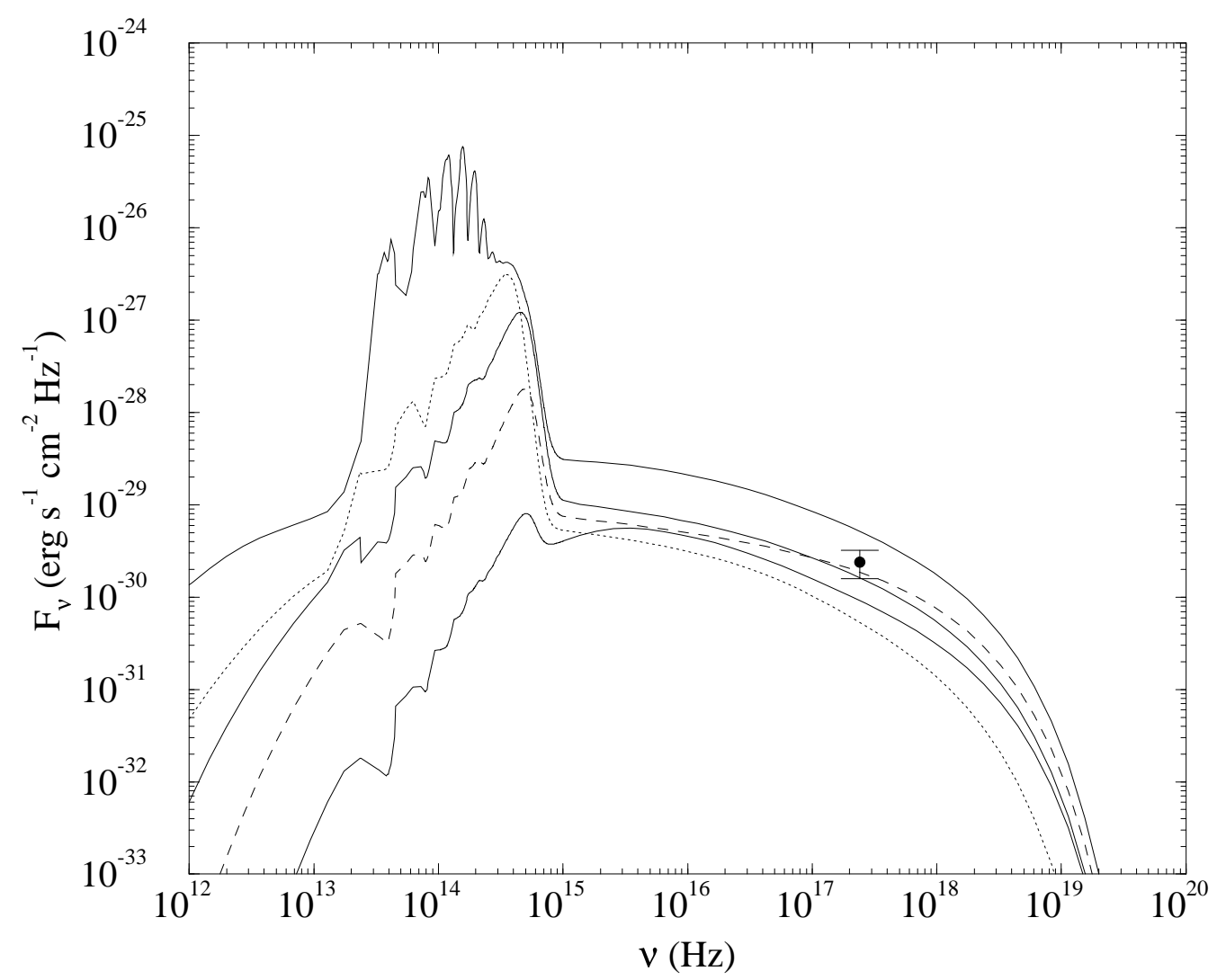

Abbildung 4.15: Anteile der einzelnen Massenstromdichten an der Anpassung in Abb. 4.14. Dargestellt ist die Anpassung (durchgezogene Linie) und die Teilspektren zu $\dot{m}=100$ (durchgezogene Linie), 10 (gestrichelte Linie), 1(durchgezogene Linie) und $10^{-1} \mathrm{~g} \mathrm{~cm}^{-2} \mathrm{~s}^{-1}$ (gepunktete Linie).

resultierende Akkretionsrate $\dot{M}=1,2 \cdot 10^{14} \mathrm{~g} \mathrm{~s}^{-1}$ ist zudem sehr viel kleiner als im Fall des „,high-state“, was die Vorstellung bestätigt, der „low-state“ sei ein Zustand, in dem das System nur geringe Akkretionstätigkeit aufweist.

In Abbildung 4.12 (S. 98) ist das gewichtete Zyklotronspektrum dargestellt und in Abbildung 4.13 (S. 99) die Anteile der Massenstromdichten $\dot{m}=10^{-4} \ldots 0,1 \mathrm{~g} \mathrm{~cm}^{-2} \mathrm{~s}^{-1}$ darin. Die Zyklotronlinien werden im wesentlichen durch die beiden Massenstromdichten $\dot{m}=10^{-4}$ und $10^{-3} \mathrm{~g} \mathrm{~cm}^{-2} \mathrm{~s}^{-1}$ gebildet. Die beiden folgenden $\dot{m}$ liefern im Infraroten nur Beitrag zum Kontinuum, und für $\dot{m}>1 \mathrm{~g} \mathrm{~cm}^{-2} \mathrm{~s}^{-1}$ ist der Anteil am Zyklotronspektrum vernachlässigbar. Die Gesamtanpassung des theoretischen Spektrums an die Beobachtung ist als recht gut anzusehen. Die noch bestehenden Differenzen um 2, $2 \mu \mathrm{m}$ kann man durch leicht veränderte Sichtwinkel beseitigen, die dann jedoch im „high-state" zu einer verschlechterten Anpassung führen. Auch sind gewisse Annahmen wie ein konstantes Magnetfeld über die Emissionsregion hinweg Vereinfachungen, die zu solchen Abweichungen führen können.

Der Gesamtverlauf der Gewichtung ist in Abbildung 1.14 gezeigt, und Abbildung 4.15 stellt die Anteile der Massenstromdichten dar, deren Hauptbeitrag im Röntgenbereich 


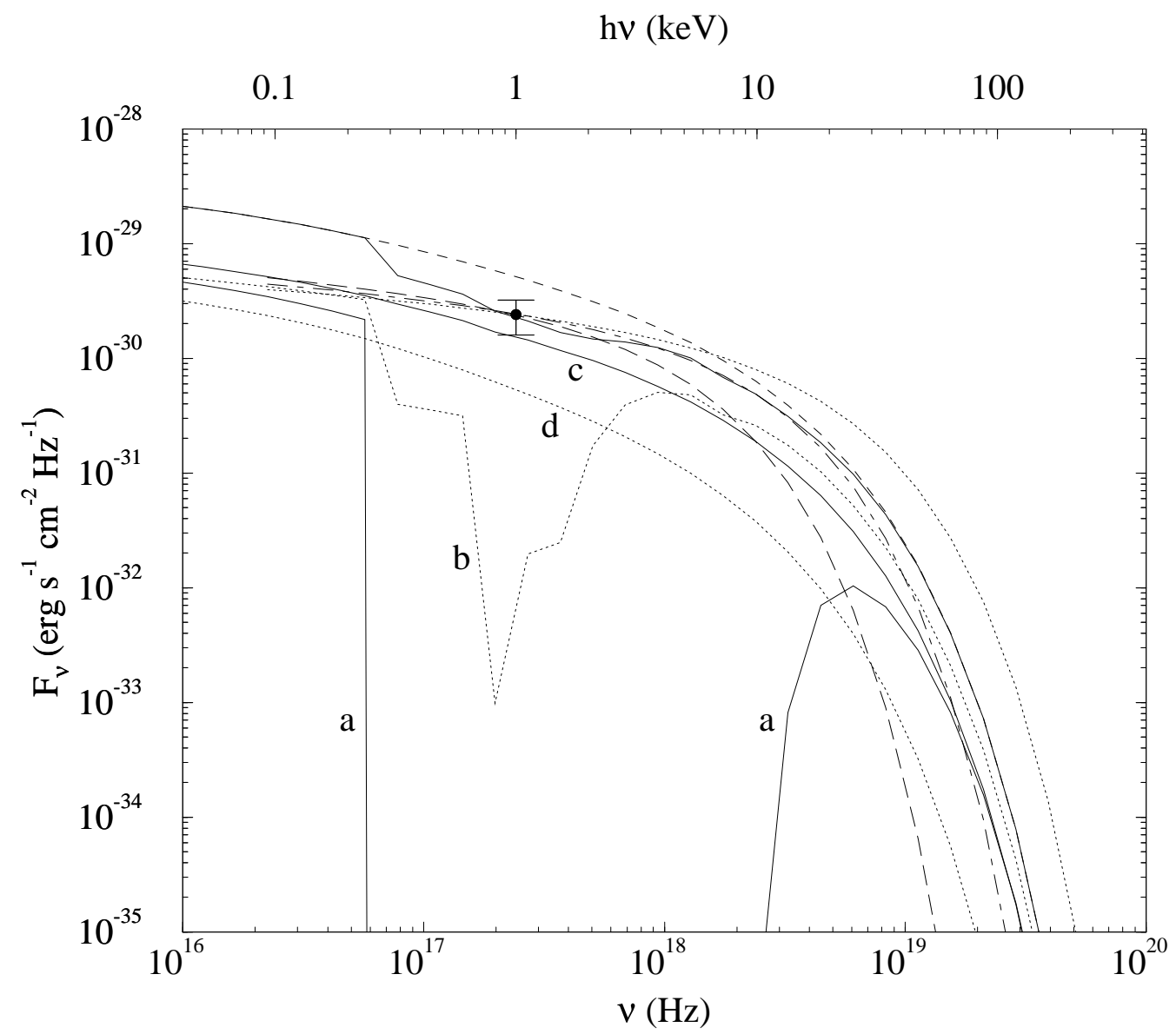

\begin{abstract}
Abbildung 4.16: Anpassung an den Bremsstrahlungsverlauf zu $k_{\mathrm{B}} T_{\mathrm{ff}}=10 \mathrm{keV}$ nach Absorption von Teilen der Röntgenstrahlung in der Atmosphäre des weißen Zwergs. Dargestellt ist die Anpassung (durchgezogene Linie) und der unabsorbierte Fluß (gestrichelte Linie), sowie die isothermen Bremsstrahlungsspektren zu $k_{\mathrm{B}} T_{\mathrm{ff}}=5 \mathrm{keV}$ (lang-gestrichelte Linie), $10 \mathrm{keV}$ (strich-punktierte Linie), bzw. $20 \mathrm{keV}$ (gepunktete Linie). Weiterhin die Anteile der Massenstromdichten (a) $\dot{m}=100$, (b) 10, (c) 1 und (d) $10^{-1} \mathrm{~g} \mathrm{~cm}^{-2} \mathrm{~s}^{-1}$.
\end{abstract}

liegt. Der Verlauf des harten Bereichs der Bremsstrahlungskurve wird durch die Anpassung hinreichend gut wiedergegeben, zumal die Wahl der Kurve zu $k_{\mathrm{B}} T_{\mathrm{ff}}=10 \mathrm{keV}$ nicht eine bestimmte Beobachtung wiedergeben sollte. Wichtig ist in diesem Zusammenhang nur der Befund, daß bei der Anpassung des harten Röntgenspektrums die hohen Massenstromdichten notwendig sind. Diese erzeugen aber einen Fluß bei $h \nu=1 \mathrm{keV}$, der über dem beobachteten ROSAT-Datenpunkt liegt. Die Verhältnisse sind also ganz ähnlich denen im "high-state“, und da das dort beschriebene Argument der Absorption von Röntgenstrahlung in der Umgebung der Stoßregion nicht an bestimmte Eigenschaften des „high-state“ geknüpft ist, kann es auch hier angewendet werden.

Um das berechnete Gesamtspektrum im Bereich $h \nu=1 \mathrm{keV}$ auf den durch den $R O$ SAT-Datenpunkt gegebenen Fluß zu bringen, wird die Strahlung der Massenstromdich- 


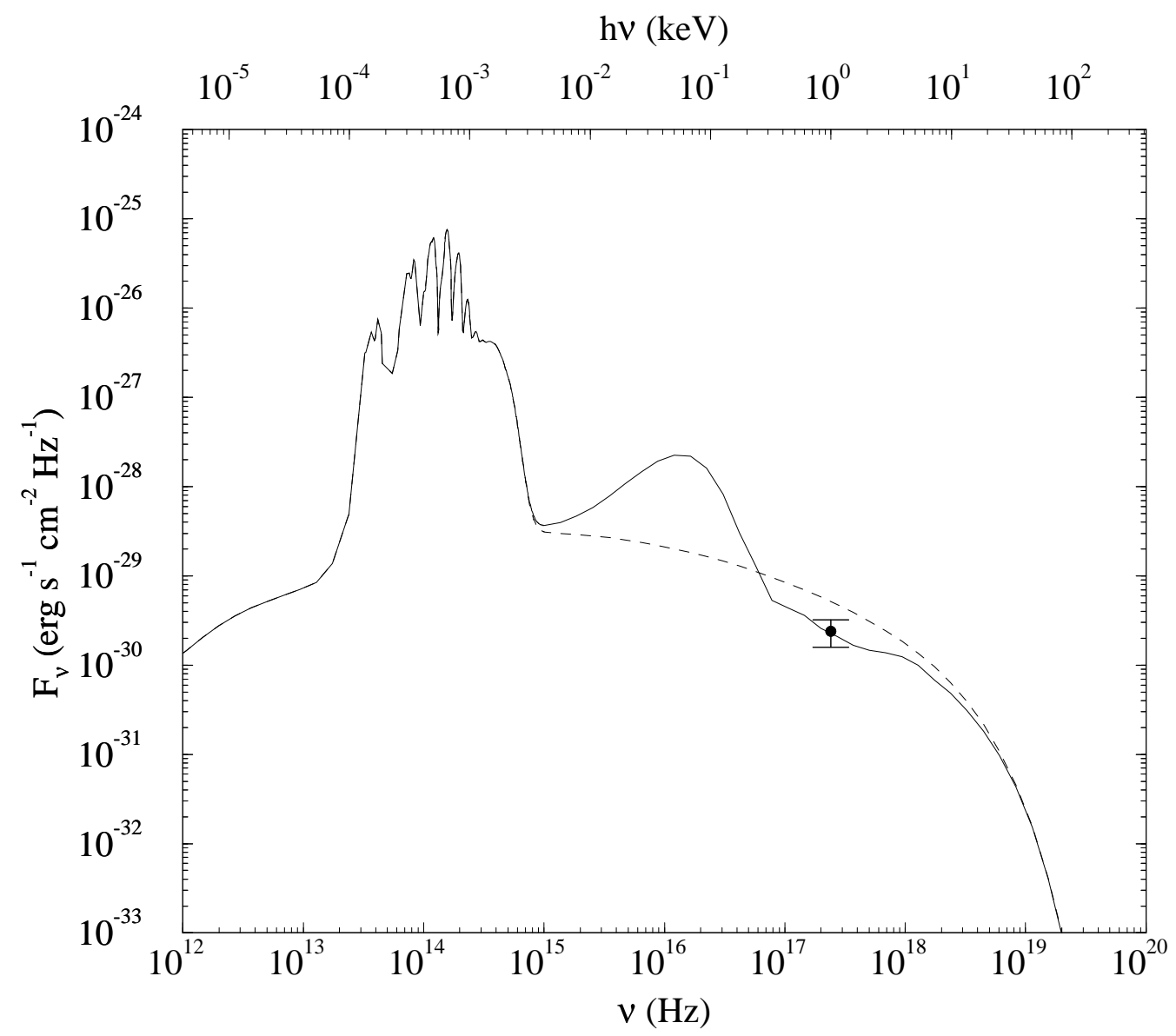

Abbildung 4.17: Schwarzkörperkomponente aus der bei der Absorption einbehalteten Strahlungsenergie. Dargstellt ist der unabsorbierte Fluß (gestrichelte Linie), sowie das durch Absorption abgeschwächte Spektrum mit der reprozessierten Schwarzkörperkomponente zu $k_{\mathrm{B}} T_{\mathrm{bb}} \approx 20 \mathrm{eV}$.

ten nach einer ähnlichen Abstufung in der Säulendichte $N_{\mathrm{H}}$ des umgebenden, absorbierenden Materials abgeschwächt, wie sie auch im Fall des „high-state" verwendet wurde. In Abbildung 4.16 sind die einzelnen Anteile der absorbierten Flüsse an der neuen Anpassung dargestellt. Die Strahlung aus Bereichen mit $\dot{m}=100 \mathrm{~g} \mathrm{~cm}^{-2} \mathrm{~s}^{-1}$ wird am stärksten absorbiert und liefert nur noch einen Beitrag zum Gesamtfluß für Frequenzen $\nu>3 \cdot 10^{19} \mathrm{~Hz}$. Das nächstkleinere $\dot{m}$ erzeugt nach der Abschwächung noch Strahlung, die zwischen $\nu \approx 10^{18} \mathrm{~Hz}$ und $\nu \approx 10^{19} \mathrm{~Hz}$ dominiert. Dagegen wird die Emission aus Gebieten mit $\dot{m}=1 \mathrm{~g} \mathrm{~cm}^{-2} \mathrm{~s}^{-1}$ nur sehr gering abgeschwächt. Daher wird der Fluß der ROSAT-Beobachtung bei $h \nu=1 \mathrm{keV}$ im wesentlichen durch diese Massenstromdichte geliefert. Die kleineren Massenstromdichten sind unabsorbiert und tragen nur unerheblich zum Röntgenfluß bei.

Die bei der Absorption einbehaltene Energie wird in Form von Schwarzkörperstrahlung wieder emittiert und erzeugt einen weichen Röntgenanteil, der zusätzlich zu den Komponenten der Zyklotron- und Bremsstrahlung im Gesamtspektrum auftreten muß. 


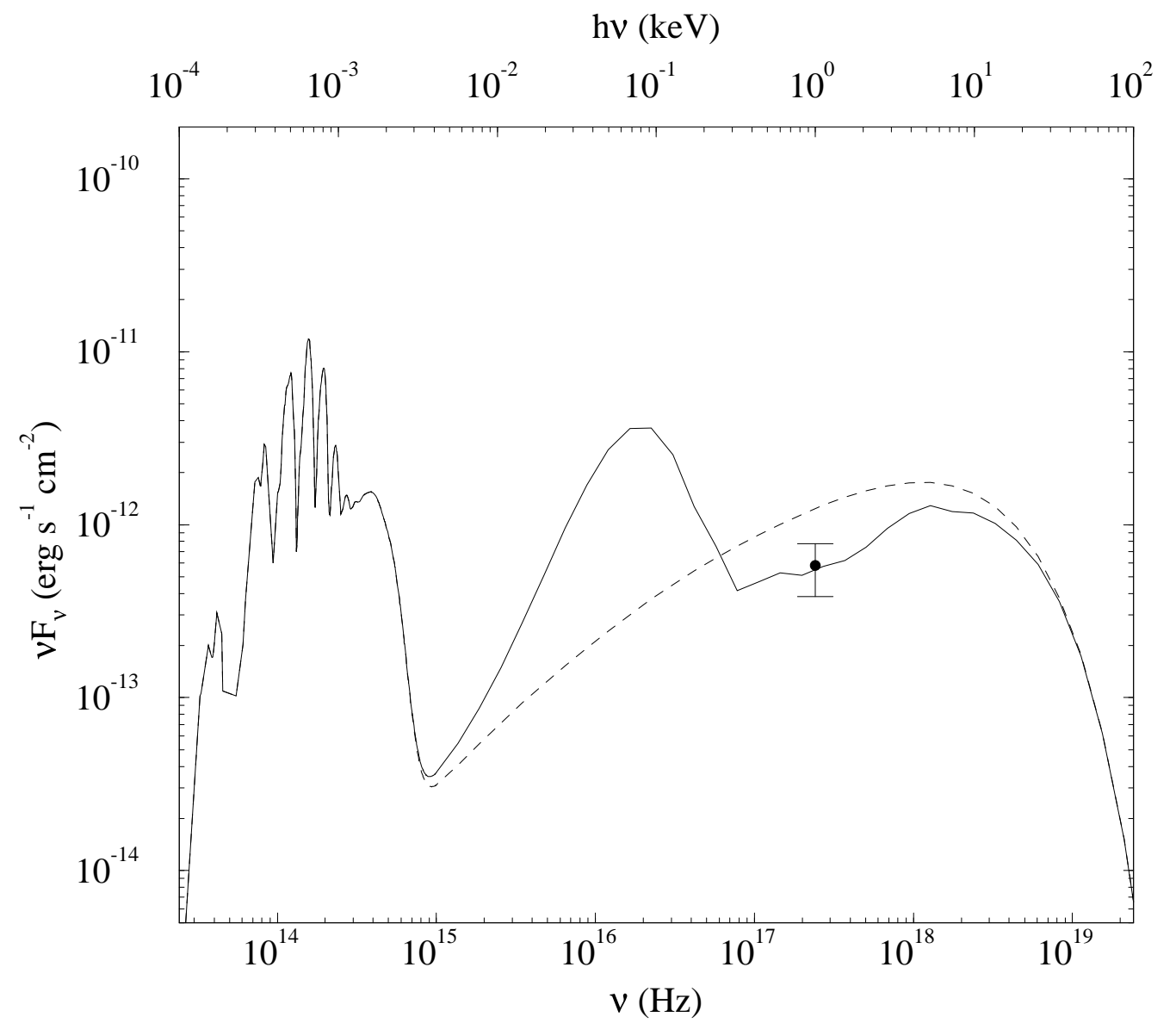

Abbildung 4.18: Darstellung des „low-state“-Energieinhalts der einzelnen Komponenten aus Abb. 4.17. Nicht dargestellt sind die Beiträge aus der Emission des weißen Zwergs, des Sekundärsterns und des Akkretionsstroms.

Die Differenz der Flüsse aus unabsorbiertem Gesamtspektrum und Flußverlauf nach der Abschwächung der Beiträge der einzelnen Massenstromdichten ergibt für die oben beschriebene Anpassung einen absorbierten Fluß von $f_{\text {abs }}=2,3 \cdot 10^{-12} \mathrm{erg} \mathrm{s}^{-1} \mathrm{~cm}^{-2}$. Verknüpft man diese Strahlung mit dem Doppelten der Summe der Flächenanteile zu den Massenstromdichten $\dot{m}=100$ bzw. $10 \mathrm{~g} \mathrm{~cm}^{-2} \mathrm{~s}^{-1}$, so ergibt sich eine Schwarzkörperstrahlung zu der Temperatur $k_{\mathrm{B}} T_{\mathrm{bb}} \approx 20 \mathrm{eV}$ mit einem Fluß $f_{\mathrm{bb}}=4,6 \cdot 10^{-12} \mathrm{erg} \mathrm{s}^{-1} \mathrm{~cm}^{-2}$, die in Abbildung 4.17 (vorherige Seite) zusammen mit dem unabsorbiertem Spektrum dargestellt ist. Um eine Einschätzung über den Energieinhalt der Komponenten zu bekommen, ist in Abbildung 4.18 die Darstellung $\nu F_{\nu}$ gegen $\nu$ gewählt. Der Anteil der Schwarzkörperkomponente an der Strahlungsenergie ist hier gering, und die anderen Komponenten dominieren die Energieverteilung. Im „low-state" sollte also kein drastischer Überschuß an weicher Röntgenstrahlung zu erwarten sein.

In diesem Kapitel konnte somit gezeigt werden, daß eine Anpassung des Gesamtspektrums des „low-state“ von AM Her ebenfalls eine Verteilung von Massenstromdichten erfordert, damit sowohl der Fluß im Infraroten als auch die Röntgenstrahlung schlüssig 
beschrieben werden. Weiterhin konnte gezeigt werden, daß mit dem plausiblen Argument der Absorption von Teilen der Röntgenstrahlung einer Akkretionssäule in der Atmosphäre des weißen Zwergs, auch im Zustand geringer Akkretionstätigkeit eine - wenn auch schwache - Schwarzkörperkomponente zu erwarten ist. Diese Komponente sollte aber wesentlich geringer sein als bei einem „high-state“, so daß hier der Überschuß an weicher Röntgenstrahlung weit geringer ausfällt.

\subsubsection{Diskussion}

In den beiden vorangehenden Kapiteln wurde gezeigt, daß das beobachtete Gesamtspektrum des Polars AM Her im ",high-state" wie auch im „low-state" durch Strahlung aus Gebieten der Akkretionsregion erklärt werden kann, deren Massenstromdichten über einen weiten Bereich variieren. Die jeweilige Anpassung ergab dabei, daß die Fläche der Regionen mit kleinen Massenstromdichten erheblich größer ist, als die Ausdehnung der Gebiete mit hohem $\dot{m}$. Die bei der durchgeführten Gewichtung gefundene Fläche zu einer festen Massenstromdichte ist für die beiden betrachten Zustände des Polars aber nicht identisch. Der Beitrag der einzelnen Massenstromdichten zur gesamten Akkretionsrate eines Zustands wird damit ebenfalls unterschiedlich sein.

In Abbildung 4.19 (folgende Seite) ist der differentielle Beitrag $\Delta \dot{M}$ einer Massenstromdichte in der Anpassung des Gesamtspektrums des "high-state" und des "low-state“ dargestellt; die Akkretionsrate $\dot{M}$ ergibt sich durch Summation über die differentiellen Beiträge $\Delta \dot{M}$. Die Fehlergrenzen der Datenpunkte für $\dot{m} \leq 1 \mathrm{~g} \mathrm{~cm}^{-2} \mathrm{~s}^{-1}$ resultieren aus der Qualität der für die Anpassung verwendeten Beobachtung; eine untere Fehlergrenze außerhalb der Darstellung ist äquivalent mit dem möglichen Fehlen dieser Massenstromdichte in der Anpassung. Der globale Verlauf der Verteilung der Massenstromdichten ist für die beiden Zustände stark unterschiedlich: während die Verteilung für den „,high-state“ zu hohen Massenstromdichten hin ansteigt, zeigt der "low-state" eher ein flacher Verlauf.

In beiden Zuständen sind Massenstromdichten $\dot{m} \leq 10^{-1} \mathrm{~g} \mathrm{~cm}^{-2} \mathrm{~s}^{-1}$ notwendig. Im "low-state "ist ein unterer Wert von $\dot{m}=10^{-4} \mathrm{~g} \mathrm{~cm}^{-2} \mathrm{~s}^{-1}$ benutzt worden, um die schmalen Zyklotronlinien der Beobachtung zu erzeugen. Obwohl es sich beim „high-state“-,Spektrum" nur um Breitbandphotometrie handelt, die weniger genau ist, muß auch hier eine kleine Massenstromdichte eingeführt werden, um den Verlauf der Emission für $\lambda \approx 1,6 \mu \mathrm{m}$ zu reproduzieren. Da der Akkretionsstrom vom Begleiter des weißen Zwergs gespeist wird, kann eine mögliche Erklärung für das Vorhandensein der kleinen Massenstromdichten zu beiden Akkretionszuständen durch dynamische Prozesse der Sekundärsternatmosphäre gegeben werden. Der Materieüberstrom geschieht in der Nähe des $L_{1}$-Punkts. Wenn nun die Atmosphäre des Begleiters über diesen Punkt ausgedehnt ist (z. B. durch eine Heizung auf Grund der Bestrahlung durch den weißen Zwerg), werden dichte Bereiche der Photosphäre zum Materiestrom beitragen; die Massenstromdichten sind hoch und der Polar geht in einen „high-state“. Da wegen der thermischen Verteilung der Teilchen des Gases auch weniger dichte Gebiete der äußeren Sternatmosphäre den Potentialwall bei $L_{1}$ passieren können, sind in diesem Zustand auch noch geringe Massenstromdichten vertreten. Zieht sich die Photosphäre des Begleiters jedoch lokal zurück, indem sich z. B. ein Stern- 


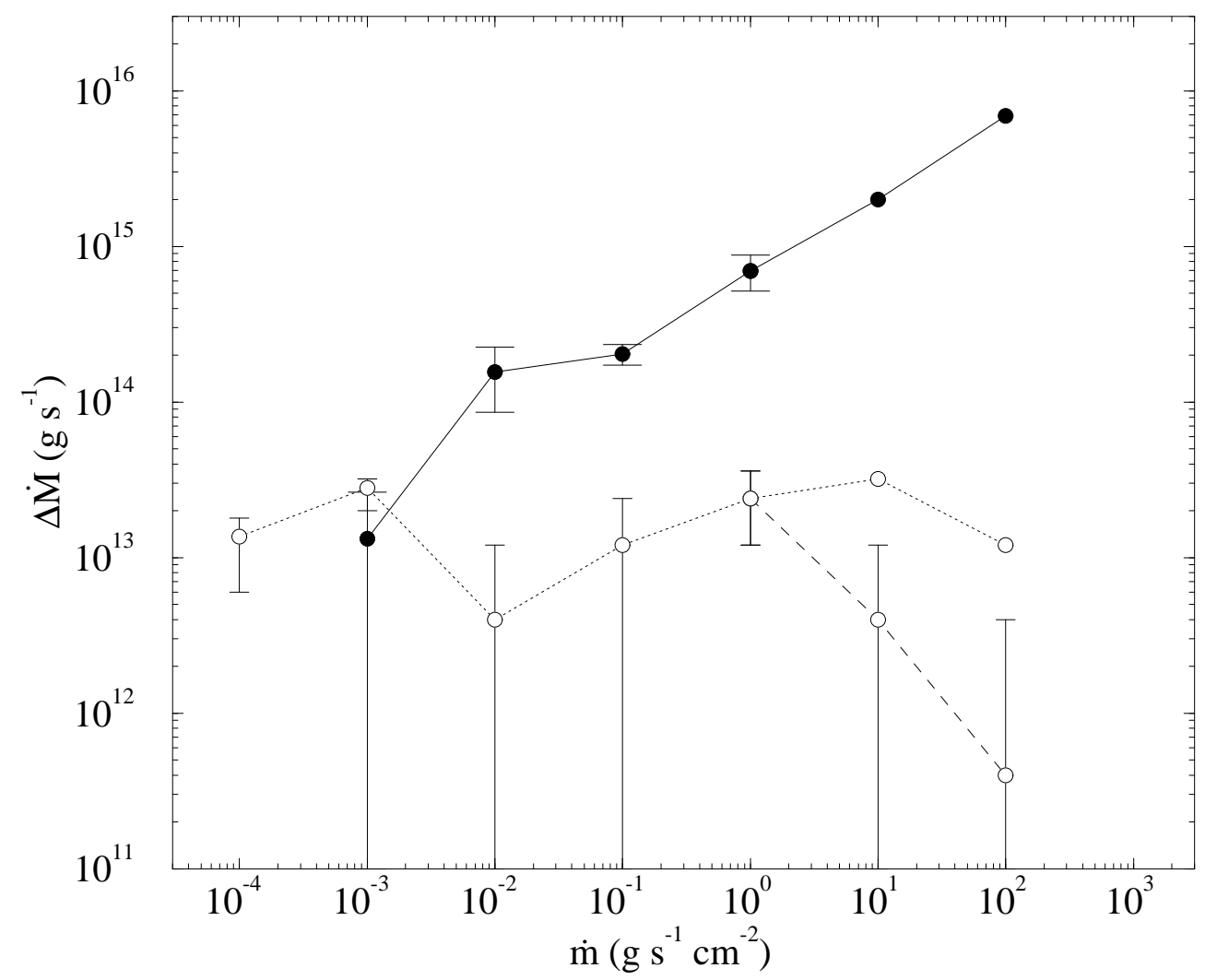

Abbildung 4.19: Anteile der Massenstromdichten $\dot{m}$ an der Akkretionsrate $\dot{M}$. Dargestellt ist die differentielle Akkretionsrate $\Delta \dot{M}:=\dot{m} \Delta A_{\text {acc }}$ für den „high-state“ (Kreise; siehe Tabelle in Kap. 4.2.1) und "low-state" (offene Kreise) mit beobachtbarer Schwarzkörperstrahlung (gepunktete Linie; siehe Tabelle in Kap. 4.2.2) bzw. nicht nachgewiesener Reprozessionsstrahlung (gestrichelte Linie).

fleck mit geringer Temperatur vor den $L_{1}$-Punkt schiebt, werden nur die Gebiete geringer Dichte (Korona, Chromosphäre) den $L_{1}$-Punkt erreichen, und die Akkretionsrate sinkt ab, so daß der Anteil der hohen Massenstromdichten abfällt; der Polar befindet sich nun in einem "low-state".

Daß im „low-state" der Anteil der Massenstromdichten $\dot{m} \geq 1 \mathrm{~g} \mathrm{~cm}^{-2} \mathrm{~s}^{-1}$ nicht null ist, wird durch das auch in diesem Zustand recht harte Röntgenspektrum erzwungen. Tatsächlich läßt sich eine Anpassung an den infraroten Anteil des Gesamtspektrums durchführen, die zwar auch noch den ROSAT-Datenpunkt wiedergibt, jedoch für Energien $1<h \nu<10 \mathrm{keV}$ zu rasch an Fluß verliert und unterhalb der Bremsstrahlungskurve zu $k_{\mathrm{B}} T_{\mathrm{ff}}=10 \mathrm{keV}$ bleibt, was dem spektralen Verlauf der ROSAT-Beobachtungen widerspricht. Deren Verteilung der Massenstromdichten ist in Abbildung 4.19 als gestrichelte Kurve dargestellt. Die Anteile der Massenstromdichten zu $\dot{m}=10$ und $100 \mathrm{~g} \mathrm{~cm}^{-2} \mathrm{~s}^{-1}$ sind mit Null verträglich; bei einer Gewichtung mit dem Wert der oberen Fehlergrenze für $\Delta \dot{M}$ würde das resultierende Röntgenspektrum den oberen Fehlerbereich des ROSATDatenpunkts erreichen. So ist die Vorstellung, daß einem „low-state" ein Zustand geringer 
Akkretionstätigkeit entspricht zwar richtig, da die Akkretionsrate $\dot{M}$ in der Tat geringer ist als im "high-state" $\left(\dot{M}_{\text {low }}=1,2 \cdot 10^{14} \mathrm{~g} \mathrm{~s}^{-1}, \dot{M}_{\text {high }}=1,0 \cdot 10^{16} \mathrm{~g} \mathrm{~s}^{-1}\right)$, aber offenbar sind weiterhin lokale Erhöhungen der Massenstromdichten anzutreffen.

Aus der Beobachtung von harten Röntgenspektren im „high-state“ und einem ähnlich harten Flußverlauf im „low-state" folgt die Anwesenheit von hohen Massenstromdichten zu beiden Zuständen. Eine plausible Betrachtung der Druckverhältnisse, die bei einer Akkretion von hohen Massenstromdichten in der Atmosphäre des weißen Zwergs herrschen, läßt es unausweichlich erscheinen, daß wenigstens die hohen Werte einer Verteilung von Massenstromdichten vollständig in die Sternatmosphäre einsinken. Die von diesen Teilen der Verteilung abgestrahlte Energie wird daher unter der Photosphäre deponiert und erreicht nur als reprozessierte Strahlung den Beobachter. An Hand dieser Vorstellung können das harte Röntgenspektrum beider Akkretionszustände sowie die intensive weiche Röntgenstrahlung des Polars AM Her im „high-state“ quantitativ wiedergegeben werden. Für den "low-state" konnte wenigstens qualitativ dargelegt werden, daß aus einem harten Röntgenspektrum das Vorhandensein einer schwachen, schwarzkörperartigen weichen Röntgenkomponente folgen muß.

Die zur Anpassung eines vorgegebenen Bremsstrahlungsverlaufs im harten Röntgenbereich benötigten Anteile der Massenstromdichten $\dot{m}=10$ und $100 \mathrm{~g} \mathrm{~cm}^{-2} \mathrm{~s}^{-1}$ sind in Abbildung 4.19 eingetragen. Für diese Datenpunkte können keine zuverlässigen Fehlergrenzen angegeben werden, da keine direkte Anpassung an reale Beobachtungsdaten der harten Röntgenstrahlung durchgeführt, sondern nur ein qualitativer Verlauf verwendet wurde. Der Variationsbereich dieser $\dot{m}$ in der Anpassung des „high-state“ ist jedoch nicht sehr groß, denn sowohl die resultierende Akkretionsrate $\dot{M}$ als auch der aus der Absorption von Teilen der harten Bremsstrahlung stammende Schwarzkörperfluß $f_{\mathrm{bb}}$ der weichen Röntgenstrahlung stimmen mit aus Beobachtungen abgeleiteten Werten gut überein. Es ist aber durchaus möglich, die gleichen Werte für $\dot{M}$ und $f_{\mathrm{bb}}$ mit einer Erhöhung des Anteils von $\dot{m}=10 \mathrm{~g} \mathrm{~cm}^{-2} \mathrm{~s}^{-1}$ und einer Herabsetzung des von $\dot{m}=100 \mathrm{~g} \mathrm{~cm}^{-2} \mathrm{~s}^{-1} \mathrm{zu}$ erreichen. Solch ein Argument gilt im Übrigen auch für die Hinzunahme eine Massenstromdichte von $\dot{m}=1000 \mathrm{~g} \mathrm{~cm}^{-2} \mathrm{~s}^{-1}$, die eine Herabsetzung des Anteils von $\dot{m}=100 \mathrm{~g} \mathrm{~cm}^{-2} \mathrm{~s}^{-1}$ erfordern würde. Allerdings wäre der Anteil dieses hohen $\dot{m}$ gering, da sonst ein zu hoher harter Röntgenfluß vorhergesagt würde. Im Fall des „low-state“ sind weder harte Röntgenstrahlung noch weiche Schwarzkörperstrahlung hinreichend gut vorgegeben, um Fehlerbereiche für die Anteile der hohen Massenstromdichten anzugeben. Man kann jedoch feststellen: Wenn eine schwarzkörperartige Komponente in einem beobachteten Spektrum zu finden ist, dann sind die Anteile der $\dot{m}=10$ und $100 \mathrm{~g} \mathrm{~cm}^{-2} \mathrm{~s}^{-1}$ nicht kleiner als die oberen Fehlergrenzen der entsprechenden Punkte zu der gestrichelten Kurve in Abbildung 4.19, denn diese Anteile der Massenstromdichten erzeugen einen Bremsstrahlungsverlauf, der den ROSAT-Datenpunkt nicht überschreitet, somit keine nachweisbare reprozessierte Strahlung liefert. Allerdings ist das resultierende harte Röntgenspektrum dann u. U. nicht hart genug, um konsistent mit der Beobachtung zu sein.

Um die im vorherigen ausgeführten Ergebnisse zu festigen, ist als nächster Schritt eine Anpassung an reale Röntgendaten durchzuführen. Dazu ist allerdings die Berücksichtigung der bisher vernachlässigten Streuung der Röntgenstrahlung aus der Akkretionsregi- 
on an der Oberfläche des weißen Zwergs nötig. Theoretische Rechnungen zeigen, daß eine Bestrahlung der Sternoberfläche mit harter Bremsstrahlung wegen Compton-Streuung an freien Elektronen der Atmosphäre eine Albedo ergibt, die das Spektrum im Bereich $10 \mathrm{keV}<h \nu<50 \mathrm{keV}$ verstärkt (van Teeseling, Kaastra \& Heise 1996). Die grundsätzlichen hier getroffenen Aussagen über die Strukturierung der Akkretionsregion werden durch diese Korrektur nicht berührt.

Bezüglich der Energiebilanz der Akkretion unterscheidet sich das hier vorgestellte Modell wesentlich von dem über 20 Jahre hinweg diskutierten Modell von Lamb \& Masters (1979). Deren Modell sagte eine Energiebilanz der Form $L_{\mathrm{bb}}=L_{\mathrm{cyc}}+L_{\mathrm{ff}}$ voraus. Die Basis dieser Bilanz besteht darin, daß die Hälfte der emittierten Leistung in Form von Zyklotronund Bremsstrahlung die Sternoberfläche treffen und dann als weiche Röntgenstrahlung der geheizten Oberfläche wieder erscheinen sollte. Die Tatsache, daß die beobachtete weiche Röntgenstrahlung um einen Faktor bis zu 100 intensiver ist als von Lamb \& Masters vorausgesagt, wurde als das sog. „Soft X-Ray Puzzle“ bekannt. Gänsicke, Beuermann \& de Martino (1995) konnten jedoch zeigen, daß die Zyklotron- und die Bremsstrahlung der Akkretionssäule die Atmosphäre in einem weiteren Umkreis des Akkretionsflecks aufheizen. Damit kann die UV-Strahlung des weißen Zwergs, und nicht die wie von Lamb \& Masters behauptete Schwarzkörperstrahlung, als reprozessierte Komponente der direkten Strahlung der Akkretionssäule erklärt werden. Das hier vorgestellte Modell berücksichtigt dagegen den entscheidenden Anteil, den die Photoabsorption der Bremsstrahlung an der Erzeugung der weichen Röntgenstrahlung hat und erklärt diese Komponente als natürliche Auswirkung der Akkretion hoher Massenstromdichten. Das "Soft X-Ray Puzzle" ist somit keine rätselhafte, sondern eine normale Erscheinung der Akkretionsprozesse.

Das in den obigen Kapiteln aufgestellte Modell mit einer Strukturierung der Akkretionsregion in einzelne Massenstromdichten ist ein Teilaspekt des allgemeineren Problems der instationären Akkretion mit einer kontinuierlichen räumlichen Verteilung der Massenstromdichten. Während hier Aussagen über das zeitliche Mittel der Strahlung einer in Klassen $\dot{m}_{i}<\dot{m}<\dot{m}_{i+1}$ eingeteilten Akkretionsregion gemacht werden, muß im allgemeinen Zugang eine zeitliche Entwicklung der Stöße (mit eventuell resultierenden Oszillationen der Emissionsregion) gewährleistet werden, wobei u. U. sogar eine zeitabhängige Akkretionsrate $\dot{M}(t)$ berücksichtigt werden sollte. Darüber hinaus ist die Modellierung einer zweidimensionalen, kontinuierlichen Verteilung der Massenstromdichten über den Akkretionsfleck hinweg notwendig. Zur korrekten Einbeziehung der Strahlungsprozesse in der Emissionsregion, ist des weiteren der zeitabhängige dreidimensionale Strahlungstransport zu lösen. Das komplette System der dazu notwendigen Differentialgleichungen ist aber höchstwahrscheinlich auch unter größtem numerischen Aufwand nicht zu lösen. Daher kann eine Erweiterung des vorgestellten Modells nur in einer Betrachtung eines weiteren Teilaspekts bestehen. Lediglich bezüglich einer Zeitabhängigkeit der Massenstromdichte dürfte sich das hier dargestellte Modell nicht von einer vollständig zeitabhängig durchgeführten Rechnung unterscheiden, wenn man nur Zeitmittel der resultierenden Spektren betrachtet. So ist es z. B. ohne Belang, ob eine Emissionsregion oszilliert oder stationär ist, wenn sie auf Grund ihrer hohen Massenstromdichte vollständig in der Atmosphäre liegt. 


\section{Kapitel 5}

\section{Zusammenfassung und Ausblick}

Das Phänomen der gravitativen Massenansammlung („Akkretion“) auf ein stellares Objekt ist im Universum weit verbreitet und tritt in verschiedensten Ausprägungen auf. In dieser Arbeit wird nun der Akkretionsvorgang in Doppelsternsystemen, bestehend aus magnetischem weißen Zwerg und sonnenähnlichem Stern, untersucht. Diese Systeme werden wegen ihrer Strahlungseigenschaften auch „Polare“ genannt. Da die sonnenähnliche Komponente einen Teil des resultierenden Gravitationspotentials des Systems vollständig ausfüllt, fließt Materie (i. allg. ein Plasma) über einen Sattelpunkt des Potentials auf den weißen Zwerg, der auch die Komponente mit der größeren Masse ist. Der weiße Zwerg besitzt ein so hohes Magnetfeld, daß der Materiestrom entlang der Feldlinien auf die Sternoberfläche geführt wird. Beim Fall im Gravitationspotential des Sterns erreicht der Strom Überschallgeschwindigkeit, so daß die Abbremsung auf thermische Geschwindigkeiten auf der Oberfläche des weißen Zwergs in Form eines starken hydrodynamischen Stoßes geschieht, in dem die Materie auf hohe Temperaturen geheizt wird. Durch Strahlungsprozesse verliert das Plasma einen Teil der beim Fall gewonnenen Energie wieder, und zwischen der Stoßfront und der Sternoberfläche wird sich eine Temperatur- und Dichteverteilung ausbilden, deren Ausdehnung durch die Effizienz der Verluste bestimmt wird. Die Strahlung dieser strukturierten Region läßt nun Rückschlüsse auf die Systemparameter wie z. B. Magnetfeldstärke oder Masse des weißen Zwergs zu.

Die beobachtete starke Strukturierung der Massenstromdichte in der Akkretionsregion zeigt, daß der Akkretionsstrom in starkem Maße inhomogen ist. Diese Inhomogenitäten entstehen wahrscheinlich schon im $L_{1}$-Punkt am Sekundärstern, können aber durch Turbulenz und Plasmainstabilitäten bei der Abbremsung des Stroms in der Magnetosphäre des weißen Zwergs verstärkt werden. Über viele dieser zeitlichen und räumlichen Inhomogenitäten muß man beim derzeitigen Stand der Kenntnis hinwegmitteln.

Ein einfaches Modell der Akkretionsprozesse wurde aufgestellt, indem der Plasmastrom als eindimensionales, stationäres, hydrodynamisches Fluid bestehend aus Elektronen und Protonen angenommen wird. Dafür wurden Kontinuitäts-, Navier-Stokes- und Energiegleichung mit den Rankine-Hugoniot-Randbedingungen für einen starken Stoß gelöst. Die Navier-Stokes-Gleichung wurde mit und ohne viskose Terme untersucht. Es zeigte sich, daß die Viskosität nur für die Erzeugung und den Ort des Stoßes wichtig ist, 
sonst aber keinen signifikanten Einfluß auf die physikalischen Größen der Schicht hat. Um die numerischen Probleme bei der Lösung der Differentialgleichungen zu verringern, wurden daher die viskosen Terme vernachlässigt und die Ausbildung eines starken Stoßes über dem weißen Zwerg vorausgesetzt. Weiterhin konnte begründet dargestellt werden, daß Wärmeleitung der Elektronen und Effekte durch Compton-Streuung im Parameterbereich der Temperatur und Dichte in Polaren keinen wesentlichen Beitrag zur Struktur der Emissionsregion liefert; diese Prozesse wurden daher vernachlässigt. Die Energiegleichung wurde für Elektronen und Protonen getrennt aufgestellt, die Coulomb-Wechselwirkung der Teilchen wurde durch einen Kopplungsterm in beiden Gleichungen berücksichtigt. Weiter wurde angenommen, daß nur die Elektronen Strahlungsverluste durch Zyklotronund Bremsstrahlung aufweisen. Zyklotronstrahlung verläßt im Gegensatz zur Bremsstrahlung das Plasma nicht vollständig, sondern trägt zum Teil zur Aufheizung bei („optisch dicker Strahlungstransport"). Es ist daher nicht möglich, Zyklotronstrahlung als einfachen Verlustterm in die Energiegleichung zu integrieren. Stattdessen muß eine zusätzliche Differentialgleichung („Strahlungstransportgleichung“) gelöst werden, die den Energietransport durch Strahlung in der Emissionsregion beschreibt. Dies bedeutet einen erheblichen numerischen Mehraufwand, da sich die hydrodynamischen Gleichungen und der Strahlungstransport nicht zu einem geschlossenen System verbinden lassen. Zur Lösung des Problems wurde ein iteratives Verfahren gewählt, um Energieerhaltung sicherzustellen.

Mit diesem Modell wurden Geschwindigkeits-, Dichte- und Temperaturverteilungen in Abhängigkeit von Massenstromdichte $\left(\mathrm{g} \mathrm{cm}^{-2} \mathrm{~s}^{-1}\right)$, Masse und Magnetfeld des weißen Zwergs berechnet. Das iterative Verfahren erwies sich dabei über einen weiten Bereich dieser stellaren Parameter als stabil. Die Geschwindigkeit des Plasmastroms wird vom Wert für den freien Fall vor dem Stoß auf einen kleineren Wert dahinter gebracht und nimmt dann stetig bis auf thermische Geschwindigkeiten an der Oberfläche des Sterns ab. Die Kontinuitätsgleichung legt den Dichteverlauf fest und bewirkt einen starken Anstieg in der Nähe des weißen Zwergs. Der Verlauf der Elektronentemperatur wird durch die Coulomb-Wechselwirkung mit den Protonen und durch Strahlungsverluste bestimmt. Wegen ihrer größeren Masse bringen die Protonen die durch den Fall im Gravitationsfeld des weißen Zwergs gewonnene Energie in die Schicht ein. Diese Energie wird durch Dissipation im Stoß in Wärme umgewandelt, so daß die Protonen instantan (genauer: innerhalb einer freien Weglänge) auf die Rankine-Hugoniot-Temperatur geheizt werden. Die Elektronen werden von einer wesentlich kleineren Anfangstemperatur durch CoulombWechselwirkung mit den heißen Protonen aufgeheizt. Gleichzeitig kühlen die Elektronen durch Strahlungsverluste. Die Zyklotronstrahlung ist dabei effektiv in Bereichen mit hoher Temperatur, während die Bremsstrahlung bei hoher Dichte dominant ist. Diese Prozesse sorgen somit für einen Anstieg der Elektronentemperatur bis auf einen Maximalwert und eine Temperaturabnahme bis auf einen Endwert, der größer ist als die Temperatur eines weißen Zwergs ohne Akkretion. Die Maximaltemperatur der Elektronen und die geometrische Höhe der geheizten Region werden bei den betrachteten Verlustprozessen durch die stellaren Parameter festgelegt. Dabei bewirkt bei konstantem Magnetfeld eine geringe (hohe) Massenstromdichte eine niedrige (hohe) Maximaltemperatur und weite (kurze) Ausdehnung der Schicht, während bei konstanter Massenstromdichte ein kleines (großes) 
Magnetfeld eine hohe (geringe) Maximaltemperatur und eine weit (kurz) ausgedehnte Schicht zur Folge hat.

Die mit diesen Schichtungen berechnete freigesetzte Strahlung der geheizten Region zeigt für die gewählten stellaren Parameter charakteristische Merkmale. Damit lassen sich durch einen Vergleich mit Beobachtungen (Spektralbereich von Infrarot bis Röntgenstrahlung) Aussagen über die Plasmaeigenschaften der Emissionsregion konkreter Systeme machen, um so auf stellare Größen wie Magnetfeld, Masse des weißen Zwergs und Massenstromdichte auf der Sternoberfläche zu schließen. In der Literatur wird dafür häufig eine Näherungslösung für die Temperaturverteilung der Plasmasäule verwendet, die einen einfachen Ansatz für die Strahlungskühlung macht und keine Unterscheidung zwischen Elektronen- und Protonentemperatur vorsieht („Ein-Teilchen-Beschreibung“"). Daher wurde zunächst untersucht wie stark die Abweichungen zu den Schichtungen sind, die mit obigem Ansatz („Zwei-Teilchen-Beschreibung“) berechnet wurden. In der Ein-TeilchenBeschreibung wird angenommen, daß die Elektronen ohne langsamen Temperaturanstieg instantan im Stoß auf die Rankine-Hugoniot-Temperatur geheizt werden und von diesem Wert herunterkühlen, wobei die Kühlung durch einen reinen Verlustterm in den Differentialgleichungen beschrieben wird. Im Vergleich zeigte sich, daß der so beschriebene Energieverlust weniger effektiv als in der Zwei-Teilchen-Beschreibung ist. Die Maximaltemperatur der Elektronen liegt somit - je nach gewählten Parametern - deutlich höher, und die Schicht ist insgesamt weiter ausgedehnt. Der Strahlungstransport durch diese unterschiedlich strukturierten Regionen liefert Spektren, die im gesamten Spektralbereich zum Teil erheblich voneinander abweichen, was den Anwendungsbereich der Näherungslösung stark einschränkt.

Aus Beobachtungen vieler magnetischer kataklysmischer Variable kann geschlossen werden, daß die Akkretionsregion eine endliche Ausdehnung auf der Sternoberfläche aufweist. Um den Einfluß der Geometrie auf die Schichtung und die resultierenden Spektren abzuschätzen, wurde ein einfaches Modell einer begrenzten Plasmasäule aufgestellt, in der die vertikale Temperatur- und Dichtestruktur herrscht, aber die Effekte des begrenzten Randes der Säule für den Strahlungstransport berücksichtigt werden. Es konnte gezeigt werden, daß diese Randeffekte für die Spektren der geheizten Region wichtig sind. Da somit aber ebenfalls die Strahlungskühlung des Fluids beeinflußt wird, ist für eine genauere Untersuchung ein noch zu erstellendes detaillierteres, zweidimensionales Modell der Akkretionsregion erforderlich.

Um die Ergebnisse des aufgestellten Modells für die Emissionsregion an einem konkreten System anzuwenden, wurden Beobachtungsdaten des Polars AM Herculis zu zwei Zuständen unterschiedlich hoher Akkretionstätigkeit verwendet. Zum einen wurde die Variabilität der optischen Strahlung während eines Bahnumlaufs des Doppelsterns in einem Zustand hoher Akkretionstätigkeit betrachtet und mit der Zyklotronstrahlung einer Akkretionssäule erklärt, die über die Oberfläche des weißen Zwergs hinausragt. Dabei konnte der Verlauf dieser "Lichtkurve" mit der Strahlung nur einer Emissionsregion erklärt werden, während bisherige Deutungen von einer Gewichtung mindestens zweier Akkretionsregionen (nahe den zwei Polen) ausgingen. 
Des weiteren wurde das Gesamtspektrum des Polars - vom infraroten Spektralbereich bis zur Röntgenstrahlung - zu einem Zustand niedriger und zu einem mit hoher Akkretion betrachtet. Es konnte gezeigt werden, daß das Gesamtspektrum in beiden Zuständen sowohl kleine wie auch große Massenstromdichten erfordert, da das jeweilige beobachtete Röntgenspektrum auch bei hohen Frequenzen noch Fluß aufweist. Dabei erstreckt sich die Verteilung der Massenstromdichten in der Akkretionsregion über sechs Dekaden. Der Zustand der hohen Akkretion unterscheidet sich in diesem Bild nur durch einen stärkeren Anteil der großen Massenstromdichten von dem einer geringen Akkretionstätigkeit. Aus dem aufgestellten Modell ergibt sich als natürliche Folge des Vorhandenseins hoher Massenstromdichten eine reprozessierte, schwarzkörperartige weiche Röntgenkomponente, wie sie auch in Beobachtungen gefunden wird. Die Anpassung der Beobachtungsdaten für den Zustand hoher Akkretion lieferte quantitativ den erforderlichen weichen Röntgenfluß.

Damit wurde erstmalig eine Verteilung von Massentromdichten in einer Akkretionsregion eines Polars abgeleitet, die auf den physikalischen Verhältnissen der Emissionsregion beruht und nicht eine ad hoc angenommene Gewichtung einzelner Beiträge zum Gesamtspektrum benutzt. Mit diesen Ergebnissen wird somit ein vertiefter Einblick in die Massentransferprozesse in Polaren geliefert, der ein quantitatives Verständnis der Entstehung des beobachteten Gesamtspektrums erlaubt.

Eine Erweiterung des Modells müßte in einer Beschreibung der Akkretionsregion in einer zweidimensionalen, kontinuierlichen Verteilung der Massenstromdichten bestehen, die auch eine explizite Zeitabhängigkeit der Akkretionsrate mit einbeziehen sollte. Da das zur Berechnung dieser Strukturen notwendige Differentialgleichungssystem (dreidimensionale, zeitabhängige Hydrodynamik und dreidimensionaler, zeitabhängiger Strahlungstransport) aber auch mit großem numerischem Aufwand vermutlich nur schwer zu lösen ist, kann eine Erweiterung des vorgestellten Modells wiederum nur zur Klärung eines Teilaspekts führen. 


\section{Literaturverzeichnis}

Aizu, K., 1973, X-Ray Emission Region of a White Dwarf with Accretion, Progr. Theor. Phys. 49, 1184

Bailey, J., Hough, J.H., Wickramasinghe, D.T., 1988, Photometry and Polarimetry of AM Herculis in its Faint State, MNRAS 233, 395

Bailey, J., Ferrario, L., Wickramasinghe, D.T., 1991, Cyclotron Features in the Infrared Spectrum of AM Herculis, MNRAS 251, 37p

Bekefi, G., 1966, Radiation Processes in Plasmas (New York: Wiley)

Beuermann, K., 1998, Magnetic Cataclysmic Variables: Observational and Theoretical Results, in: High Energy Astronomy \& Astrophysics, eds. P.C. Agrawal \& P.R. Vishwanath (Hyderabad: Universities Press (India) Limited), S. 100

Beuermann, K., Baraffe, I., Kolb, U., Weichhold, M., 1998, Are the Red Dwarfs in Cataclysmic Variables Main-Sequence Stars?, A\&A 339, 518

Beuermann, K., Burwitz, V., 1995, AM Herculis Binaries in the ROSAT Era, in: Cape Workshop on Magnetic Cataclysmic Variables, ASP Conference Series Vol. 85, eds. D.A.H. Buckley \& B. Warner (San Francisco: ASP), S. 99

Beuermann, K., Schwope, A.D., 1994, AM Herculis Binaries, in: Interacting Binary Stars, ASP Conference Series Vol. 56, ed. A.W. Shafter (San Francisco: ASP), S. 119

Beuermann, K., Stella, L., Patterson, J., 1987, EINSTEIN Observations of EF Eridani (2A 0311-227), The Textbook Example of AM Herculis-Type Systems, ApJ 316, 360

Braginskii, S.I., 1958, Transport Phenomena in a Completely Ionized Two-Temperature Plasma, Soviet Phys. JETP 6, 358

Burleigh, M.R., Jordan, S., Schweizer, W., 1999, Phase-Resolved Far-Ultraviolet Hubble Space Telescope Spectroscopy of the Peculiar Magnetic White Dwarf RE J0317-853, ApJ 510, L37

Chanmugam, G., Barrett, P.E., Wu, K., Courtney, M.W., 1989, Thermal Cyclotron Absorption Coefficients, ApJSupplSer 71, 323

Chanmugam, G., Dulk, G.A., 1981, Polarized Radiation from Hot Plasmas and Applications to AM Herculis Binaries, ApJ 244, 569

Chanmugam, G., Langer, S.H., 1991, Formulae for the Cyclotron Luminosity of Magnetized Plasma Slabs, ApJ 368, 580

Chanmugam, G., Langer, S.H., Shaviv, G., 1985, Time-Dependent Accretion onto Magnetic White Dwarfs: Effects of Cyclotron Emission, ApJ 299, L87 
Chanmugam, G., Wagner, R.L., 1979, Cyclotron Self-Absorption in Accretion Columns of Magnetic Degenerate Stars, ApJ 232, 895

Chevalier, R.A., Imamura, J.N., 1982, Linear Analysis of an Oscillatory Instability of Radiative Shock Waves, ApJ 261, 543

Cropper, M., 1990, The Polars, Space Sci. Rev. 54, 195

Cropper, M., Menzies, J.W., Tapia, S., 1986, E1405-451: Three Seasons of Polarimetry and Photometry, MNRAS 218, 201

Eggleton, P.P., 1976, Angular Momentum Loss and the Origin of Cataclysmic Binaries, in: Structure and Evolution of Close Binary Systems. IAU Symposium No. 73, eds. P.P. Eggleton, S. Mitton \& J. Whelan (Dordrecht: Reidel), S. 209

Fabian, A.C., Pringle, J.E., Rees, M.J., 1976, X-Ray Emission from Accretion on to White Dwarfs, MNRAS 175, 43

Frank, J., King, A.R., 1984, Column Accretion onto White Dwarfs: Non-Local Electron Transport and the Soft X-Ray Excess, A\&A 134, 328

Frank, J., King, A.R., Lasota, J.-P., 1983, Column Accretion on to White Dwarfs, MNRAS 202,183

Frank, J., King, A.R., Lasota, J.-P., 1988, The Soft X-Ray Excess in Accreting Magnetic White Dwarfs, A\&A 193, 113

Frank, J., King, A.R., Raine, D., 1992, Accretion Power in Astrophysics (2nd ed.; Cambridge: University Press)

Gänsicke, B.T., 1997, Heating and Cooling of Accreting White Dwarfs, Dissertation, Georg-August-Universität Göttingen

Gänsicke, B.T., 1999, private Mitteilung

Gänsicke, B.T., Beuermann, K., de Martino, D., 1995, The White Dwarf in AM Herculis, A\&A 303, 127

Gänsicke, B.T., Hoard, D.W., Beuermann, K., Sion, E.M., Szkody, P., 1998, HST/GHRS Observations of AM Herculis, A\&A 338, 933

Hameury, J.M., King, A.R., 1988, The X-Ray Light Curves of AM Herculis Systems, MNRAS 235, 433

Hameury, J.M., King, A.R., Lasota, J.-P., 1986, Accretion Flows in the Non-Synchronous Magnetic Cataclysmic Variables, MNRAS 218, 695

Heerlein, C., Horne, K., Schwope, A.D., 1999, Modelling of the Magnetic Accretion Flow in HU Aquarii, MNRAS 304, 145

Hoshi, R., 1973, X-Ray Emission from White Dwarfs in Close Binary Systems, Progr. Theor. Phys. 49, 776

Imamura, J.N., Aboasha, A., Wolff, M.T., Wood, K.S., 1996, The Stability Properties of Two-Temperature White Dwarf Radiative Shock Waves, ApJ 458, 327 
Imamura, J.N., Durisen, R.H., Lamb, D.Q., Weast, G.J., 1987, X-Ray and Ultraviolet Radiation from Accreting White Dwarfs. IV. Two-Temperature Treatment with Electron Thermal Conduction, ApJ 313, 298

Imamura, J.N., Wolff, M.T., Durisen, R.H., 1984, A Numerical Study of the Stability of Radiative Shocks, ApJ 276, 667

Ishida, M., Matsuzaki, K., Fujimoto, R., Mukai, K., Osborne, J.P., 1997, Detailed X-Ray Spectroscopy of AM Herculis with ASCA, MNRAS 287, 651

Johnson, H.L., 1965, Interstellar Extinction in the Galaxy, ApJ 141, 923

King, A.R., Lasota, J.-P., 1980, Accretion on to Highly Magnetized White Dwarfs - The Role of Electron Conduction, MNRAS 191, 721

Kippenhahn, R., Weigert, A., 1990, Stellar Structure and Evolution (Berlin: Springer)

Krolik, J.H, Kallman, T.R., 1984, Soft X-Ray Opacity in Hot and Photoionized Gases, ApJ 286, 366

Kuijpers, J., Pringle, J.E., 1982, Comments on Radial White Dwarf Accretion, A\&A 114, L4

Kylafis, N.D., Lamb, D.Q., 1979, X-Ray and UV Radiation from Accreting Nonmagnetic Degenerate Dwarfs, ApJ 228, L105

Kylafis, N.D., Lamb, D.Q., 1982, X-Ray and UV Radiation from Accreting Nonmagnetic Degenerate Dwarfs. II., ApJSupplSer 48, 239

Lamb, D.Q., 1985, Recent Developments in the Theory of AM Her and DQ Her Stars, in: Cataclysmic Variables and Low-Mass X-ray Binaries; Proceedings of the Seventh North American Workshop, eds. D.Q. Lamb \& J. Patterson (Dordrecht: Reidel), S. 179

Lamb, D.Q., Masters, A.R., 1979, X and UV Radiation from Accreting Magnetic Degenerate Dwarfs, ApJ 234, L117

Landau, L.D., Lifshitz, E.M., 1962, The Classical Theory of Fields (2nd ed.; Oxford: Pergamon Press)

Landau, L.D., Lifshitz, E.M., 1963, Fluid Mechanics (2nd ed.; Oxford: Pergamon Press)

Langer, S.H., Chanmugam, G., Shaviv, G., 1981, Thermal Instability in Accretion Flows onto Degenerate Stars, ApJ 245, L23

Langer, S.H., Chanmugam, G., Shaviv, G., 1982, Time-Dependent Accretion onto Magnetized White Dwarfs, ApJ 258, 289

Larsson, S., 1987, Discovery of 2-3 s Quasi-Periodic Oscillations in EF Eri, A\&A 181, L15

Larsson, S., 1988, Discovery of 1 Second Optical QPO in VVPuppis, Advances in Space Research 8, 305

Larsson, S., 1989, Optical One Second Quasi-Periodic Oscillations in VVPuppis, A\&A 217,146

Larsson, S., 1992, Rapid Optical Variability in the AM Herculis Object V 834 Centauri. I. Quasi-Periodic Oscillations, A\&A 265, 133 
Leggett, S.K., Allard, F., Berriman, G., Dahn, C.C., Hauschildt, P.H., 1996, Infrared Spectra of Low-Mass Stars: Toward a Temperature Scale for Red Dwarfs, ApJSupplSer 104,117

Levich, E.V., Sunyaev, R.A., 1971, Heating of Gas near Quasars, Seyfert-Galaxy Nuclei, and Pulsars by Low-Frequency Radiation, Soviet Astronomy - AJ 15, 363

Liebert, J., Stockman, H.S., 1985, The AM Herculis Magnetic Variables, in: Cataclysmic Variables and Low-Mass X-ray Binaries; Proceedings of the Seventh North American Workshop, eds. D.Q. Lamb \& J. Patterson (Dordrecht: Reidel), S. 151

Litchfield, S.J., King, A.R., 1990, Blob Accretion in AM Herculis Systems, MNRAS 247, 200

Livio, M., 1994, Topics in the Theory of Cataclysmic Variables and X-Ray Binaries, in: Interacting Binaries. 22nd Saas-Fee Advanced Course, eds. H. Nussbaumer \& A. Orr (Berlin: Springer), S. 135

Lubow, S.H., Shu, F.H., 1975, Gas Dynamics of Semidetached Binaries, ApJ 198, 383

Lubow, S.H., Shu, F.H., 1976, Gas Dynamics of Semidetached Binaries. II - The Vertical Structure of the Stream, ApJ 207, L53

Masters, A.R., Fabian, A.C., Pringle, J.E., Rees, M.J., 1976, Cyclotron Emission from Accreting Magnetic White Dwarfs, MNRAS 178, 501

Middleditch, J., 1982, Two-Second Variability in AM Herculis Binaries, ApJ 257, L71

Middleditch, J., Imamura, J.N., Steiman-Cameron, T.Y., 1997, Discovery of QuasiPeriodic Oscillations in the AM Herculis Object BL Hydri, ApJ 489, 912

Mihalas, D., 1978, Stellar Atmospheres (2nd ed.; San Francisco: Freeman)

Nauenberg, M., 1972, Analytic Approximations to the Mass-Radius Relation and Energy of Zero-Temperature Stars, ApJ 175, 417

Panek, R.J., 1980, On the Rapid Variability of AM Herculis, ApJ 241, 1077

Priedhorsky, W., Matthews, K., Neugebauer, G., Werner, M., Krzeminski, W., 1978, Joint Infrared and Visual Monitoring of AM Herculis, ApJ 226, 397

Raith, K., Schönauer, W., Glotz, G., 1979, SLGA - Ein selbststeuerndes Lösungsverfahren für Anfangswertprobleme bei gewöhnlichen Differentialgleichungen, Interner Bericht 13/79 des Rechenzentrums der Universität Karlsruhe

Ramaty, R., 1969, Gyrosynchrotron Emission and Absorption in a Magnetoactive Plasma, ApJ 158, 753

Ramsay, G., Mason, K.O., Cropper, M., Watson, M.G., Clayton, K.L., 1994, ROSAT Observations of AN UMa and MR Ser: The Status of the Soft X-Ray Excess in AM Her Stars, MNRAS 270, 692

Raymond, J.C., Black, J.H., Davis, R.J., Dupree, A.K., Gursky, H., Hartmann, L., Matilsky, T.A., 1979, Ultraviolet Observations of AM Herculis with IUE, ApJ 230, L95

Rybicki, G.B., Lightman, A.P., 1979, Radiative Processes in Astrophysics (New York: Wiley-Interscience Publication) 
Saxton, C.J., Wu, K., Pongracic, H., 1997, Stability of Accretion Shocks with a Composite Cooling Function, Proc. Astron. Soc. Australia 14, 164

Saxton, C.J., Wu, K., Pongracic, H., Shaviv, G., 1998, Oscillatory Instability of Radiative Shocks with Multiple Cooling Processes, MNRAS 299, 862

Southwell, K.A., Still, M.D., Smith, R.C., Martin, J.S., 1995, The Mass Ratio of the Cataclysmic Variable AM Herculis, A\&A 302, 90

Spitzer, L., 1962, Physics of Fully Ionized Gases (2nd ed.; New York: Interscience Publishers)

Steiman-Cameron, T.Y., Young, K., Scargle, J.D., Crutchfield, J.P., Imamura, J.N., Wolff, M.T., Wood, K.S., 1994, Dripping Handrails and the Quasi-Periodic Oscillations of the AM Herculis Objects, ApJ 435, 775

Stockman, H.S., 1988, Accretion Shock Geometries in the Magnetic Variables, in: Polarized Radiation of Circumstellar Origin, eds. G.V. Coyne (S.J.), A.M. Magalhães, A.F.J. Moffat, R.E. Schulte-Ladbeck, S. Tapia \& D.T. Wickramasinghe (Tucson: University of Arizona Press), S. 237

Szkody, P., Brownlee, D.E., 1977, AM Herculis: A Unique X-Ray Binary as Revealed Through the Optical Light Curve, ApJ 212, L113

Thompson, A.M., Brown, J.C., Kuijpers, J., 1986, Bombardment Solutions to the "Soft X-Ray Puzzle" in Radial White Dwarf Accretion, A\&A 159, 202

Thompson, A.M., Cawthorne, T.V., 1987, Cyclotron Emission from White Dwarf Accretion Columns, MNRAS 224, 425

van Teeseling, A., Kaastra, J.S., Heise, J., 1996, Iron K Fluorescence and Compton Reflection in Magnetic Cataclysmic Variables, A\&A 312, 186

Wada, T., Shimizu, A., Suzuki, M., Kato, M., Hoshi, R., 1980, X-Ray and Infrared Emissions from Accreting Magnetic White Dwarfs, Progr. Theor. Phys. 64, 1986

Warner, B., 1995, Cataclysmic Variable Stars (Cambridge: University Press)

Weymann, R., 1965, Diffusion Approximation for a Photon Gas Interacting with a Plasma via the Compton Effect, Phys. Fluids 8, 2112

Wickramasinghe, D.T., Bailey, J., Meggitt, S.M.A., Ferrario, L., Hough, J., Tuohy, I.R., 1991, New Polarimetric Observations and a Two Pole Model for the Cyclotron Emission from AM Herculis, MNRAS 251, 28

Williams, G.A., King, A.R., Brooker, J.R.E., 1987, LTE Model Atmospheres for Accreting White Dwarfs, MNRAS 226, 725

Woelk, U., 1991, Zyklotronstrahlung in teilchengeheizten Atmosphären magnetischer WeiBer Zwerge, Dissertation, Technische Universität Berlin

Woelk, U., Beuermann, K., 1992, Particle Heated Atmospheres of Magnetic White Dwarfs, A\&A 256, 498

Woelk, U., Beuermann, K., 1993, Temperature Structure of a Particle-Heated Magnetic Atmosphere, A\&A 280, 169

Woelk, U., Beuermann, K., 1996, Stationary Radiation Hydrodynamics of Accreting Magnetic White Dwarfs, A\&A 306, 232 
Wolff, M.T., Gardner, J.H., Wood, K.S., 1989, On the Stability of Degenerate White Dwarf Radiative Shocks: New One- and Two-Temperature Calculations, ApJ 346, 833

Wolff, M.T., Wood, K.S., Imamura, J.N., 1991, Noise-Driven Radiative Shocks: A New Model for the Optical Quasi-Periodic Oscillations of the AM Herculis Objects, ApJ 375, L31

Wood, K.S., Imamura, J.N., Wolff, M.T., 1992, Noise-Driven Radiative Shocks. II. Further Implications for the Quasi-Periodic Oszillations of the AM Herculis Objects, ApJ 398, 593

Wu, K., 1994, Structure of Inhomogeneous Accretion Shocks, Proc. Astron. Soc. Australia 11,61

Wu, K., Chanmugam, G., Shaviv, G., 1992, Properties of Quasi-Periodic Oscillations in Accreting White Dwarfs, ApJ 397, 232

Wu, K., Chanmugam, G., Shaviv, G., 1994, Structure of Steady State Accretion Shocks with Several Cooling Functions: Closed Integral-Form Solution, ApJ 426, 664

Wu, K., Pongracic, H., Chanmugam, G., Shaviv, G., 1996, Simulations of Quasi-Periodic Oscillations in Accreting White Dwarfs, Proc. Astron. Soc. Australia 13, 93

Wu, K., Saxton, C.J., 1999, Stability Properties of Two-Fluid Accretion Shocks with Multiple Cooling Processes, in: Annapolis Workshop on Magnetic Cataclysmic Variables, ASP Conference Series Vol. 157, eds. C. Hellier \& K. Mukai (San Francisco: ASP), S. 317

Zel'dovich, Ya.B., Raizer, Yu.P., 1966, Physics of Shock Waves and High-Temperature Hydrodynamic Phenomena (New York: Academic Press) 


\section{Danksagung}

Vielen Menschen bin ich zu Dank verpflichtet.

Zuerst möchte ich Prof. Dr. Klaus Beuermann danken, der mich bei der Anfertigung dieser Arbeit betreute und mir immer mit Rat und Tat zur Verfügung stand. Von seiner Erfahrung in astrophysikalischen Belangen und seinem Reichtum an Ideen habe ich oft profitiert. Er zwang mich immer wieder, mich auf die wesentlichen Fragestellungen zu beschränken.

Besonderen Dank möchte ich auch Dr. Boris T. Gänsicke aussprechen, der mir in Fragen bezüglich der Beobachtungsergebnisse von Polaren weiterhalf und mich immer wieder motivierte, die Arbeit fortzusetzen.

Dr. Ulrich Woelk bin ich zu großem Dank verpflichtet, da ohne seine Vorleistungen die Abfassung dieser Arbeit sehr viel mehr Zeit in Anspruch genommen hätte.

Weiterhin danke ich allen Mitarbeitern der Universitäts-Sternwarte Göttingen für das anregende Arbeitsklima. Besonders erwähnen möchte ich: Dr. Vadim Burwitz, Stephan Eisenbart, Dr. Patrick Frisch, Ejdar Gözlü, Dr. Frederic Hessman, Christian Hettlage, Dr. Elmar Kemnade, Dr. Ralf Kleiber, Jens Kube, Dr. Stefanie Mehren, Dr. Carsten Obach, Dr. Klaus Reinsch, Frank Rieger, Thierry Rousseau, Matthias Schreiber, Marcus Schulte und Dr. André van Teeseling, die auch außerhalb des Themenkreises der Astrophysik interessante Diskussionspartner waren.

Vielen Dank an Dr. Roberto Silvotti (Osservatorio di Capodimonte, Neapel) für die Erlaubnis, die AM Her Lichtkurve vor einer Veröffentlichung zu verwenden.

Das Korrekturlesen dieser Arbeit besorgten Jens Kube und Dr. Boris T. Gänsicke. Ihren geleisteten Dienst weiß ich zu schätzen.

Für die finanzielle Unterstützung in der Endphase der Arbeit, habe ich der Deutschen Forschungsgemeinschaft als Trägerin des Graduiertenkollegs „Strömungsinstabilitäten und Turbulenz" zu danken.

Allen meinen Freunden danke ich, daß sie über viele Jahre hinweg, trotz meines Eigensinns, nicht müde wurden mir Wege aufzuzeigen. Ich hoffe auch in Zukunft auf sie zählen zu können.

Meine Familie brachte mir immer Hilfe entgegen und hatte stets Verständnis für meine Belange. Ihre Mühen und Entbehrungen sind durch bloßes bedanken nicht zu vergelten. Ihnen schulde ich viel mehr als sich in einer „Danksagung“ beschreiben läßt. 



\section{Veröffentlichungen}

\section{Referierte Zeitschriften}

Rousseau, T., Fischer, A., Beuermann, K., Woelk, U., 1996, Determination of mass flow rates in AM Herculis binaries: General method and application to UZ For, A\&A 310, 526

\section{Konferenzbeiträge}

Fischer, A., Rousseau, T., Beuermann, K., Woelk, U., 1996, Radiation hydrodynamics and soft X-ray emission of magnetic white dwarfs, Astron. Ges. Abstract Series 12, 187

Beuermann, K., Fischer, A., Rousseau, T., Woelk, U., 1997, Radiation hydrodynamics and soft X-ray emission of magnetic white dwarfs, in: Proceedings IAU Colloquium 163, "Accretion Phenomena and Related Outflows", ASP Conference Series Vol. 121, eds. Wickramasinghe, D., Ferrario, L., Bicknell, G. (San Francisco: ASP), S. 687

Fischer, A., Beuermann, K., Woelk, U., 1998, The structure of the accretion column in AM Her stars and implications on mass determination of the white dwarf, Astron. Ges. Abstract Series 14, 29

van Teeseling, A., Fischer, A., Beuermann, K., 1999, X-ray emission from the post-shock flow, in: Annapolis Workshop on Magnetic Cataclysmic Variables, ASP Conference Series Vol. 157, eds. C. Hellier \& K. Mukai (San Francisco: ASP), S. 309 



\section{Lebenslauf}

\section{Daten}

Name

Geburtsdatum

Geburtsort

Staatsangehörigkeit

Familienstand

\section{Schulausbildung}

$1973-77$

$1977-79$

$1979-83$

$1983-86$
Andreas Fischer

23. August 1966

Northeim

deutsch

ledig

Martin-Luther Grundschule, Northeim

Orientierungsstufe der Thomas-Mann-Schule, Northeim

Realschule Northeim

Fachgymnasium Technik der Berufsbildenden Schulen II, Göttingen

Abschluß: Allgemeine Hochschulreife

Wehrdienst

$1986-87$

Grundwehrdienst bei PzGrenBtl 43, Göttingen

\section{Hochschulausbildung}

Oktober 1987

April 1990

Februar 1994

Seit 1996

Seit 1998

\section{Tätigkeiten}

$1992-98$

Sommersem. 1997
Aufnahme des Physikstudiums an der Georg-August-Universität zu Göttingen Vordiplom in Physik

Diplom in Physik

Thema der Diplomarbeit: „Polarisationseigenschaften der Zyklotronstrahlung von AM Herculis Sternen“, Betreuer: Prof. Dr. Klaus Beuermann, UniversitätsSternwarte, Göttingen

Anfertigung der vorliegenden Dissertation unter der Betreuung von Prof. Dr. Klaus Beuermann

Stipendiat des Graduiertenkollegs „Strömungsinstabilitäten und Turbulenz“

Wissenschaftliche Hilfskraft an der Universitäts-Sternwarte, Göttingen

Durchführung der Lehrveranstaltung „Praktikum Informatik“ an der Fachhochschule Hildesheim/Holzminden, Fachbereich Physik-, Meß- und Feinwerktechnik in Göttingen 
